The Effect of Blade Solidity on the Aerodynamic Loss

of a Transonic Turbine Cascade

by

Roger L. Doughty

thesis submitted to the Faculty of the

Virginia Polytechnic Institute and State University

in partial fulfillment of the requirements for the degree of

Master of Science

in

Mechanical Engineering

APPROVED:

chit t. Dancey

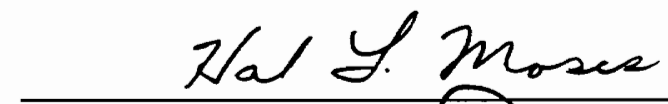

Dr. H. L. Moses, chairman

Dr. C. L. Danceyo

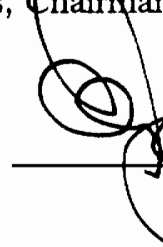

Dr. E. F. Brown

April 1991

Blacksburg, Virginia 
2.2

LD

5655

1855

1991

1684
$c .2$ 


\section{The Effect of Blade Solidity on the Aerodynamic Loss \\ of a Transonic Turbine Cascade \\ by}

Roger L. Doughty

Dr. H. L. Moses, Chairman

Mechanical Engineering

(ABSTRACT)

Past research at Virginia Tech (VPI) explored the aerodynamic loss of the transonic VPI turbine blade, which is based on the pitchline profile of a high pressure turbine blade for a large commercial aircraft gas turbine. The current experiment explores the loss of the VPI blade for different axial solidity ratios near the design point. Ten percent changes in the solidity ratio were accomplished by varying the blade pitch and changing the blade stagger to maintain a constant throat to spacing ratio. Reaction, exit angle and exit Mach number were kept constant with this method. Cascades with three different solidities were tested in VPI's transonic blow-down wind tunnel. Downstream total pressure loss and static pressure measurements were obtained. In addition, inviscid calculations were made for each case. Static pressure contours and Mach number profiles from the calculations were compared with the experimental results.

A ten percent decrease in solidity caused no cascade loss penalty as compared to the Baseline solidity for a wide range of Mach numbers. Calculated blade Mach number profiles agreed well with experimental profiles except on the suction side near the throat and downstream of the shock/boundary layer interaction. Predicted downstream static pressure values agreed well with experimental values, except that the inviscid code tended to over-predict the pressure rise across the suction side shocks. 


\section{Acknowledgements}

I am indebted to Dr. Hal Moses for his direction and patient advice during the course of this research. He often provided the insight necessary to get at the root of a problem when I was stuck.

This work would not have been possible without the financial and technical support of General Electric Aircraft Engines in Cincinnati, Ohio. Specifically, support has been provided through the efforts of Brent Gregory, manager of Turbine Aero and Cooling Technologies. At his invitation I spent two summer months in Cincinatti working on the computational portion of this research using GEAE's software. I appreciate that opportunity to learn about the design process for aircraft gas turbine engines. While at GEAE I met and worked with several other engineers who provided me with invaluable help and direction. In particular I would like to mention Scott Hunter, Dave Cherry, Monty Shelton, and Ann Isburgh. Thanks to all who made my stay in Cincinnati so enlightening.

My fellow graduate students provided the humor necessary to get through a year of experimental work. Thanks to Tibor Kiss, Remi Bertsch, and Jeff Collie for undying willingness to help in the windtunnel at odd hours and on a moments notice. I enjoyed the endless games of Tetris and electronic Golf with these guys while waiting for the compressor to pump up to operating pressure. Sadly I will never be in their skill level when it comes to computer games. 
Thanks finally to Dr. Clinton Dancey and Dr. Eugene Brown for serving on my graduate committee. I appreciate Dr. Dancey's willingness to help a fellow Comell alumnus learn the ropes at Virginia Tech.

To all the unsung heroes I forgot to mention, thanks a bunch. 


\section{Table of Contents}

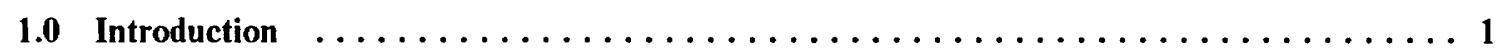

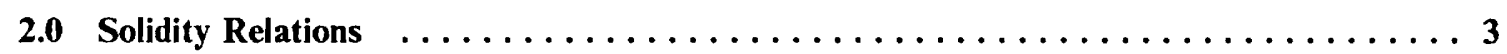

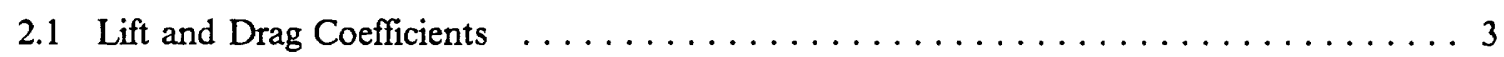

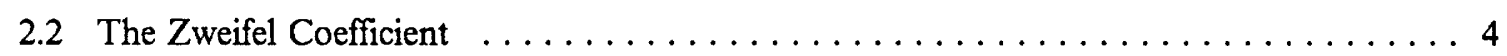

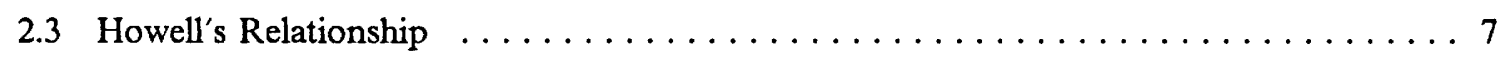

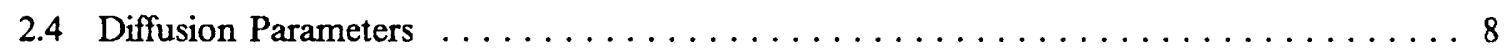

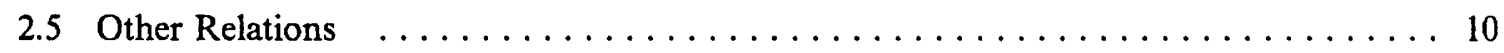

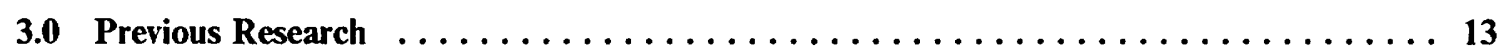

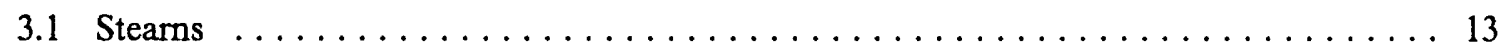

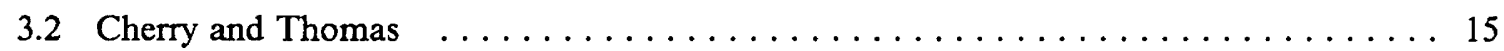

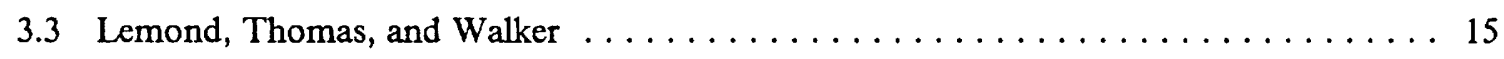

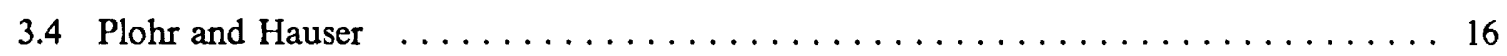

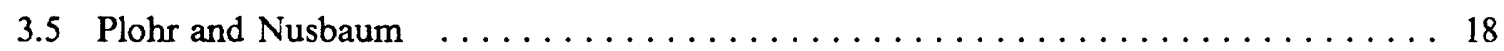

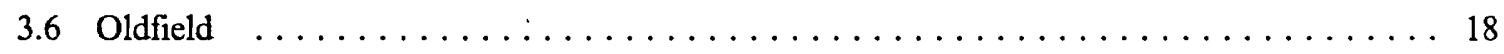




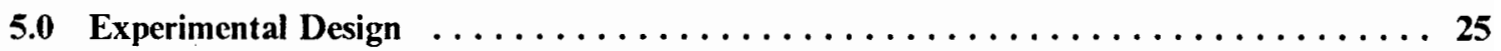

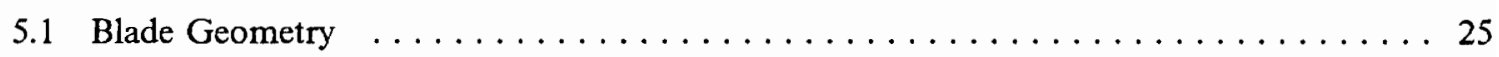

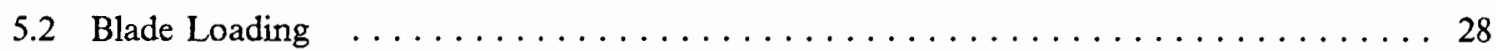

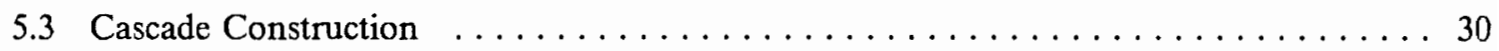

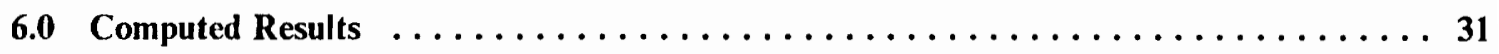

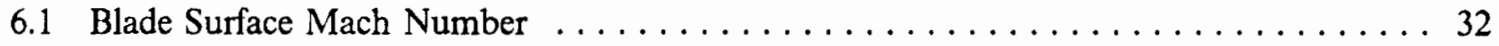

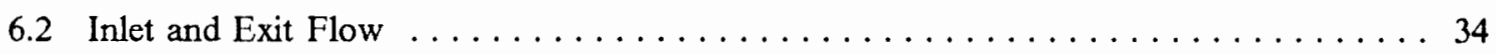

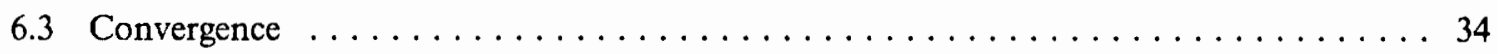

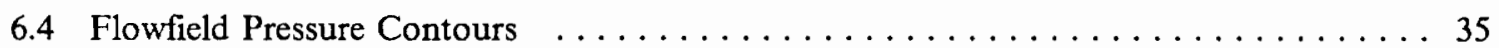

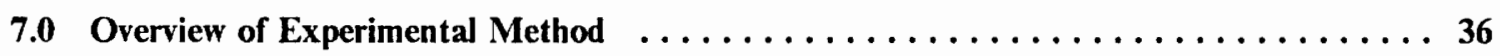

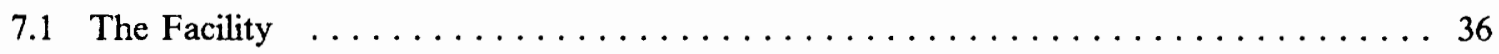

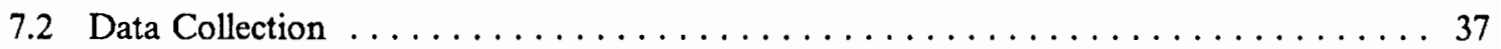

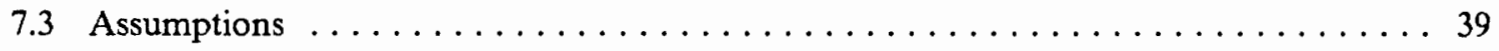

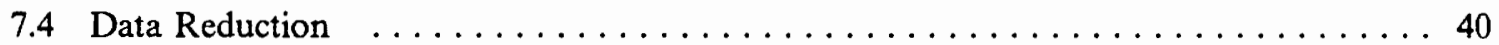

8.0 Experimental Results $\ldots \ldots \ldots \ldots \ldots \ldots \ldots \ldots \ldots \ldots \ldots \ldots \ldots \ldots \ldots \ldots$

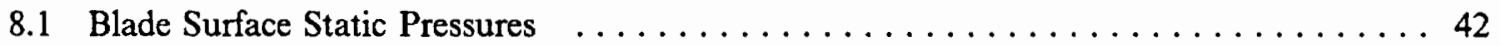

8.2 Comparison of Blade Loadings $\ldots \ldots \ldots \ldots \ldots \ldots \ldots \ldots \ldots \ldots \ldots \ldots \ldots$

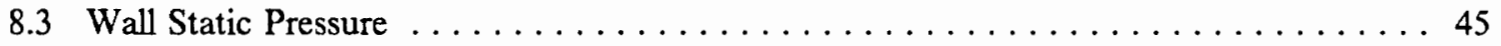

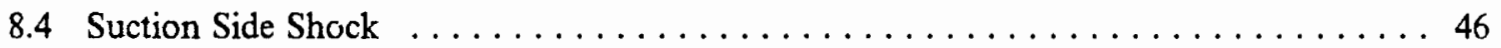

8.5 Base Pressure and Unguided Turning $\ldots \ldots \ldots \ldots \ldots \ldots \ldots \ldots \ldots \ldots \ldots$

8.6 Traverse Data . . . . . . . . . . . . . . . . . . . . . . . . 49

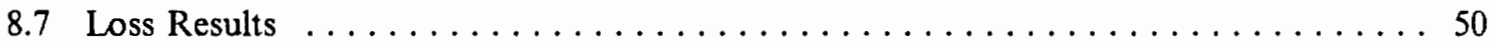

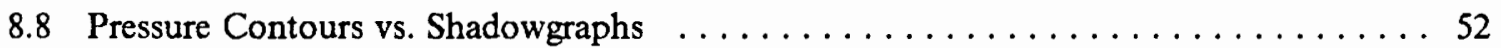




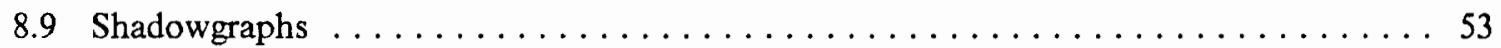

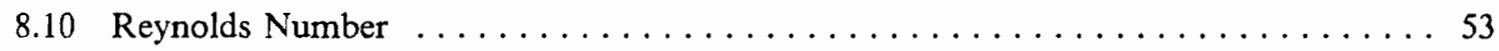

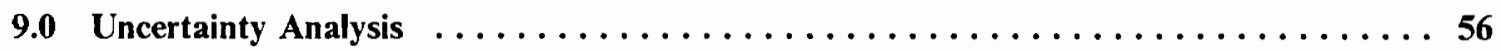

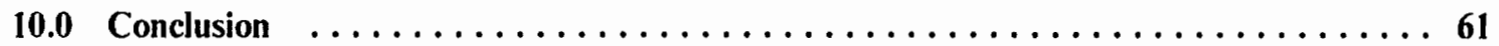

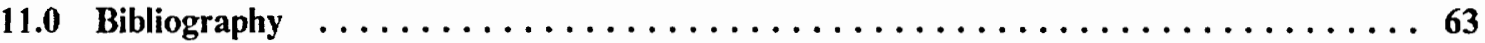

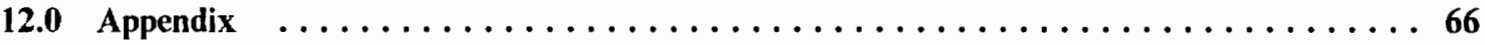

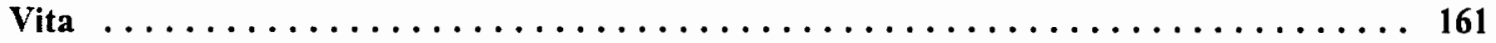




\section{List of Tables}

Table 1. Comparison of blade design parameters $\ldots \ldots \ldots \ldots \ldots \ldots \ldots \ldots \ldots$

Table 2. Computational input parameters and results $\ldots \ldots \ldots \ldots \ldots \ldots \ldots$

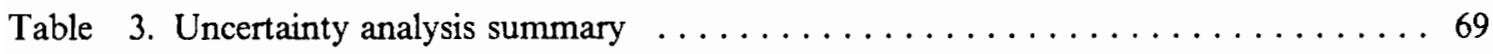




\section{List of Illustrations}

Figure 1. Conventions used for Zweifel calculations $\ldots \ldots \ldots \ldots \ldots \ldots \ldots \ldots$

Figure 2. Zweifel's notation for turbine pressure distributions $\ldots \ldots \ldots \ldots \ldots \ldots \ldots$

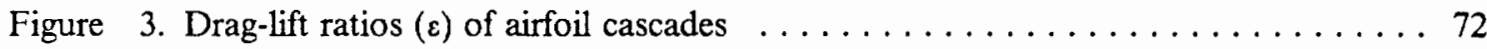

Figure 4. Relationship between Howell's Lift and Drag coefficients $\ldots \ldots \ldots \ldots \ldots$

Figure 5. Axial solidity parameter vs. reaction and diffusion $\ldots \ldots \ldots \ldots \ldots \ldots$

Figure 6. Relative orientation of blades for solidity tests $\ldots \ldots \ldots \ldots \ldots \ldots$

Figure 7 . Relative spacing of blades for solidity tests $\ldots \ldots \ldots \ldots \ldots \ldots \ldots$

Figure 8. Physical comparison of original blade and VPI blade $\ldots \ldots \ldots \ldots \ldots \ldots 77$

Figure 9. Mach number comparison of original blade and VPI blade $\ldots \ldots \ldots \ldots \ldots 78$

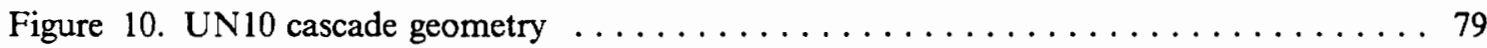

Figure 11 . Baseline cascade geometry $\ldots \ldots \ldots \ldots \ldots \ldots \ldots \ldots \ldots \ldots \ldots \ldots$

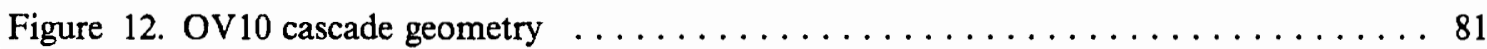

Figure 13. Endplate outer dimensions $\ldots \ldots \ldots \ldots \ldots \ldots \ldots \ldots \ldots \ldots \ldots \ldots \ldots \ldots \ldots \ldots \ldots \ldots$

Figure 14. Predicted blade surface Mach number distributions $\ldots \ldots \ldots \ldots \ldots \ldots \ldots$

Figure 15. Predicted blade surface static pressure distributions $\ldots \ldots \ldots \ldots \ldots$

Figure 16. Predicted pressure contours, UN10 cascade, $P_{t 1} / P_{2}=2.77 \ldots \ldots \ldots \ldots$

Figure 17. Predicted pressure contours, Baseline cascade, $P_{t 1} / P_{2}=2.787 \ldots \ldots \ldots$

Figure 18. Predicted pressure contours, OV10 cascade, $P_{t 1} / P_{2}=2.71 \ldots \ldots \ldots \ldots$

Figure 19. Transonic windtunnel test section $\ldots \ldots \ldots \ldots \ldots \ldots \ldots \ldots \ldots \ldots$

Figure $20 . \mathrm{UN} 10$ cascade $\ldots \ldots \ldots \ldots \ldots \ldots \ldots \ldots \ldots \ldots \ldots \ldots$

Figure 21. Isentropic Mach number versus upstream total pressure correlation $\ldots \ldots \ldots 90$ 
Figure 22. Rear static pressure versus upstream total pressure correlation $\ldots \ldots \ldots \ldots 9$

Figure 23. Blade surface static pressure distribution: $-10 \%$ solidity, $\mathrm{P}_{t 1} / \mathrm{P}_{2}=2.77 \ldots \ldots 2$

Figure 24. Blade surface static pressure distribution: Baseline solidity, $\mathrm{P}_{t 1} / \mathrm{P}_{2}=2.78 \ldots 93$

Figure 25. Blade surface static pressure distribution: $+10 \%$ solidity, $\mathrm{P}_{t 1} / \mathrm{P}_{2}=2.71 \ldots 94$

Figure 26. Blade surface Mach number distributions: Three solidities, $M_{2 i s} \simeq 0.82 \ldots 95$

Figure 27. Blade surface Mach number distributions: Three solidities, $M_{2 i s} \simeq 0.97 \ldots 96$

Figure 28. Blade surface Mach number distributions: Three solidities, $M_{2 i s} \simeq 1.03 \ldots 97$

Figure 29. Blade surface Mach number distributions: Three solidities, $M_{2 i s} \simeq 1.11 \ldots 98$

Figure 30. Blade surface Mach number distributions: Three solidities, $M_{2 i s} \simeq 1.19 \ldots 99$

Figure 31. Blade surface Mach number distributions: Three solidities, $M_{2 i s} \simeq 1.24 \ldots 100$

Figure 32. Blade surface Mach number distributions: Three solidities, $M_{2 i s} \simeq 1.35 \ldots 101$

Figure 33. Blade surface static pressure distributions: UN10 cascade, $\sigma=0.923 \ldots \ldots 2$

Figure 34. Blade surface static pressure distributions: Baseline cascade, $\sigma=1.025 \ldots \ldots 103$

Figure 35. Blade surface static pressure distributions: OV10 cascade, $\sigma=1.128 \ldots \ldots 104$

Figure 36. Blade surface Mach number distributions: UN10 cascade, $\sigma=0.923 \ldots \ldots 105$

Figure 37. Blade surface Mach number distributions: Baseline cascade, $\sigma=1.025 \ldots \ldots 6$

Figure 38. Blade surface Mach number distributions: OV10 cascade, $\sigma=1.128 \ldots \ldots 107$

Figure 39. Location of blade static pressure taps $\ldots \ldots \ldots \ldots \ldots \ldots \ldots \ldots \ldots$

Figure 40. Wall static pressure, UN10 cascade, Station $1 \ldots \ldots \ldots \ldots \ldots \ldots$

Figure 41 . Wall static pressure, UN10 cascade, Station $2 \ldots \ldots \ldots \ldots \ldots \ldots$

Figure 42. Wall static pressure, Baseline cascade, Station $1 \ldots \ldots \ldots \ldots \ldots \ldots \ldots$

Figure 43 . Wall static pressure, Baseline cascade, Station $2 \ldots \ldots \ldots \ldots \ldots \ldots \ldots$

Figure 44 . Wall static pressure, OV10 cascade, Station $1 \ldots \ldots \ldots \ldots \ldots \ldots \ldots$

Figure 45. Wall static pressure, OV10 cascade, Station $2 \ldots \ldots \ldots \ldots \ldots \ldots \ldots \ldots$

Figure 46. Static pressure rise across suction side shock, Station $1 \ldots \ldots \ldots \ldots \ldots$

Figure 47. Static pressure rise across suction side shock, Station $2 \ldots \ldots \ldots \ldots \ldots$

Figure 48. Suction side shock angle versus isentropic Mach number $\ldots \ldots \ldots \ldots \ldots 117$

Figure 49. Blade base pressure ratio versus downstream pressure ratio $\ldots \ldots \ldots \ldots \ldots$ 
Figure 50. Comparison of experimental and predicted blade base pressures, $\mathrm{M}_{2 i s}=1.30 \quad \ldots \quad 119$

Figure 51. Sieverding's base pressure correlation $\ldots \ldots \ldots \ldots \ldots \ldots \ldots \ldots \ldots \ldots$

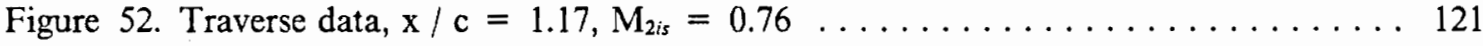

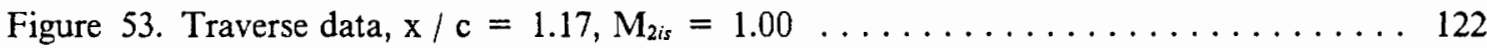

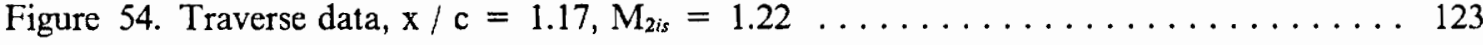

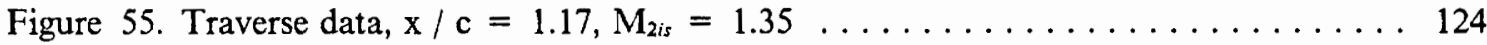

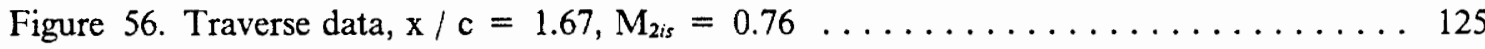

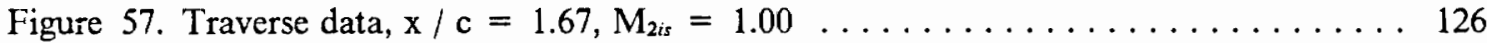

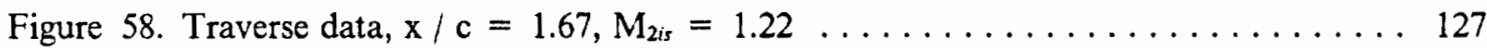

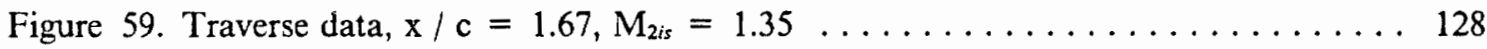

Figure 60. Corrected total pressure loss versus isentropic Mach number, all cascades, Station

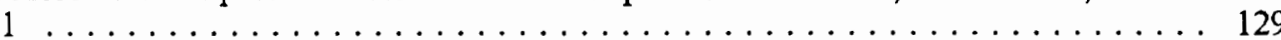

Figure 61. Corrected total pressure loss versus isentropic Mach number, all cascades, Station

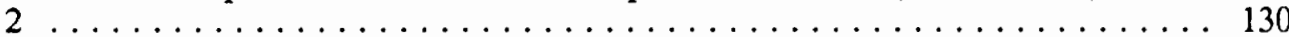

Figure 62. Corrected wake total pressure loss, all cascades, Station $1 \ldots \ldots \ldots \ldots \ldots 131$

Figure 63. Uncorrected total pressure loss versus isentropic Mach number, all cascades,

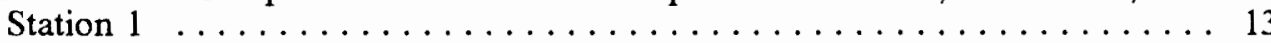

Figure 64. Uncorrected total pressure loss versus isentropic Mach number, all cascades, Station 2

Figure 65. Corrected total pressure loss versus isentropic Mach number, UN10 cascade . . 134

Figure 66. Corrected total pressure loss versus isentropic Mach number, Baseline cascade . 135

Figure 67. Corrected total pressure loss versus isentropic Mach number, OV10 cascade $\ldots 136$

Figure 68. Corrected total pressure loss versus isentropic Mach number, Baseline cascade,

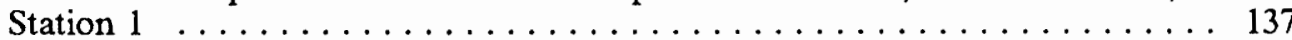

Figure 69. Corrected total pressure loss versus isentropic Mach number, Baseline cascade,

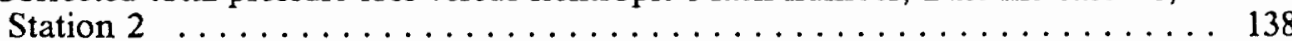

Figure 70 . Overlay of calculated pressure contours and shadowgraph, UN10 $\ldots \ldots \ldots 139$

Figure 71 . Overlay of calculated pressure contours and shadowgraph, Baseline $\ldots \ldots \ldots 140$

Figure 72. Overlay of calculated pressure contours and shadowgraph, OV10 $\ldots \ldots \ldots 141$

Figure 73. Shadowgraph, UN10 cascade, No flow $\ldots \ldots \ldots \ldots \ldots \ldots \ldots \ldots \ldots \ldots$

Figure 74. Shadowgraph, UN10 cascade, $M_{2 i s}=1.02 \ldots \ldots \ldots \ldots \ldots \ldots \ldots \ldots \ldots$ 
Figure 75. Shadowgraph, UN10 cascade, $M_{2 i s}=1.13 \ldots \ldots \ldots \ldots \ldots \ldots \ldots$

Figure 76. Shadowgraph, UN10 cascade, $M_{2 i s}=1.19 \ldots \ldots \ldots \ldots \ldots \ldots$

Figure 77. Shadowgraph, UN10 cascade, $M_{2 i s}=1.24 \ldots \ldots \ldots \ldots \ldots \ldots$

Figure 78. Shadowgraph, UN10 cascade, $M_{2 i s}=1.32 \ldots \ldots \ldots \ldots \ldots \ldots$

Figure 79. Shadowgraph, Baseline cascade, $M_{2 i s}=0.96 \ldots \ldots \ldots \ldots \ldots \ldots$

Figure 80 . Shadowgraph, Baseline cascade, $M_{2 i s}=1.03 \ldots \ldots \ldots \ldots \ldots \ldots \ldots$

Figure 81. Shadowgraph, Baseline cascade, $\mathrm{M}_{2 i s}=1.10 \ldots \ldots \ldots \ldots \ldots \ldots$

Figure 82. Shadowgraph, Baseline cascade, $M_{2 i s}=1.17 \ldots \ldots \ldots \ldots \ldots \ldots \ldots$

Figure 83. Shadowgraph, Baseline cascade, $\mathbf{M}_{2 i s}=1.22 \ldots \ldots \ldots \ldots \ldots \ldots \ldots \ldots \ldots \ldots$

Figure 84. Shadowgraph, Baseline cascade, $M_{2 i s}=1.33 \ldots \ldots \ldots \ldots \ldots \ldots \ldots$

Figure 85 . Shadowgraph, OV10 cascade, No flow $\ldots \ldots \ldots \ldots \ldots \ldots \ldots \ldots$

Figure 86. Shadowgraph, OV10 cascade, $M_{2 i s}=0.95 \ldots \ldots \ldots \ldots \ldots \ldots \ldots \ldots$

Figure 87. Shadowgraph, OV10 cascade, $M_{2 i s}=0.99 \ldots \ldots \ldots \ldots \ldots \ldots \ldots$

Figure 88. Shadowgraph, OV10 cascade, $\mathbf{M}_{2 i s}=1.08 \ldots \ldots \ldots \ldots \ldots \ldots \ldots$

Figure 89 . Shadowgraph, OV10 cascade, $\mathrm{M}_{2 i s}=1.17 \ldots \ldots \ldots \ldots \ldots \ldots \ldots$

Figure 90. Shadowgraph, OV10 cascade, $M_{2 i s}=1.23 \ldots \ldots \ldots \ldots \ldots \ldots \ldots$

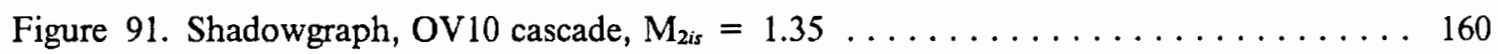




\section{Nomenclature}

Symbols:

$\beta_{m 1} \quad$ Blade inlet metal angle (at camber line)

$\beta_{m 2} \quad$ Blade exit metal angle (at camber line)

$\beta_{1} \quad$ Blade inlet air angle

$\beta_{2} \quad$ Blade exit air angle

$M \quad$ Mach number

$\sigma \quad$ Solidity, $(c / s)$

$s \quad$ Spacing between blades (pitch)

c Blade axial chord

$x \quad$ Horizontal (axial) distance, positive in flow direction from blade inlet

$y \quad$ Tangential distance, positive in direction of blade rotation

$P \quad$ Static pressure

$P_{b} \quad$ Blade base pressure

$\varepsilon \quad$ Blade suction side unguided turning (UGT) or drag-lift ratio

$\delta \quad$ Blade trailing edge wedge angle

$\gamma \quad$ Ratio of specific heats for air

$\Psi_{z} \quad$ Incompressible $Z$ weifel loading coefficient 


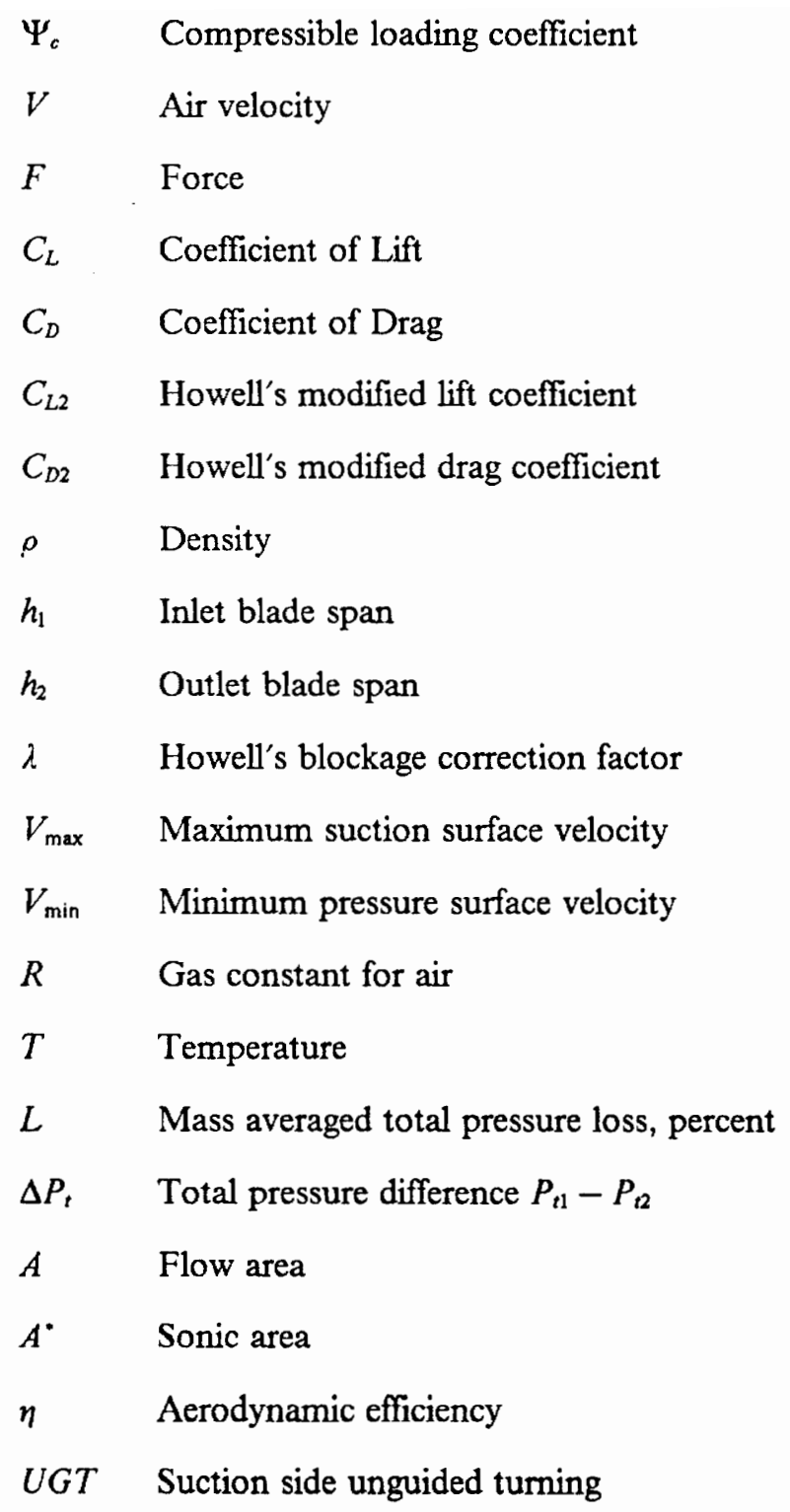

Subscripts:

$0_{1} \quad$ Conditions upstream of the blade row

()exit Conditions at the blade exit plane, applied to $\mathrm{M}, \mathrm{P}$ or $\mathrm{V}$

$\mathrm{O}_{2}$ Conditions at Station $1(x / c=1.17)$, applied to $\mathrm{M}, \mathrm{P}$ or $\mathrm{V}$

() beck Conditions at downstream boundary for computations, $(x / c=1.78)$, applied to $\mathrm{P}$

(is Isentropic conditions 
()t Total (stagnation) condition, applied to $\mathrm{P}$ to $\mathrm{T}$

()T Tangential direction, applied to $\mathrm{F}$

$0_{m} \quad$ Conditions along the blade mean air angle

Oid Ideal

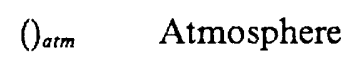

() corrected, applied to $\mathrm{L}$

$\left.()\right|_{\text {STN }}$ Indicates Station 1

$\left.0\right|_{S T N 2}$ Indicates Station 2

() $\left.\right|_{32 c h}$ Indicates data taken with the 32-channel PSI system 


\subsection{Introduction}

The choice of a solidity, or chord to spacing ratio, for turbine blading has an important influence on the effectiveness of the blade row. A minimum value of solidity (large blade spacing) is desired to reduce engine weight and cost and to minimize the amount of cooling flow required, since increased cooling reduces efficiency. Solidity reductions, however, are limited by mechanical considerations due to increased blade loading, and by aerodynamic considerations since increased loading tends to cause suction side flow separation, hence increased loss, on turbine blades.

There are two standard solidity definitions - true solidity and axial solidity. The difference is the use of either the blade true chord or axial chord in the chord to pitch ratio. The two definitions can be interchanged by taking into account the stagger angle of the blade. For this investigation, the axial solidity definition will be used for convenience.

Standard industry practice for many years has been to use the Zweifel coefficient $\Psi_{z}$, which relates blade loading to axial solidity, to choose an optimum solidity. As the result of subsonic cascade experiments conducted in the 1950's, Zweifel suggested that a $\Psi_{2}$ value of about 0.8 should give optimum blade performance. Since then there have been many analytical and experimental investigations of solidity, resulting in different recommendations for the value of $\Psi_{z}$. Current aircraft industry design guidelines recommend $\Psi_{z}=1.1$ for transonic, high pressure ratio turbine 
rotor blades, and $\Psi_{z}=0.5$ to 0.7 for the corresponding nozzle blades. It is interesting to note that computation and experiment seldom agree well on a choice of optimum solidity. This is because there are so many factors that influence optimum solidity, such as blade curvature, reaction and inlet and exit air angles. It is the intent of this investigation to determine whether the optimum solidity of the blade tested was correctly chosen using the analytical tools available to GEAE turbine designers.

There are three typical experimental methods for investigating solidity effects on blade losses. The most complicated and most expensive is a full rig test, where blades with different solidities are carefully designed to have nearly identical velocity profiles and loadings. However, these tests are complicated by other effects such as changes in blade reaction, secondary flows, and other unexpected consequences due to different blade designs. The second method is to increase the spacing and the thickness of blades simultaneously without changing the blade chord or surface shape. While this method provides a constant throat area and thus identical mass flows between cases, blade aerodynamics becomes a complicating variable. For example, the trailing edge thickness of the blades changes for different solidities, which has a large effect on the blade performance. The third method, used in this investigation, involves no blade redesign. Instead the blade spacing is changed and the blade stagger is then varied to keep a constant throat to spacing ratio. Consequently, the blade exit air angles are very similar, as well as the blade aerodynamics. The drawback of this method is that the blade shape cannot be optimized at different solidities. A variation of this method is to simply change the blade spacing with no change in the stagger. This causes large changes in the passage flow and complicates a determination of the effect of solidity. 


\subsection{Solidity Relations}

\subsection{Lift and Drag Coefficients}

The simplest relations between blade solidity and blade forces are the lift and drag coefficients. The lift force acts on a blade perpendicular to the direction of the mean velocity $V_{m}$, and the drag acts parallel to the mean velocity. The mean velocity is in the direction $\beta_{m}$ defined by $\tan \beta_{m}=\frac{1}{2}\left(\tan \beta_{1}+\tan \beta_{2}\right)$. See Figure 1 for the definitions and senses of blade velocity angles. Combining these definitions in the tangential direction (the direction of blade rotation) gives $F_{T}=L \cos \beta_{m}-D \sin \beta_{m}$. Employing the definitions of lift and drag coefficient, the blade force in the tangential direction becomes

$F_{T}=\frac{1}{2} \rho C_{L} c V_{m}^{2} \cos \beta_{m}-\frac{1}{2} \rho C_{D} c V_{m}^{2} \sin \beta_{m}$

From the momentum equation for steady, inviscid flow, the blade force in the tangential direction is

$F_{U}=\rho s\left(V_{1}^{2} \cos \beta_{1} \sin \beta_{1}-V_{2}^{2} \cos \beta_{2} \sin \beta_{2}\right)$ 
Equating $F_{U}$ and $F_{T}$, which must be equal, gives a relation between the force coefficients,

$$
\frac{C_{L} \cos \beta_{m}-C_{D} \sin \beta_{m}}{\cos ^{2} \beta_{m}}=2 \frac{s}{c}\left(\tan \beta_{1}-\tan \beta_{2}\right) .
$$

For inviscid, incompressible flow, $\mathrm{D}$ is zero and the lift coefficient becomes

$$
C_{L}=2 \frac{s}{c}\left(\tan \beta_{1}-\tan \beta_{2}\right) \cos \beta_{m}
$$

In reality the blade drag is a small percentage of the lift and the above formula for the lift is often used. An expression for the drag coefficient is obtained by a pressure balance along the mean velocity direction,

$$
C_{D}=\frac{s}{c} \frac{P_{1}-P_{\text {exit }}}{\frac{1}{2} \rho V_{1}^{2}} \cos \beta_{m}
$$

\subsection{The Zweifel Coefficient}

The lift coefficient $C_{L}$, then, relates blade loading to blade solidity. However, no useful correlation between loading and solidity can be obtained using $C_{L}$ because it varies widely with incidence angle, which is not a constant value in an operating turbine. Zweifel therefore attempted to find another expression relating blade loading to solidity which would provide a better correlation. He did this by comparing the actual blade pressure distribution to an ideal blade loading. The ideal blade loading was defined as $P_{t 1}$ acting over the entire pressure surface, and $P_{\text {exit }}$ acting over the entire suction surface (see Figure 2). For an axial turbine blade of arbitrary inlet $\operatorname{span} h_{1}$ and outlet span $h_{2}$, assuming a trapezoidal blade shape in the meridional plane, the ideal tangential force is therefore 
$F_{T, i d}=c\left(P_{t 1}-P_{e x i t}\right)\left(\frac{h_{1}+h_{2}}{2}\right)$

For the same arbitrary blade the actual tangential force is

$F_{T}=\rho s\left(h_{1} V_{1}^{2} \sin \beta_{1} \cos \beta_{1}+h_{2} V_{\text {exit }}^{2} \sin \left|\beta_{2}\right| \cos \beta_{2}\right)$

Creating the ratio of actual to ideal blade force in the tangential direction results in the tangential Zweifel coefficient

$\Psi_{z}=\frac{F_{T}}{F_{T, i d}}=\rho s \frac{\left(h_{1} V_{1}^{2} \sin \beta_{1} \cos \beta_{1}+h_{2} V_{e x i t}^{2} \sin \left|\beta_{2}\right| \cos \beta_{2}\right)}{c\left(\frac{h_{1}+h_{2}}{2}\right)\left(P_{t 1}-P_{e x i t}\right)}$.

Making the assumption of incompressible flow, Bernoulli's equation can be applied and

$P_{t 1}=P_{\text {exit }}+\frac{1}{2} \rho V_{\text {exit }}^{2}$

Thus,

$\Psi_{2}=2\left(\frac{s}{c}\right)\left[\left(\frac{2}{h_{1}+h_{2}}\right) h_{1}\left(\frac{V_{1}}{V_{\text {exit }}}\right)^{2} \sin \beta_{1} \cos \beta_{1}+h_{2} \sin \left|\beta_{2}\right| \cos \beta_{2}\right]$

Finally, substituting in mass continuity,

$\frac{V_{1} \cos \beta_{1}}{V_{\text {exit }} \cos \beta_{2}}=\frac{h_{2}}{h_{1}}$

a useful form of the Zweifel coefficient is arrived at:

$\Psi_{z}=\frac{4\left(\frac{s}{c}\right) \cos \beta_{2}\left[\left(\frac{h_{2}}{h_{1}}\right) \tan \beta_{1} \cos \beta_{2}+\sin \left|\beta_{2}\right|\right]}{\left[1+\left(\frac{h_{1}}{h_{2}}\right)\right]}$ 
For a linear cascade $h_{1}=h_{2}$ and the equation reduces to:

$\Psi_{z}=2\left(\frac{s}{c}\right) \cos \beta_{2}\left(\tan \beta_{1} \cos \beta_{2}+\sin \left|\beta_{2}\right|\right)$

Note that this form is very similar to the lift coefficient $C_{L}$ but is based on $V_{2}$ rather than $V_{m}$.

An interesting limit of the Zweifel coefficient occurs for impulse blades, when $\beta_{1}=-\beta_{2}$. For this case,

$\Psi_{2}=2\left(\frac{s}{c}\right) \sin \left(2\left|\beta_{2}\right|\right)$

For a $\Psi_{z}$ value of 0.8 this is identical to Briling's rule, which is usually stated as

$\left(\frac{c}{s}\right)=2.5 \sin \left(2\left|\beta_{2}\right|\right)$

Briling's rule, derived from the geometry of a blade passage with constant width and curvature, gives the optimum spacing for impulse blades with inviscid subsonic flow. The factor 2.5 comes from a rule of thumb for minimum loss observed by Briling. A similar formula based on Briling's rule is applicable for fully supersonic impulse blade flows when transition regions are added to both the blade inlet and outlet. These transition regions are necessary to decelarate and accelerate the pressure side Mach number to match the flow conditions in the constant width and curvature section, where the suction side Mach number is higher. Reference 3 gives a good outline of the uses for Briling's rule. What is important here is that a single value of $\Psi_{z}$ represents Briling's rule for all values of $\beta_{1}=\left|\beta_{2}\right|$. This suggests that Zweifel's ratio of actual to ideal loading is useful.

In his paper (Ref. 40), Zweifel plots the relationship of the drag-lift ratios $(\varepsilon)$ of several subsonic cascades to both the coefficient of lift and the Zweifel loading coefficient (Figure 3). Note that $C_{A}$ and $\Psi_{A}$ shown on the figures indicate the lift and Zweifel coefficients perpendicular to $V_{m}$, which can be derived similarly to $C_{L}$ and $\Psi_{z}$. The curves show that the minimum drag occurs over a wide range of $C_{A}$ values but is confined to $\Psi_{A}$ values between 0.9 and 1.0 , which corresponds to a $\Psi_{z}$ of 
about 0.8. Thus, given the turning requirements for a blade row in the subsonic regime, the optimum solidity can be estimated using the Zweifel coefficient. Note that values of $\Psi_{z}$ greater than one are possible for highly loaded blades, where the suction surface static pressure may be lower than the exit static pressure along much of the chord.

\subsection{Howell's Relationship}

Independent of Zweifel's work, a similar correlation was obtained by Howell (Ref. 19). Again using the blade exit velocity rather than the mean velocity, Howell proposed two new coefficients related to the lift and drag coefficients as follows:

$C_{L 2}=C_{L}\left(\frac{V_{m}}{V_{2}}\right)^{2}=C_{L}\left(\frac{\cos ^{2} \beta_{2}}{\cos ^{2} \beta_{m}}\right)$

and

$C_{D 2}=C_{D}\left(\frac{V_{m}}{V_{2}}\right)^{2}=C_{D}\left(\frac{\cos ^{2} \beta_{2}}{\cos ^{2} \beta_{m}}\right)$.

In addition, a factor $\lambda$ was included to compensate for the blockage effect of blade passage boundary layers, which lowers lift and increases drag at high values of solidity. This factor was defined as follows:

$\lambda=\left[\frac{6\left(\frac{s}{c}\right)-1}{\left(\frac{s}{c}\right)}\right]$

Howell then plotted (Figure 4) $\lambda C_{D 2}$ against $C_{L 2} / \lambda$ for some subsonic turbines and compressors. The figure shows minimum drag for $C_{L 2} / \lambda$ values between 1.0 and 1.3. Note that Howell plotted 
both actual cascade data as well as data deduced from rig tests with allowances made for secondary flow losses and other types of loss. Howell also proposed a curve fit for this data,

$\lambda C_{D 2}=0.15\left(1+\frac{C_{l 2}}{\lambda}\right)\left(\frac{C_{L 2}}{\lambda}-1\right)^{2}+0.016\left(\frac{C_{L 2}}{\lambda}\right)$

In proposing this fit Howell took into account the fact that compressors stall and surge in the high-lift, high-drag region and that Mach number effects reduce the loss in the low-lift range, so that the correlation could be applied to both subsonic compressors and turbines. Thus, once blade turning angles are determined, the modified lift and drag coefficients can be calculated and Howell's relationship used to compute the blade solidity.

\subsection{Diffusion Parameters}

Zweifel's coefficient ties together several blade performance parameters in an attempt to predict optimum solidity. However, the relationships between parameters other than solidity and loading are not made clear by Zweifel's ideal model. Three blade characteristics not explicitly represented in the Zweifel coefficient are blade turning $\left(\beta_{1}-\beta_{2}\right)$, blade reaction, and blade surface velocity diffusion. Stewart and Glassman explored the effects of these parameters on optimum solidity in the late 1960's (Ref. 34). Instead of dividing the blade lift coefficient by Zweifel's ideal distribution, Stewart and Glassman used a distribution based on $V_{\max }$ and $V_{\min }$, which are the maximum suction surface velocity and minimum pressure surface velocity, respectively. This change extends Zweifel's model to highly-loaded (but still subsonic) blade cascades where a large portion of the suction surface velocity may be greater than the exit velocity. It also allows the use of suction and pressure side diffusion parameters, defined as 
$D_{s}=\frac{V_{\max }^{2}}{V_{2}^{2}}$

and

$D_{p}=\frac{V_{1}^{2}}{V_{\min }^{2}}$.

These parameters represent the amount of velocity decrease (or pressure rise) along the blade surfaces, which determines whether the flow will separate from the blade. These are combined with the reaction,

$R=1-\frac{V_{1}^{2}}{V_{2}^{2}}$

to give the overall diffusion parameter $D$, defined as the sum of the changes in kinetic energy on the suction and pressure surfaces divided by the blade outlet kinetic energy:

$D=\frac{V_{\max }{ }^{2}-V_{2}{ }^{2}+V_{1}{ }^{2}-V_{\min }{ }^{2}}{V_{2}{ }^{2}}$.

Using the above definitions,

$D=D_{s}-\frac{(1-R)}{D_{p}}-R$

and Zweifel's coefficient becomes

$\Psi_{2}=\frac{2}{\sigma} \frac{\sqrt{1-R}}{D+R} \sin \left(\beta_{1}-\left|\beta_{2}\right|\right)$

This relationship is plotted at the top of Figure 5 as an axial solidity parameter, 
$\frac{\sigma \Psi_{z}}{\sin \left(\beta_{1}-\left|\beta_{2}\right|\right)}$

versus reaction $R$.

Thus if the value of $\Psi_{z}$ is known, the solidity can be predicted from blade reaction and diffusion, which are in turn determined by the blade velocity triangles. However, it is not clear how reaction, turning, and diffusion affect the value of $\Psi_{z}$. Stewart and Glassman computed the effect of these variables on the loading coefficient by assuming a typical profile for a highly loaded subsonic turbine blade. From this, they determined that the effects of $\beta_{2}, \beta_{1}-\left|\beta_{2}\right|, D_{s}$ and $D_{p}$ on the load coefficient were of an order less than the effect of reaction. Thus, they predict the loading coefficient as follows:

$\Psi_{z}=0.6 \sqrt{1-R^{2}}+0.15$

This relationship was then incorporated into the axial solidity parameter to give the plot at the bottom of Figure 5. From this figure, the blade solidity can be determined from the turning, reaction, and diffusion. Thus Stewart and Glassman put forth a more detailed method for determining optimum solidity than that suggested by Zweifel.

\subsection{Other Relations}

Kuofang and Naixing (Ref. 24) proposed a parameter defined as

$Q=\frac{\cos \beta_{1}}{\cos \beta_{2} \sin \left(\beta_{1}+\left|\beta_{2}\right|\right)}$, 
which they then used to correlate subsonic cascade data. By fitting an average curve through the available data, they determined a relationship for optimum pitch-chord ratio,

$$
\left(\frac{s}{c}\right)_{o p t}=0.09 Q+0.46
$$

The reasoning behind the definition of $Q$ is not clear from Kuofang and Naixing's presentation, nor is it obvious why they knew that their test points were at optimum solidity. It is clear that the correlation is intended only for subsonic turbine cascades.

Baines and Oldfield (Ref. 2) modified Zweifel's analysis to include blade flows that are not incompressible, but still isentropic, for the special case of $\beta_{1}=0$. Starting with Zweifel's ratio of actual to ideal loading, which reduces to

$\Psi_{z}=\frac{s}{c} \cos ^{2} \beta_{2} \tan \left|\beta_{2}\right|\left(\frac{\rho V_{2}{ }^{2}}{P_{t 1}-P_{\text {exit }}}\right)$

for $\beta_{1}=0$, and introducing the ideal gas law, results in

$$
\Psi_{z}=\frac{s}{c} \cos ^{2} \beta_{2} \tan \left|\beta_{2}\right|\left(\frac{\gamma M_{2 i s}{ }^{2} P_{e x i t}}{P_{t 1}-P_{e x i t}}\right) .
$$

Assuming isentropic flow $\left(P_{t 1}=P_{t, e x i t}\right)$, but not assuming incompressibility, and expanding gives

$\Psi_{c}=\frac{s}{c} \cos ^{2} \beta_{2} \tan \left|\beta_{2}\right| F(M)$,

where

$$
F(M)=\frac{\gamma M_{2 i s}^{2}}{\left\{\left[1+\left(\frac{\gamma-1}{2}\right) M_{2 i s}{ }^{2}\right]^{\left(\frac{\gamma}{\gamma-1}\right)}-1\right\}} .
$$


For incompressible flow $(M \simeq 0), F(M)=2$ and the expression reduces to Zweifel's original form. However, for higher Mach numbers $F<2$. Note that Baines and Oldfield made no attempt to predict the optimum solidity based on this modified coefficient. However, the relationship between the compressible loading coefficient $\Psi_{c}$ and the incompressible form $\Psi_{z}$ is given by:

$\frac{\Psi_{c}}{\Psi_{z}}=\frac{F(M)}{2}$

This relationship can be used to get an idea of the effect of compressibility on the loading coefficient. Although the above is for axial inlet blades only, a more general expression could by derived for all inlet angles, but the mathematics would be more difficult. 


\subsection{Previous Research}

The effect of a solidity change on subsonic turbine blading is well established, as shown in the previous chapter. However, a method for determining the optimum solidity of transonic blading is not yet established and its development, if possible, is not likely to be straightforward. Currently the problem of determining the optimum is approached experimentally. There are several experimental methods which have been used, as outlined in the Introduction. In this chapter some past experiments on the effect of solidity of transonic blading will be outlined. In general the results are inconclusive and reveal little about a reliable method to design transonic blades for optimum solidity. The examples used here are simply ones the author came upon in his research and do not necessarily represent a definitive selection of solidity experiments.

\subsection{Stearns}

The first of several solidity investigations at G. E. Aircraft Engines considered here was reported by Stearns (Ref. 33). This was a full-scale rig test of a single-stage high pressure turbine. The rotor blades, of interest here, were twisted with subsonic inlet and exit, and had maximum loading at the 
pitchline. Although the exit flow was subsonic, super-critical velocities probably occurred at the throat for high pressure ratios, placing the turbine at the low end of the transonic range. No Mach number profiles were provided by Stearns to verify this. The blade solidity was increased and decreased by ten percent by simply re-spacing the blades, with no change in blade orientation. The results were as follows:

\begin{tabular}{|cccc|}
\hline$\sigma$ & $\Psi_{z}$ & $\boldsymbol{\eta}$ & UGT \\
\hline 1.36 & 0.99 & 0.88 & $21^{\circ}$ \\
1.28 & 1.09 & 0.87 & $24^{\circ}$ \\
1.15 & 1.21 & 0.86 & $27^{\circ}$ \\
\hline
\end{tabular}

Thus the efficiency was found to increase with an increase in solidity. This suggests that the optimum loading would occur for a $\Psi_{2}$ value less than 0.99 , which is unexpected for transonic blading. Generally, a value of 1.1 for the Zweifel loading coefficient is used in the aircraft industry when designing transonic turbine rotors. The unexpected results were attributed to a decrease in reaction with a decrease in solidity, as well as an increase in unguided turning. It is an established design practice for blades of this type to keep the suction side unguided turning small to minimize losses by decreasing the trailing edge shock strength. This particular nozzle also exhibited flow separation from the suction side for large values of unguided turning. The decrease in reaction with decreasing solidity is caused in this experiment by a decrease in the ratio of exit area to throat area, a consequence of spacing the blades with no other adjustments. All of these effects could not be separated to make a valid conclusion about the effect of solidity change alone. 


\subsection{Cherry and Thomas}

Cherry and Thomas (Ref. 5) attempted a more ambitious investigation of solidity, designing seven different blades for planar cascade studies. The blades typified rotor airfoils for single-stage high pressure ratio turbines. The blades were all designed to have an inlet Mach number of about 0.6 , an exit Mach number of about 1.15, inlet metal angle of about 50 degrees, and exit metal angle of -62 degrees. That is, the blade shapes and spacing were changed but the velocity triangles remained the same. Values of Zweifel loading coefficient of 1.0, 1.1, 1.2, and 1.3 were used, and axial solidities in the range 1.14 to 1.56 . The unguided turning varied from 10 to 15 degrees. The cascade efficiency was measured for a wide range of pressure ratios.

The effect of solidity change was inconclusive because of the many other factors other than solidity introduced by the different blade designs. The difficulty encountered by the interaction between variables influencing blade loss is one of the factors which makes determination of an optimum solidity rule so difficult.

\subsection{Lemond, Thomas, and Walker}

The final GEAE investigation to be considered here was reported by Lemond, Thomas, and Walker (Ref. 25). Two annular cascades of transonic stators were compared for the effect of solidity. The two cascades had different blade profiles designed for the same vector diagram of a high pressure, single stage turbine. The number of blades in the annulus, and therefore the blade spacing, was held constant. The blade chord was reduced to reduce the solidity. This method allowed the following blade characteristics to remain unchanged as the solidity was reduced: throat size, blade pitch, unguided tuming, and trailing edge blockage. The cascade was also tested using fully cooled 
blades. The two solidities tested were $\sigma=0.87$ and $\sigma=0.68$, with the following blade characteristics: $\beta_{m 1}=0^{\circ}, \beta_{m 2}=-75^{\circ}, s=4.0 \mathrm{~cm}$, and $U G T=8.2^{\circ}$. The Baseline solidity $(\sigma=0.87)$ case had an axial chord of $3.4 \mathrm{~cm}$ and a Zweifel loading coefficient of 0.6 . The low solidity blade had $c=2.7 \mathrm{~cm}$ and $\Psi_{z}=0.77$. These characteristics, combined with a Mach number distribution similar to that used in the current investigation, make the experiment very similar to that described in this thesis.

The efficiencies of the cascades were measured for a wide range of operating pressure ratios. The results indicated that the pitchline efficiency was unaffected by decreasing the solidity, while the hub and tip losses were increased by the solidity decrease. The overall effect on the blade was a lowered efficiency due to the 22 percent decrease in solidity. Specifically, the pitchline efficiency was about 96 percent at the design pressure ratio, the overall efficiency was about 93 percent for the high solidity, and the overall efficiency was 91.5 percent for the low solidity. For lower pressure ratios, the efficiencies increased by the same trend.

There were several major differences between the blade Mach number distributions which may have masked the effect of the solidity change. For example, the high solidity case had a large suction side diffusion near the trailing edge, while the reduced solidity case had less diffusion but a greater shock/boundary layer interaction on the suction side. Also, the peak suction side Mach number and exit Mach number varied between the two blade designs. These differences are all significant in terms of the blade performance, and thus make it difficult to isolate the effect of solidity.

\subsection{Plohr and Hauser}

Plohr conducted several solidity investigations at NACA in the 1950's for two dimensional cascades of transonic airfoils. His aim was to reduce the solidity of highly loaded turbine airfoils suitable for 
air cooling. Two investigations will be discussed here. For the first (Ref. 15), the spacing of a pitchline section of an air-cooled, non-twisted rotor blade was increased to decrease the solidity from $\sigma=1.38$ to $\sigma=1.06$. The corresponding Zweifel numbers were $\Psi_{z}=0.9$ and $\Psi_{z}=1.16$. The following blade characterisitics were in common between the two solidities: $\beta_{m 1}=36.2^{\circ}$, $\beta_{m 2}=-60^{\circ}$, and $c=3.6 \mathrm{~cm}$. The exit and suction side peak Mach numbers were roughly identical for the two blades. However, the particular blade that Plohr tested was plagued with a suction side separation, especially at low pressure ratios. At higher pressure pressure ratios the separation decreased, until the flow was completely attached when the blade was fully loaded. The separation was worse for the low solidity blade, causing a greater amount of relative turning for that case. It is of interest that this blade had a very highly loaded leading edge, which certainly was the primary cause for flow separation.

The experiment found a lower efficiency for the low solidity case at low pressure ratios, and comparable efficiencies between the two blades closer to limit load. The efficiency was expressed as the square of the ratio of actual to isentropic velocity (the velocity coefficient) of fully mixed flow, obtained using continuity beyond the trailing edge of the blade. The specific results were as follows:

\begin{tabular}{|ccc|}
\hline $\boldsymbol{\sigma}$ & $\boldsymbol{\eta}, \boldsymbol{M}_{\mathbf{2}}<\mathbf{1}$ & $\boldsymbol{\eta}, \boldsymbol{M}_{\mathbf{2}}>\mathbf{1}$ \\
\hline 1.06 & 0.92 & 0.98 \\
1.38 & 0.96 & 0.98 \\
\hline
\end{tabular}

Thus, due to the separation for low Mach numbers, the decreased solidity caused an efficiency penalty. However, as limit loading was approached the decreased solidity appeared to cause no efficiency decrease. 


\subsection{Plohr and Nusbaum}

The blades used for Plohr's second experiment (Ref. 29) were better designed, and had no large flow separations. Solidity was reduced from $\sigma=2.16\left(\Psi_{z} \simeq 0.7\right)$ to $\sigma=1.68\left(\Psi_{z} \simeq 0.9\right)$ by increasing the blade spacing. The blades had the same geometry, with $\beta_{m 1}=43.5^{\circ}, \beta_{m 2}=-55.8^{\circ}$, and $c=4.6 \mathrm{~cm}$. The reduced solidity raised the velocity coefficient by about 0.2 for all pressure ratios. That is, the loss was decreased. A plateau of maximum velocity coefficient was found to occur for $P_{t} / P_{\text {exit }}$ ratios between 2.4 and 2.6, where 2.6 represents limit loading. In his report, Plohr gave no indication of the relationship between the blade Mach number profiles for the two different spacings. Plohr attributed the increased efficiency with decreased solidity to reduced wake loss due to fewer blades in the row.

\subsection{Oldfield}

The best approach to solidity change in turbine blade design was shown by Oldfield (Ref. 2). He reports on a program at Oxford to reduce the solidity of an established Rolls-Royce turbine blade. Two new blades with solidity reduced by thirteen and twenty percent were designed, with the intent to match the efficiency of the original blade. The solidity was decreased by increasing the blade spacing with constant chord, but adjustments were made to the blade curvature to compensate for undesirable flow characterisitics. Also, the blade throat to spacing ratio was kept constant, a detail overlooked in all of the above experiments. Although many details of the blade geometry are not given in Oldfield's paper, the blades all had an exit metal angle of -65.2 degrees and a design isentropic exit Mach number of 0.995 . The solidity values used were 1.49, 1.30, and 1.19. Also, all of the blades had the same amount of unguided turning, since all of the changes in blade 
geometry were made upstream of the throat. That is, only the loading of the leading edge up to the throat was changed.

This approach was successful in reducing the blade solidity without increasing the profile loss. In fact, the design point efficiency was actually improved. The solidity reduction also changed the character of the loss curve, creating a dip in the loss at the design point. Oldfield was able to explain the different charactersitics of the loss curves with detailed measurements of boundary layer transition and shape using heated thin films and a flattened pitot probe. The approach used by Oldfield and Rolls-Royce is, in this author's opinion, the best method currently available to design for optimum solidity. That is, start with an accepted blade design and attempt to reduce the solidity, keeping as many things constant as possible and paying close attention to details of the flow. A general rule for optimum solidity in the transonic regime seems far off, and although Oldfield's method is time consuming and costly, it is most likely to work. 


\subsection{Description of NOVAK2D}

As part of the preliminary design for this work a computational analysis of the flowfields was performed for the different solidities. This was done using a time-marching code owned by GEAE called NOVAK2D. An overview of the capabilities and usage of this code will be given below, with the emphasis placed on its use for the type of flow problem represented by this solidity investigation. More detailed information can be found in References 16 or 17.

NOVAK2D uses an explicit fourth order Runge-Kutta time marching, finite volume scheme to solve the Navier-Stokes equations. It can be used in flow regimes from subsonic to hypersonic, and can solve two-dimensional, quasi-3D or axisymmetric flow problems. In these modes the effects of blade radius, lamina (streamsheet) thickness, and blade row rotation can be considered. The code was designed to be used for inviscid and viscid laminar or turbulent flow problems. However, at the time it was used for this experiment, only the inviscid mode was working properly. The rest of this discussion, then, will focus on the inviscid capabilities of NOVAK2D.

Planar cascades of turbine blades, such as those for this thesis, require a quasi-3D computational code. For purposes of calculation, the cascades were represented as turbine blade rows with a pitchline radius of of 25.3 meters, a blade span of $15.2 \mathrm{~cm}$, and 4,282 blades. Observed in a meridional plane ( $\mathrm{x}$ is axial, $\mathrm{y}$ is tangential), such a blade row would be nearly linear in the 
tangential direction. Since all of GEAE's blade row flow calculation codes can only handle meridional geometry, the large radius was necessary to simulate a truly linear cascade.

The general form of the Euler equations for quasi-3D flow is given below, in conservative form. These equations were derived from the three-dimensional form on an axisymmetric stream surface with radius $r$ from the axis of rotation, thickness $b(x)$, and angular velocity of the coordinate system $\Omega$. The relative velocities are $w_{x}$ and $w_{y}$, the specific internal energy $\mathrm{e}$, and the rothalpy $\mathrm{h}$. The equations are given by:

$\frac{\partial U}{\partial t}+\frac{\partial F}{\partial x}+\frac{\partial G}{\partial y}=H$

where

$U=r^{2} b\left[\begin{array}{c}\rho \\ \rho w_{x} \\ \rho w_{y} \\ \rho e\end{array}\right] \quad F=r b\left[\begin{array}{c}\rho w_{x} \\ \rho w_{x}^{2}+P \\ \rho w_{x} w_{y} \\ \rho w_{x} h\end{array}\right]$

$G=r b\left[\begin{array}{c}\rho w_{y} \\ \rho w_{x} w_{y} \\ \rho w_{y}{ }^{2}+P \\ \rho w_{y} h\end{array}\right] \quad H=\left[\begin{array}{c}0 \\ P \frac{\partial(r b)}{\partial x}+\rho b\left(w_{y}+\Omega r\right)^{2} \frac{\partial r}{\partial x} \\ -\rho b w_{x}\left(w_{y}+2 \Omega r\right) \frac{\partial r}{\partial x} \\ 0\end{array}\right]$

and

$e=\frac{P}{(\gamma-1) \rho}+\frac{1}{2}\left(w_{x}^{2}+w_{y}^{2}-\Omega^{2} r^{2}\right)$

$h=\frac{\gamma P}{(\gamma-1) \rho}+\frac{1}{2}\left(w_{x}{ }^{2}+w_{y}{ }^{2}-\Omega^{2} r^{2}\right)$. 
For the inlet boundary conditions, the internal energy and rothalpy are fixed, which implies a fixed inlet total pressure. Also, the inlet tangential velocity or the absolute inlet flow angle is fixed. At the outlet, the static pressure is specified by the user, and the flow direction, density, and internal energy are calculated by the time-marching scheme. If NOVAK2D is to be used in purely two-dimensional mode, $\mathrm{r}$ is set to one, $\mathrm{b}$ is constant, $\Omega$ is zero, and $\mathrm{x}$ and $\mathrm{y}$ become the usual cartesian coordinates. For this research, the quasi-3d mode with $\Omega=0$ was used.

The feature which makes NOVAK2D unique and very useful to a turbine design engineer is an adaptive grid capability. Unlike most CFD codes, the user does not have to input an intelligent grid to get a correct flowfield solution. The grid-generation routine in NOVAK2D can fit a grid to any arbitrary geometry, even complicated features such as struts in a blade passage and bypass slots. This is done by first "carpet-bombing" the area of interest with an equilateral array of points. Then a triangulation routine is used to fit an optimum array of triangles to those grid points. The algorithm used is called Delauney triangulation, which defines triangles such that they are all as close to equilateral as possible. Next the first of two grid adaptations is done; the grid points are refined based on the curvature of the geometric boundaries of the flowfield. Points nearest to the boundaries are moved to the boundary, outside points are deleted and points are added as needed to make a complete set of triangles at the boundary. The solver is then applied to these finite volumes. After a specified time step (a default is supplied), each triangle is checked for the range of pressure, velocity, and total pressure among its nodes. If the range of any one property exceeds a specified limit, then grid refinement takes place at that triangle. The triangle is subdivided in one of several ways into smaller triangles. The solver then proceeds with another time step and the refinement process is again used. This continues until the specified number of time steps is reached or the smallest triangles reach a specified minimum size. The limit on triangle size is necessary to prevent infinite refinement at shock waves, which have nearly discontinous flow properties. The triangular grid refinement allows the solver to resolve small-scale flow features to arbitrary refinement. 
For an engineer at GEAE to use NOVAK2D, all that is required for input is a blade geometry file which also lists the desired outlet static pressure and inlet flow conditions. The geometry file is linked with an input file listing the default values of all the adjustment variables, and then input to the NOVAK2D solver, currently run on a CRAY XMP with a VAX as front-end. In some cases, the solution does not converge on the first try and adjustments to the default variables must be made. The convergence is represented by the output variables EMAX and EAVE, listed on any output (see Figure 16), which are the maximum and average change in node properties since the last time step. Another indication of the convergence is the number of nodes, which should be roughly the same for separate calculations of similar geometries, for example the three different solidities calculated here. An excessive number of nodes likely indicates that the grid refinement has gotten carried away and the sensitivity of the refinement to blade trailing edge curvature (a likely trouble spot) or similar default variable must be adjusted. Similar to the number of nodes is the number of triangles formed, also listed, along with the number of quadrilaterals. For inviscid cases there are no quadrilaterals used. They are used in the expected boundary layer regions of viscid calculations to give a good grid density in the flow direction but a sparse density across the flow, which minimizes calculation time. In general, EMAX and EAVE should be of the order $10^{-2}$ or less.

A post-processing program is used to get boundary and contour plots of the calculated flow properties, as well as derived quantities such as entropy. Pressure contours are shown in the appendix for the cascades used in this study (Figure 16), as well as blade surface Mach number plots (Figure 14). Because of the inviscid nature of the solution, two cautions are necessary when looking at the pressure and Mach number results. First, since the result is inviscid, the shock strengths and spatial spread of the shocks, as well as base pressure, will not be correctly predicted. Although trends between the differecnt geometries will be correct, the absolute level will be wrong. In particular, shock strengths will be too high because of the lack of viscous dissipation, and the shocks be spatially concentrated. Base pressures will be predicted too high. Also, the spatial spread of pressure rise across shocks will vary depending on the degree of refinement of the final solution 
grid. Note that the pressure difference across the shock will not be affected by the grid, just the spread of that pressure difference. Finally, for turbine blade inviscid calculations, there are two Mach number peaks at the trailing edge, higher than the suction side exit Mach number. This is due to the sudden expansion around the trailing edge, which is not a physically realistic phenomenon. In reality, the flow separates on both sides of the trailing edge and bypasses a region of viscous eddies, which enforces the Kutta condition. In the inviscid case the flow follows the curvature of the trailing edge and the Kutta condition is enforced by numerical dissipation. More will be said about the computational results specific to this research later in this paper. 


\subsection{Experimental Design}

\subsection{Blade Geometry}

The established aim of this research was to find out the sensitivity of the turbine blade being studied at VPI to changes in solidity. Stated another way, the goal was to discover if the blade was at its optimum solidity. At the same time, the experiment was intended to be quick and inexpensive, a "first look" at the solidity question for this transonic turbine blade. As discussed in the Introduction and Previous Research chapters, there are many ways to investigate the effect of solidity on blade row efficiency. In this case, to keep time and cost low, no new blades were designed. Thus, the only way to change the solidity was to re-space the blades. While this method does not allow exact matching of the blade Mach number profiles, it is approximate and much easier than methods involving blade redesign. However, it is seen from other experiments that simply changing the spacing causes other effects, such as change in reaction and unguided turning, which can make it difficult to separate out the effect of solidity.

To compensate for the change in reaction caused by a change in blade spacing, the blade throat to spacing ratio was keep constant as the solidity was changed. The advantage of this can be looked 
at in two ways, in terms of air exit angle and blade reaction. First, a crude way of estimating the air exit angle from a cascade is given by simple geometry as

$\cos \beta_{2}=\frac{t}{s}$

While it is true that exit angle varies widely over a blade pitch, this expression gives an indication of the average. Thus by keeping the throat to spacing $(t / s)$ ratio constant when the blade spacing is changed, the flow exit angle is kept as constant as possible without blade re-design, and the change in exit flow characterisitics is reduced. Second, consider the blade reaction in the form

$R=1-\frac{V_{1}^{2}}{V_{2}^{2}}$

which was used in Reference 34. Note that this definition is based on the change in kinetic energy through the cascade rather than the customary definition relating rotor static enthalpy change to stage work. The reaction definition given above is convenient for a linear cascade. Now consider an increase in the blade spacing, $s$. The throat $t$ must then necessarily increase also. However, because of the suction side curvature of a turbine blade, $t$ increases more than $s$, and the throat to spacing ratio increases. The $t / s$ ratio is analogous to the $A / A^{*}$ familiar from nozzle design, where $A^{\bullet}$ is the sonic area. Thus when $t / s$ increases, $A / A^{*}$ decreases, the blade exit velocity decreases, and the blade reaction decreases. Thus if the blade solidity is decreased by simply increasing the spacing, the reaction decreases, and vice-versa. The change in reaction can be prevented, however, by keeping the throat to spacing ratio constant, so that $A / A^{\circ}$ is constant, and the exit velocity is unchanged.

For this experiment the throat to spacing ratio was kept constant by simply rotating the blade profile. The center of rotation was the pressure side trailing edge circle attachment point. For the Baseline cascade, $t / s=0.3530$, and this ratio was kept constant as the solidity was increased and decreased by ten percent. However, because of the suction side curvature of the blade, accurate determination of the throat to spacing ratio by hand was difficult. Also, rotating the blade changed 
the axial chord, which in turn affected the solidity. Therefore all of the initial experimental design was done at GEAE in Cincinatti using their blade design computer programs. Using one of these programs, called CUTSL, changes in the blade spacing and orientation were input, and the program calculated the new blade parameters such as throat size and solidity. An iterative process using CUTSL was therefore used to establish the new blade spacings and orientations for the required ten percent solidity changes. Ten percent changes were selected as likely to cause changes in blade loss large enough to be discerned given the estimated range of experimental uncertainty. Note that cascades with five percent solidity changes were also designed but analysis with NOVAK2D resulted in the decision to discard them because the loading changes were too small.

The iterative design method used was as follows:

- Guess a new number of blades in the row (try to match desired pitch).

- Guess a rotation about the trailing edge.

- CUTSL outputs calculated solidity.

- Adjust rotation to match required solidity. Repeat until matched.

- If $t / s$ not correct, repeat above steps until correct.

The method is complicated by the fact that there are many combinations of blade spacing and rotation that give the desired value of solidity, but only one combination also gives the correct throat to spacing ratio. The process was made easier by choosing rotation values well to either side of the expected correct rotation for a given spacing, assuming linearity, and calculating the sensitivity of the solidity to a change in rotation. This could then be used to reach an acceptable solidity value more quickly. Usually five or six guesses for the number of blades sufficed to match both the solidity and throat to spacing ratio, with three iterations of rotation for each spacing (small because of the linearity assumption). The final design for the cascades is shown in Figure 7, which shows the relative blade spacing for the three cascades, and Figure 6, which shows the relative rotations. The geometry is tabulated in Table 1 . The $t / s$ ratios are identical to three significant figures, the rotations are on the order of one degree, and the axial chord changes less than one 
millimeter. The blade spacing and throat were changed by a little less than ten percent to accomplish the ten percent solidity change. The inlet metal angle $\beta_{m 1}$ was assumed constant because of the small curvature of the blade nose, and the exit metal angle $\beta_{m 2}$ was estimated by applying the blade rotation to the Baseline exit metal angle for each case. The unguided turning could not be calculated using CUTSL because of a program bug, so it was estimated with the help of CADAM, a computer-aided-design package. As shown in the table the trailing edge geometry of the blade did not vary for the different solidities.

\subsection{Blade Loading}

The blade loading is represented by the Zweifel loading coefficient $\Psi_{z}$, which is listed in Table 1 . The calculation of the loading parameter caused some difficulty in this experiment because the actual air angles for the cascades could not be measured. The measurement of flow angle in a transonic environment is very difficult, and VPI has no probe capable of such measurements at this time. Consequently, the Zweifel coefficient was calculated using the computed average air angles obtained with NOVAK2D. The inviscid calculations are described in the next chapter, but the values of inlet and exit air angles are listed in Table 2. Plugging into the Zweifel formula given in Chapter Two results in the numbers listed in Table 1. Note that when the solidity is increased from the Baseline case by ten percent, the loading decreases by about ten percent. However, when the solidity is decreased ten percent, the loading increases by about fifteen percent. This difference in the sensitivity of loading to solidity is unexplained. Note that loading is inversely proportional to solidity.

It is important to note at this point that the blade being tested is a derivative of an actual rotor blade for a large commercial aircraft engine. It is different in two respects: scale and forward loading. 
The blade which was tested, called the VPI blade, has an axial chord of $38.1 \mathrm{~mm}$. The actual blade chord is about seventy percent smaller. The scaling was done so that ten blade passages at the Baseline solidity would fit into the available windtunnel section height. This should not affect the results of any loss testing. The front end of the blade was unloaded because the windtunnel can only be used for blades with an axial inlet. The difference in inlet angle between the engine blade and the VPI blade is illustrated in Figure 8. The following figure, Figure 9, shows the effect that the change in geometry has on the loading of the front end of the blade. Note that the suction side Mach number downstream of the throat is identical for the two blades, and that the pressure side Mach number is nearly identical for the aft half of the blade. However, the leading edge loading is much smaller for the VPI blade.

A major concern, then, is whether the conclusions about optimum solidity made for the VPI blade are valid for the engine configuration. The author asserts that the conclusions are valid, given two considerations: separation and boundary layer growth. Separation of flow from the blade surface is usually triggered by excess diffusion, or deceleration, of the flow, and causes an increase in the cascade loss. From Figure 9 it is clear that the leading edge pressure side diffusion is greatest for the VPI blade, and the suction side trailing edge diffusion is identical for the two blades. Thus since separation doesn't occur for any of the solidity cases tested using the VPI profile, it should not occur on the engine profile. The second consideration is the boundary layer growth. Since the pressure side boundary layer is insignificant compared to the suction side (verified by viscous calculations on the VPI blade at GEAE), its effect can be disregarded. Also, the suction side boundary layer growth is dominated by the interaction of the reflected shock from the adjacent pressure side with the suction side boundary layer, downstream of the throat. Since this region is identical for both blades, the effect should be very nearly the same, regardless of the added upstream suction side surface on the engine blade. 


\subsection{Cascade Construction}

New cascade endwalls had to be designed to conduct this experiment, since the blade locations were changed. The most significant change was the number of blades in the cascade. The Baseline number of blades, eleven, was lowered to ten for the solidity decrease and raised to twelve for the solidity increase (see Table 1). Note that these nice even numbers, when multiplied by the respective blade spacings, do not add up to the available tunnel height $(373.4 \mathrm{~cm})$. For this reason the top and bottom blocks of the cascade had to be moved inwards, causing sudden steps in the inlet flowpath. This was judged to have little if any effect upon the tunnel periodicity. Also, because of the changes in blade axial chord, the sidewall static pressure tap locations had to be changed. Drawings of the three cascades, as tested, are found in Figure 10, Figure 11, and Figure 12. The endwalls, which are made of plexiglass and support the entire cascade aerodynamic load, were made on a numerically controlled milling machine. The outer dimensions of an endwall are shown in Figure 13 for reference since they have not been published previously. The numerical control machine allows precise positioning of the blades in the cascade, which is of course very important to this investigation. 


\subsection{Computed Results}

The experiment, as described in the previous chapter, was expected to successfully show the effect of changing the blade solidity. However, as an extra assurance, the blade flow was calculated using NOVAK2D (see Chapter 4) to see the relative differences between the blade Mach number distributions, shock strengths, and base pressures. This chapter will discuss the computed results and how they relate to the experimental design.

Before discussing the results, however, some nomenclature must be explained. Output from NOVAK2D lists exit properties (Mach number, exit flow angle) at the blade exit plane, subscripted (0xit . However, for the experimental results discussed in the next chapter, exit properties are calculated at two stations downstream of the blade exit plane. These are called Station 1 and Station 2 , and are located at axial distance to chord ratios of 1.17 and 1.67 respectively. To facilitate comparison between calculation and experiment, the locations of static pressure taps at Stations 1 and 2 were superimposed on the calculated pressure contours for each blade. By averaging the static pressures (obtained from the pressure contours) at all the tap locations for Station 1, the downstream static pressure $P_{2}$ was computed. Then using the upstream total pressure $\left(P_{t 1}\right)$ the isentropic Mach number, $M_{2 i s}$, is calculated. The pressure ratio $P_{t 1} / P_{2}$ and isentropic Mach number $M_{2 i s}$ are used to compare the calculations with the experimental results. More will be said about this in the chapters on experimental method and results. 


\subsection{Blade Surface Mach Number}

The blade Mach number profiles, obtained from the post-processor for NOVAK2D, are compared on Figure 14. The inviscid calculations were only made for one set of boundary conditions, namely $P_{t 1}=469 \mathrm{kpa}$ at the inlet to the calculation region and $P_{b a c k}=177 \mathrm{kpa}$ at the exit of the calculation region (not the blade exit). This gave a pressure ratio $P_{t 1} / P_{b a c k}$ of 2.65 . Note that the ratio $P_{t 1} / P_{2}$ as estimated from the pressure contours was slightly different. The design pressure ratio for these blades is 2.4 , but the larger value was used since it is near the largest value capable of being tested in the windtunnel for these blades. Because the VPI blades behave very similarly over a wide range of pressure ratios, the large pressure ratio was used to maximize the differences. Although the computational boundary conditions were identical for the three cascades, the resultant values of $M_{2 i s}$ vary slightly because of the calculation method described in the previous paragraph. The isentropic Mach number at Station 1 is about 1.30 for the prescribed boundary conditions. Design Mach number is roughly 1.2 .

Immediately obvious from Figure 14 is the loading differences between the three cascades. The pressure side Mach number clearly shows the increase in loading as solidity decreases, the Baseline solidity falling squarely in the middle. The same trend is also seen on the aft section of the suction side. Overall there was a considerable change in the total loading (enclosed area) with ten percent solidity changes. This was judged to be enough for a good experiment. It is also seen that the diffusion on the forward portion of the pressure side is nearly identical for the three cascades. Since the Baseline cascade shows no leading edge separation, it is therefore unlikely that the solidity changes will cause any unexpected pressure side leading edge separations, which would invalidate 
the results. This is not surprising, since leading edge separation is most likely to be caused by a change in flow incidence, which is very small for the blade rotations applied. Note that the pressure side exit Mach number is 1.0 for all three cascades, since the throat is located at the beginning of the trailing edge circle.

On the suction side, three features are of interest. First, the rise and sudden dip in the Mach number at $\mathrm{x} / \mathrm{c}$ of about 0.8 indicates the impingement of an oblique shock originating from the pressure side of the adjacent blade. The shock interacts with the boundary layer and likely causes a small separation bubble. Re-attachment of the boundary layer causes another oblique shock, which looks like a reflection of the impinging shock. More information on this can be found in Reference 7. For the Baseline case, downstream of the shock interaction the flow continues to accelerate until a small diffusion near the trailing edge. Flow diffusion at a blade surface is an established source of increased loss, and is generally to be avoided as much as possible. However, for the low solidity case (UN10), the magnitude of the trailing edge diffusion is almost doubled, and for the high solidity case (OV10) the flow accelerates up to the trailing edge. These differences are a consequence of using the same blade profile at different spacings with no shape optimization. They have the potential for complicating the results, making it difficult to discern the effect of solidity change. Finally, the suction side exit Mach number differs between the three cases. In fact, it increases from 1.32 to 1.4 as the solidity increases. Since the unguided turning decreases as the solidity increases, it seems more likely that the suction side exit Mach number would decrease (unguided turning is a measure of expansion downstream of the throat). However, because the throat to spacing ratio was kept constant, this did not occur. Note that the average exit Mach number $M_{\text {exit }}$ is nearly identical for the three cascades, even though the suction side exit Mach number differs (see Table 2). 


\subsection{Inlet and Exit Flow}

NOVAK2D outputs the pitch averaged inlet and exit plane Mach number and flow angles. The values are listed in Table 2. From the values of $M_{\text {exit }}$ and $\beta_{2}$ it seems that the design goals have been met. The average exit angle varies by a degree or less, and the exit plane average Mach numbers are nearly identical. The inlet Mach number and flow angle are unchanged at 0.21 and $0^{\circ}$, respectively. From the inlet and exit Mach numbers, it is seen that the reaction is nearly unchanged between the three solidity cases, with an identical value to two significant figures of 0.97 for all cases. Thus reaction will not complicate the results.

The calculated blade surface static pressure for the three cascades is plotted for reference in Figure 15. This plot should be used for comparison with experimental results since the experimental isentropic Mach number should not be compared to the actual Mach number plotted in Figure 14, which is calculated from the actual total pressure through the blade passage.

\subsection{Convergence}

Also listed in Table 2 is convergence information. The convergence factors EMAX and EAVE for all three cases are well below the accepted level described in Chapter Four. The number of calculation nodes (i.e., triangles), however, is about fifty percent smaller for the Baseline case. This is an indication of the amount of grid refinement, which is less for the Baseline case. The pressure contours, explained in the next section, show this difference. 


\subsection{Flowfield Pressure Contours}

The pressure contours obtained from the post-processor for NOVAK2D are shown in Figure 16 through Figure 18. The sonic line, which is given by $P=36 \mathrm{psi}$, is seen to originate on the pressure side just upstream of the trailing edge circle and end upstream on the suction side. It is not affected much by the change in solidity, except that the sonic point on the suction side moves slightly upstream for the low solidity case (UN10, Figure 16). The suction side shock is seen clearly in each case, propogating downstream at a slight angle from axial. The pressure side shock is not so obvious but can be discerned by the peaks in the pressure contours. However, because of the inviscid nature of the calculations, no interaction arises between the pressure side shock and the suction side flow when the shock impinges on the suction side of the adjacent blade.

As mentioned in the above section, the amount of grid refinement for the Baseline cascade is less than for the others. This is clear from the packing of the pressure contours at the shock, which is denser for the high and low solidity cases. This difference in packing does not affect the predicted overall pressure change across the shock, just the spatial distribution of that change. Since the prediction is inviscid, the spatial distribution of the pressure change is not correct for any resolution anyway. An indication of the shock strength can be found by simply noting the amount of pressure rise across the closely packed contours. This method reveals that the suction side shock strength increases and the pressure side shock strength decreases as the solidity is decreased. Thus, a solidity decrease appears to shift the trailing edge expansion around the trailing edge toward the suction side. Specific shock strengths will be given in the chapter on experimental results, where they will be compared with the experimental values. Also by looking at the pressure immediately behind the trailing edge, the calculated base pressure can be found. This is more easily done when the trailing edge region is magnified with the post-processor. Doing so reveals that the base pressure increases as the solidity decreases. Again, more will be said about this in comparison with the experimental results. 


\subsection{Overview of Experimental Method}

In this chapter a brief overview of the testing facility, data collection techniques, and data reduction routine will be given. No emphasis will be placed on the details, which can be found in several previous theses by students who have worked on this project. In particular, see Bertsch (Ref. 4) for a detailed discussion of the experimental method.

\subsection{The Facility}

The testing facility is a transonic blow-down windtunnel. The air supply is pressurized by a four-stage Ingersoll Rand compressor and stored in tanks until blow-down. The maximum tank pressure used for transonic tests is about $1725 \mathrm{kpa} \mathrm{(250} \mathrm{psi).} \mathrm{Tunnel} \mathrm{run} \mathrm{time} \mathrm{is} \mathrm{about} 18$ seconds, during which time the upstream total pressure is held constant by a control valve with a computerized feedback circuit. Different run Mach numbers are obtained with different initial valve openings. There is also a safety valve upstream of the control valve which is used to start and stop the tunnel flow. 
Downstream of the valve there is a $90^{\circ}$ turn, a flow straightener, and a transition section which connects the circular duct to the rectangular test section. The test section has a useful testing area of $15.2 \mathrm{~cm}$ wide by $37.3 \mathrm{~cm}$ high. See Figure 19 for a photo of the test section. The inlet is from the left, and the exit is out the bottom, going into the transition duct at the right which attaches to the outlet pipe and the atmosphere. Attached to the bottom of the test section is the traversing mechanism for the downstream total pressure probe. Through the window in the round aluminum door the removable test section can be seen. The small tank on top of the test section is used for coolant injection, which was not done in this experiment. A view of one of the removable cascades, in this case low solidity (UN10), is seen in Figure 20. There are ten blades, plexiglass endwalls, aluminum blocks at the top and bottom, pins and cap screws to hold the blades to the endwalls, and static pressure taps on the front endwall.

\subsection{Data Collection}

The following is a list of the variables measured during a windtunnel run. They are not in any particular order.

- Atmospheric pressure, $P_{a t m}$.

- Upstream total pressure, $P_{t 1}$, during entire run (transducer "RIESS").

- Total pressure difference, $P_{t 1}-P_{t 2}$ or $\Delta P_{t}$, during entire run (transducer "FLO").

- Endwall static pressures, both stations, $\left.P\right|_{S T N 1}$ and $\left.P\right|_{S T N 2}$, at the beginning of the run.

- Upstream total pressure, $\left.P_{t 1}\right|_{32 c h}$, at the same time as the endwall static pressures.

- Blade static pressures.

- Shadowgraph pictures. 
The atmospheric pressure is obtained from a barometer once during each set of runs. The upstream total pressure $P_{t 1}$ is measured by a stainless steel tube pointed into the flow, mounted in one of the test section doors upstream of the cascade. The tube is connected by plastic tubing to a transducer labeled "RIESS." The data acquisition computer collects data from RIESS throughout the run. The downstream total pressure for the difference $\Delta P_{t}$ is measured by a traversing total pressure probe, which traverses two blade passages during a run. The traverses are done at one of two axial locations, either Station $1(x / c=1.17)$ or Station $2(x / c=1.67)$. The downstream total pressure from the probe is input, along with the upstream total pressure supplied to RIESS, into a differential transducer called "FLO." The signal from FLO is also read by the data acquisition computer at the same time as the data from RIESS is read. About eight hundred data points are taken during a typical run, each data point being a set of RIESS, FLO readings. An example of the data taken by FLO is shown in Figure 52. Left to right on the figure is equivalent to bottom to top in the cascade. That is, data is taken while the traverse travels upwards. The data from RIESS, if shown on the same figure, would be a nearly straight line at a level higher then the $\Delta P_{t}$ peaks measured by FLO.

A separate data acquisition system, referred to as the 32-channel PSI system, records endwall static pressures. The system records these static pressures only once, at the beginning of the run. Thirteen pressures are measured at Station $1\left(\left.P\right|_{S T N 1}\right)$ and thirteen are measured at Station 2( $\left.\left.P\right|_{\text {STN }}\right)$. The distance between ten taps spans one blade spacing, so the endwall taps are spaced differently for each cascade. The PSI system also measures the upstream total pressure $\left.P_{t 1}\right|_{32 c h}$ using the same probe as RIESS, but only records the value at the beginning of the run. The pressures measured by the PSI system are used to calculate the isentropic Mach number for the run, as described below, and also as an indication of shock strength and periodicity. An example of the data from the PSI system is shown in Figure 40.

The blade static pressures are measured with taps imbedded in the blade body that are open at the blade surface. The locations of these taps is given in Figure 39. Note that the axial location of the taps differs between the three cascades because of the blade rotations. The pressures from the taps 
are measured by the PSI system. Finally, shadowgraph pictures are taken for some runs. An example is given by Figure 74 .

\subsection{Assumptions}

The major assumptions made during the testing and data reduction are listed below:

- Total temperature is constant through the blade row for the entire run at a value of 283 degrees Kelvin.

- The endwall static pressures measured by the PSI system are the same as the static pressures at the cascade center.

- The traversing probe travels at a constant rate of speed throughout the run.

- The static pressure behind the bow shock on the traversing probe is the same as the measured endwall static pressure.

- The bow shock on the traversing total pressure probe is a normal shock.

- The blade passage flow is assumed isentropic for calculation of the exit Mach number $M_{2 i s}$.

- The Mach number in front of the traversing probe bow shock is assumed to be equal to $M_{2 i s}$.

- The cascade flow is assumed to be air at standard conditions. 


\subsection{Data Reduction}

Data reduction is done with a Fortran computer program from the data described above. The aim of the data reduction is to calculate the mass-averaged total pressure loss for the cascade, which is given by

$L=\frac{\int_{0}^{1} \rho V\left(\frac{P_{t 1}-P_{t 2}}{P_{t 1}}\right) d y}{\int_{0}^{1} \rho V d y}$

where $0 \leq y \leq 1$ represents two blade spacings. The reduction process is outlined below.

- Endwall static pressures for each station are averaged over one blade spacing to get $\left.\overline{P_{2}}\right|_{S T N 1}$ and $\left.\overline{P_{2}}\right|_{S T N 2}$.

- At Station $1, M_{2 i s}$ is calculated from $\left.P_{t 1}\right|_{32 c h}$ and $\left.\overline{P_{2}}\right|_{S T N 1}$.

- $\quad M_{2 i s}$ is then correlated as a function of $P_{t 1}$ (see Figure 21).

- At Station 2, $\left.\overline{P_{2}}\right|_{S T N 2}$ is correlated as a function of $P_{t 1}$ (see Figure 22).

The next six steps are done for each data point:

- $\quad$ Read in RIESS, FLO data points.

- Compute static pressure $P$ from $P_{t 1}$ and $M_{2 i s}$.

- If the Mach number at the traverse probe is greater than one, then compute the actual $P_{t 2}$ behind the bow shock by iteration. 
- Compute static temperature $T$ from $T_{t 1}$ and $M_{2 i s}$ by the isentropic relation.

- Compute density $\rho$ from static temperature and pressure with the ideal gas law.

- Compute velocity $V$ in exit flow direction from isentropic Mach number and temperature using the definition of Mach number.

- Obtain $M_{2 i s}$ from the Mach number correlation.

Finally, the loss is calculated and the results averaged:

- Integrate by the trapezoid rule over two blade passages to obtain the mass averaged loss $L$.

- Divide by 2 to average the loss for one passage.

- Average $M_{2 i s}$ values over a passage.

An example of the loss calculated in this way plotted versus the average exit isentropic Mach number is shown in Figure 60. 


\subsection{Experimental Results}

In this chapter the experimental data will be examined in detail. The aim will be to justify the validity of the loss data presented at the end of the chapter. Also, the experimental results will be compared with the NOVAK2D computations where possible, to give an idea of the usefulness of the code as a design tool.

\subsection{Blade Surface Static Pressures}

The blade surface static pressure distributions are compared to the computed distributions in Figure 23 through Figure 25. The calculated pressure ratio $P_{t 1} \mid P_{2}$ for all three solidity cases was about 2.7 , and was obtained by averaging the static pressure from the pressure contours at Station 1 tap locations. Although the pressure ratio was not matched exactly in the experiments, values on both sides of the calculated value are plotted for comparison. For the predicted distribution note the two dips in static pressure at the trailing edge. These are caused by the inviscid assumption and are not real. Also, the inviscid base pressure can be read from the distribution by noting the high 
point between the dips. The experimental data are plotted as discrete points which are connected. The actual pressure distribution may not be represented by the connecting lines, especially in the region of shock/boundary layer interaction. The trailing edge taps appear as single points, since connecting them to the other surface taps makes no physical sense. These taps give the experimental value of base pressure.

On the pressure side of the blade, the experiment and computations agree well. However on the suction side, the loading is under-predicted downstream of the throat. Also the impingement of the pressure side shock on the suction side is predicted too far upstream on the blade. This is reflected in the pressure side shock location on the pressure contours. For example, see Figure 70 for a comparison of the actual shock pattern to the predicted pattern. The pressure side shock is predicted too far upstream. Apparently the pressure drop across the shock impingement is predicted fairly well, but the wide spacing of the taps may leave out important information. Finally, it appears that the exit static pressure is predicted well even though the loading is under predicted.

\subsection{Comparison of Blade Loadings}

Important to the successful interpretation of the loss results is an understanding of how similar the flowfields are for the three solidity cases. Comparisons of the blade loadings for different exit isentropic Mach numbers are found in Figure 26 through Figure 32. The pressure side loading and acceleration differences are consistent for the entire range of Mach numbers tested. This consistency implies that there are no separations or other irregularities in the pressure side flow caused by the solidity changes. Also, the degree of acceleration on the suction side up to an $\mathrm{x} / \mathrm{c}$ value of about 0.65 is the same for all Mach numbers. This supports the assumption that the loss results obtained in this experiment can be extended to the fully-loaded engine blade, since the only 
differences in the blade profiles occur for $\mathrm{x} / \mathrm{c}$ less than about 0.4 . Note that the trends in suction side peak and exit Mach number in Figure 26 are not repeated for the other Mach numbers. There is no experimental consistency in the order of suction side peak and exit Mach numbers. However, the base Mach numbers, which are given by the single points on the figures, show a consistent trend. The base Mach number, while not physical, can be used to back out the base pressure. The important fact is, from the information on the figures, the base pressure is always greatest for the low solidity case at a given Mach number. This will be discussed in greater detail in another section.

The most important piece of information to get from the loading comparisons is the suction surface diffusion. Diffusion is the common term in the industry for a deceleration on the blade surface. In Figure 26 it is clear that the high solidity case (OV10) has a larger suction surface diffusion than the other cases. Specifically, for $x / c$ values between 0.62 and 0.76 , OV10 exhibits a larger diffusion. Increased suction surface diffusion translates directly into increased profile loss. This is clear from past blade design experience in the industry. Thus, OV10 is expected to have a higher loss than the lower solidities, independent of the change in solidity. This is a complicating factor. The OV10 cascade continues to show this larger diffusion for Mach numbers below 1.19, while the UN10 and Baseline cases show less diffusion and in some cases even acceleration up to the trailing edge.

Fortunately suction surface diffusion ceases to be a problem for $M_{2 i s}>1.19$. Referring to Figure 30, all three solidities show roughly equal diffusions following the shock/boundary layer interaction followed by similar accelarations up to the trailing edge. Since $M_{2 i s}=1.19$ is near the design point for these blades, this is encouraging. The solidity results for Mach numbers near and above the design point should not be complicated by differing diffusions.

The blade loadings for the whole range of tested Mach numbers are overlaid in Figure 33 to Figure 38. Separate plots are shown for each blade. Also, both the static pressure distributions and isentropic Mach number distributions are shown for comparison, noting that the isentropic assumption implies constant total pressure through the blade passage. The Mach number profile 
shapes are nearly the same as the static pressure profiles, so the isentropic assumption is reasonable for the purpose of comparing blade loading.

\subsection{Wall Static Pressure}

The wall static pressure data shown in Figure 40 through Figure 45 was obtained using the 32-channel PSI system. Left to right on the horizontal axis corresponds to top to bottom on the wall taps at each station. The spacing between taps is normalized to the blade pitch for each cascade so the different solidities can be compared. Height to spacing ratios of 0.0 and 1.0 represent taps located directly behind blade centerlines. The two blades on either side of the instrumented passage are also the two which are traversed with the total pressure probe. Note that the Baseline cascade has the taps located differently from the other cascades, so that only one blade centerline is included (at 0.0 ). Also, values less than zero or greater than one on the horizontal axis indicate taps outside of the instrumented passage. The cascade periodicity can be judged by observing static pressure values separated by a height/spacing value of 1.0. For example, on Figure 40 , the first three static pressures on the left should match the last three on the right. By looking at all the figures in this way, it is seen that the periodicity is fair.

The computed wall static pressures are shown on the figures with the measured values. The computed values were obtained by overlaying the tap locations on the calculated pressure contours and reading the values. This method is admittedly rough but should give an indication of the accuracy of the calculations. The match between computed and experimental results is good, but the computations over-predict the amount of pressure rise across the suction side shocks. For example, on Figure 43 (Baseline cascade) the shock passes through the taps at horizontal axis values between -0.4 and -0.2 . The predicted pressure rise is larger than the measured value. For 
the UN10 and OV10 cascades, the shock passes through the taps at horizontal axis values between 0.6 and 0.8 .

The wall static pressure results support the assumption that the static pressure is nearly constant across the cascade span. The measured values are taken at the wall, while the computed values are at the cascade pitchline. In several instances the static pressures match very well, especially at Station 1. One notable exception is shown in Figure 41 for the UN10 cascade. However, looking at the pressure contours for this cascade, it appears that the suction side shock has been refined too much, bunching the pressure rise in too small of a region. This is also complicated by the fact that Station 2 is located very near the aft calculation boundary condition, which is a constant static pressure. These two factors account for the different shape of the calulated static pressure survey given in Figure 41.

\subsection{Suction Side Shock}

The static pressure rise across the suction side shock downstream of the cascade exit is plotted in Figure 46 and Figure 47. While there is some arbitrariness in choosing how far away from the shock the static pressures are picked to form the ratio, the figures still give a general picture of the shock behavior. From the figures it is clear that a penalty for decreased solidity is an increased suction side shock strength. The calculations also showed that a decrease in solidity reduced the pressure side shock strength. Thus a reduction in solidity shifts the trailing edge expansion around toward the suction side. The calculated results for the suction side shock, one Mach number only, are shown on the figures. While the predicted strengths are about fifteen percent too big, the trend is correct. Note also that the shock decreases in strength by about ten percent for all three solidites between Station 1 and Station 2. The increase in suction side shock strength with decreased solidity 
is bad news for this particular blade. An efficiency penalty in the low pressure turbine for the engine has been traced to the effect of the passing suction side shock from the high pressure rotor which the VPI blade is based on. A decrease in solidity will aggravate this problem.

An attempt was made to characterize the effect of solidity on the angle of the suction side shock. The results are shown in Figure 48. There is a large amount of scatter in the data, due to the fact that the shocks are not steady. The unsteadiness has been verified by high-speed films of the cascade flow. The shock angle varies by as much as thirty degrees at a high frequency, thus the angle captured by the shadowgraphs does not mean much. The angle plotted on the vertical axis was measured from the vertical (tangential) direction in a clockwise fashion, from shadowgraph pictures. While no trend was established between the solidity cases, it is seen that the shock angle does increase with Mach number, as expected.

\subsection{Base Pressure and Unguided Turning}

The blade base pressure has been shown to have a significant effect on the aerodynamic loss of blade rows. In particular, Sieverding (Ref. 32) has correlated the effect, as seen in Figure 51 . The base pressure results for this experiment, plotted on the same axes as the correlation, appear in Figure 49. Sieverding correlated the base pressure as a function of the suction side unguided turning ( $\varepsilon$ in his notation) and the trailing edge wedge angle $(\delta)$. The values of the correlating parameter $(\varepsilon+\delta) / 2$ for the tested cascades are listed below: 


\begin{tabular}{|ccc|}
\hline Cascade & $\boldsymbol{\sigma}$ & $\frac{\boldsymbol{\varepsilon}+\boldsymbol{\delta}}{2}$ \\
\hline UN10 & 0.923 & 8 \\
Baseline & 1.025 & 9 \\
OV10 & 1.128 & 12.4 \\
\hline
\end{tabular}

By comparison with the correlation, it is seen that the measured base pressures are slightly higher than predicted. The hump shape of the correlation through regions $b$ and $c$ is clearly shown by the OV10 cascade. Note that the trailing edge wedge angle $\delta$ is constant for the three tested cascades. It is generally accepted (see References 32 and 37) that an increase in base pressure corresponds to a decreased profile loss. Thus from Figure 49, a loss prediction based solely on base pressure would predict that loss decreases with decreased solidity.

This picture is complicated by the suction side unguided turning. For this experiment UGT increases as solidity is decreased. In general, a low value of UGT is desired to limit the amount of expansion downstream of the blade throat. For large expansions on highly loaded blades, the suction side exit Mach number is significantly greater than one, which is usually the pressure side exit Mach number. This difference in exit speeds causes a lot of trailing edge expansion on the suction side, resulting in a high suction side shock strength and increased loss. The increased shock strength is verified by the results of the previous section. However, for blades of this type it has been established (Ref. 10) that very low values of UGT cause increased losses at low pressure ratios, when the blade suction side velocity becomes super-critical. In particular, for low values of UGT the suction side throat is too far aft on the blade, resulting in a rapid diffusion at the throat for low pressure ratios labelled the "transonic hole". This effect is clear in Figure 38 for the OV10 cascade, which has the lowest value of UGT. Note that small values of UGT cause increased losses only at low values of pressure ratio, while at the design point the low UGT does not seem to affect the 
loss. Thus there must be a trade-off between shock strength and transonic hole effects caused by the UGT. This compromise will be discussed in the context of the pressure loss results.

The experimental base pressures for $M_{2 i s}=1.30$ are compared to the values obtained from NOVAK2D in Figure 50. Note that predicted values are shown for five different solidities. The initial experimental design called for five percent changes in solidity as well as the final ten percent changes. These were ruled out, but the base pressures are plotted here. The predicted base pressures are too large, certainly because of the absence of viscous effects in the computation. This graph again points out that base pressure increases as solidity is decreased.

\subsection{Traverse Data}

Representative data obtained by the traversing total pressure probe is given in Figure 52 through Figure 59. The data for all three solidities is overlaid in each case, and separate plots are made for each probe station. Left to right on the horizontal axis corresponds to probe movement from bottom to top in the cascade test section. From these plots the differences in the cascade blade pitches are obvious. OV10 has the smallest pitch, UN10 the largest. The spikes in the data at the far right of the plots should be ignored, as these are transients caused by shutting the tunnel control valve at the end of the run. The vertical axis is $\Delta P_{t}=P_{t 1}-P_{n}$.

Some idea of the evolution of losses is given by these plots. For $M_{2 i s}=0.76$, there are only wake losses. Some loss due to normal shocks is evident at $M_{2 i s}=1.00$, and large losses due to oblique shocks appear in the plots for $M_{2 i s}=1.22$. Finally, at $M_{2 i s}=1.35$ the oblique shock losses are on the same order as the wake losses. Note that the pattern of pressure drop through the oblique 
shocks is very similar for all three solidity cases. The relative levels of total pressure drop between the solidity cases corraborates the loss data which is presented in the next section. From the traverse data, it appears that loss decreases as the solidity is decreased.

Two aberrations among the traverse plots are worthy of discussion. First, for Figure 52, the order of wake peak heights is reversed from the normal trend. Also, the wake width is larger for higher solidity. On all the other plots, the wake widths are usually nearly identical. This is unexplained. Secondly, for Figure 55, there is no distinction between wake and shock losses for the OV10 cascade. By referring to the shadowgraph picture for this run, Figure 91, it is seen that strong oblique shocks are present over a large portion of the downstream flow. This is not the case for the other cascades and explains the shape of the traverse plot. A very large integrated loss value will result from this situation. Such behavior has been shown by other cascades in the transonic tunnel at high Mach numbers, and is suspected to be caused by strong shock reflections from the downstream shear layer. The loss data from this run is therefore not included on Figure 60.

\subsection{Loss Results}

The mass averaged total pressure loss is plotted against average isentropic Mach number for all three solidities in Figure 60 and Figure 61, by traverse station. Uncertainty bars for the Baseline cascade are placed on Figure 60 for reference; the uncertainty calculation is outlined in the next chapter. It is clear that the UN10 cascade $(\sigma=0.923)$ has the same loss as the Baseline cascade $(\sigma=1.025)$ within experimental uncertainty. This is true at both Station 1 and Station 2. One note of caution about these loss plots: the loss value at the highest Mach number for each cascade should be treated with suspicion, a consequence of the Mach number versus total pressure correlation used in the data reduction. The ends of a correlation curve are always dubious. Note 
also that there is an uncertainty in the isentropic Mach number, which is 0.03 for low Mach numbers and 0.04 for high Mach numbers.

An interesting feature of the loss curves is the unusual shape of the curve for the OV10 cascade. At low Mach numbers, there is a large gap in the loss between OV10 and the other cascades which narrows for higher Mach numbers. This is attributed to the transonic hole effect discussed in the section on base pressure and unguided turning. The OV10 blade has a lower value of UGT than the other blades, which causes an increased loss at low pressure ratios. This effect diminishes for higher pressure ratios, as reflected on the loss curve. This conclusion is verified by the Mach number distributions for the OV10 blade (Figure 38), which show a large level of suction side diffusion for low pressure ratios. The above information, coupled with the fact that suction side shock strength increases for the UN10 blade, leads to the conclusion that the value of UGT for the Baseline blade $\left(9.6^{\circ}\right)$ is in some sense an optimum. The decrease in loss at large Mach numbers shown by the OV10 cascade cannot be accounted for with the available data.

The raw data was also integrated for the wake regions (peaks on the traverse plots) only, to give an indication of the amount of loss occuring in the wakes. These results are shown on Figure 62. Note that the wake loss denotes both the area on the traverse plots within the wake peaks as well as below them. For exit Mach numbers less than 0.9 , the wake loss then represents only blade boundary layer losses and trailing edge mixing losses. For supercritical velocities the wake losses include some losses due to normal shocks, and for higher Mach numbers some loss due to the oblique shocks. For $M_{2 i s}<0.9$ the wake losses match the total losses. Above that, supercritical velocity is reached and shock losses begin to increase. The wake loss continues to rise as Mach number increases, but less than the total loss. Surprisingly the wake loss begins to decrease for $M_{2 i s}>1.2$. Perhaps this is caused by the shock/boundary layer interaction on the blade suction side moving aft. Since this interaction causes sudden growth in the suction side boundary layer, a smaller exit boundary layer would be the result of a shorter distance between the interaction and the trailing edge. 
In the figures following Figure 62 the uncorrected total pressure loss is shown, as well as a comparison for each cascade between Station 1 and Station 2. Uncorrected loss means there was no compensation for the bow shock on the total pressure probe. Note that the Station 2 loss is always higher than the Station 1 loss, since fully mixed flow is not achieved at Station 1. Finally, the current Baseline cascade loss results are compared to previous data for validation. See Figure 68. While there are differences, they are within the experimental error for the most part. This plot also justifies the claim that the experimental uncertainty is very small for low Mach numbers and increases with Mach number.

\subsection{Pressure Contours vs. Shadowgraphs}

The pressure contours predicted by NOVAK2D are compared to shadowgraph pictures in Figure 70 and following figures. Although the Mach numbers for the contours and shadowgraphs are not matched exactly, the exit isentropic Mach number is about 1.30. Overall, the flow match is good, especially for the suction side shock near the trailing edge. Farther from the trailing edge, however, the calculated results are influenced by the constant pressure boundary condition, the shadowgraphs show shock reflections from the downstream free shear layer, and the match is poor. However, the pressure contours show a pressure disturbance downstream of the cascade exit plane which mimics the shear layer shock reflections shown on the shadowgraphs. Given the difference in downstream boundary conditions, the pressure contours and shadowgraphs cannot be expected to match well far from the trailing edge.

For all three solidities, the pressure side shock is predicted too far upstream. This is assumed to be a consequence of the inviscid assumption. The angle of the pressure side shock is also incorrect in 
the predictions. For the OV10 cascade, the trailing edge shock pattern is displaced too far from the trailing edge. Possibly this is due to the combined effect of a large trailing edge blockage and the inviscid assumption.

\subsection{Shadowgraphs}

Shadowgraphs for each solidity case over a range of Mach numbers are presented at the end of the appendix. The aim of including them is to verify the similarity of flow for the three solidity cases, especially the shock patterns. In several instances there are optical flaws which look like flow separation from the blade, but no irregularities on the experimental Mach number profiles point to separation problems. Note also that the shadowgraphs shown were taken near the top of the cascade, were there is a significant amount of shock reflection from the downstream free shear layer. The blade pitches which were traversed to obtain loss data were near the bottom of the cascade and thus located farther upstream from the free shear layer. Shadowgraphs taken at the bottom of the cascade showed less shock reflection from the shear layer. However, the wall static pressure taps at the bottom of the cascade clutter the shadowgraphs taken there.

\subsection{Reynolds Number}

The Reynolds number for a cascade of blades, based on axial chord and upstream flow conditions, is defined as 
$R e=\frac{\rho V c}{\mu}$

Using the definition of mass flow, this can be expressed as

$R e=\frac{\dot{m} c}{\mu A}$

where $\mathrm{A}$ is the flow area. Expanding the mass flow using the ideal gas law and isentropic flow relations,

$\dot{m}=\rho V A=P_{t 1} A \sqrt{\frac{\gamma}{R T_{t 1}}} \frac{M}{\left[1+\frac{(\gamma-1)}{2} M^{2}\right]^{\left[\frac{\gamma+1}{2(y-1)}\right]}}$.

Applying this relationship at a blade throat, where $M=1$,

$\dot{m}=P_{t 1} t h \sqrt{\frac{\gamma}{R T_{t 1}}} \frac{1}{\left[1+\frac{(\gamma-1)}{2}\right]^{\left[\frac{\gamma+1}{2(\gamma-1)}\right]}}$.

The flow area $A$ is in this case the flow through one blade passage, which is $h s$, the span multiplied by the spacing. Combining all of the above, the Reynolds number is then

$R e=\frac{P_{t 1} t c}{\mu s} \sqrt{\frac{\gamma}{R T_{t 1}}} \frac{1}{\left[1+\frac{(\gamma-1)}{2}\right]^{\left[\frac{\gamma+1}{2(\gamma-1)}\right]}}$.

Substituting in the values of air properties at standard conditions (in SI units),

$$
\begin{aligned}
& \mu=1.5 \times 10^{-5} \frac{\mathrm{Ns}}{\mathrm{m}^{2}} \\
& \gamma=1.4 \\
& T_{t 1}=273^{\circ} \mathrm{K}
\end{aligned}
$$




$$
R_{\text {air }}=287 \frac{\mathrm{Nm}}{\mathrm{kgK}}
$$

a useful form is obtained:

$R e=163.1\left(\frac{P_{t 1} t c}{s}\right)$.

The following table shows the Reynolds number results for the three cascades used in this experiment over the range of Mach numbers.

\begin{tabular}{|c|ccc|}
\cline { 2 - 4 } \multicolumn{1}{c|}{} & \multicolumn{3}{c|}{$\operatorname{Re} \times 10^{-6}$} \\
\hline$M_{2 i s}$ & $\mathrm{UN10}$ & Baseline & OV 10 \\
\hline 0.75 & 1.02 & 1.04 & 1.05 \\
1.35 & 1.70 & 1.73 & 1.74 \\
\hline
\end{tabular}




\subsection{Uncertainty Analysis}

Prior testing in the windtunnel used for this experiment has not included an analysis of the experimental uncertainty. This is primarily due to the complexity of the data analysis codes. The difficulty arises from the fact that many of the properties used in the calculation of the mass-averaged loss are derived from other properties which are measured. For example, density and static temperature are not measured directly, but are calculated from measured static pressure and other variables. The data reduction process is discussed in detail in the thesis by Bertsch (Ref. 4). The indirect determination of flow properties is a problem because of the dependence that arises between the properties used in the calculation of loss. Another good example is provided by the form of the mass averaged loss, which has density and velocity terms in both the numerator and denominator, making each portion of the loss coefficient dependent on the other.

Dependence is a problem because it contradicts the primary requirement of the accepted uncertainty calculation method. The method, attributed to Kline and McClintock (Ref. 22), states that if a result $R$ is a function of $n$ independent variables $x$, then the error in $R$ is given by:

$\delta R=\sqrt{\left[\left(\frac{\partial R}{\partial x_{1}}\right) \delta x_{1}\right]^{2}+\left[\left(\frac{\partial R}{\partial x_{2}}\right) \delta x_{2}\right]^{2}+\left[\left(\frac{\partial R}{\partial x_{2}}\right) \delta x_{2}\right]^{2}+\ldots+\left[\left(\frac{\partial R}{\partial x_{n}}\right) \delta x_{n}\right]^{2}}$. 
This formula was proved to give the best estimate of the actual statistical error using several probability density functions as examples. Ideally, the required derivatives are worked out for the result $R$, the uncertainties $\delta x_{n}$ are determined statistically, and the uncertainty in the result is then known.

For this experiment, however, because of the dependence problems mentioned above, the analytical method is not useful. In fact, it would predict an uncertainty much larger than the correct value. Instead, the inputs to the data reduction program are perturbed, i.e. $x_{n}=x_{n} \pm \Delta x_{n}$, one at a time, and the effect on the result $R$ is recorded. The required derivatives can therefore be estimated. It is important to keep the perturbations $\Delta x_{n}$ on the order of the expected values of $\delta x_{n}$ in case the variation of $R$ with $x_{n}$ is not approximately linear. The derivatives and variable uncertainties are then combined according to Kline and McClintock's formula to give the experimental uncertainty.

The uncertainty for this experiment was calulated in two stages, the first stage analytical and the second approximate as described above. The inputs to the mass-averaged loss calculation are given by

$L=L\left(P_{a t m}, M_{2 t s}, T_{t 1}, P_{t 1}, \Delta P_{t}\right)$

where $\Delta P_{t}=P_{t 1}-P_{12}$, which is measured directly by a total pressure probe. The two-stage approach to uncertainty calculation is made necessary by the fact that the isentropic Mach number is not measured directly, but is input into the loss calculation in the form of a Mach number versus upstream total pressure correlation. For calculations at Station 2 the $M_{2 i s}$ term is replaced by $P_{2}$, which again is input into the loss caculation as a correlation of downstream static pressure versus upstream total pressure. The primary measurements used to develop these correlations are obtained from the average of the wall static taps downstream of the blades and from the total pressure tap upstream of the cascade, all measured with the 32-channel PSI system. Thus uncertainty in Mach number and static pressure are determined from the functional relationships

$M=M\left(P_{t 1}, P_{a t m},\left.\overline{P_{2}}\right|_{S T N 1}\right)$ 
and

$P_{2}=P_{2}\left(P_{a t m},\left.\overline{P_{2}}\right|_{S T N 2}\right)$

and all of the measured variables are accounted for.

The uncertainties in the measured variables, with 95 percent confidence, are as follows:

\begin{tabular}{|cc|}
\hline Variable & Uncertainty \\
\hline$\left.\bar{P}_{2}\right|_{S T N}$ & $\pm 0.009 \mathrm{psi}$ \\
$\left.\bar{P}_{2}\right|_{S T N 2}$ & $\pm 0.009 \mathrm{psi}$ \\
$\left.P_{t 1}\right|_{32 c h}$ & $\pm 0.03 \mathrm{psi}$ \\
$P_{a t m}$ & $\pm 0.15 \mathrm{psi}$ \\
$T_{t}$ & $\pm 100^{\circ} \mathrm{K}$ \\
$\left.P_{t 1}\right|_{R I E S S}$ & $\pm 0.01 \mathrm{Volt}$ \\
& $( \pm 0.036 \mathrm{psi})$ \\
$\Delta P_{\mathrm{t} 1}$ & $\pm 0.01 \mathrm{Volt}$ \\
& $( \pm 0.012 \mathrm{psi})$ \\
\hline
\end{tabular}

The distinction between $\left.P_{t 1}\right|_{32 c h}$ and $\left.P_{t 1}\right|_{\text {RIESS }}$ is necessary because the first is measured once by the 32-channel system and the second continuously during the run by a single transducer named "RIESS." The uncertainty in individual $P_{2}$ measurements is $\pm 0.03 \mathrm{psi}$, which gives the above results for the average of ten measurements. $\left.P_{t 1}\right|_{32 c h}$ has the same uncertainty as individual $P_{2}$ measurements since it is also taken with the 32-channel transducer system. The variation in $P_{a t m}$ is determined based on experience rather than proper statistics, since no records of the fluctuations in atmospheric pressure during testing time were kept. The total temperature variation was also guessed based on minimal measurements made previous to this experiment, but this variable turns 
out to have a negligible effect on the experimental uncertainty. Finally, the errors in $\left.P_{t 1}\right|_{\text {RIESS }}$ and $\Delta P_{t 1}$ represent the typical drift of the transducer amplifiers during the period of data collection. The transducers are re-zeroed if the drift exceeds this amount. Note that a simple statistical test of the repeatability of these transducers was conducted, but the repeatability was determined to be an order of magnitude less than the drift error. When the primary uncertainties listed above are input into Kline and McClintock's formula, the uncertainties in Mach number and downstream static pressure are obtained:

\begin{tabular}{|ccc|}
\hline Mach number & $\delta M_{i s}$ & $\left.\delta P_{2}\right|_{\text {STA2 }}$ \\
\hline 0.74 & 0.03 & 0.15 \\
1.17 & 0.035 & 0.15 \\
1.24 & 0.04 & 0.15 \\
1.35 & 0.04 & 0.15 \\
\hline
\end{tabular}

Note that the uncertainty in downstream static pressure is constant with Mach number. This is because the uncertainty in atmospheric pressure dominates that due to the pressure measurement system. The results for Mach number and downstream static pressure are correlated as functions of upstream total pressure, as mentioned above. This correlation will itself introduce some uncertainty. By performing a multiple regression on a typical correlation curve, the correlation uncertainty is found to be about twenty five percent of the absolute uncertainty in Mach number and static pressure. Therefore the error introduced in the correlation will be neglected.

Now that all of the uncertainties necessary to calculate the loss range are known, the computational method described above is used. The results for derivatives, variable uncertainties, and final corrected loss uncertainty for several Mach numbers at both stations are listed in Table 3 in the appendix. Note that the uncertainty in the corrected loss $L_{c}$ varies widely with Mach number. This agrees well with the scatter observed with the experimental set-up over the last few years, 
exemplified in the plot comparing Baseline loss data taken on different days (Figure 68). Note that, while the uncertainty calculation was done for the Baseline cascade, the result should be the same for the other two cascades tested.

Several useful conclusions can be drawn from this uncertainty analysis. First, several of the errors could be significantly reduced. For example transducer drift is much larger than the random error in transducer readings. A zero circuit could solve this problem. Second, the effect of uncertainty in atmospheric pressure has been discovered. According to past experimental practice, $P_{\text {otm }}$ was measured only once during data collection since the variation was assumed to have little effect on the results. This is apparently not true and the atmospheric pressure should be measured more frequently to lower the uncertainty. Finally, it is seen from Table 3 that the uncertainty in corrected losses is larger for Station 1 than for Station 2. This disparity arises from the fact that Mach number is correlated for Station 1 and static pressure is correlated for Station 2. The calculation of Mach number from the static and total pressures (which are the measured variables for both stations) introduces an uncertainty which is then inherent in the Mach number correlation. The two different correlations are used strictly for traditional reasons, and it is apparent that static pressure should be correlated for both stations to reduce the uncertainty. 


\subsection{Conclusion}

The research goal of determining the effect upon loss of changes in solidity of the VPI blade has been met. The experimental design was simple and effective in determining the effect of solidity. The decision to keep the throat to spacing ratio of the blades constant for different spacings kept the blade reaction constant. Although the suction side unguided turning and diffusion changed for the different solidities, a reasonable conclusion about the effect of solidity change was still possible. No increase in total pressure loss was caused by a ten percent decrease in solidity from the design value. The Zweifel loading coefficient for the decreased solidity cascade $\left(\Psi_{2}=0.83\right)$ was significantly higher than the design practice value for high pressure turbine nozzles $\left(\Psi_{z}=0.5\right.$ to 0.7$)$. The relative change in cascade total pressure loss was found to decrease as solidity was decreased, suggesting that the original design was not at the optimum solidity, but near it. The lowest solidity tested here corresponds to a Zweifel loading coefficient of about 1.25 for the fully loaded, engine configuration blade pitchline profile. This is significantly higher than the design practice value of 1.1 typically used for transonic rotor blades of this type.

The inviscid calculation code NOVAK2D predicted relative changes in flow characteristics between the three solidities well. However the absolute level of these charactersitics was incorrect due to the inviscid assumption. The code was thus useful in predicting the amount of solidity change required to obtain observable changes in loading and other flow characterisitics. 
The next step in this testing program should be a repeat of this experiment with the fully loaded engine configuration blade profiles. A modification of the windtunnel test section to allow inlet flows other than axial would be required. Instead of solidity changes of plus and minus ten percent, only decreases in solidity should be studied since an increase is known to increase loss. Detailed boundary layer studies such as location of transition and suction side trailing edge boundary layer profile measurement should be added to the testing program. Combined with more comprehensive measurements of the rate of mixing loss generation, this test program could provide a helpful design data base for further turbine blades with this level of loading. 


\subsection{Bibliography}

1. "Advanced Small Axial Turbine Technology," Defense Technical Information Center Technical Report, Final Report for Period July 1972 - June 1976, distributed by Defense Logistics Agency, May, 1977.

2. Baines, N.C., Oldfield, M.L.G. and Simons, J.P., "The Aerodynamic Development of a Highly-Loaded Nozzle Guide Vane," ASME paper No. 86-GT-229.

3. Balje, O.E. Turbomachines: A Guide to Design, Selection, and Theory. John Wiley and Sons, New York, 1981, pp. 132-136,141-143.

4. Bertsch, R., "An Experimental Examination of the Influence of Trailing Edge Injection on Blade Losses in Transonic Turbine Cascades," VPI Thesis, 1990.

5. Cherry, D.G. and Thomas, M.W., "Single Stage HP Turbine Rotor Cascade Program Design, Test and Performance Report," General Electric Aircraft Engine Group Technical Information Series No. R75AEG492, March, 1977.

6. Cohen, H., Rogers, G.F.C., and Saravanamuttoo, H.I.H. Gas Turbine Theory. John Wiley and Sons, NY, pp. 206-207.

7. Dietrichs, H.J. and Braunling, W., "Flow Phenomena in Transonic Turbine Cascades: Detailed Experimental and Numerical Investigation," ISABE paper No. 87-7031.

8. Dixon, S.L. Fluid Mechanics, Thermodynamics of Turbomachinery. Pergamon Press, Oxford, 1978.

9. Doughty, R.L., "Transonic Turbine Linear Cascade Studies," General Electric Aircraft Engines Report No. VPI-0890-1 (1990).

10. Dunbar, L.W., " A Review of the Cooling Flow and Aerodynamic Considerations of F101 Type High Pressure Turbine Nozzles, " GEAE Turbine Aerodynamic Design Memorandum, , August 23, 1979.

11. Ewen, J.S., Huber, F.W., Mitchell, J.P., "Investigation of the Aerodynamic Performance of Small axial Turbines," Transactions of the ASME, October 1973, pp. 326-332. 
12. Gostelow, J.P. Cascade Aerodynamics. Pergamon Press, Oxford, 1984, p. 244.

13. Gostelow, J.P., "The Present Role of High Speed Cascade Testing," ASME paper No. 81-GT-95.

14. Holeski, D.E. and Stewart, W.L., "Study of NASA and NACA Single-Stage Axial Flow Turbine Performance as Related to Reynolds Number and Geometry," Transactions of the ASME, July 1964, pp. 296-298.

15. Hauser, C.H. and Plohr, H.W., "Two-Dimensional Cascade Investigation of the Maximum Exit Tangential Velocity Componenet and Other Flow Conditions at the Exit of Several Turbine Blade Designs at Supercritical Pressure Ratios," NACA Research Memorandum, NACA RM E51F12, August 23, 1951.

16. Holmes, D.G. and Connell, S.D., "Solution of the 2D Navier-Stokes Equations on Unstructured Adaptive Grids," General Electric Aircraft Engines Internal Publication.

17. Holmes, D.G. and Connell, S.D., "Quasi-3D Solutions for Transonic, Inviscid Flows by Adaptive Triangulation," General Electric Aircraft Engines Internal Publication.

18. Horlock, J.H. Axial Flow Turbines. Robert E. Krieger Publishing Co., Malabar, Fla.

19. Howell, A.R., "The Aerodynamics of the Gas Turbine," The Journal of the Royal Aeronautical Society, Vol. 52, June 1948, pp. 329-348.

20. Kacker, S.C., and Okapuu, U., "A Mean Line Prediction Method for Axial Flow Turbine Efficiency," ASME paper 81-GT-58 (1981).

21. Kiock, R., Lehthaus, F., Baines, N.C., and Sieverding, C.H., "The Transonic Flow through a Plane Turbine Cascade as Measured in Four European Wind Tunnels," Journal of Engineering for Gas Turbines and Power, Vol. 108, April 1986, pp. 277-284.

22. Kline, S.J. and McClintock, F.A., "Describing Uncertainties in Single Sample Experiments," Mech. Eng., January 1953, p.3.

23. Korakianitis, T.P., "Design of Airfoils and Cascades of Airfoils," AIAA Journal, Vol. 27, No. 4, April 1989, pp. 445-461.

24. Kuofang, Q. and Naixing, C., "New Correlations of the Two-Dimensional Turbine Cascade Aerodynamic Performance," ASME paper No. 81-GT-128.

25. Lemond, W.D., Thomas, M.W., and Walker, N.D., "Stator Annular Cascade Program Design and Test Report," General Electric Aircraft Engine Group Technical Information Series No. R78AEG197, February, 1978.

26. Mee, D.J., Baines, N.C., Oldfield, M.L.G., and Dickens, T.E., "An Examination of the Contributions to Loss on a Transonic Turbine Blade in Cascade," ASME paper No. 90-GT-264.

27. Moustapha, S.H., Okapuu, U., and Williamson, R.G., "Influence of Rotor Blade Aerodynamic Loading on the Performance of a Highly Loaded Turbine Stage," ASME paper No. 86-GT-56.

28. Oldfield, M.L.G., Kiock, R., Holmes, A.T., and Graham, C.G., "Boundary Layer Studies on Highly Loaded Cascades using Heated Thin Films and a Traversing Probe," Journal of Engineering for Power, Vol. 103, January 1981, pp. 237-246. 
29. Plohr, H.W. and Nusbaum, W.J., "An Experimental Cascade Study of the Effects of a Solidity Reduction on the Two-Dimensional Aerodynamic Characteristics of a Turbine-Rotor Blade Suitable for Air Cooling," NACA Research Memorandum, NACA RM E52B27, May 8, 1952.

30. Singer, R.T. Jr., "An Experimental Examination of the Effect of Trailing Edge Injection on the Aerodynamic Performance of Gas Turbine Blades, " VPI Thesis , 1988.

31. Shepherd, D.G. Principles of Turbomachinery. Macmillan Company, New York, 1956, pp. 195-199.

32. Sieverding, C.H., Stanislas, M. and Snoek, J., "The Base Pressure Problem in Transonic Turbine Cascades," Journal of Engineering for Power, Vol 102, July 1980, pp. 711-718.

33. Stearns, E.M., "High Performance Turbine Aero Research Blade Leading Edge Thickness and Solidity Test," General Electric Technical Memorandum No. TM-69-586, 1971.

34. Stewart, W.L., Glassman, A.J., and Vanca, M.R., "Examination of Axial-flow Turbine Blade-Loading Characteristics Using Diffusion Parameters," ASME paper No. 67-WA/GT-8.

35. Turbine Design and Application. NASA SP-290, pp. 2-19.

36. Wysong, R.R., Prince, T.C., and Lenahan, D.T., "Turbine Design System," General Electric Aircraft Engines Internal Report, November 1978.

37. Xu, L. and Denton, J.D., "The Base Pressure and Loss of a Family of Four Turbine Blades, " ASME paper No. 87-GT-202.

38. Zaccaria, M.A., "Development of a Transonic Turbine Cascade Facility, " VPI Thesis , 1988.

39. Zeidan, O., " An Experimental Examination of the Effect of Trailing Edge Thickness on the Aerodynamic Performance of Gas Turbine Blades, " VPI Thesis, 1989.

40. Zweifel, O., "The Spacing of Turbo-Machine Blading, Especially with Large Angular Deflection," The Brown Boveri Review, December 1945, pp. 436-444. 


\subsection{Appendix}


Table 1. Comparison of blade design parameters

\begin{tabular}{|l|c|c|c|}
\hline Parameter & UN10 & Baseline & OV 10 \\
\hline Solidity change $(\Delta \sigma$, percent $)$ & -10 & 0 & +10 \\
\hline Solidity $(\sigma=\mathrm{c} / \mathrm{s})$ & 0.923 & 1.025 & 1.128 \\
\hline Loading $\left(\Psi_{z}\right)$ & 0.83 & 0.72 & 0.66 \\
\hline Throat $(\mathrm{t}, \mathrm{mm})$ & 14.34 & 13.12 & 12.03 \\
\hline Spacing $(\mathrm{s}, \mathrm{mm})$ & 40.67 & 37.16 & 34.06 \\
\hline Relative Rotation $($ deg. $\mathrm{CW})$ & 1.10 & 0.0 & -0.65 \\
\hline Stagger $($ deg.) & 39.24 & 38.50 & 37.43 \\
\hline Throat / Spacing $(\mathrm{t} / \mathrm{s})$ & 0.3525 & 0.3530 & 0.3530 \\
\hline Axial chord $(\mathrm{c}, \mathrm{mm})$ & 37.59 & 38.1 & 38.43 \\
\hline Inlet metal angle $\left(\beta_{m 1}\right.$, deg.) & 0.0 & 0.0 & 0.0 \\
\hline Exit metal angle $\left(\beta_{m 2}\right.$, deg. $)$ & -67.8 & -66.7 & -66.05 \\
\hline Unguided Turning $(\varepsilon$, deg.) & 7.5 & 9.6 & 16.2 \\
\hline Trailing edge wedge angle $(\delta$, deg.) & 8.5 & 8.5 & 8.5 \\
\hline Trailing edge thickness $(\mathrm{mm})$ & 1.09 & 1.09 & 1.09 \\
\hline Span $(\mathrm{h}, \mathrm{mm})$ & 152.40 & 152.40 & 152.40 \\
\hline Aspect ratio $(\mathrm{h} / \mathrm{c})$ & 4.05 & 4.00 & 3.97 \\
\hline Number of blades in cascade & 10 & 11 & 12 \\
\hline
\end{tabular}


Table 2. Computational input parameters and results

\begin{tabular}{|l|c|c|c|}
\hline Parameter & UN10 & Baseline & OV10 \\
\hline Inlet Mach number $\left(M_{1}\right)$ & .21 & .21 & .21 \\
\hline Exit Mach number $\left(M_{\text {exi }}\right)$ & 1.22 & 1.22 & 1.23 \\
\hline Inlet air angle $\left(\beta_{1}\right.$, deg., calculated $)$ & 0.0 & 0.0 & 0.0 \\
\hline Exit air angle $\left(\beta_{2}\right.$, deg., calculated $)$ & -65.2 & -66.2 & -65.9 \\
\hline Number of calculation nodes & 3404 & 1848 & 3207 \\
\hline Number of triangles or quadrilaterals & 6470 & 3388 & 6063 \\
\hline Maximum error, $E M A X$ & 0.009 & 0.007 & 0.007 \\
\hline Average error, $E A V E$ & 0.001 & 0.001 & 0.001 \\
\hline Ratio of specific heats, $\gamma$ & 1.40 & 1.40 & 1.40 \\
\hline Gas constant $(R, \mathrm{~N} \mathrm{~m} / \mathrm{kg} \mathrm{K})$ & 287 & 287 & 287 \\
\hline Upstream Total Temperature $\left(T_{t \mathrm{t}}, \mathrm{K}\right)$ & 255 & 255 & 255 \\
\hline Upstream Total Pressure $\left(P_{\mathrm{t} 1}, \mathrm{kpa}\right)$ & 469 & 469 & 469 \\
\hline
\end{tabular}


Table 3. Uncertainty analysis summary

Baseline Cascade, 95 percent confidence

\begin{tabular}{|l|c|c|c|c|}
\hline \multicolumn{1}{|l}{ Parameter } & $M_{2 i s}=\mathbf{0 . 8}$ & \multicolumn{1}{c|}{$M_{2 i s}=1.0$} & $M_{2 i s}=1.2$ & $M_{2 i s}=1.35$ \\
\hline$\left(\partial L_{c} / \partial P_{o t m}\right),(1 / \mathrm{psi})$ & -0.036 & -0.28 & -0.382 & -0.664 \\
\hline$\delta P_{a t m}, \mathrm{psi}$ & 0.15 & 0.15 & 0.15 & 0.15 \\
\hline$\left(\partial L_{c} / \partial M\right)$ & 0.125 & 0.143 & -7.174 & -17.714 \\
\hline$\delta M$ & 0.03 & 0.03 & 0.035 & 0.04 \\
\hline$\left(\partial L_{c} / \partial P_{t 1}\right),(1 /$ Volt $)$ & -0.5 & -0.063 & -0.423 & -4.423 \\
\hline$\delta P_{t 1}$, Volts & 0.01 & 0.01 & 0.01 & 0.01 \\
\hline$\left(\partial L_{c} / \partial \Delta P_{t}\right),(1 /$ Volt $)$ & 0.167 & 0.30 & 0.667 & 1.467 \\
\hline$\delta \Delta P_{t}$, Volts & 0.01 & 0.01 & 0.01 & 0.01 \\
\hline$\left(\partial L_{c} / \partial P_{2}\right),(1 / \mathrm{psi})$ & -0.006 & -0.005 & 0.905 & 1.403 \\
\hline$\delta P_{2}, \mathrm{psi}$ & 0.15 & 0.15 & 0.15 & 0.15 \\
\hline RESULT & & & & \\
\hline$\left.\delta L_{c}\right|_{S T N 1}$ & 0.008 & 0.04 & 0.257 & 0.773 \\
\hline$\left.\delta L_{c}\right|_{S T N 2}$ & 0.007 & 0.039 & 0.146 & 0.235 \\
\hline$\left.\delta L_{c}\right|_{S T N 1}$, pct.(Baseline) & 0.8 & 2.6 & 8.6 & 17.0 \\
\hline$\left.\delta L_{c}\right|_{S T N 2}$, pct.(Baseline) & 0.7 & 2.6 & 4.9 & 5.2 \\
\hline
\end{tabular}




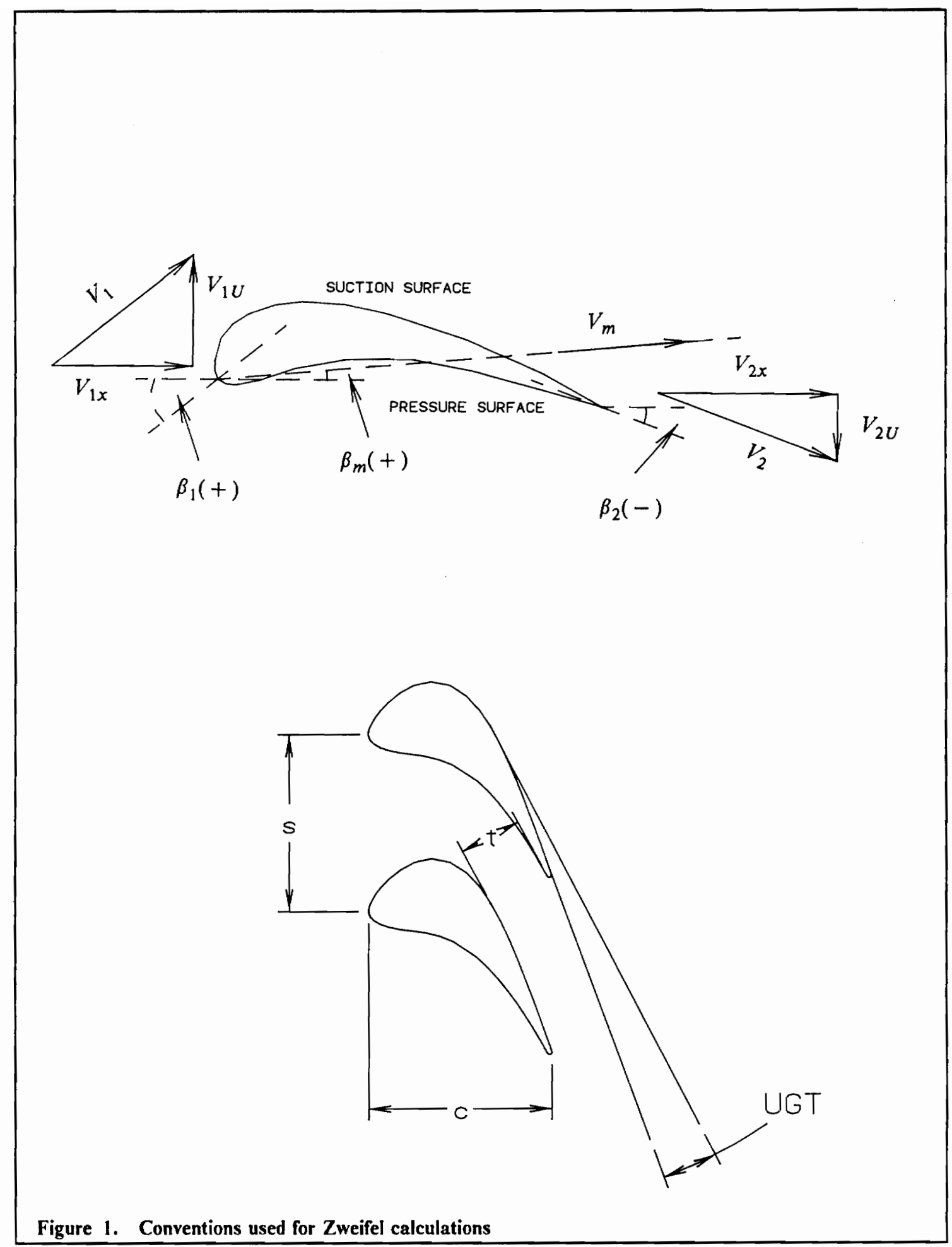




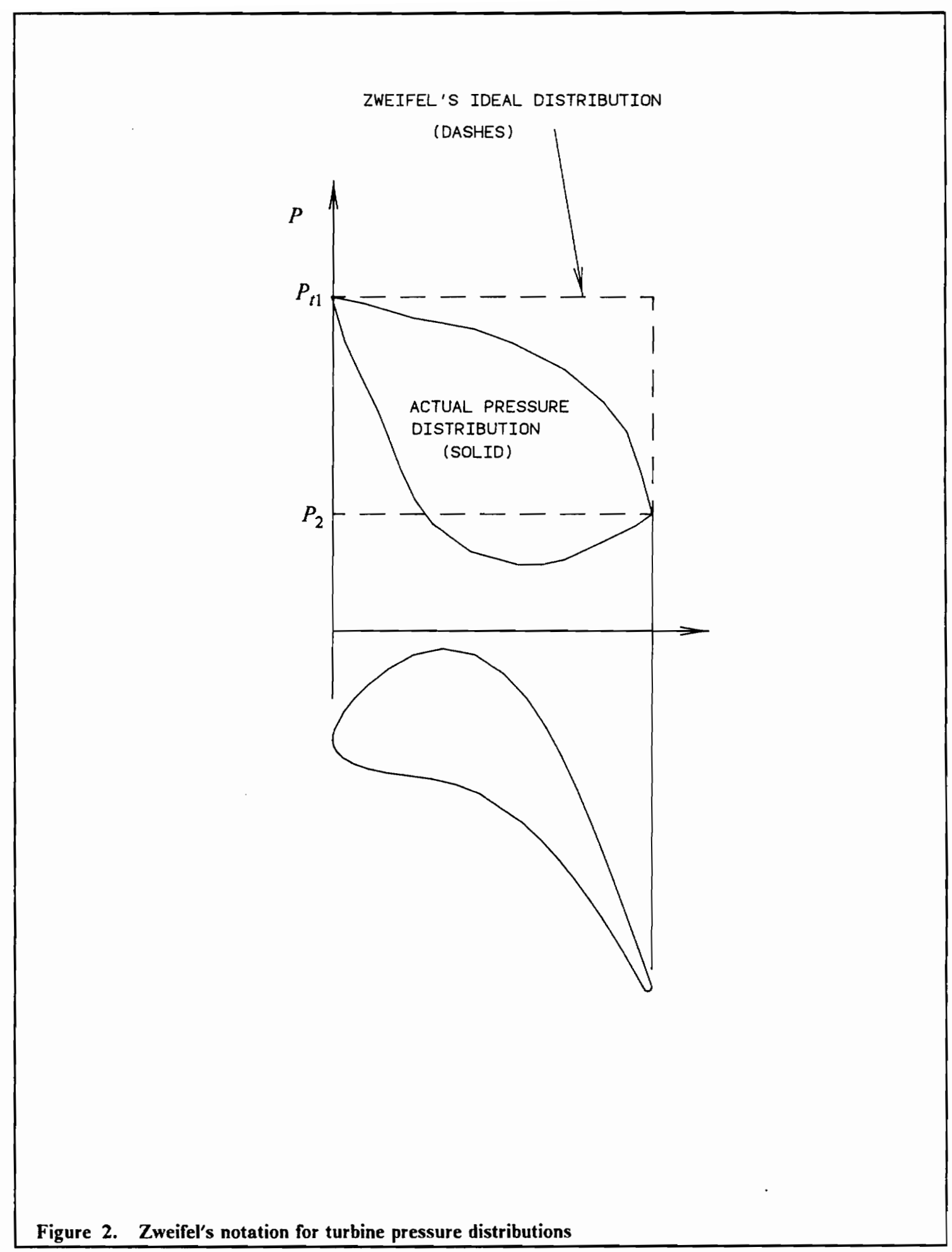



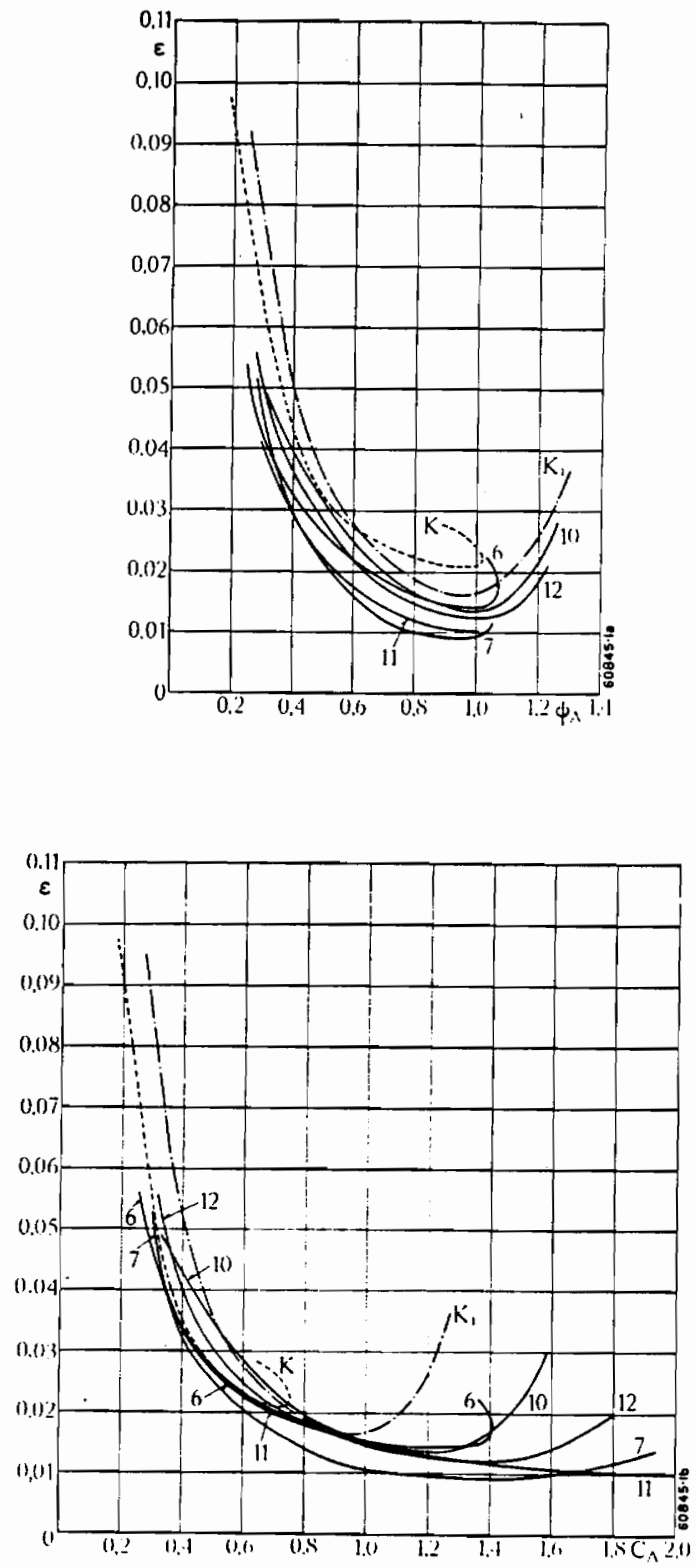

Figure 3. Drag-lift ratios $(\varepsilon)$ of airfoil cascades:

Top: $\varepsilon$ vs. Zweifel coefficient.

Bottom: $\varepsilon$ vs. Lift coefficient.

From Reference 40. 

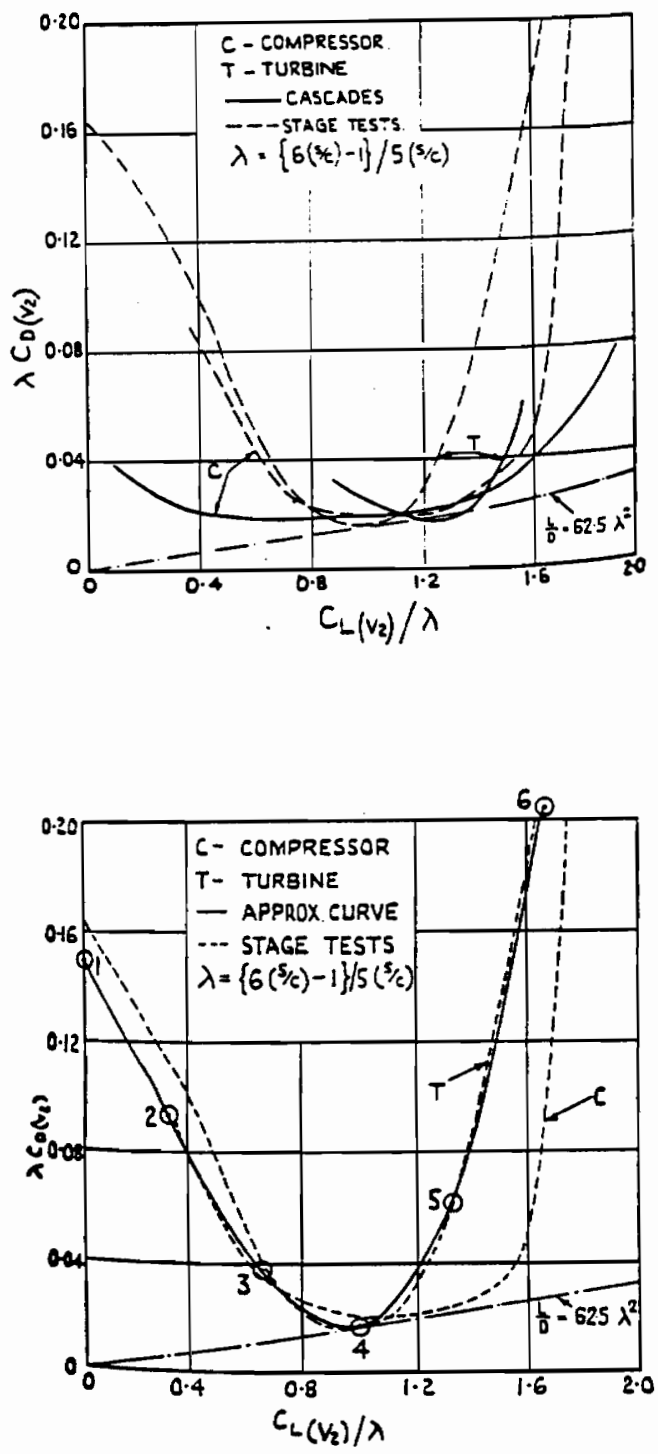

Figure 4. Relationship between Howell's Lift and Drag coefficients:

Top: Modified Lift and Drag coefficients for various blade configurations. Bottom: Howell's curve fit. From Reference 19. 

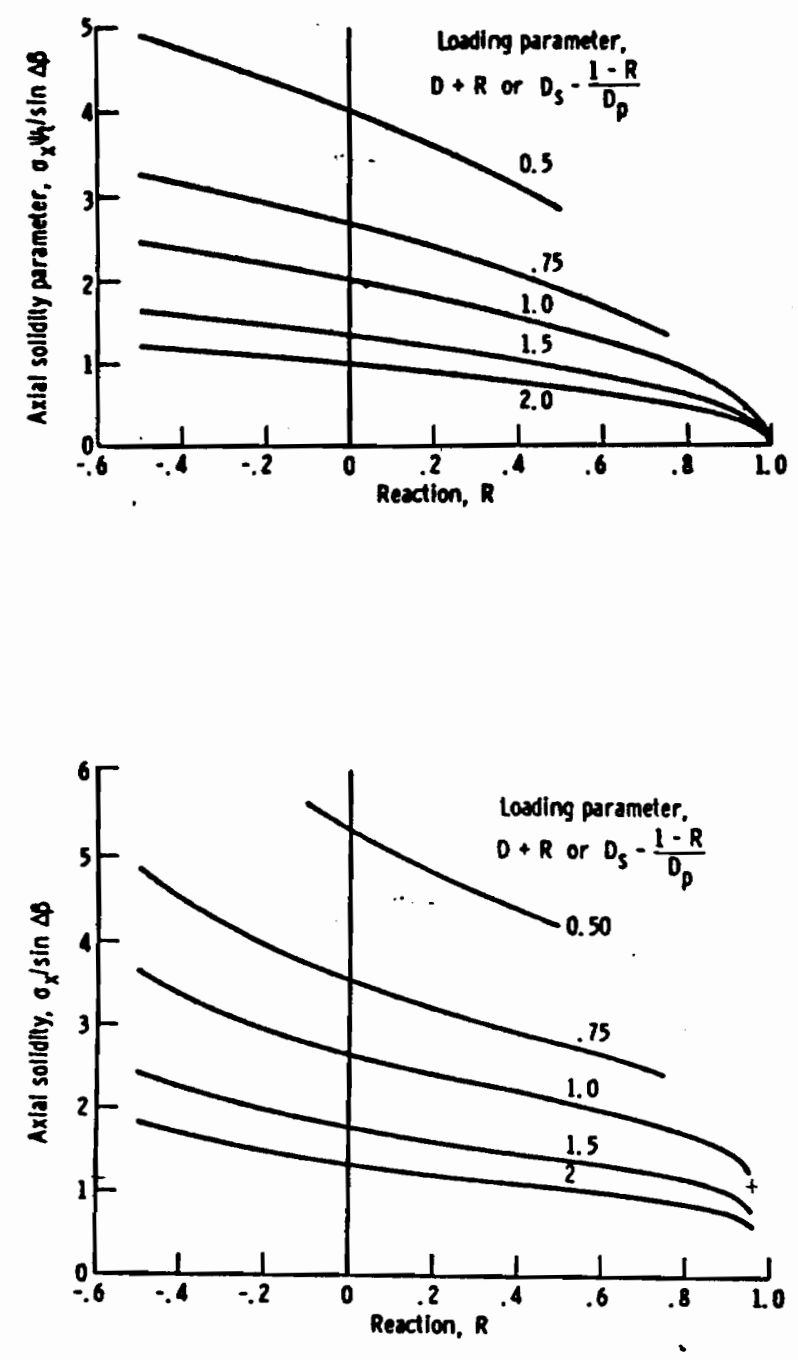

Figure 5. Axial solidity parameter vs. reaction and diffusion:

Top: Axial solidity parameter as a function of reaction and diffusion.

Bottom: Variation of axial solidity using the assumed loading model. From Reference 34. 


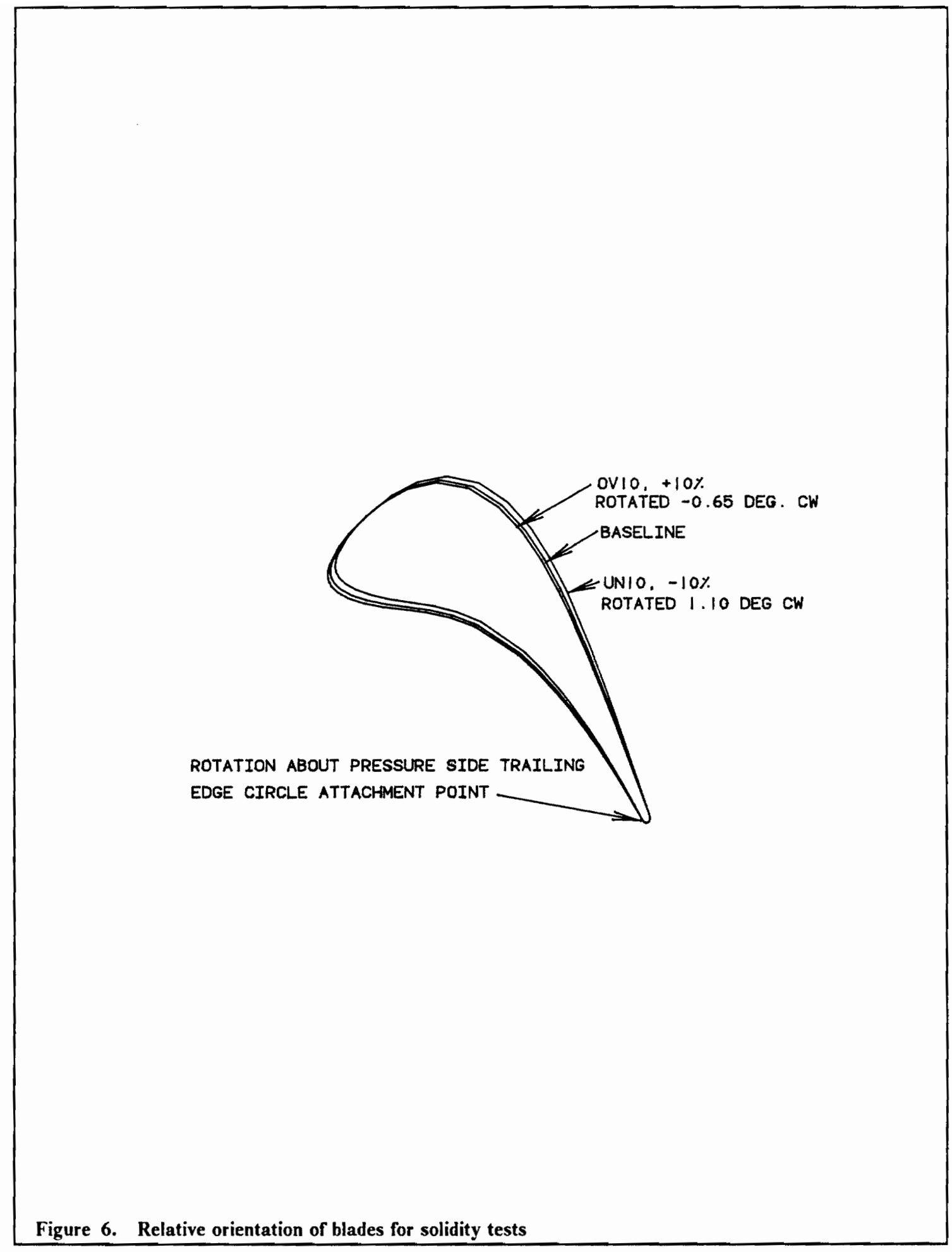




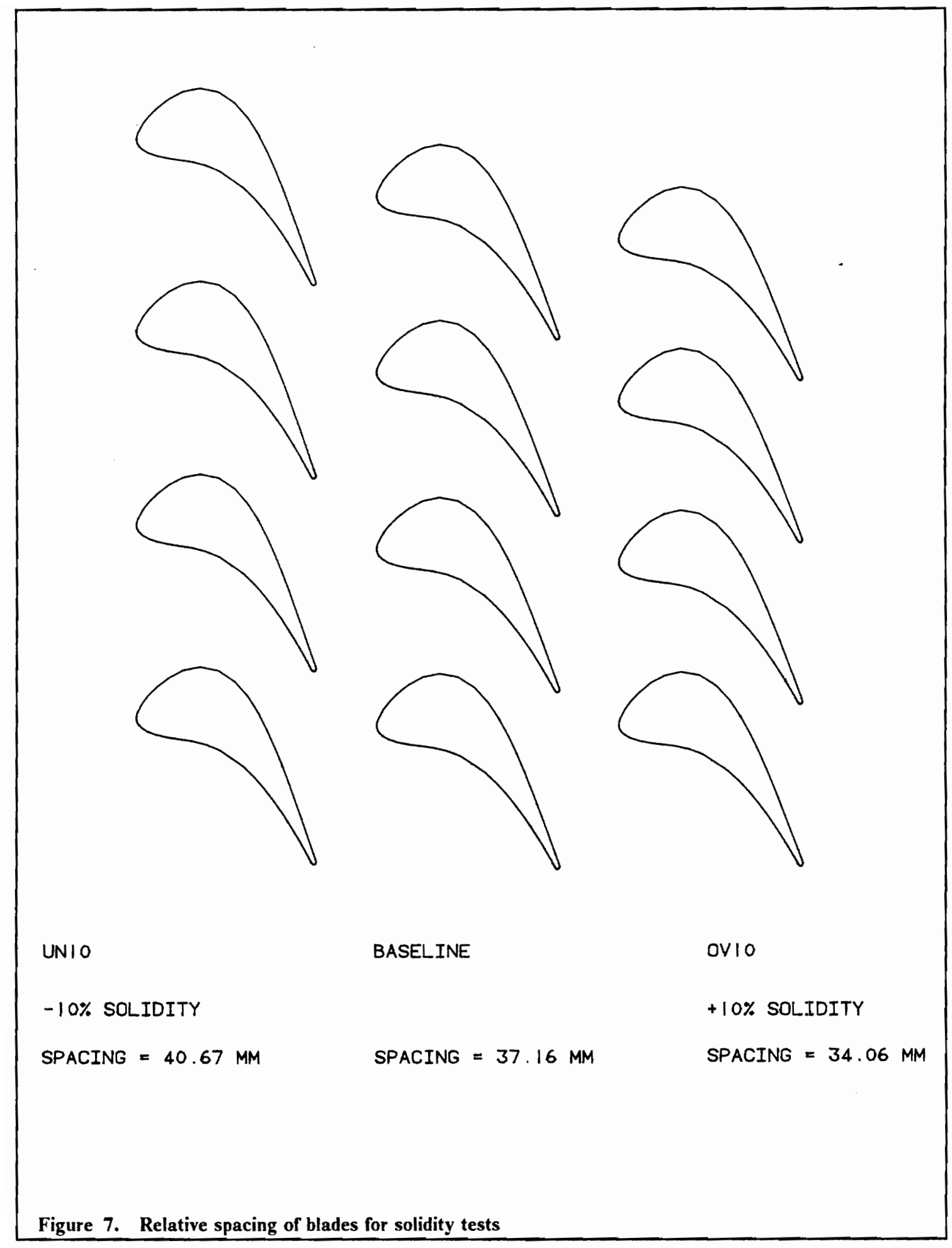




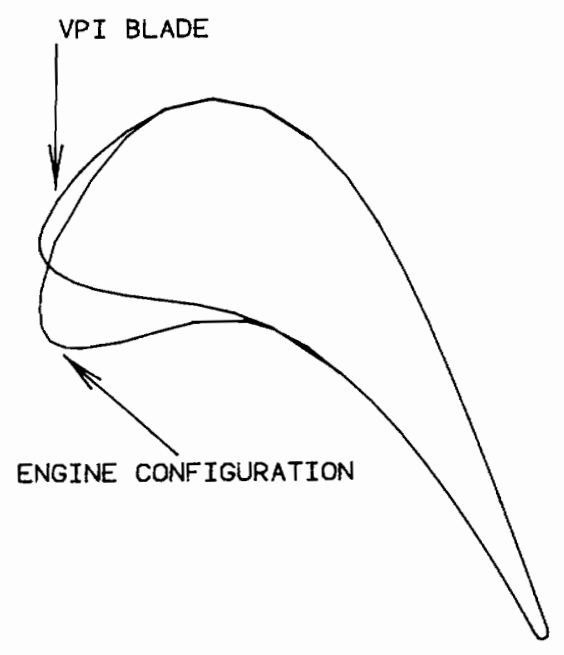

Figure 8. Physical comparison of original blade and VPI blade: VPI blade: $\beta_{m 1}=0^{\circ}, \beta_{m 2}=-68^{\circ}$.

Engine configuration: $\beta_{m 1}=55^{\circ}, \beta_{m 2}=-68^{\circ}$. 


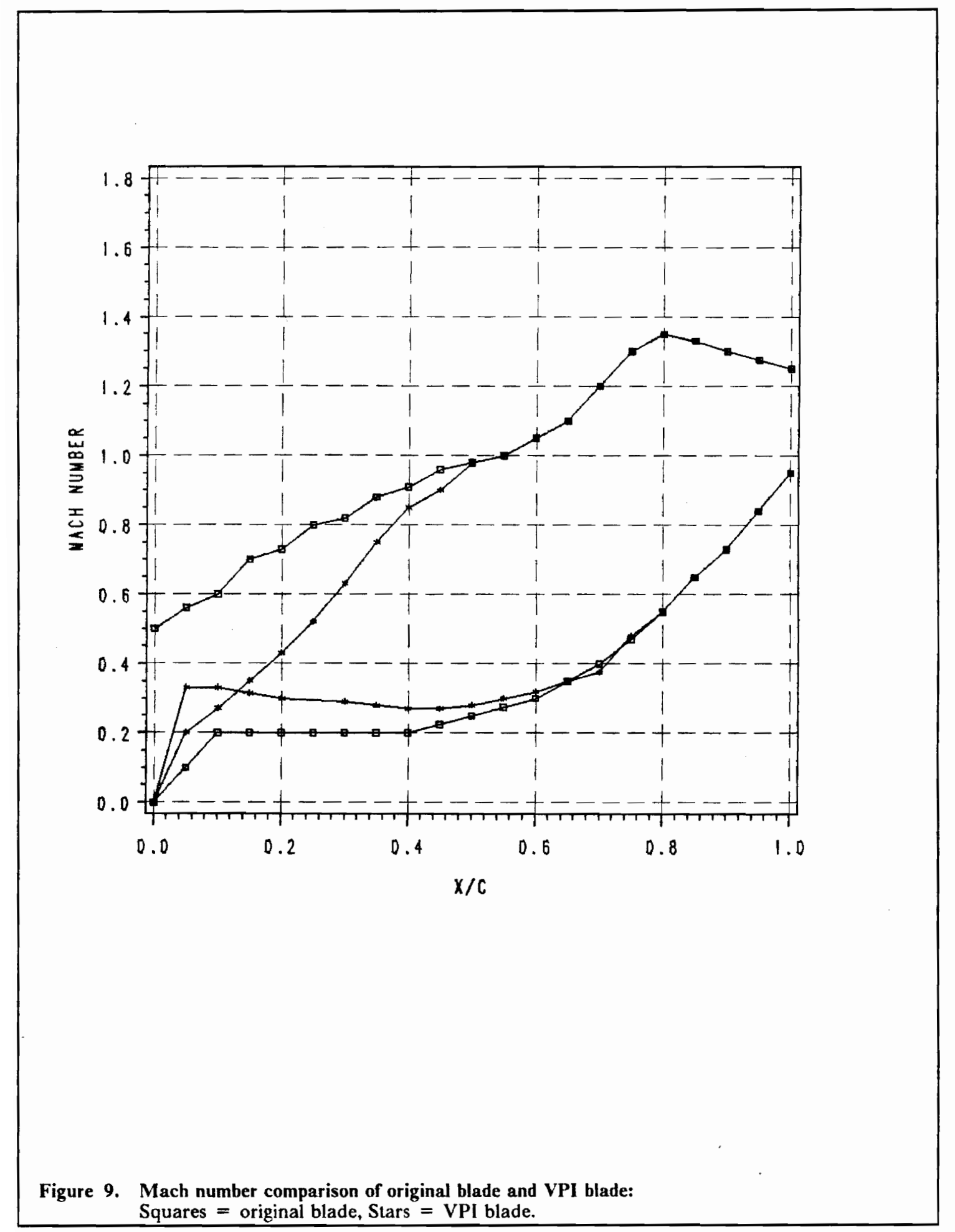




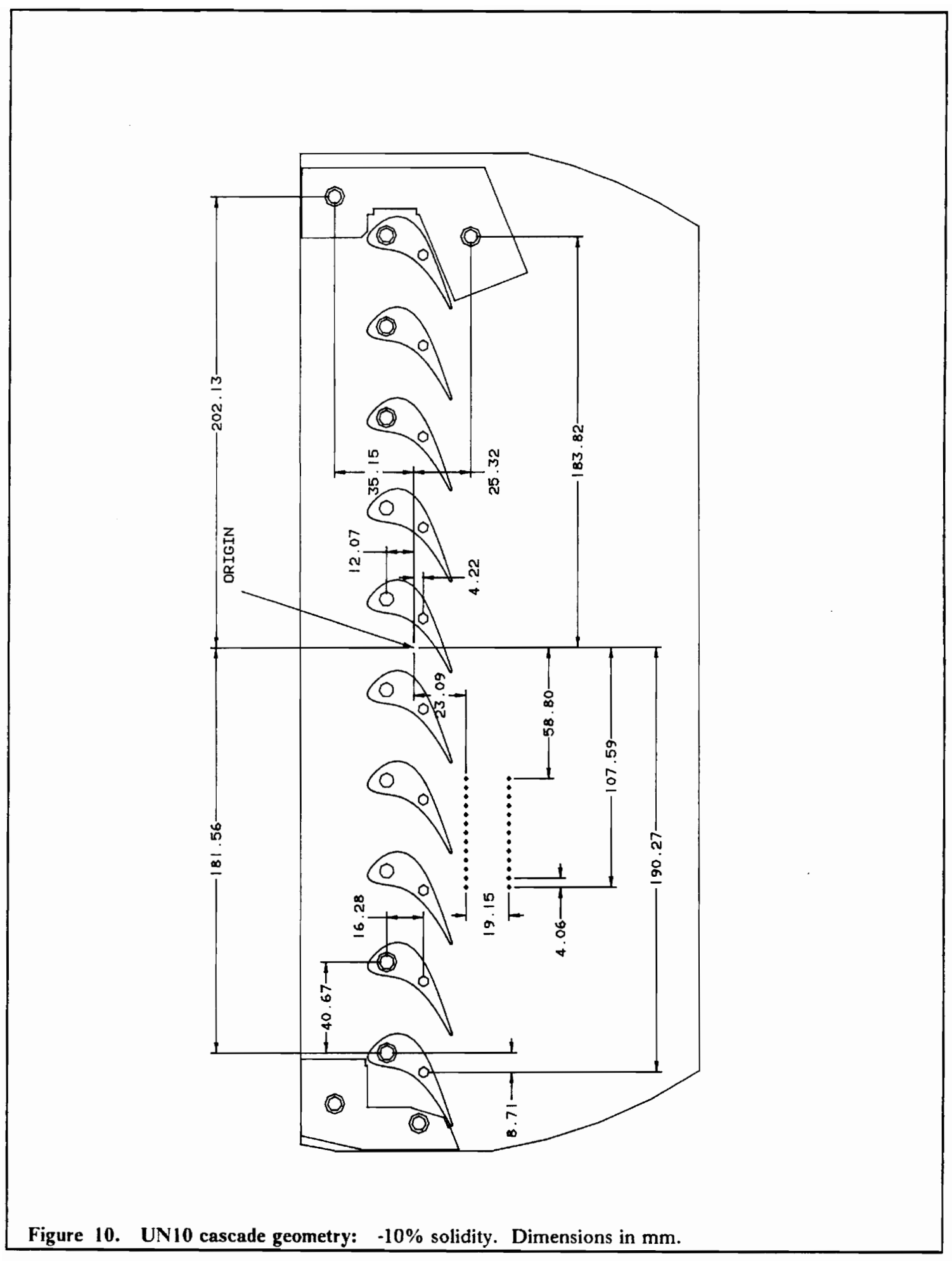

Appendix

79 


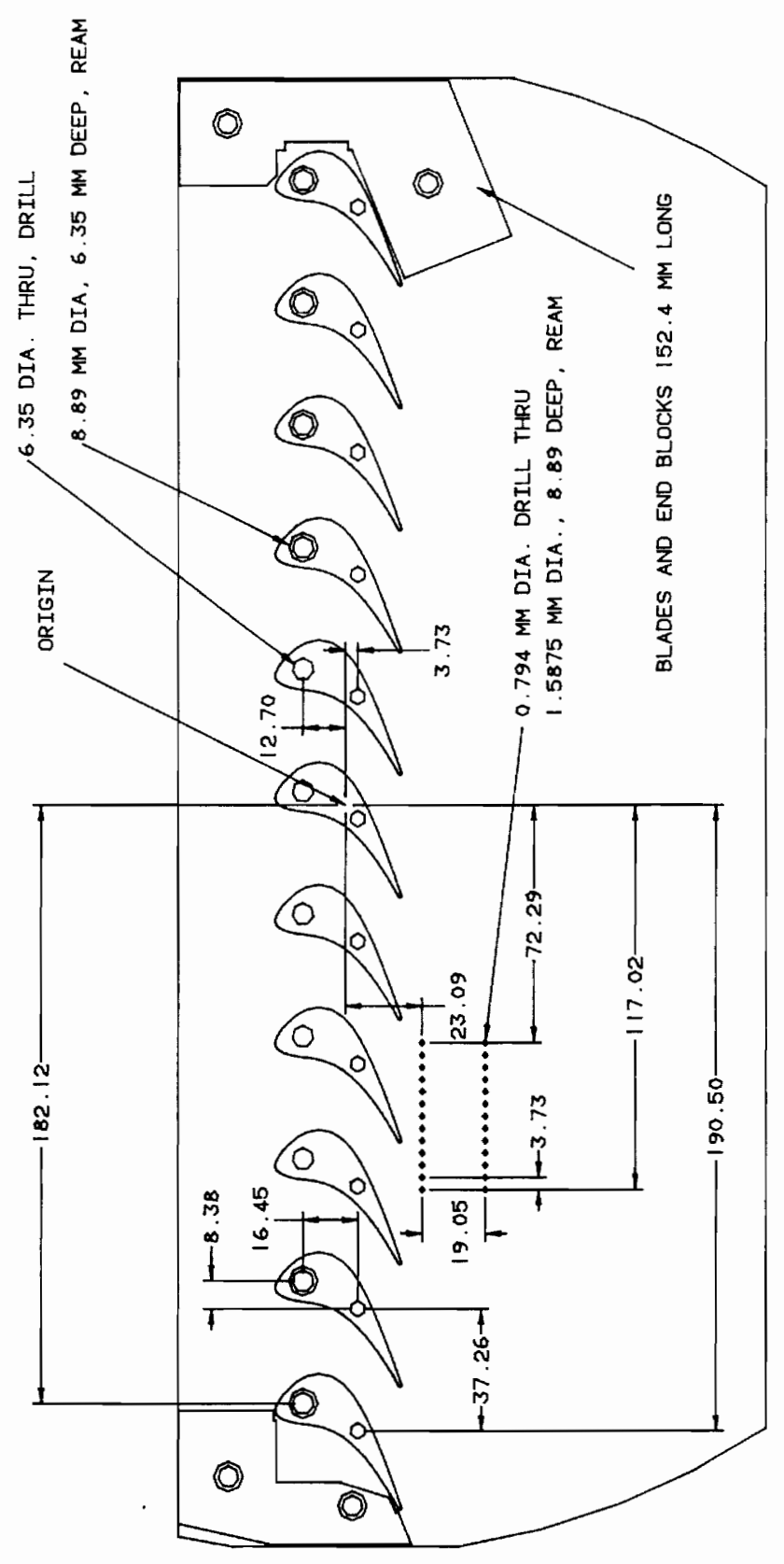

Figure 11. Baseline cascade geometry: Baseline solidity. Dimensions in $\mathrm{mm}$. 


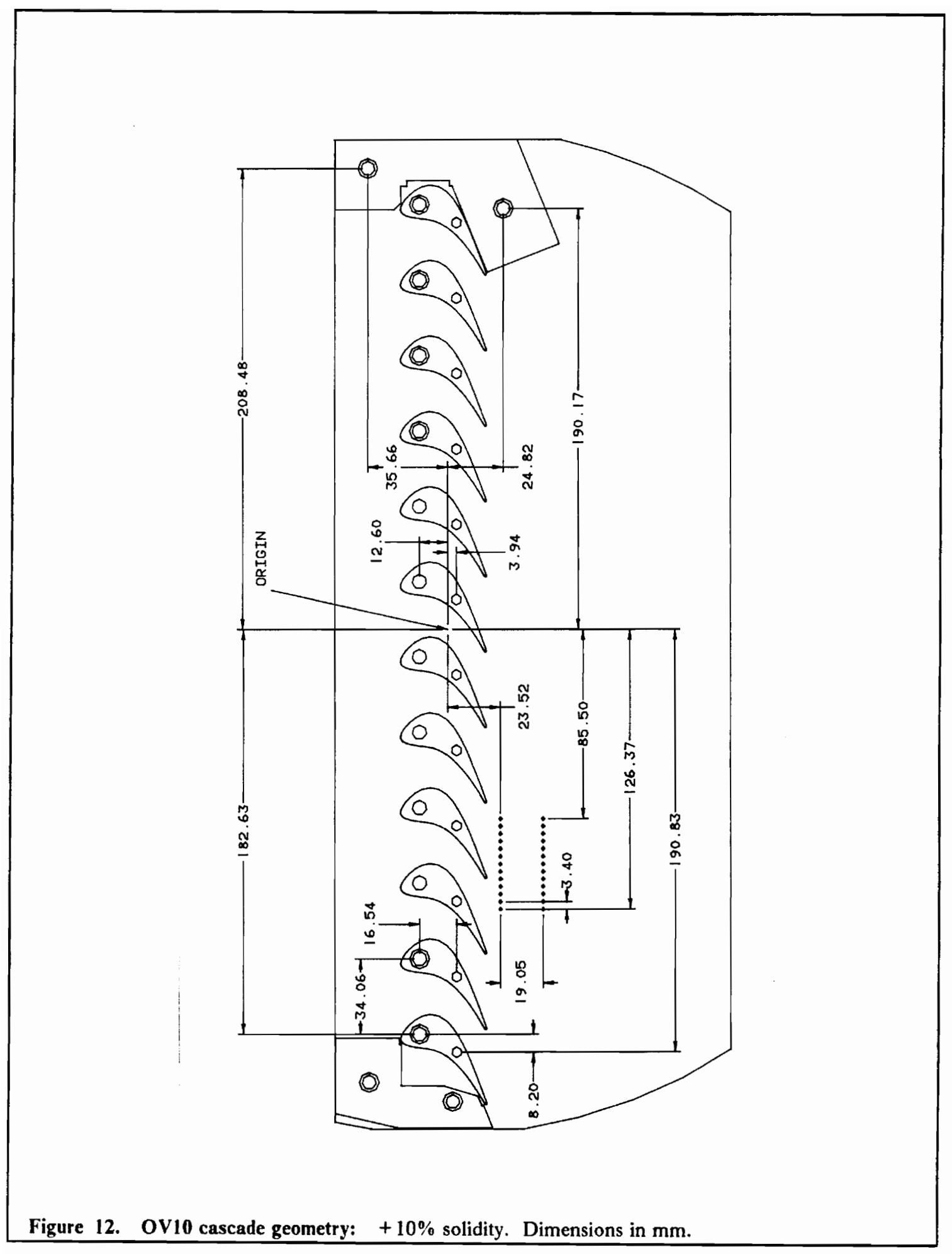

Appendix

81 


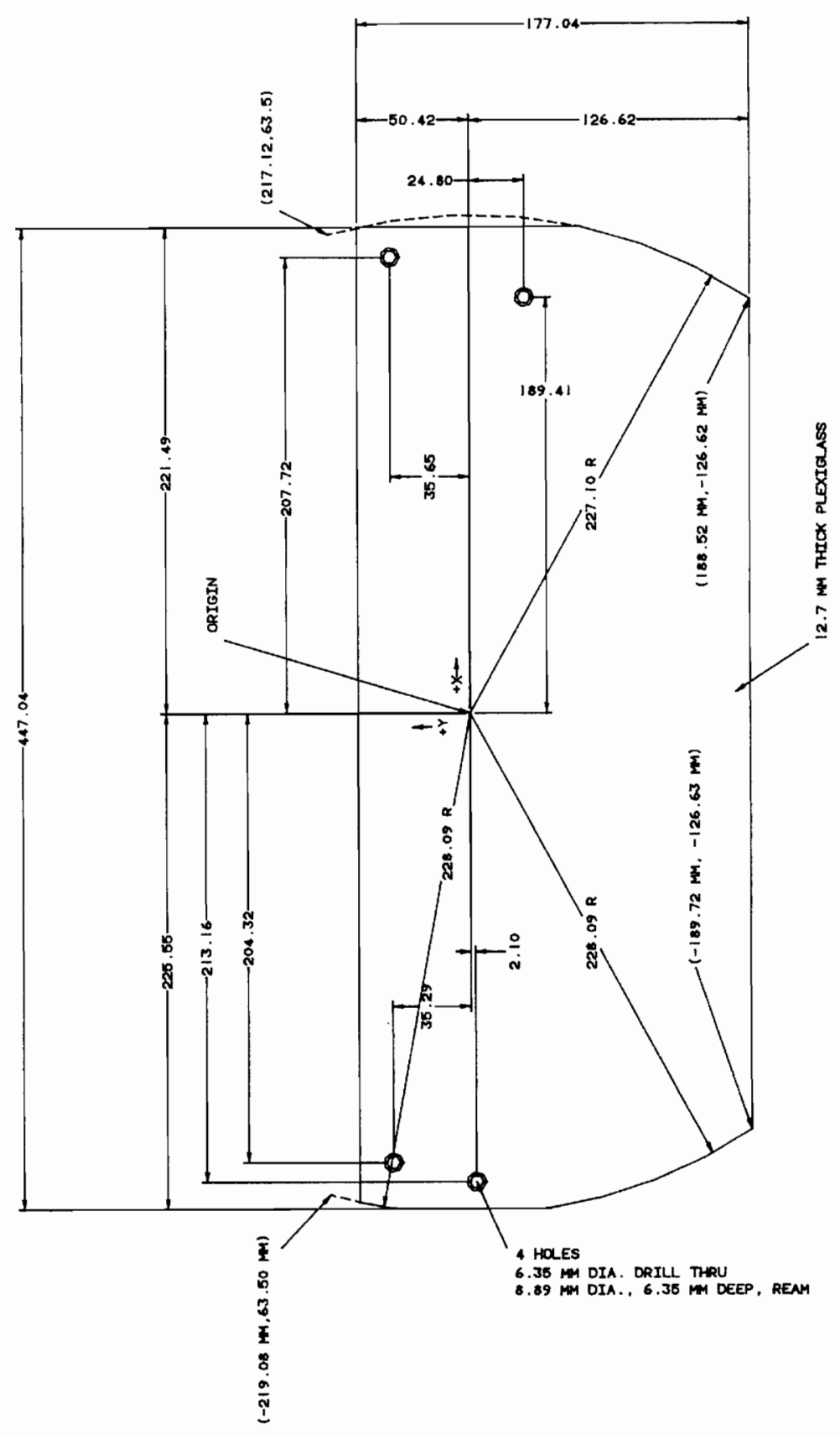

Figure 13. Endplate outer dimensions: Dimensions in $\mathrm{mm}$. 


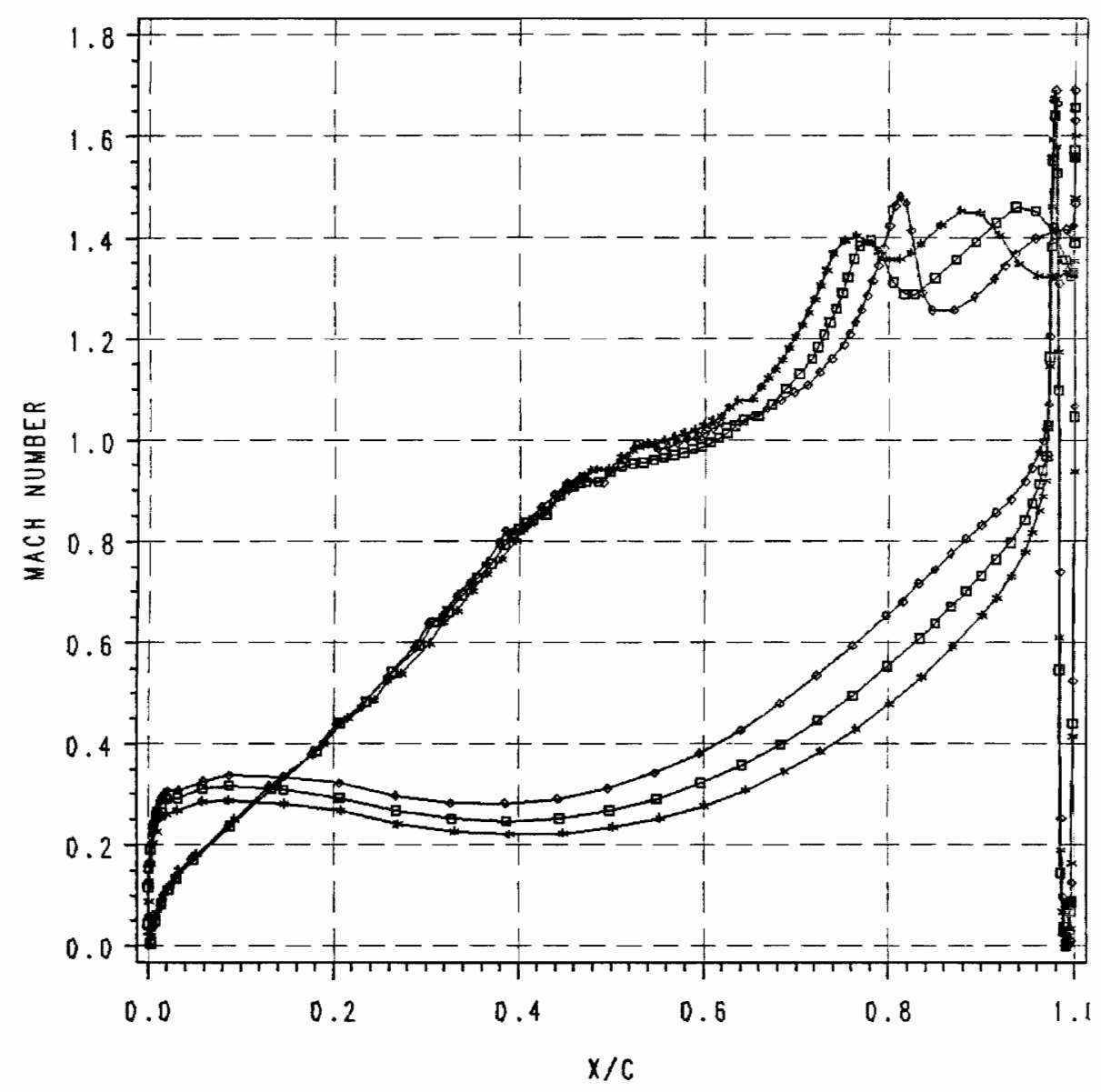

Figure 14. Predicted blade surface Mach number distributions: Stars: UN10, $\sigma=0.923, M_{2 i s}=1.30$

Squares: Baseline, $\sigma=1.025, M_{2 i s}=1.30$

Diamonds: OV10, $\sigma=1.128, M_{2 i s}=1.29$

Boundary conditions: $P_{t 1}=469 \mathrm{kpa}, P_{b a c k}=177 \mathrm{kpa}$. 


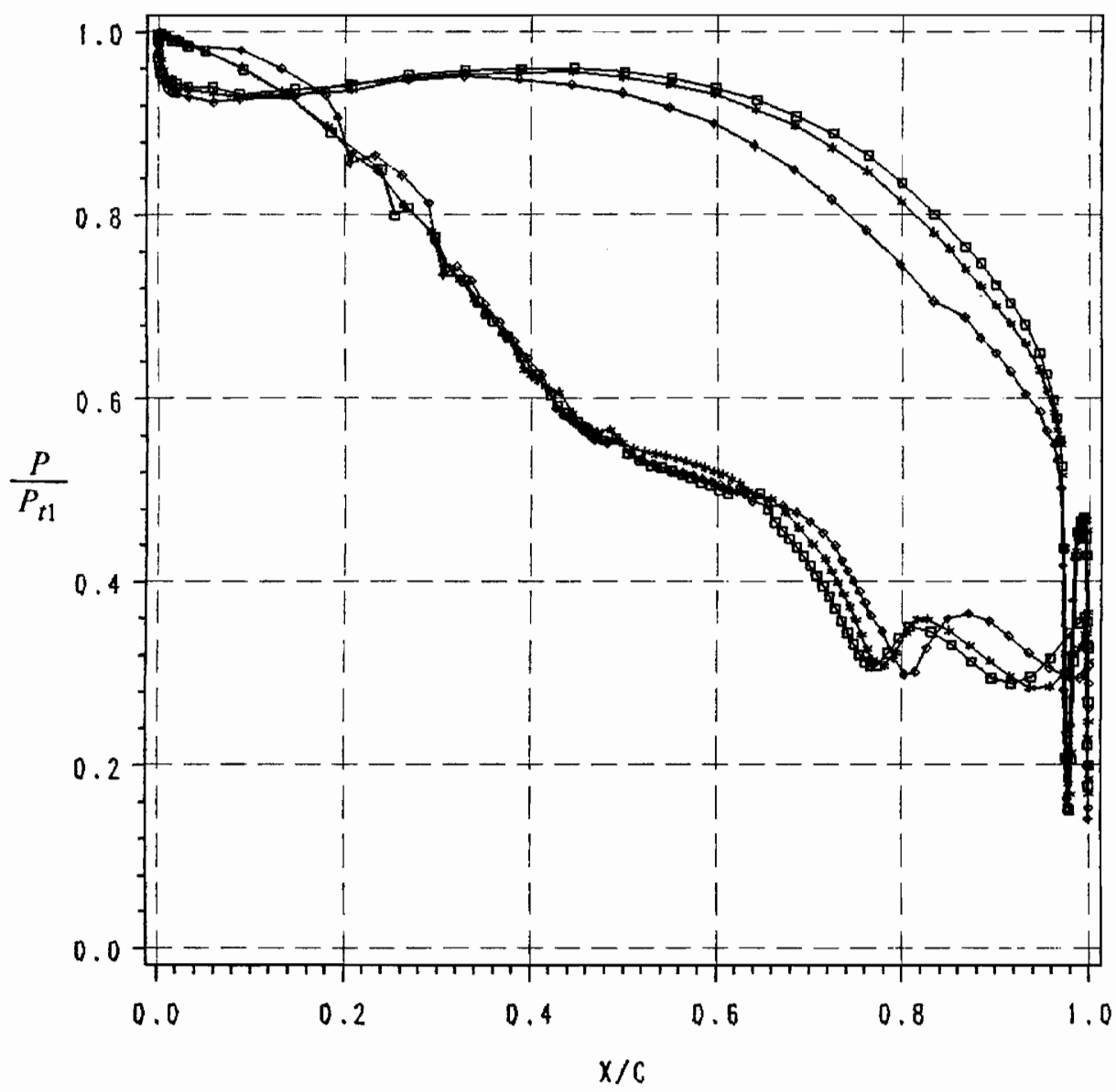

Figure 15. Predicted blade surface static pressure distributions:

Stars: UN10, $\sigma=0.923, P_{t 1} / P_{2}=2.77$

Squares: Baseline, $\sigma=1.025, P_{t 1} / P_{2}=2.77$

Diamonds: OV10, $\sigma=1.128, P_{t 1} / P_{2}=2.73$

Boundary conditions: $P_{\mathrm{t} 1}=469 \mathrm{kpa}, P_{\text {back }}=177 \mathrm{kpa}$. 


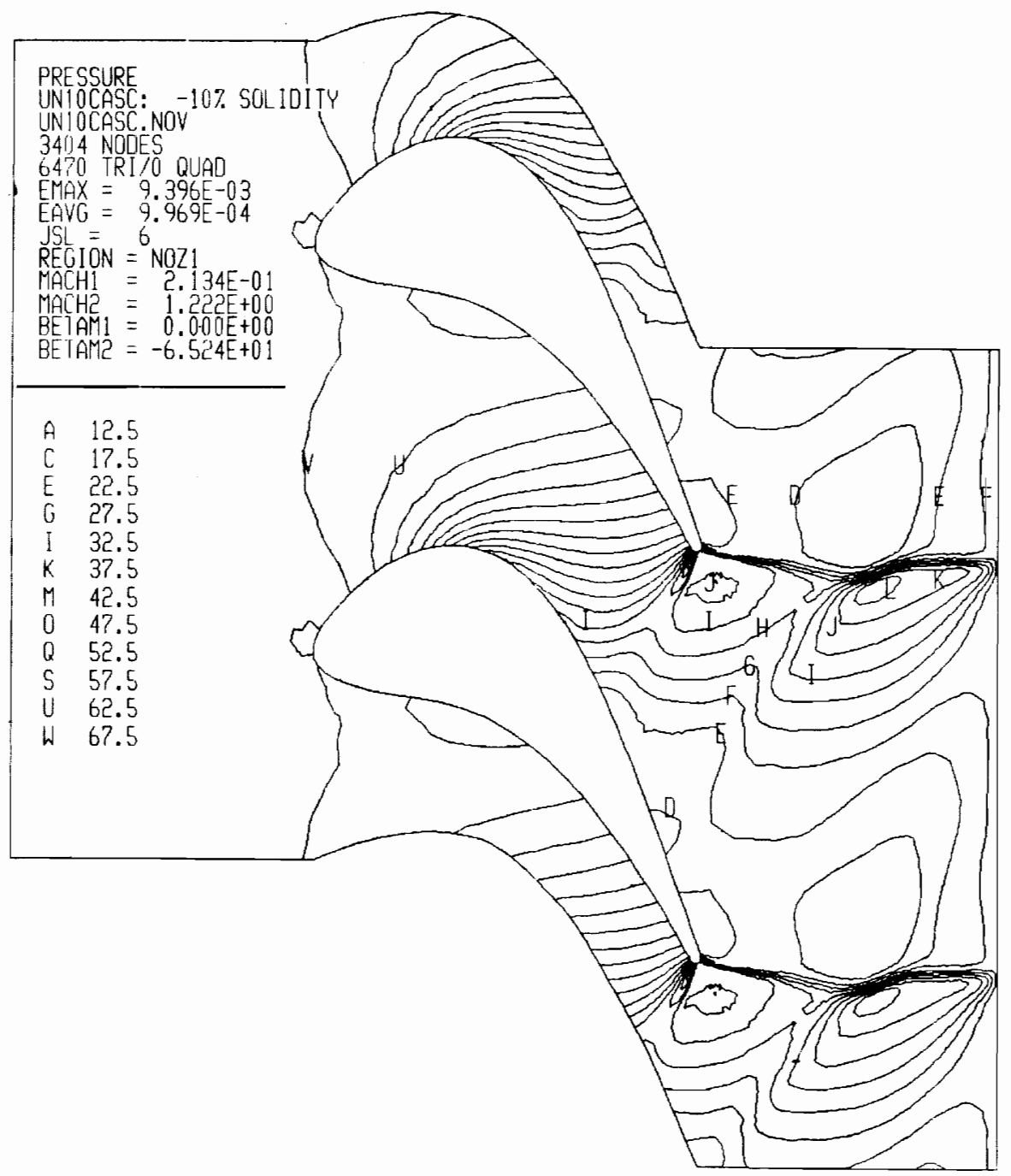

Figure 16. Predicted pressure contours, UN10 cascade, $\mathbf{P}_{t 1} / \mathbf{P}_{\mathbf{2}}=\mathbf{2 . 7 7}$ :

Boundary conditions: $P_{t} / P_{b a c k}=2.65$

$M_{\text {exit }}=1.22,\left(M_{2 i s}=1.29\right)$ 


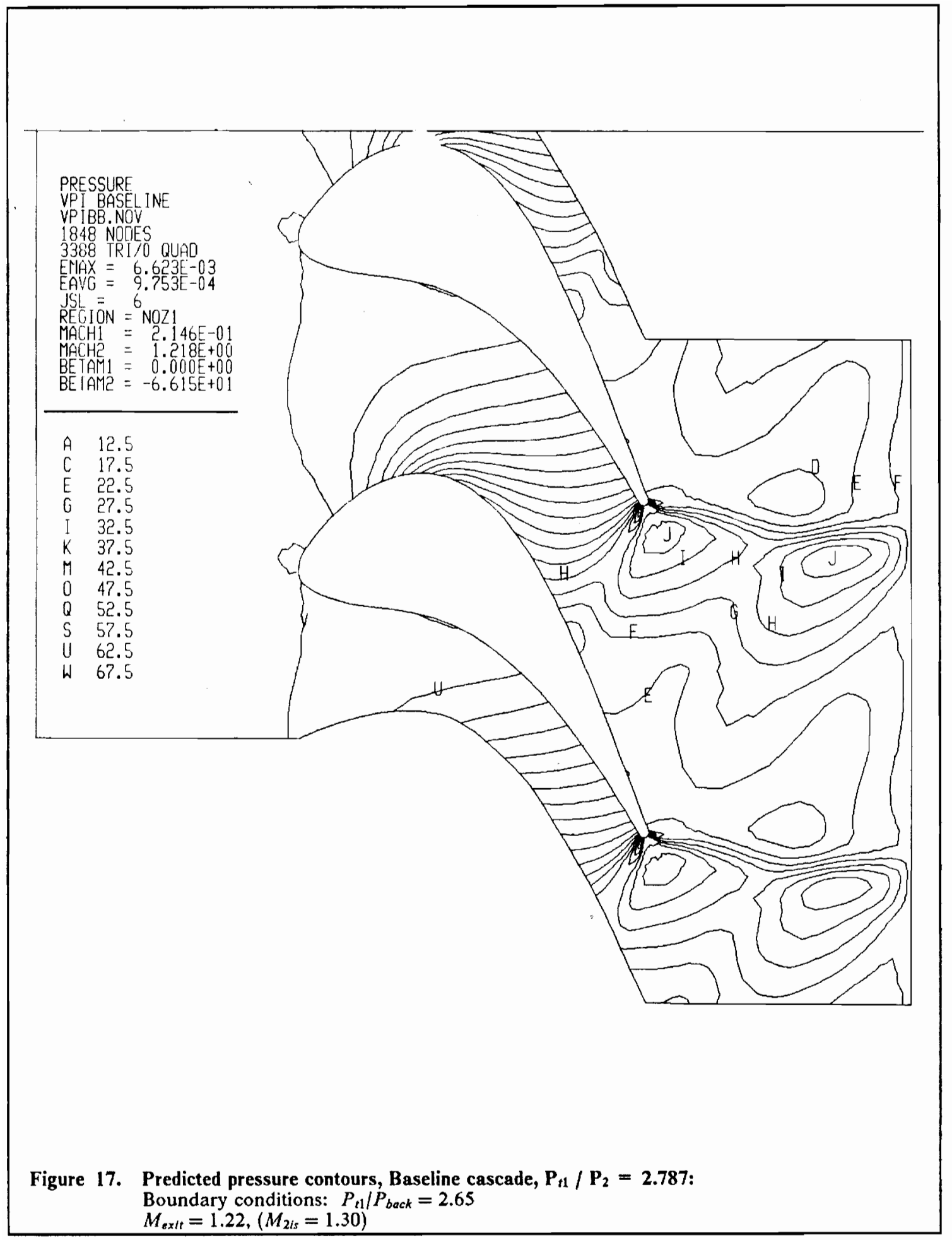




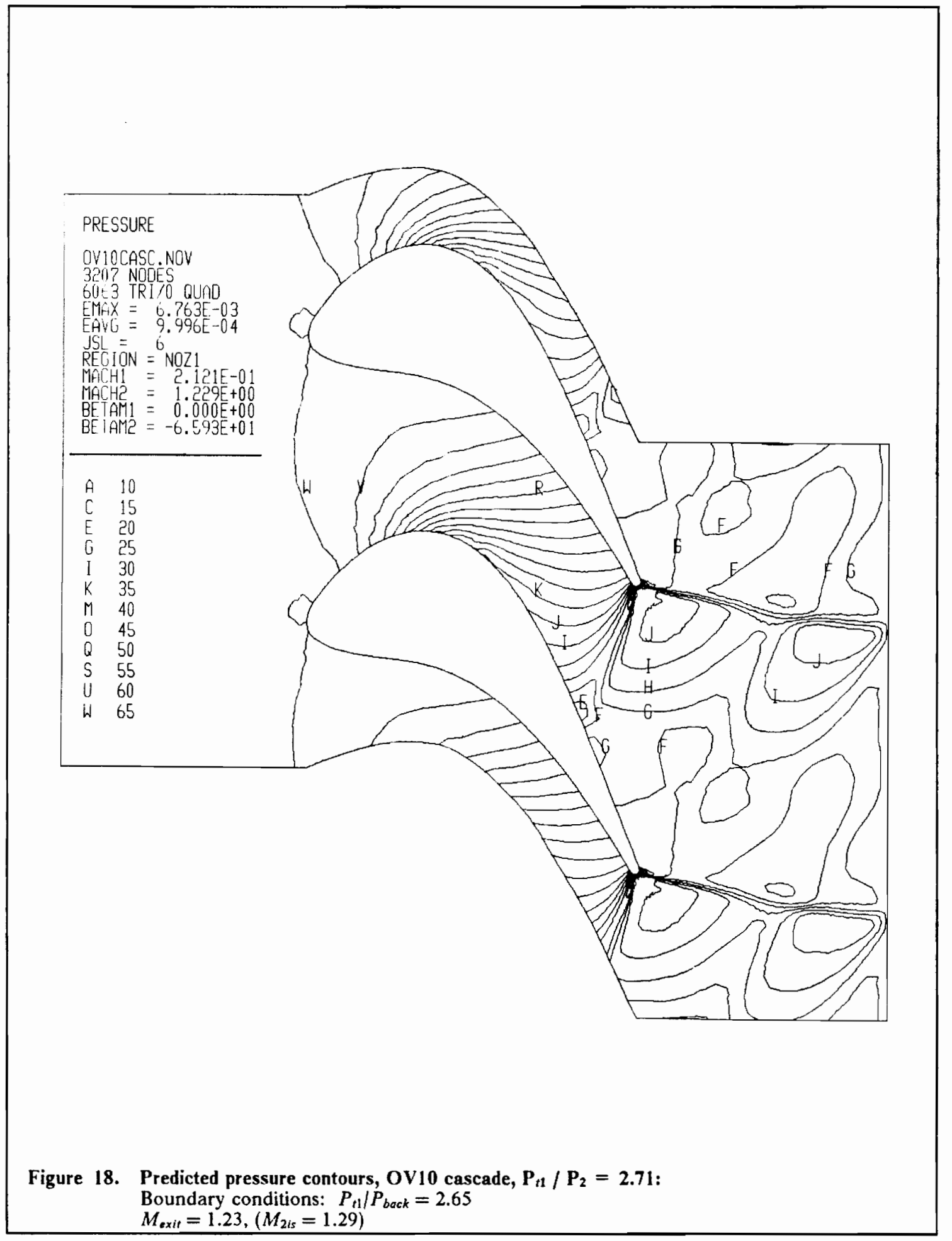




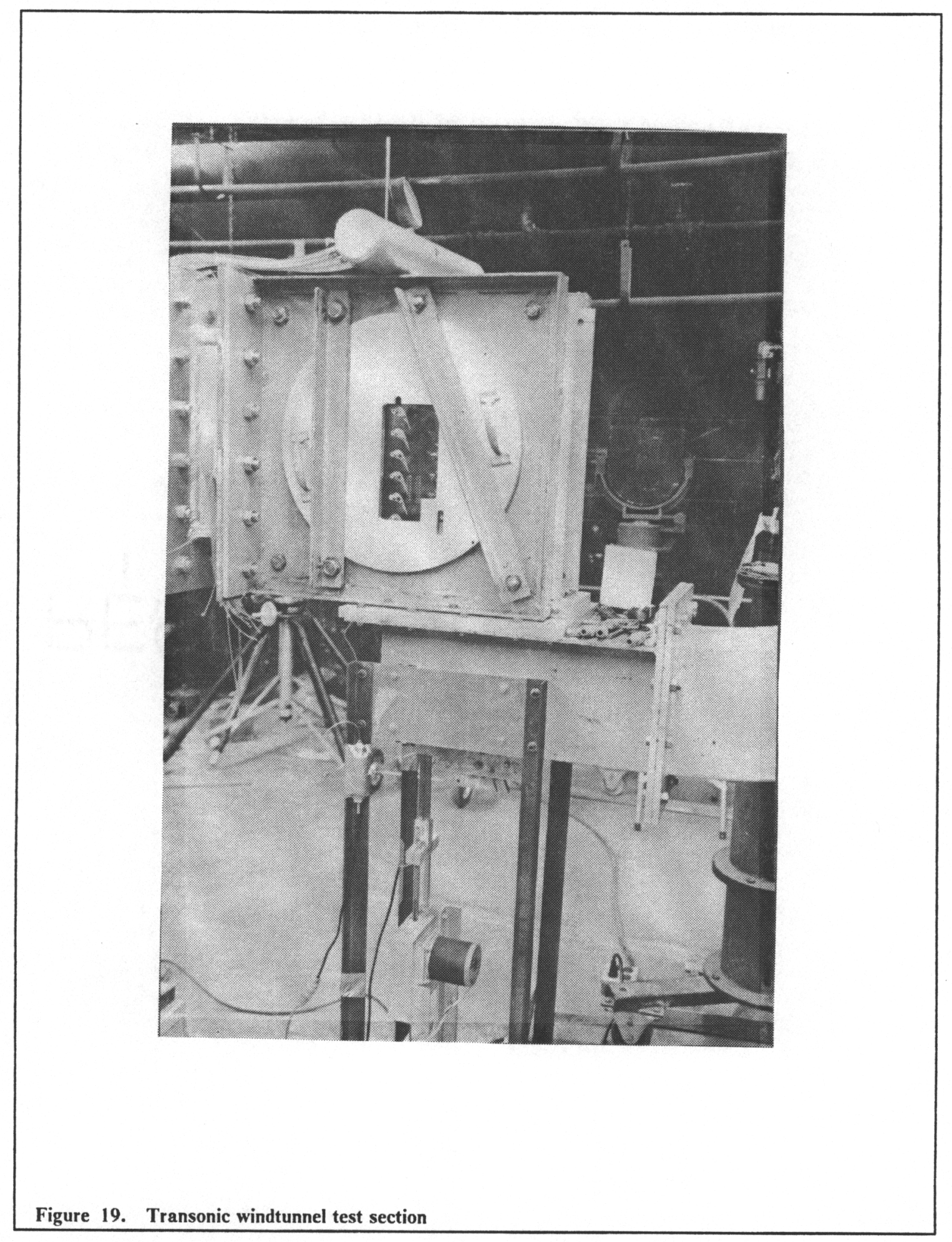




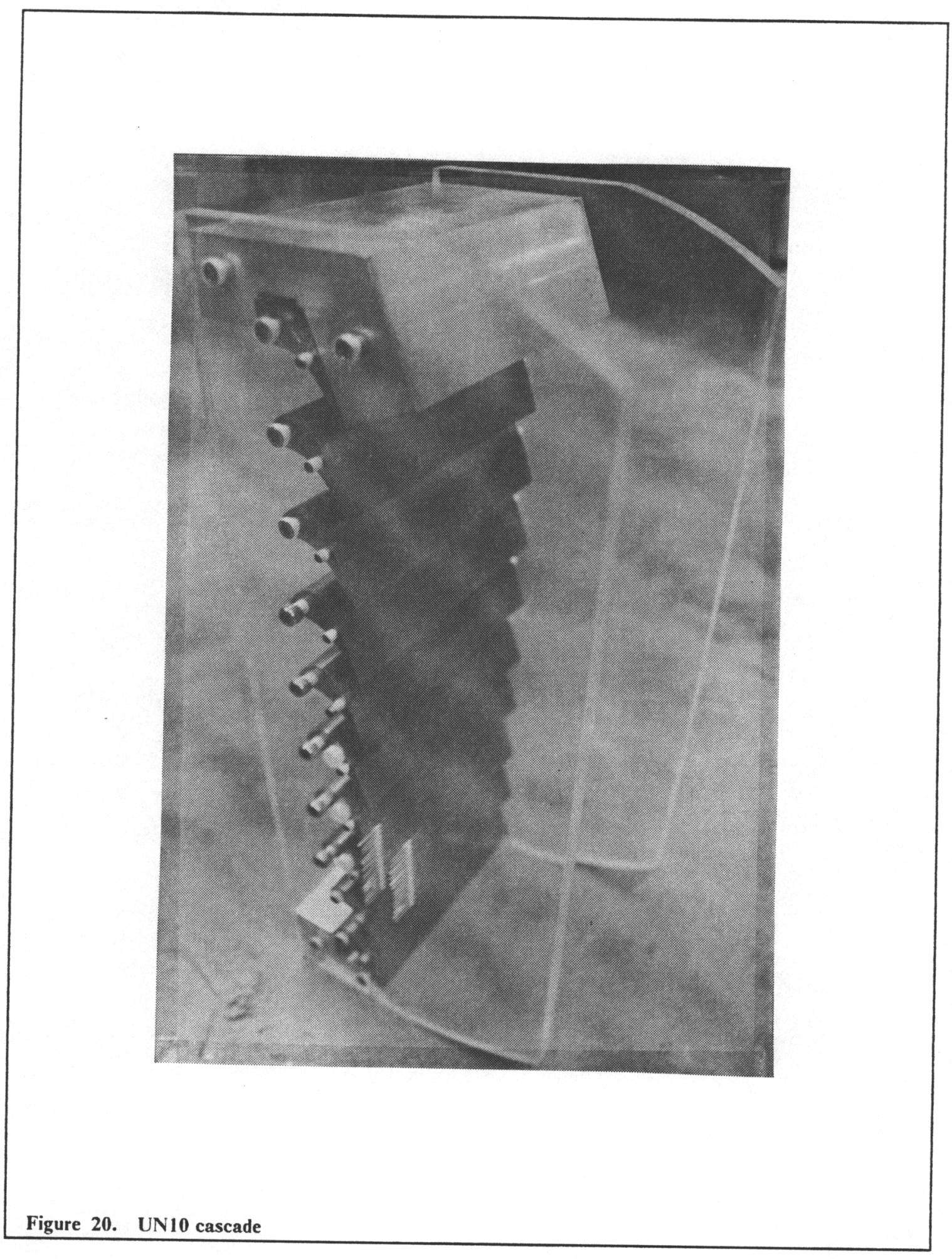




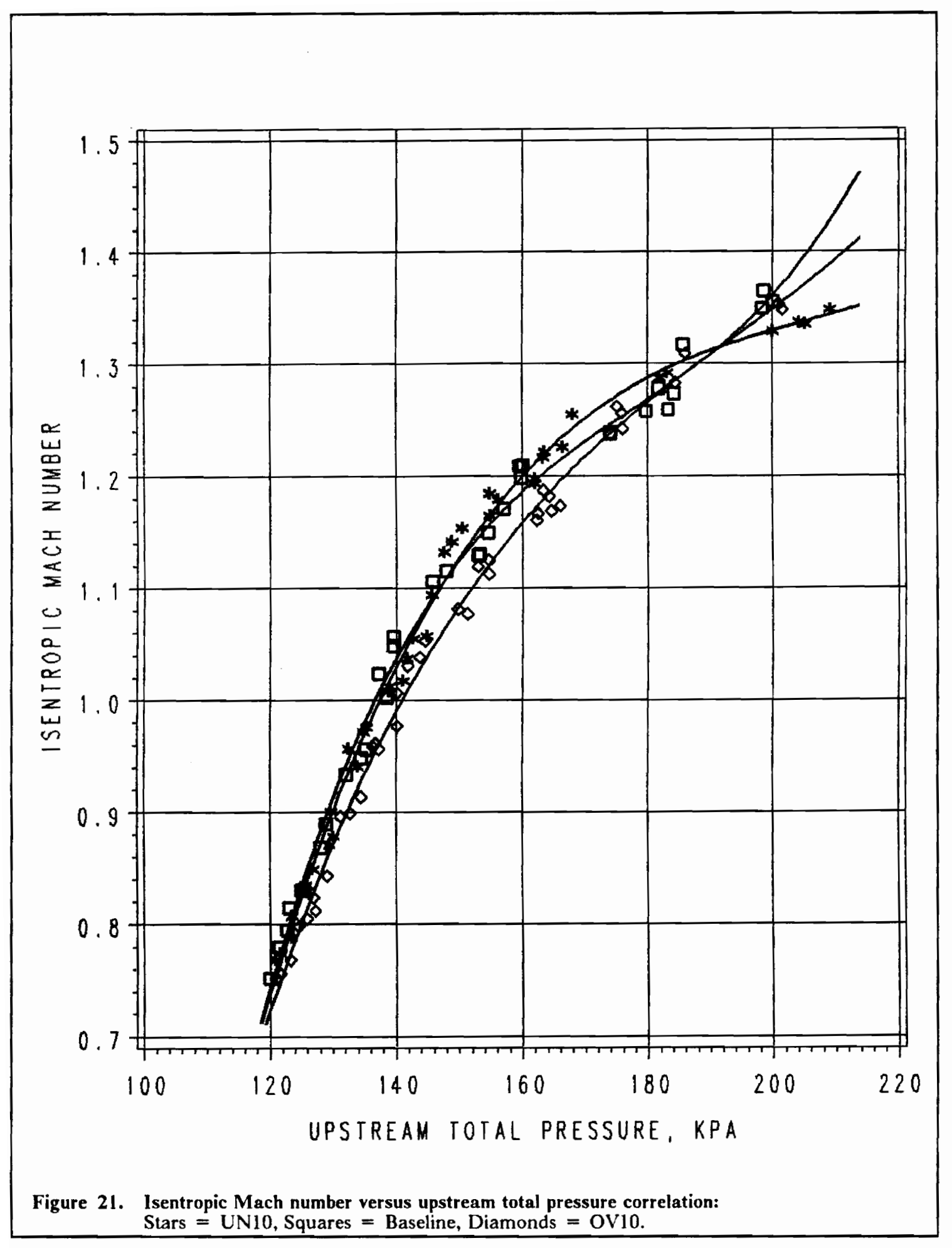




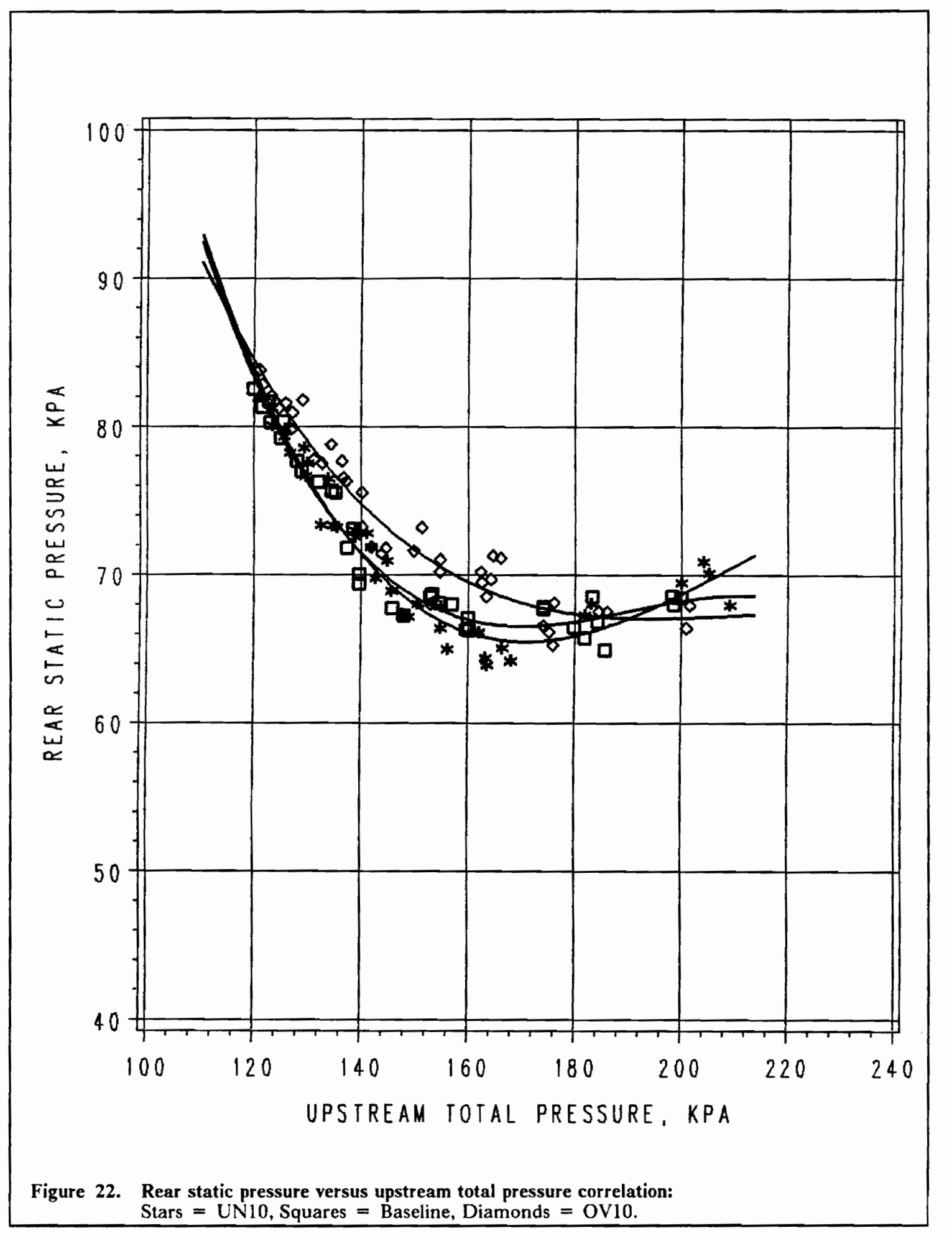




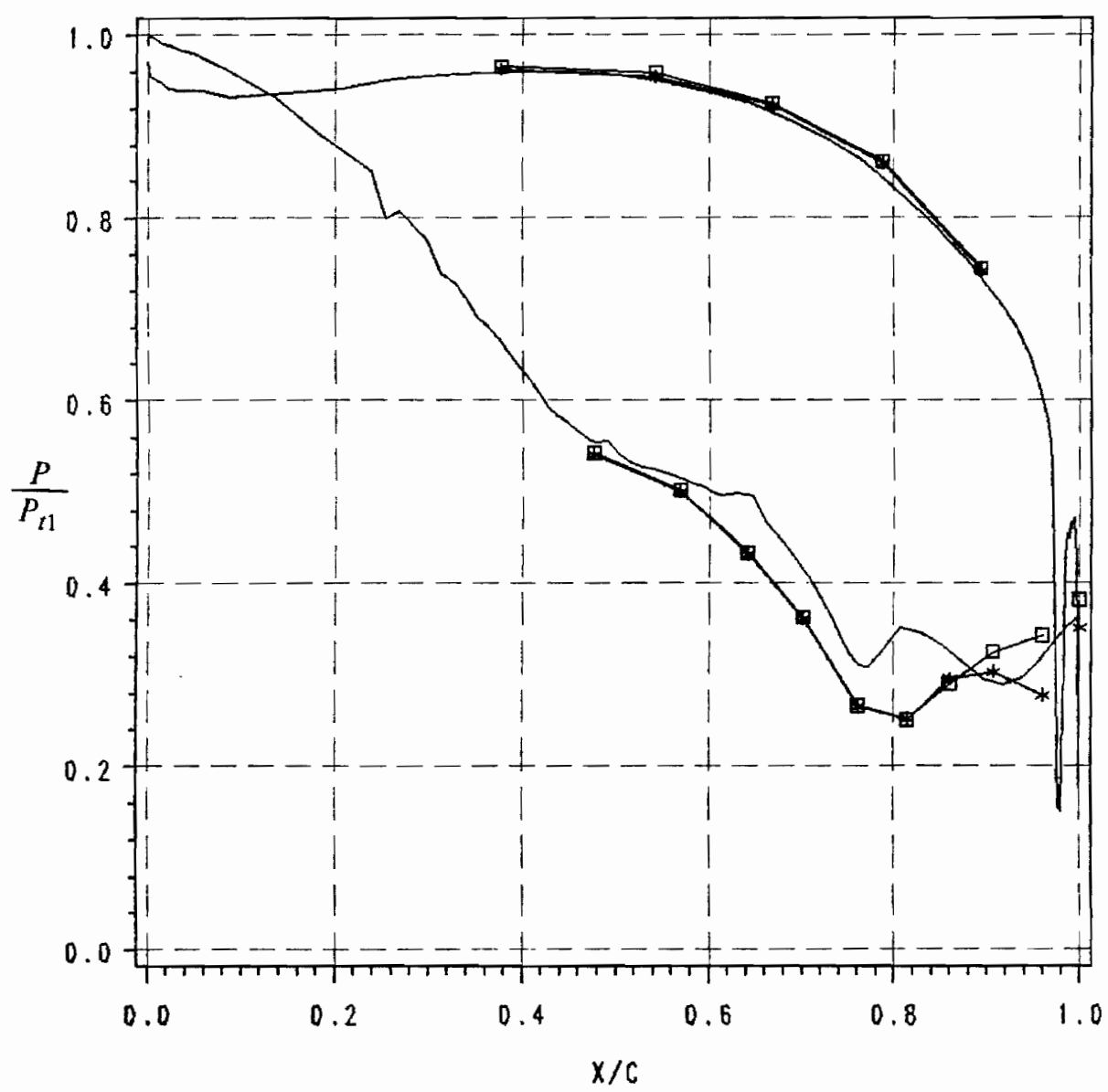

Figure 23. Blade surface static pressure distribution: $-10 \%$ solidity, $\mathbf{P}_{t 1} / \mathbf{P}_{\mathbf{2}}=2.77$ :

Curve: Inviscid solution, $P_{t} / P_{2}=2.77, M_{2 i s}=1.30$

Squares: Experiment, $P_{t 1} / P_{2}=2.56, M_{2 i s}=1.24$

Stars: Experiment, $P_{t 1} \mid P_{2}=2.92, M_{2 i s}=1.34$ 


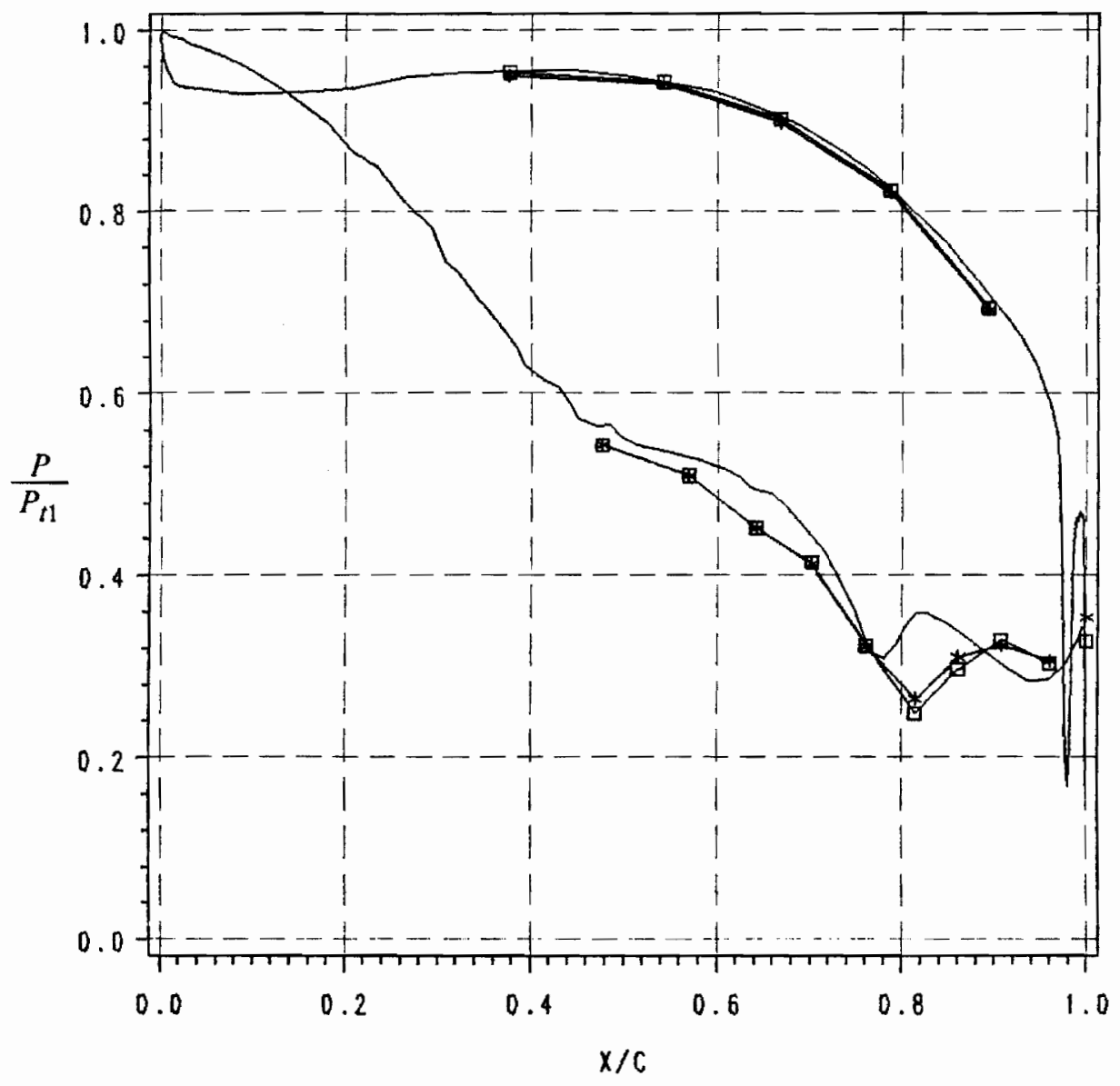

Figure 24. Blade surface static pressure distribution: Baseline solidity, $P_{t 1} / P_{2}=2.78$ :

Curve: Inviscid solution, $P_{t 1} / P_{2}=2.78, M_{2 i s}=1.30$

Squares: Experiment, $P_{t 1} / P_{2}=2.98, M_{2 i s}=1.35$

Stars: Experiment, $P_{t 1} / P_{2}=2.56, M_{2 i s}=1.24$ 


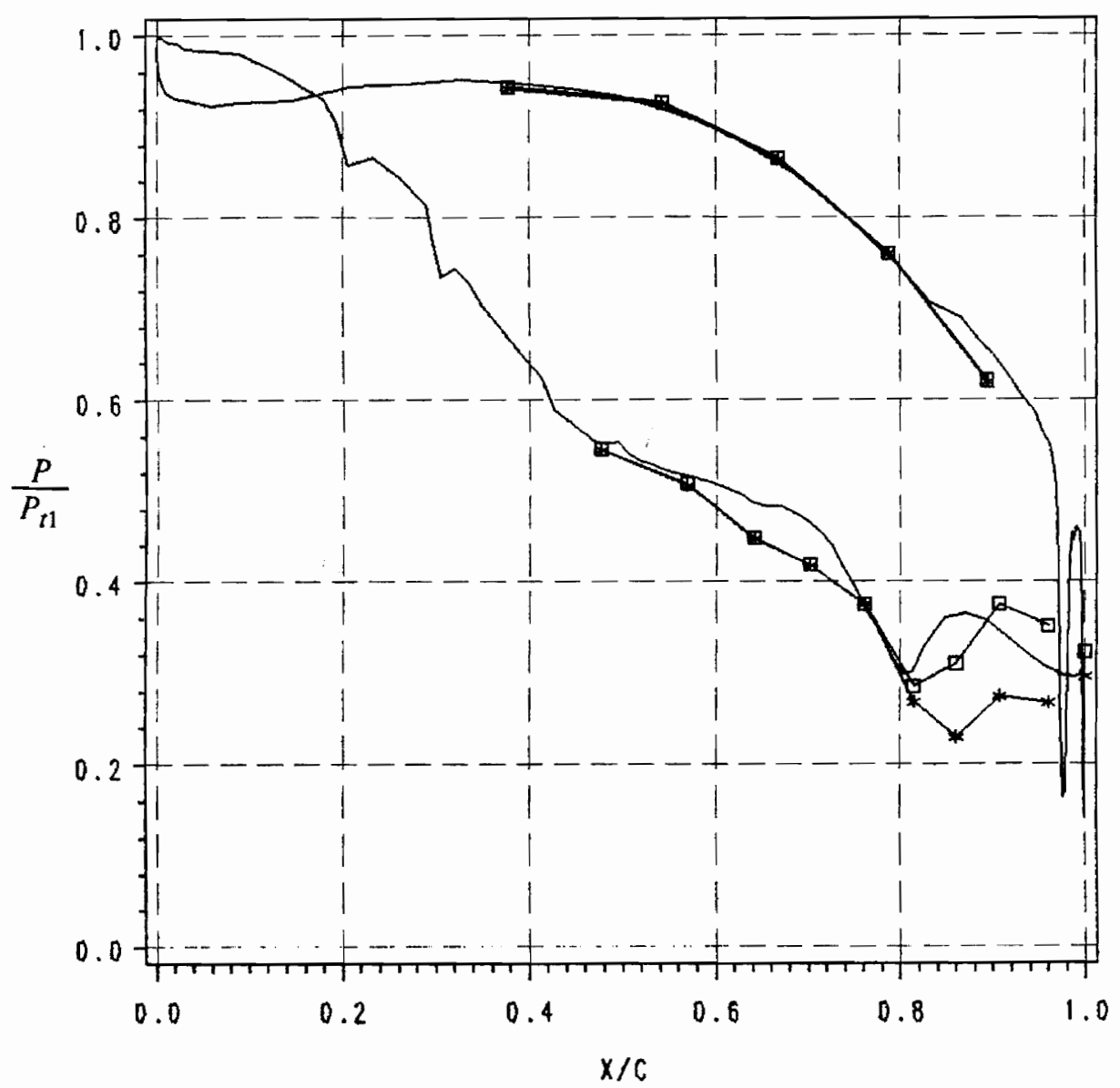

Figure 25. Blade surface static pressure distribution: $+10 \%$ solidity, $P_{t 1} / P_{2}=2.71$ : Curve: Inviscid solution, $P_{t 1} / P_{2}=2.71, M_{2 i s}=1.29$

Squares: Experiment, $P_{t} \mid P_{2}=2.60, M_{2 i s}=1.25$

Stars: Experiment, $P_{t 1} / P_{2}=2.98, M_{2 i s}=1.35$ 


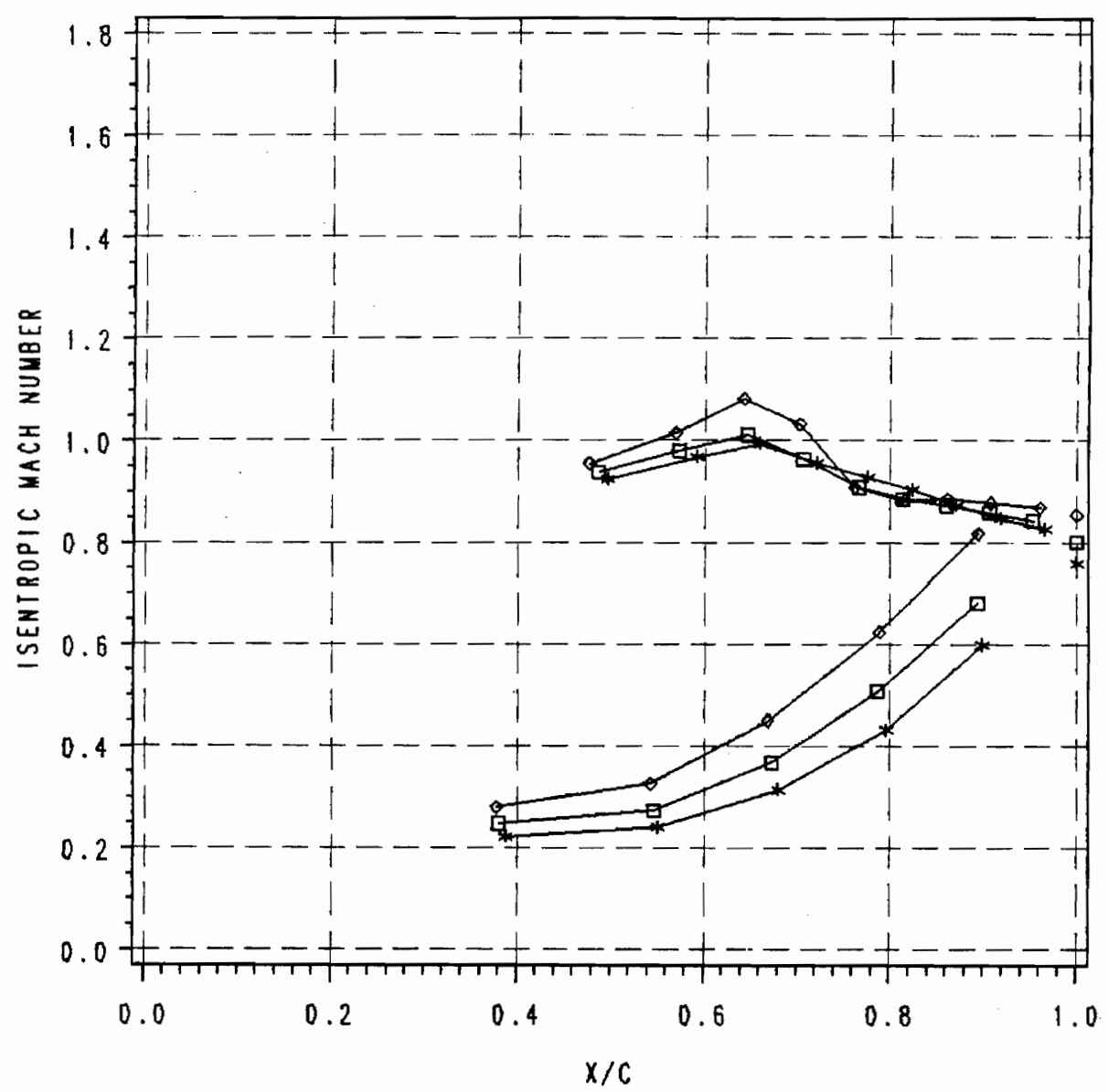

Figure 26. Blade surface Mach number distributions: Three solidities, $\mathbf{M}_{2 i s} \simeq \mathbf{0 . 8 2}$ : Stars: UN10, $\sigma=0.923, M_{2 i s}=0.81$

Squares: Baseline, $\sigma=1.025, M_{2 i s}=0.82$

Diamonds: OV10, $\sigma=1.128, M_{2 i s}=0.84$ 


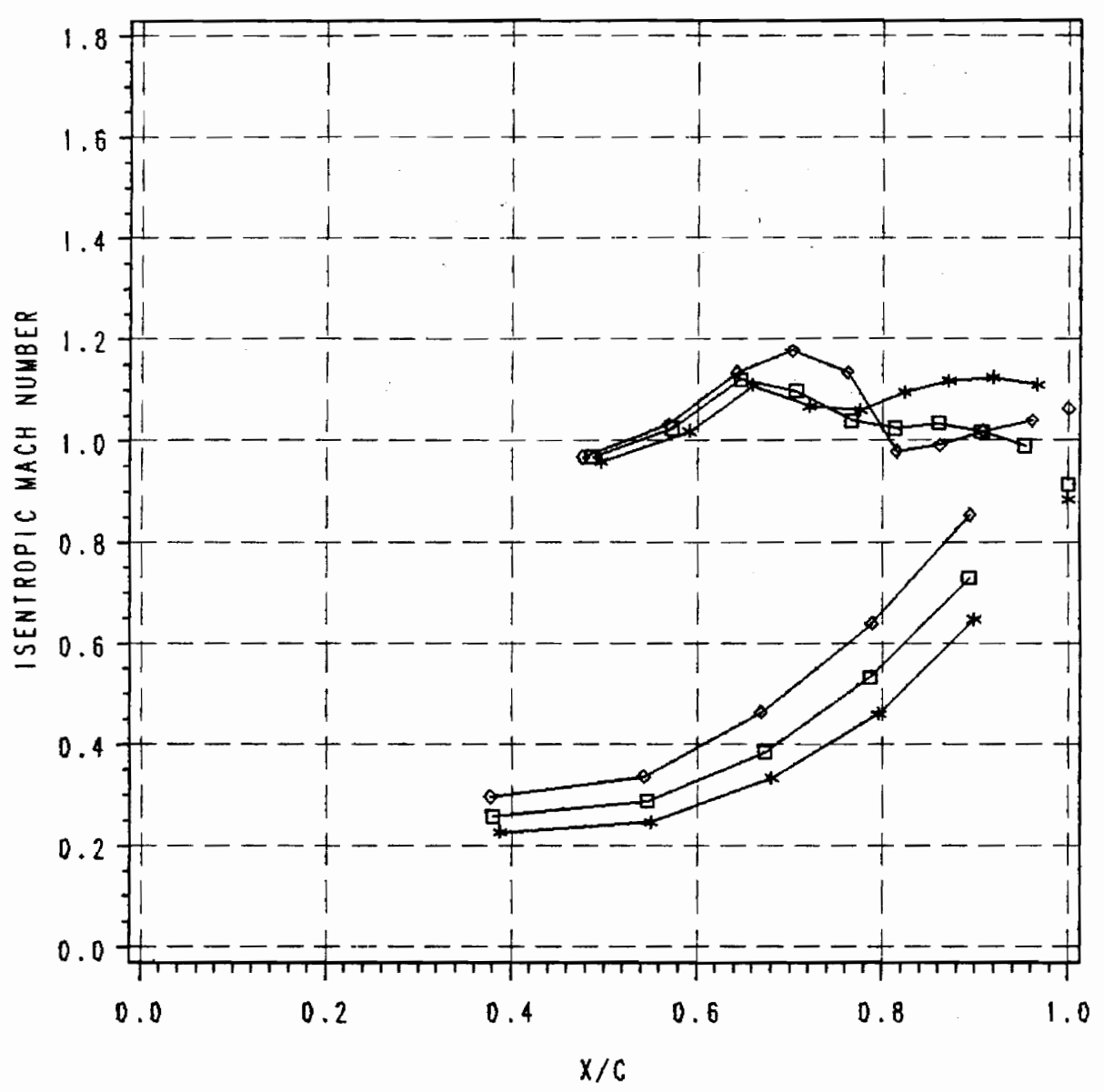

Figure 27. Blade surface Mach number distributions: Three solidities, $\mathbf{M}_{2 i s} \simeq \mathbf{0 . 9 7}$ : Stars: UN10, $\sigma=0.923, M_{2 i s}=0.97$

Squares: Baseline, $\sigma=1.025, M_{2 i s}=0.95$

Diamonds: OV10, $\sigma=1.128, M_{2 i s}=0.97$ 


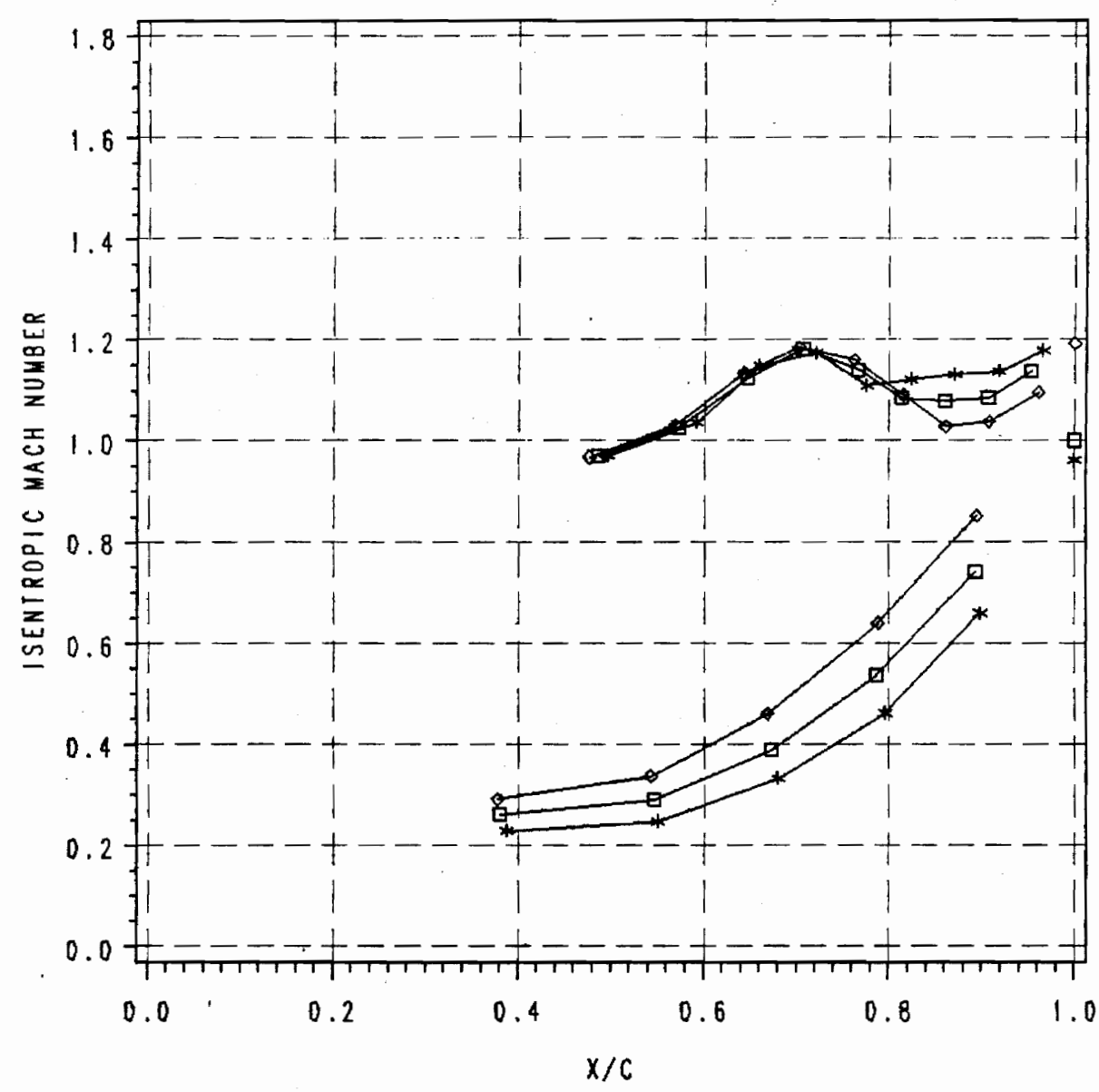

Figure 28. Blade surface Mach number distributions: Three solidities, $\mathbf{M}_{2 i s} \simeq 1.03$ : Stars: UN10, $\sigma=0.923, M_{2 i s}=1.03$

Squares: Baseline, $\sigma=1.025, M_{2 i s}=1.03$

Diamonds: OV10, $\sigma=1.128, M_{2 i s}=1.01$ 


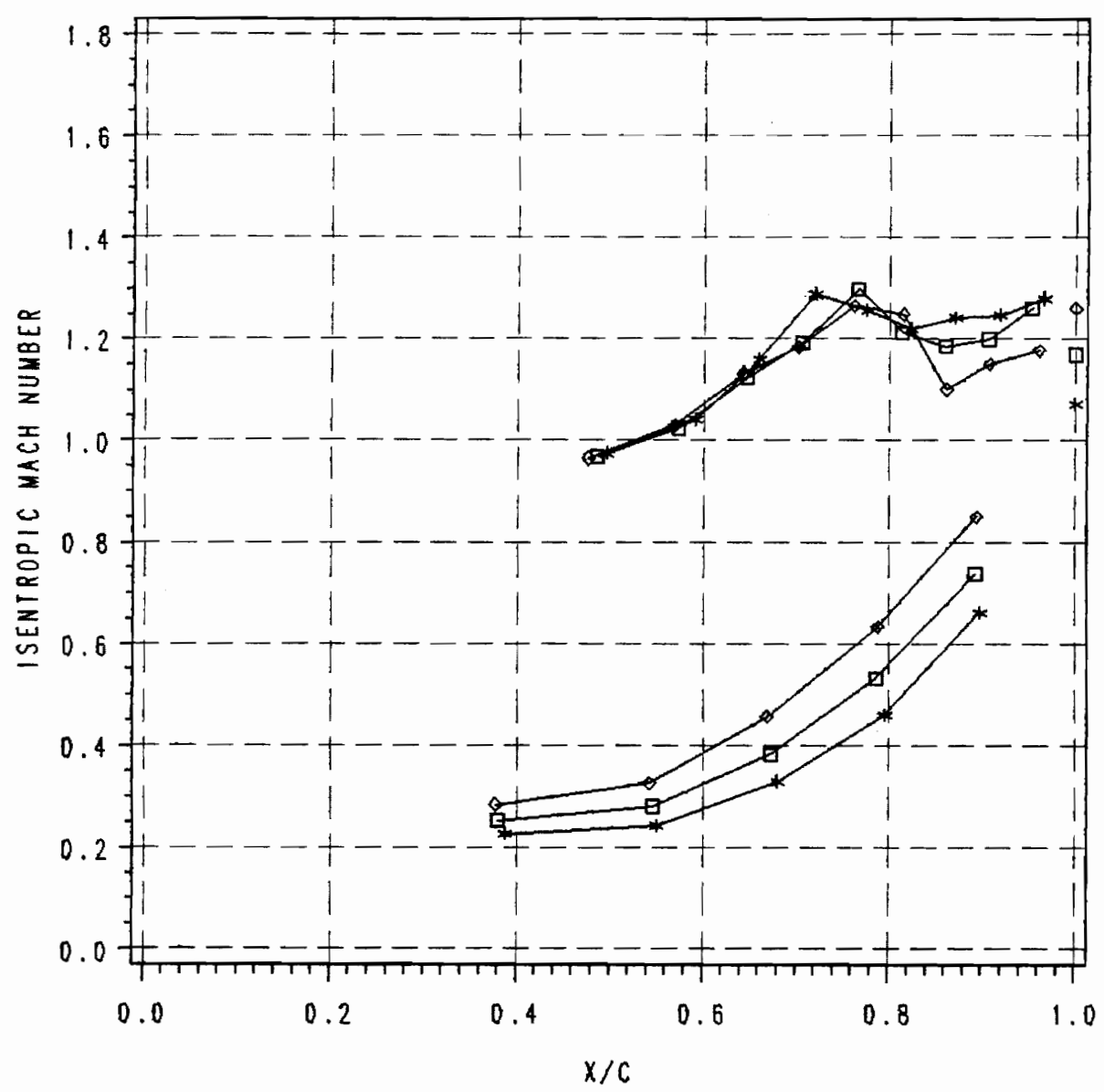

Figure 29. Blade surface Mach number distributions: Three solidities, $\mathbf{M}_{2 i s} \simeq 1.11$ : Stars: UN10, $\sigma=0.923, M_{2 i s}=1.12$

Squares: Baseline, $\sigma=1.025, M_{2 i s}=1.11$

Diamonds: OV10, $\sigma=1.128, M_{2 i s}=1.10$ 


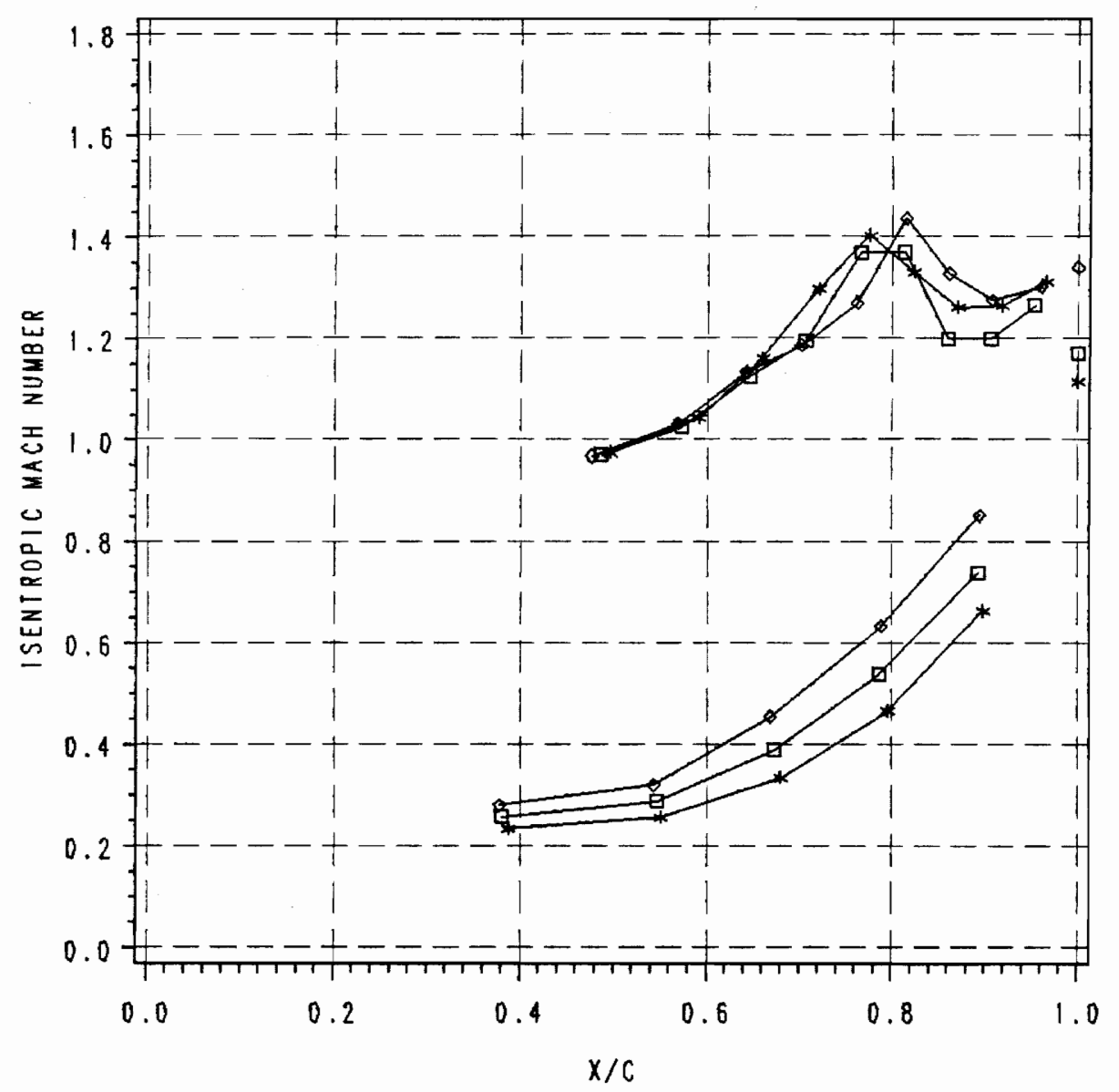

Figure 30. Blade surface Mach number distributions: Three solidities, $\mathbf{M}_{2 i s} \simeq 1.19$ : Stars: UN10, $\sigma=0.923, M_{2 i s}=1.17$

Squares: Baseline, $\sigma=1.025, M_{2 i s}=1.19$

Diamonds: OV10, $\sigma=1.128, M_{2 i s}=1.20$ 


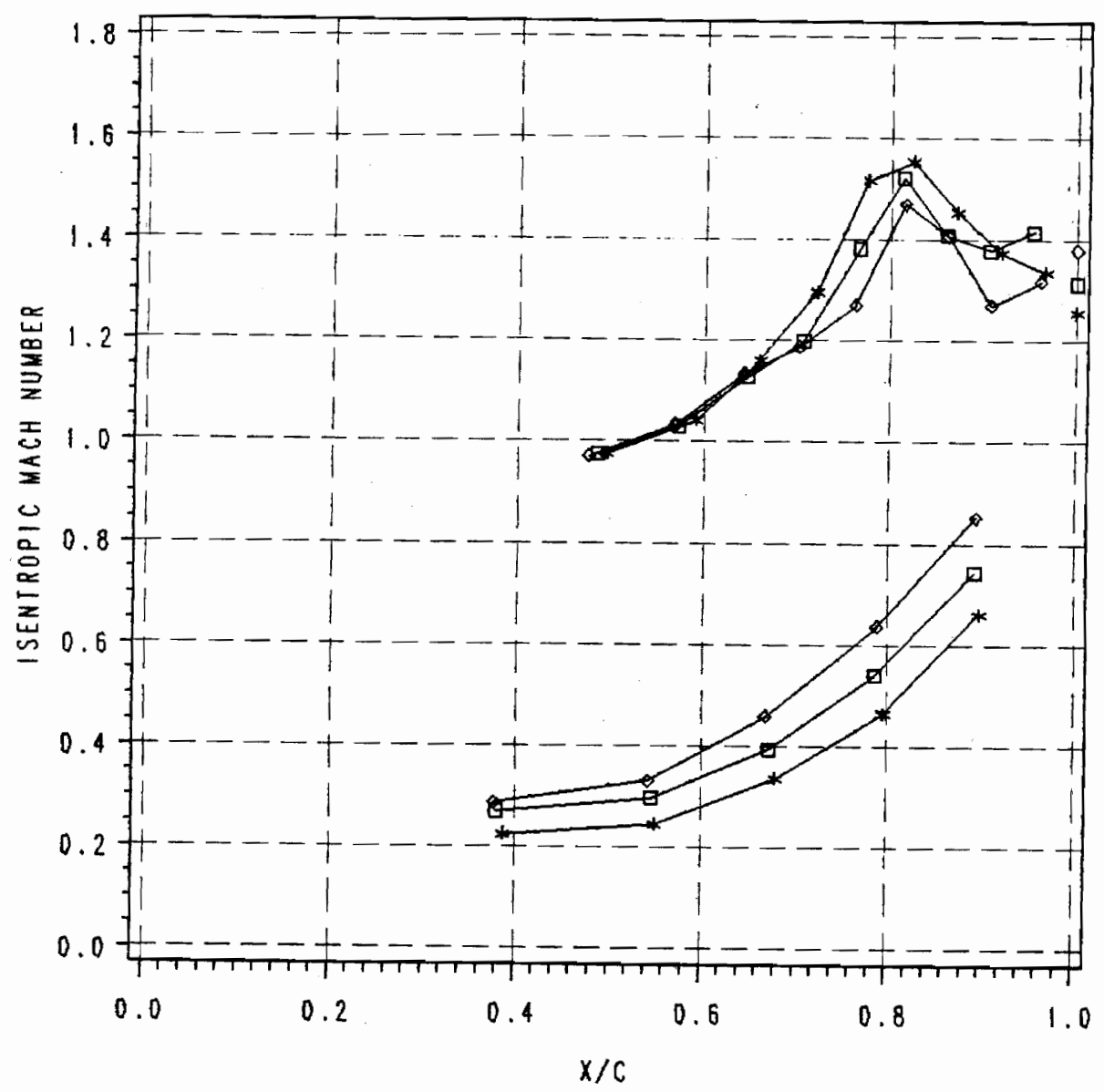

Figure 31. Blade surface Mach number distributions: Three solidities, $\mathbf{M}_{2 \text { is }} \simeq$ 1.24: Stars: UN10, $\sigma=0.923, M_{2 i s}=1.24$

Squares: Baseline, $\sigma=1.025, M_{2 i s}=1.24$

Diamonds: $\mathrm{OV} 10, \sigma=1.128, M_{2 i s}=1.25$ 


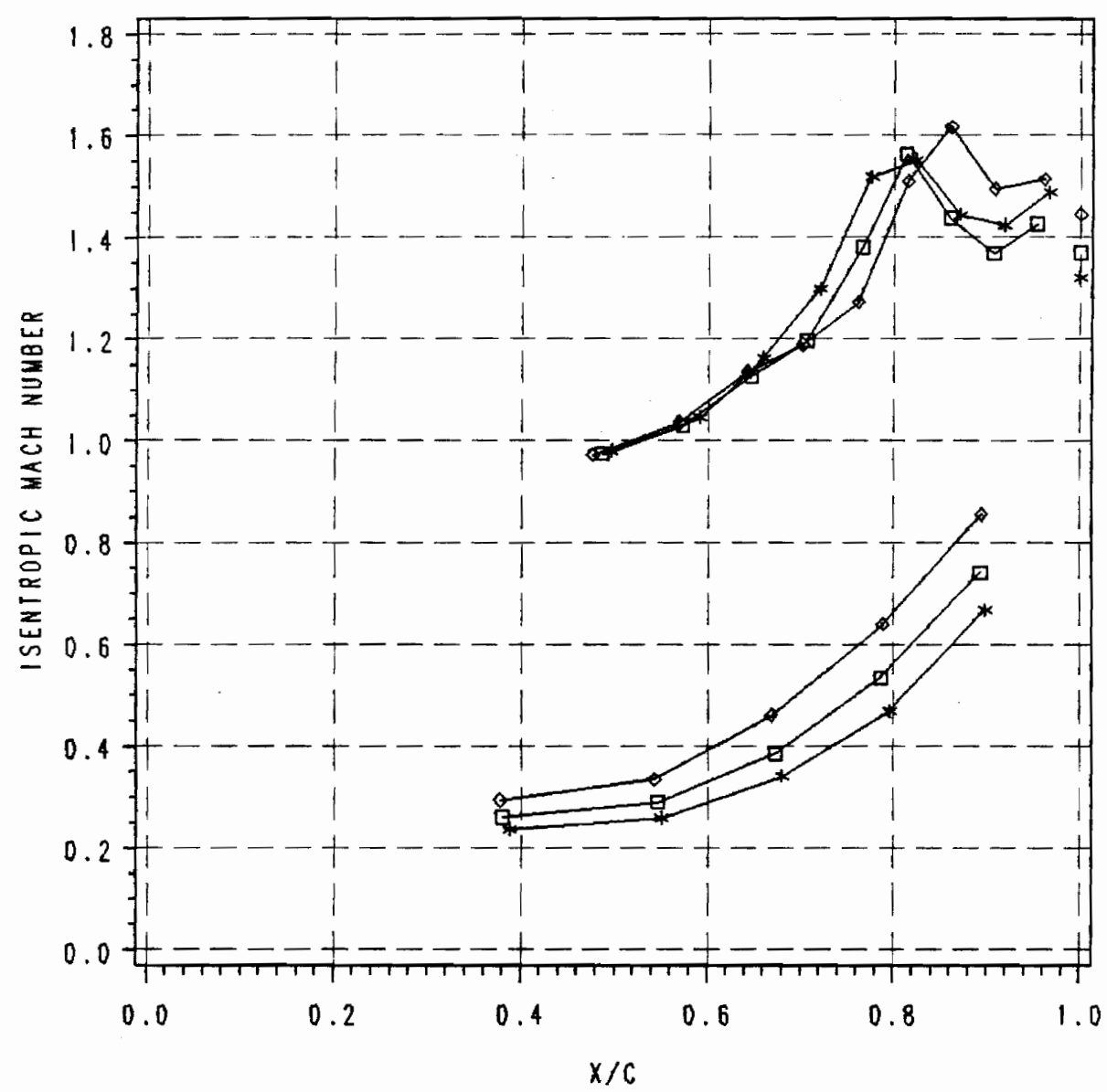

Figure 32. Blade surface Mach number distributions: Three solidities, $\mathbf{M}_{2 i s} \simeq 1.35$ : Stars: UN10, $\sigma=0.923, M_{2 i s}=1.34$

Squares: Baseline, $\sigma=1.025, M_{2 i s}=1.35$

Diamonds: OV10, $\sigma=1.128, M_{2 i s}=1.35$ 


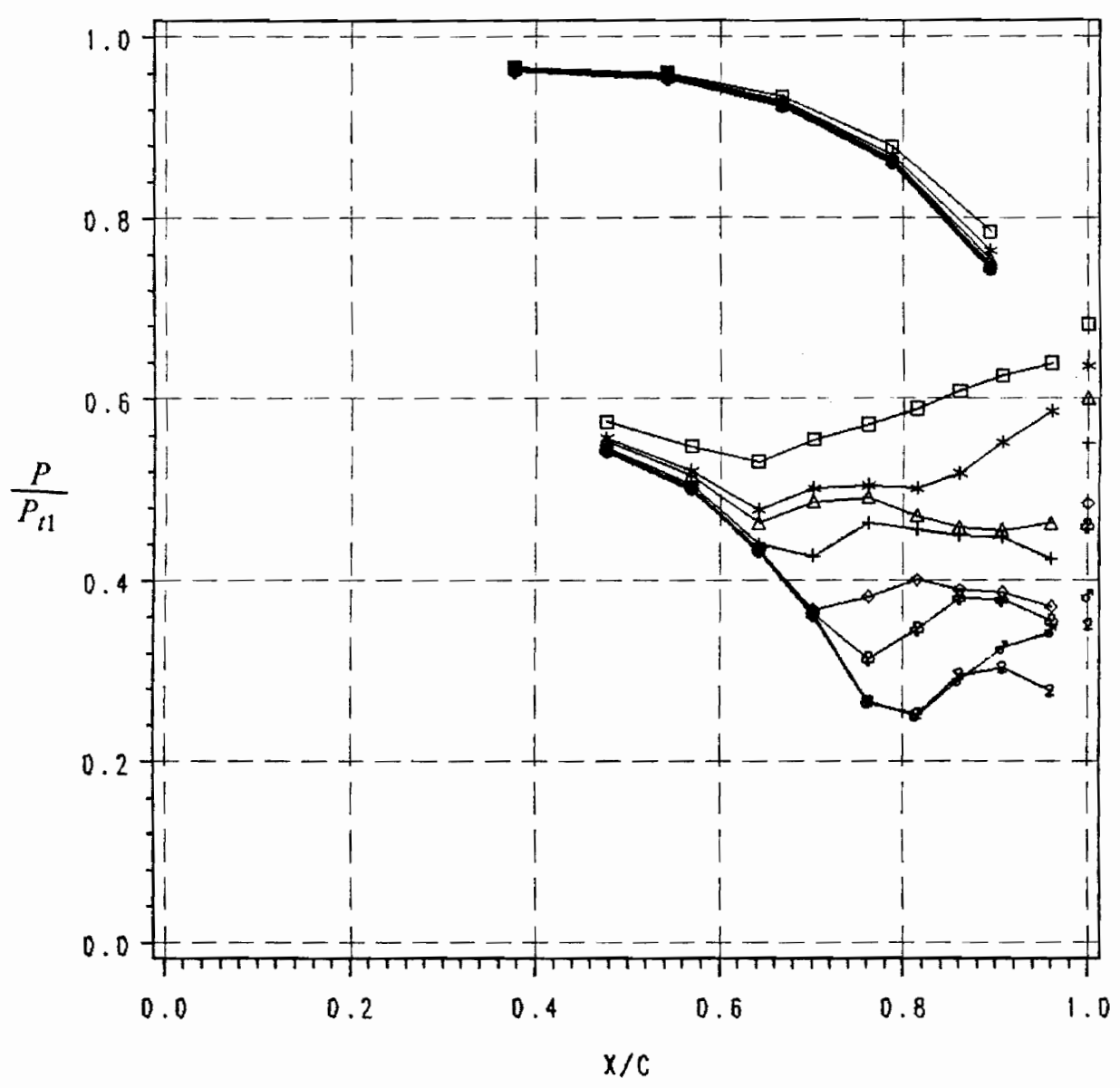

Figure 33. Blade surface static pressure distributions: $U N 10$ cascade, $\sigma=0.923$ : Square: $M_{2 i s}=0.81, P_{t 1} / P_{2}=1.54$

Star: $M_{2 i s}=0.89, P_{t 1} / P_{2}=1.68$

Triangle: $M_{2 i s}=0.97, P_{t 1} / P_{2}=1.83$

Plus: $M_{2 i s}=1.03, P_{t 1} / P_{2}=1.92$

Diamond: $M_{2 i s}=1.12, P_{t 1} / P_{2}=2.18$

Club: $M_{2 i s}=1.17, P_{t i} \mid P_{2}=2.34$

Male: $M_{2 i s}=1.24, P_{t 1} / P_{2}=2.56$

Female: $M_{2 i s}=1.34, P_{t 1} / P_{2}=2.92$ 


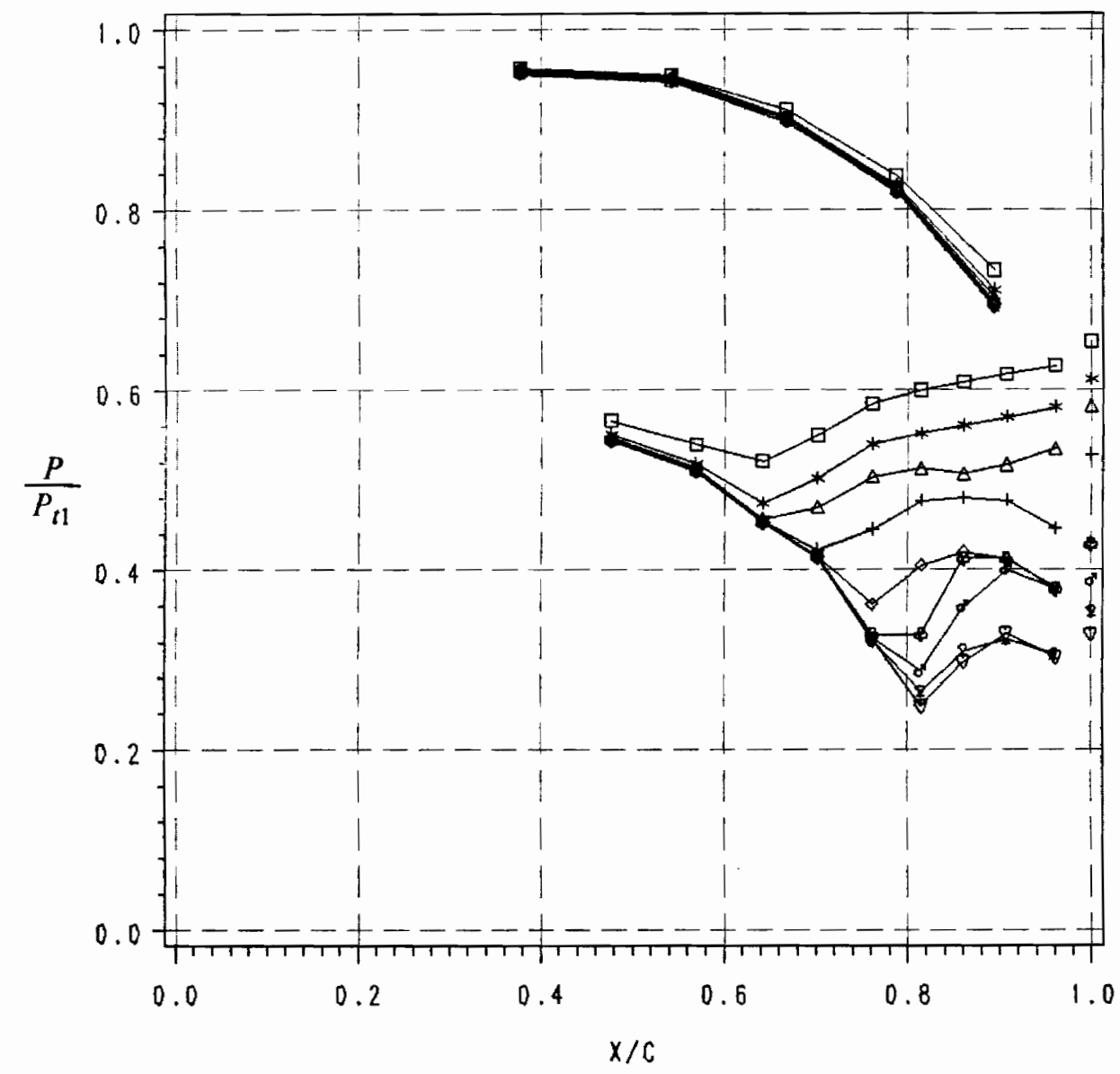

Figure 34. Blade surface static pressure distributions: Baseline cascade, $\sigma=1.025$ :

Square: $M_{2 i s}=0.82, P_{t 1} / P_{2}=1.55$

Star: $M_{2 i s}=0.89, P_{t 1} \mid P_{2}=1.66$

Triangle: $M_{2 l s}=0.95, P_{t 1} / P_{2}=1.79$

Plus: $M_{2 i s}=1.03, P_{n 1} / P_{2}=1.95$

Diamond: $M_{2 i s}=1.11, P_{t 1} / P_{2}=2.16$

Club: $M_{2 i s}=1.19, P_{t 1} / P_{2}=2.41$

Male: $M_{2 i s}=1.21, P_{\mathrm{t} 1} / P_{2}=2.45$

Female: $M_{2 i s}=1.24, P_{t 1} / P_{2}=2.56$

Heart: $M_{2 i s}=1.35, P_{t 1} / P_{2}=2.98$ 


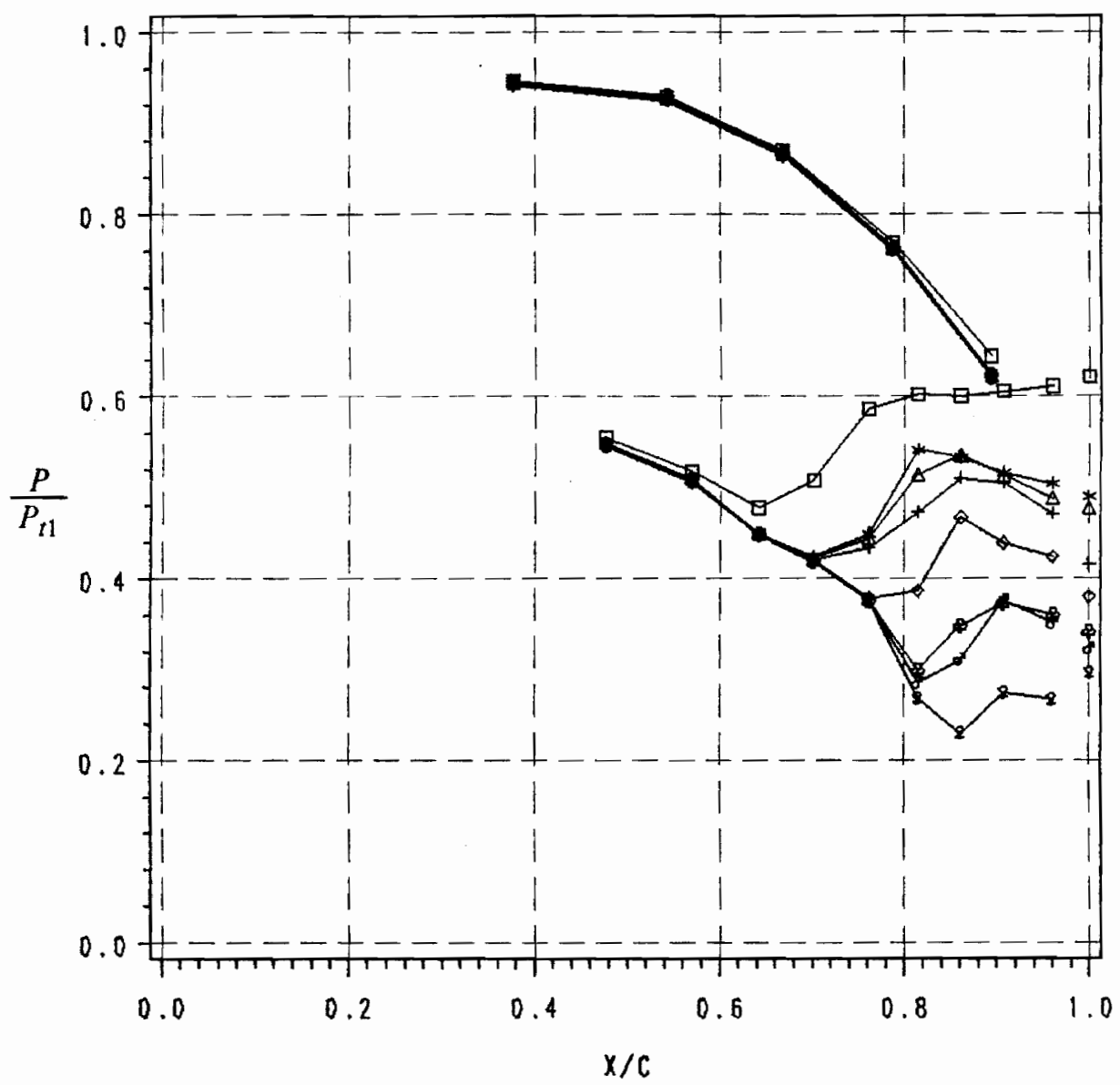

Figure 35. Blade surface static pressure distributions: $O$ V10 cascade, $\sigma=1.128$ :

Square: $M_{2 i s}=0.84, P_{t i} / P_{2}=1.59$

Star: $M_{2 i s}=0.97, P_{t 1} / P_{2}=1.82$

Triangle: $M_{2 i s}=0.98, P_{11} / P_{2}=1.85$

Plus: $M_{2 i s}=1.01, P_{t 1} / P_{2}=1.92$

Diamond: $M_{2 i s}=1.10, P_{t} \mid P_{2}=2.12$

Club: $M_{2 i s}=1.20, P_{t 1} / P_{2}=2.42$

Male: $M_{2 i s}=1.25, P_{t 1} \mid P_{2}=2.60$

Female: $M_{2 i s}=1.35, P_{t 1} / P_{2}=2.98$ 


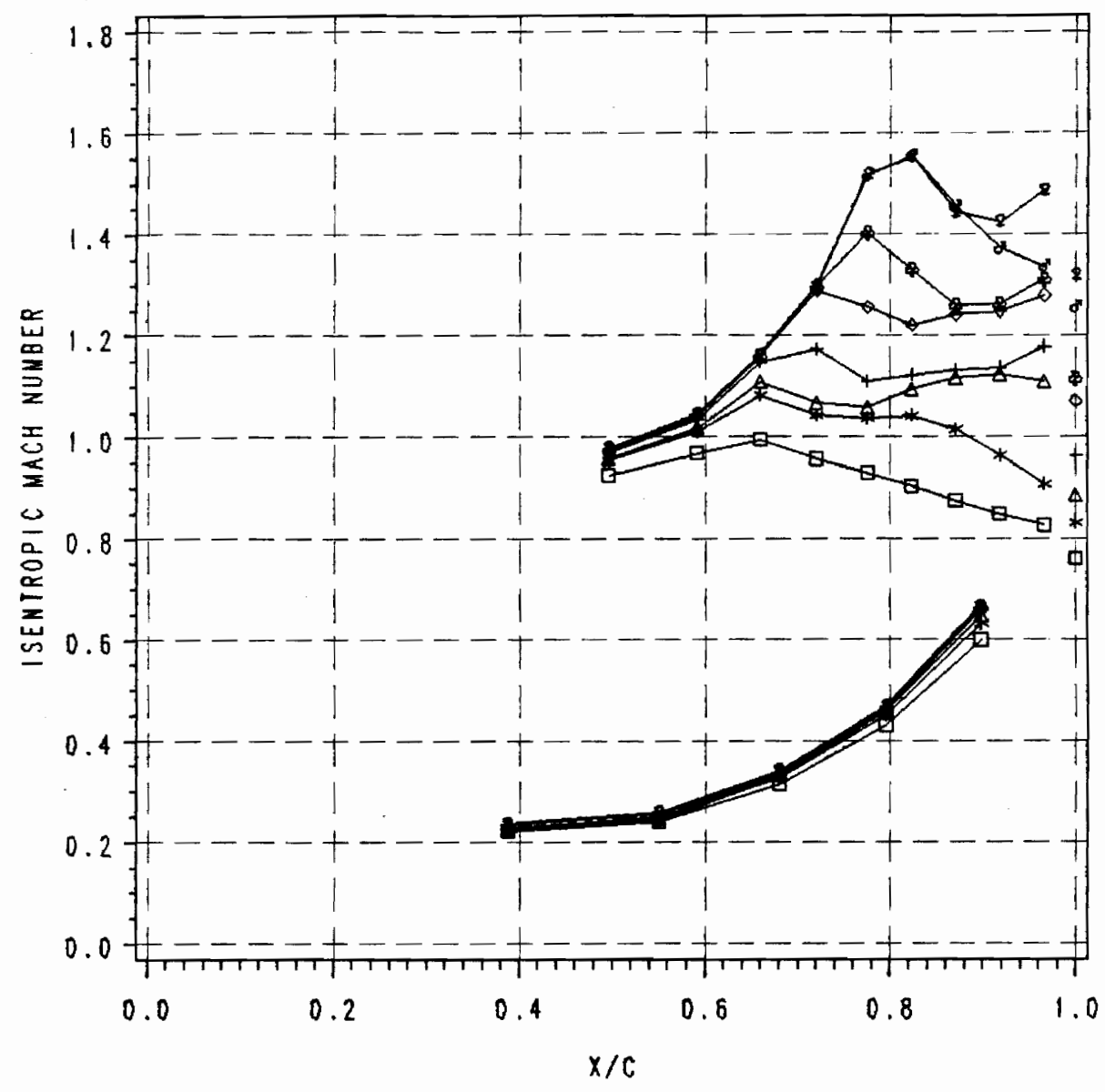

Figure 36. Blade surface Mach number distributions: $U N 10$ cascade, $\sigma=0.923$ :

Square: $M_{2 i s}=0.81, P_{t 1} / P_{2}=1.54$

Star: $M_{2 i s}=0.89, P_{t 1} / P_{2}=1.68$

Triangle: $M_{2 i s}=0.97, P_{t 1} / P_{2}=1.83$

Plus: $M_{2 i s}=1.03, P_{t 1} / P_{2}=1.92$

Diamond: $M_{2 i s}=1.12, P_{t 1} / P_{2}=2.18$

Club: $M_{2 i s}=1.17, P_{t 1} / P_{2}=2.34$

Male: $M_{2 i s}=1.24, P_{t 1} / P_{2}=2.56$

Female: $M_{2 i s}=1.34, P_{t 1} / P_{2}=2.92$ 


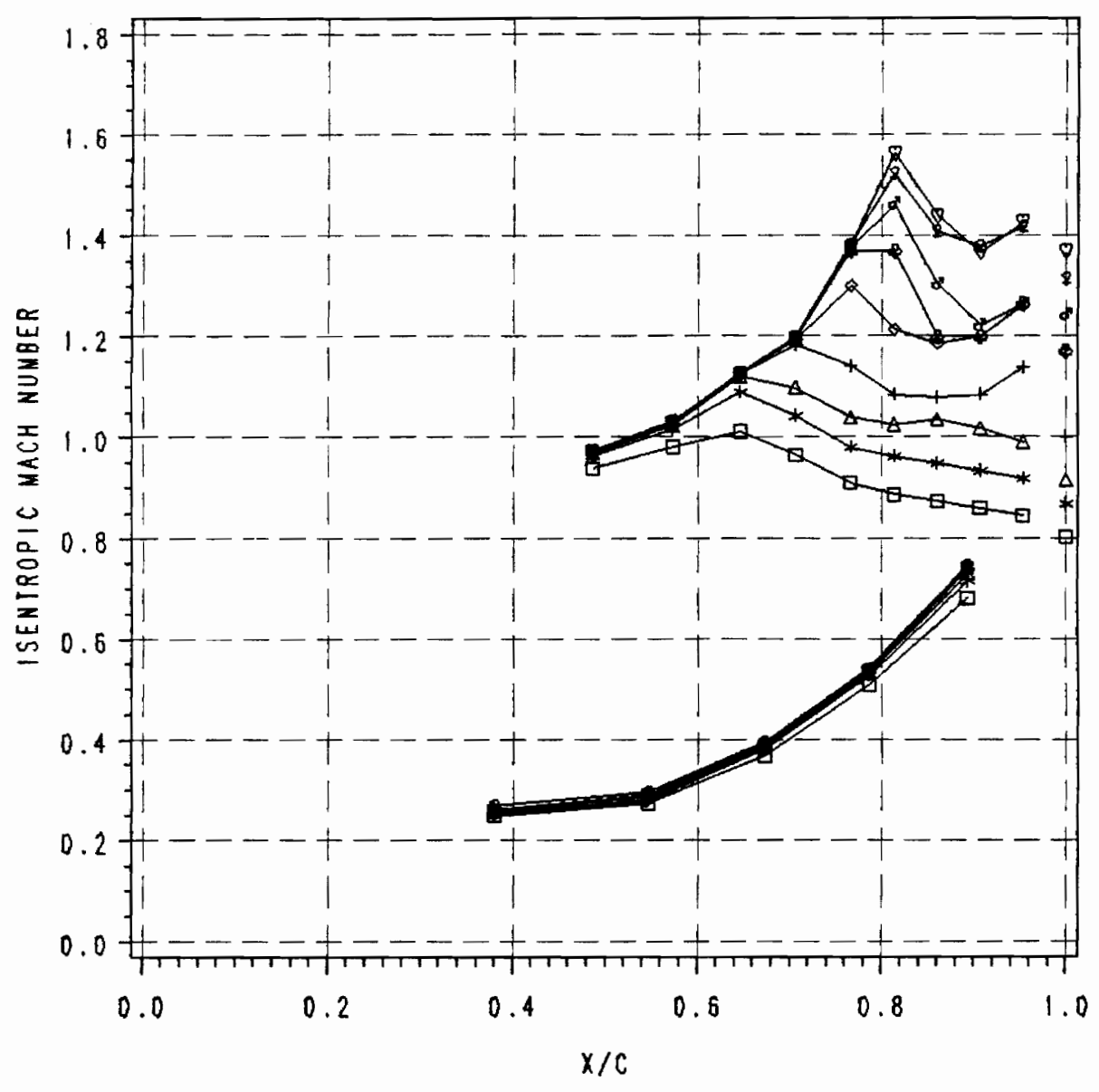

Figure 37. Blade surface Mach number distributions: Baseline cascade, $\sigma=1.025$ :

Square: $M_{2 i s}=0.82, P_{t 1} / P_{2}=1.55$

Star: $M_{2 i s}=0.89, P_{t 1} \mid P_{2}=1.66$

Triangle: $M_{2 i s}=0.95, P_{t 1} / P_{2}=1.79$

Plus: $M_{2 i s}=1.03, P_{t 1} / P_{2}=1.95$

Diamond: $M_{2 i s}=1.11, P_{t 1} \mid P_{2}=2.16$

Club: $M_{2 i s}=1.19, P_{t 1} / P_{2}=2.41$

Male: $M_{2 i s}=1.21, P_{i 1} / P_{2}=2.45$

Female: $M_{2 i s}=1.24, P_{t 1} / P_{2}=2.56$

Heart: $M_{2 i s}=1.35, P_{t 1} / P_{2}=2.98$ 


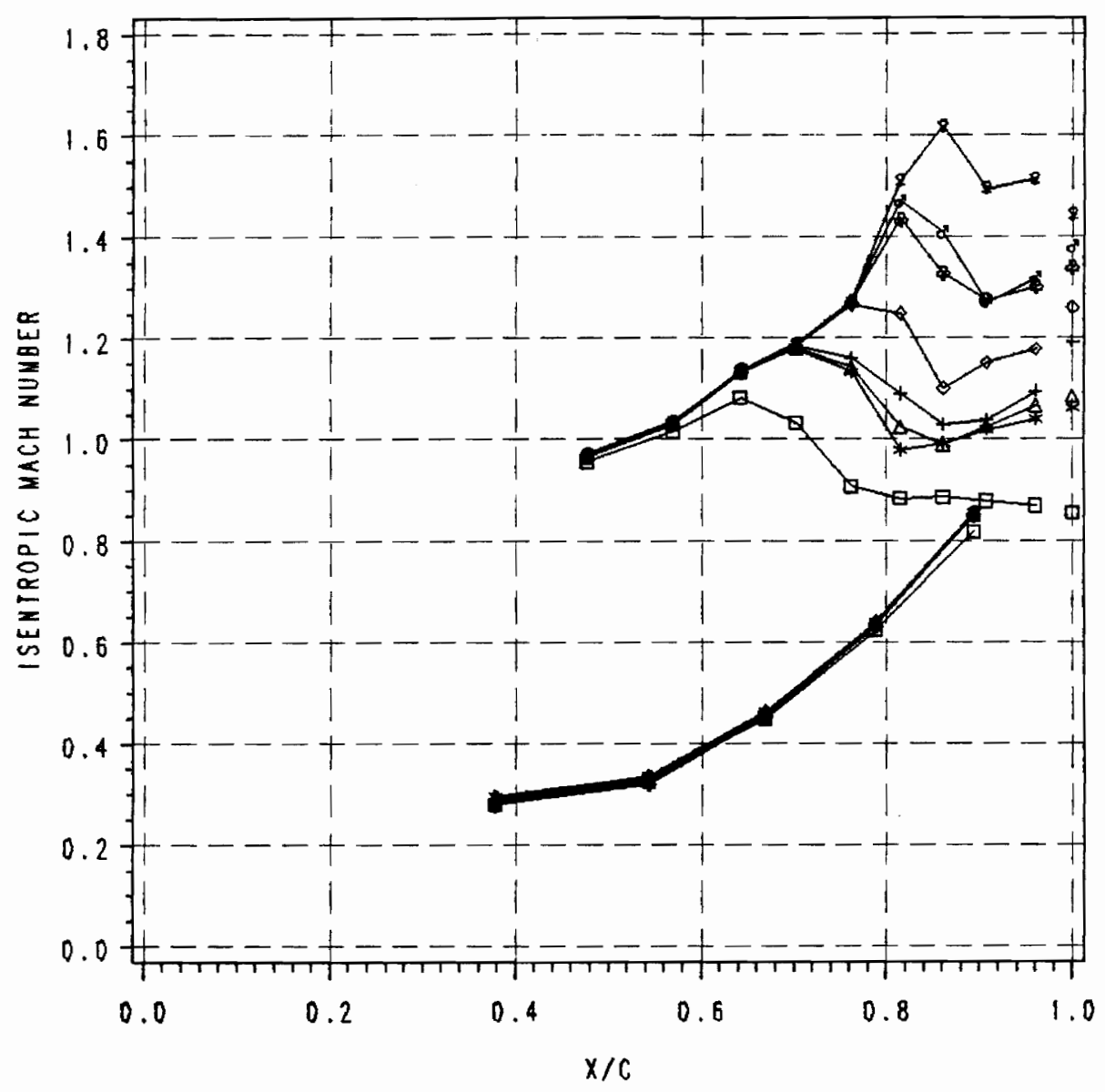

Figure 38. Blade surface Mach number distributions: $O V 10$ cascade, $\sigma=1.128$ :

Square: $M_{2 i s}=0.84, P_{t 1} / P_{2}=1.59$

Star: $M_{2 i s}=0.97, P_{t 1} / P_{2}=1.82$

Triangle: $M_{2 i s}=0.98, P_{t 1} / P_{2}=1.85$

Plus: $M_{2 i s}=1.01, P_{t 1} \mid P_{2}=1.92$

Diamond: $M_{2 i s}=1.10, P_{t 1} / P_{2}=2.12$

Club: $M_{2 i s}=1.20, P_{t 1} / P_{2}=2.42$

Male: $M_{2 i s}=1.25, P_{t 1} / P_{2}=2.60$

Female: $M_{2 i s}=1.35, P_{t 1} / P_{2}=2.98$ 


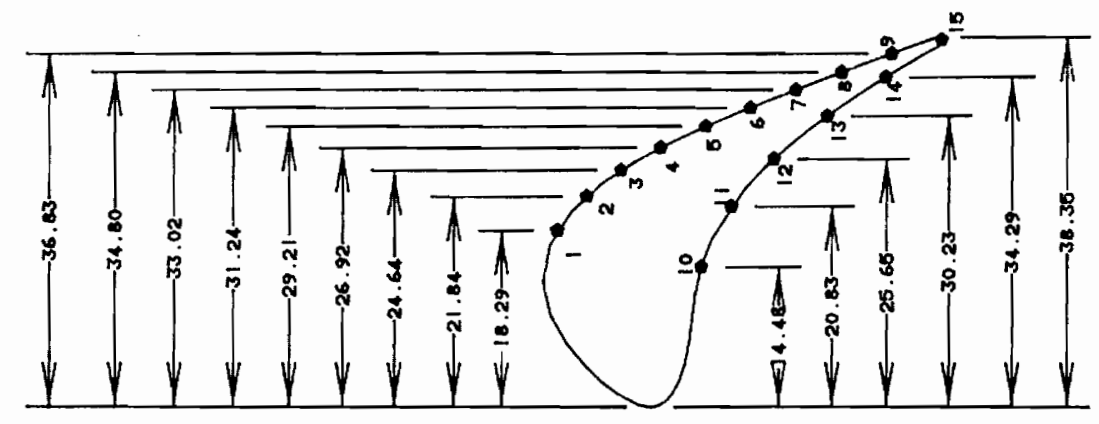

3

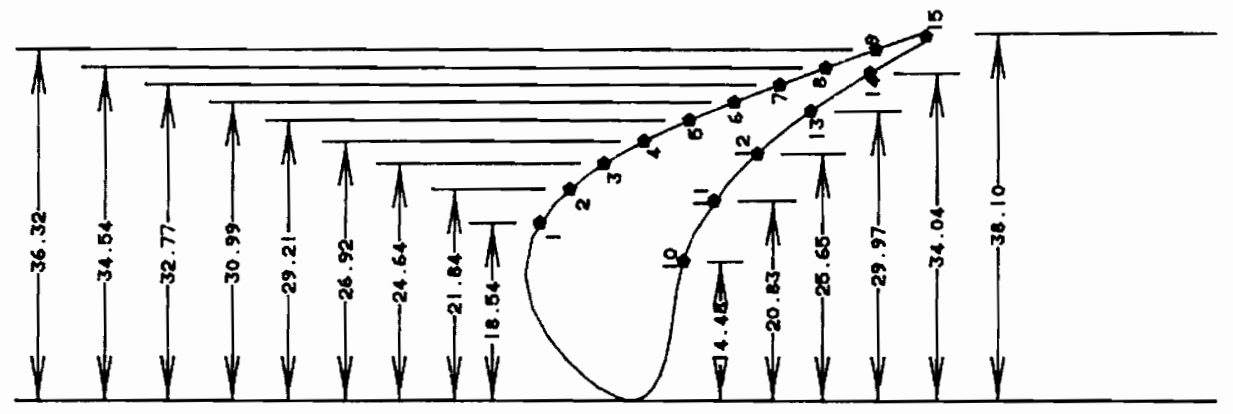

$\frac{1}{4}$

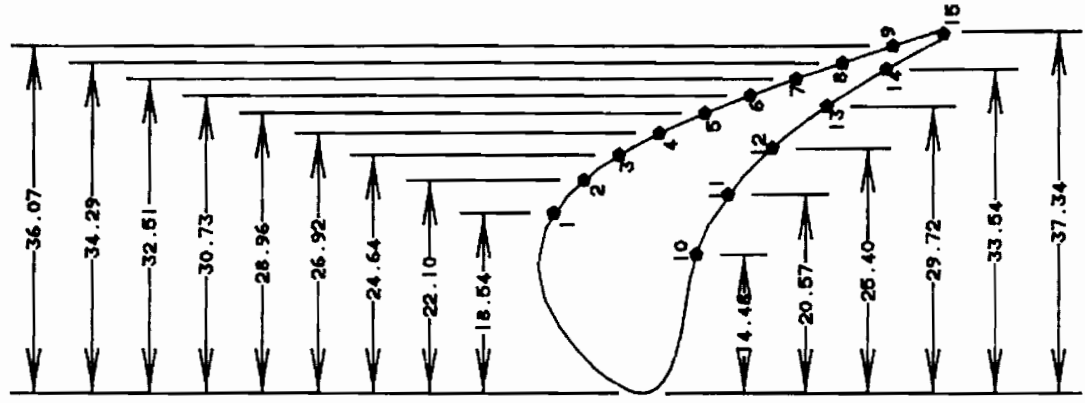

$\frac{0}{3}$

Figure 39. Location of blade static pressure taps: Dimensions in $\mathrm{mm}$. 


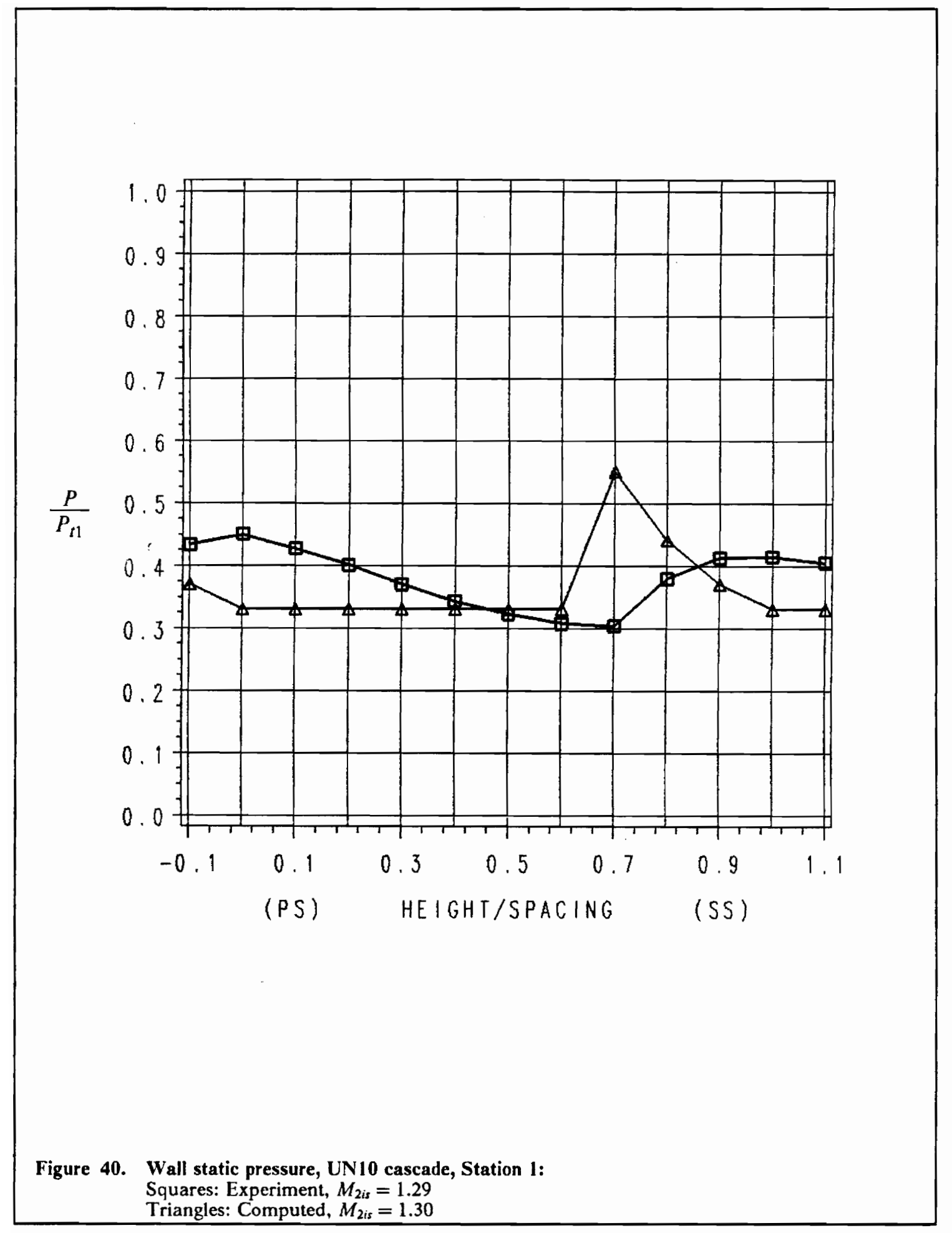




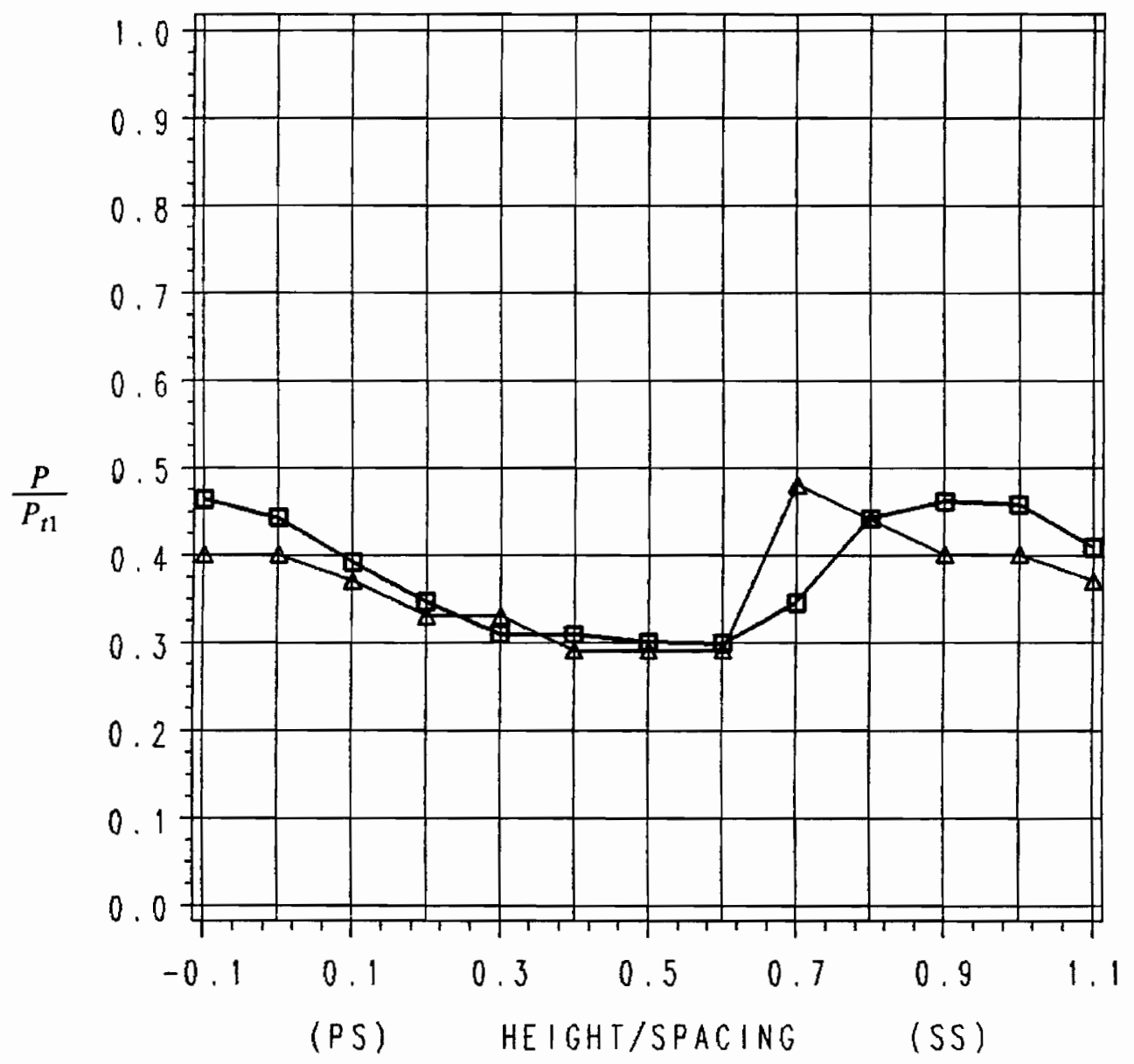

Figure 41. Wall static pressure, UN10 cascade, Station 2:

Squares: Experiment, $M_{2 i s}=1.29$

Triangles: Computed, $M_{2 i s}=1.30$ 


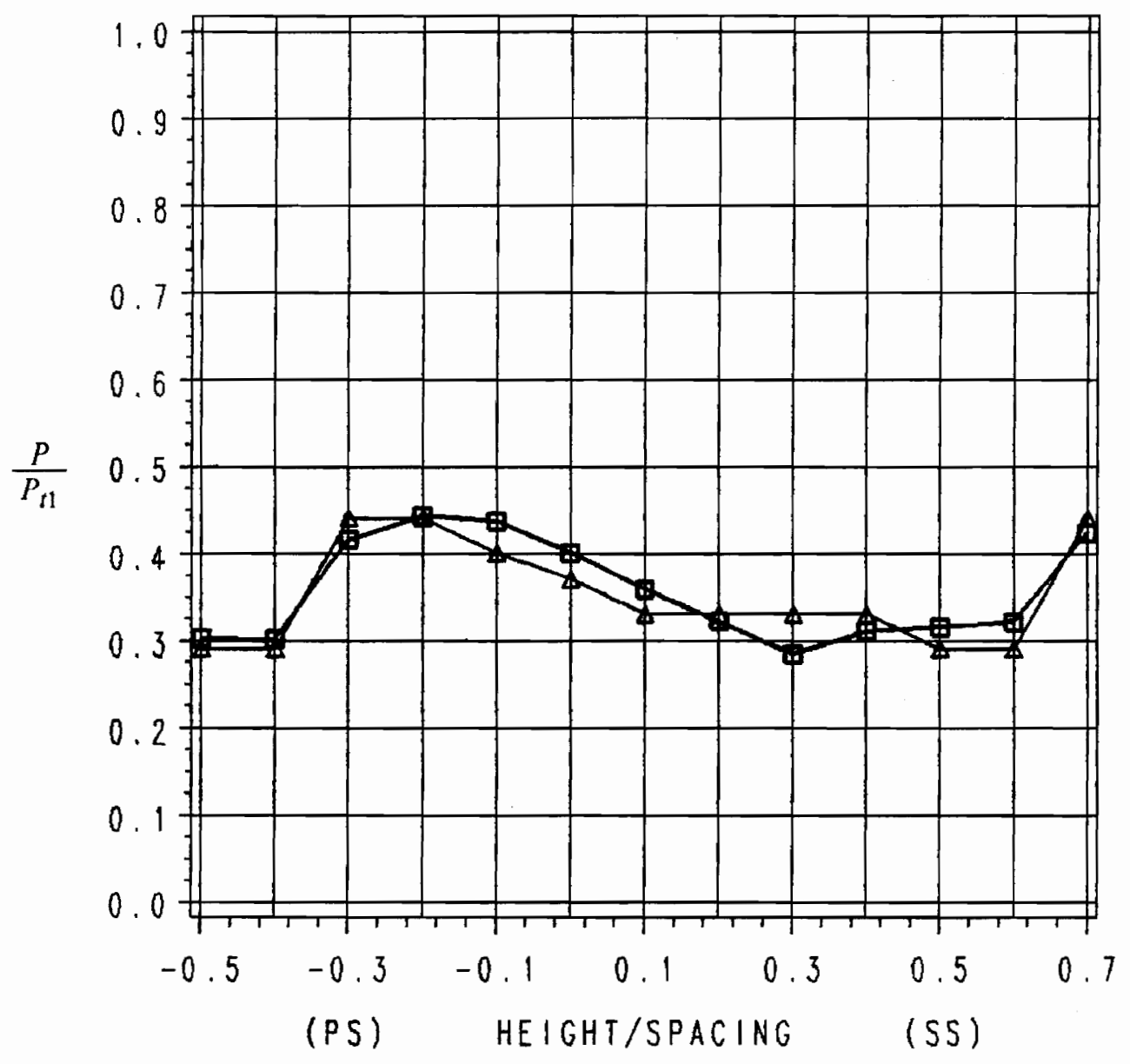

Figure 42. Wall static pressure, Baseline cascade, Station 1:

Squares: Experiment, $M_{2 i s}=1.30$

Triangles: Computed, $M_{2 i s}=1.30$ 


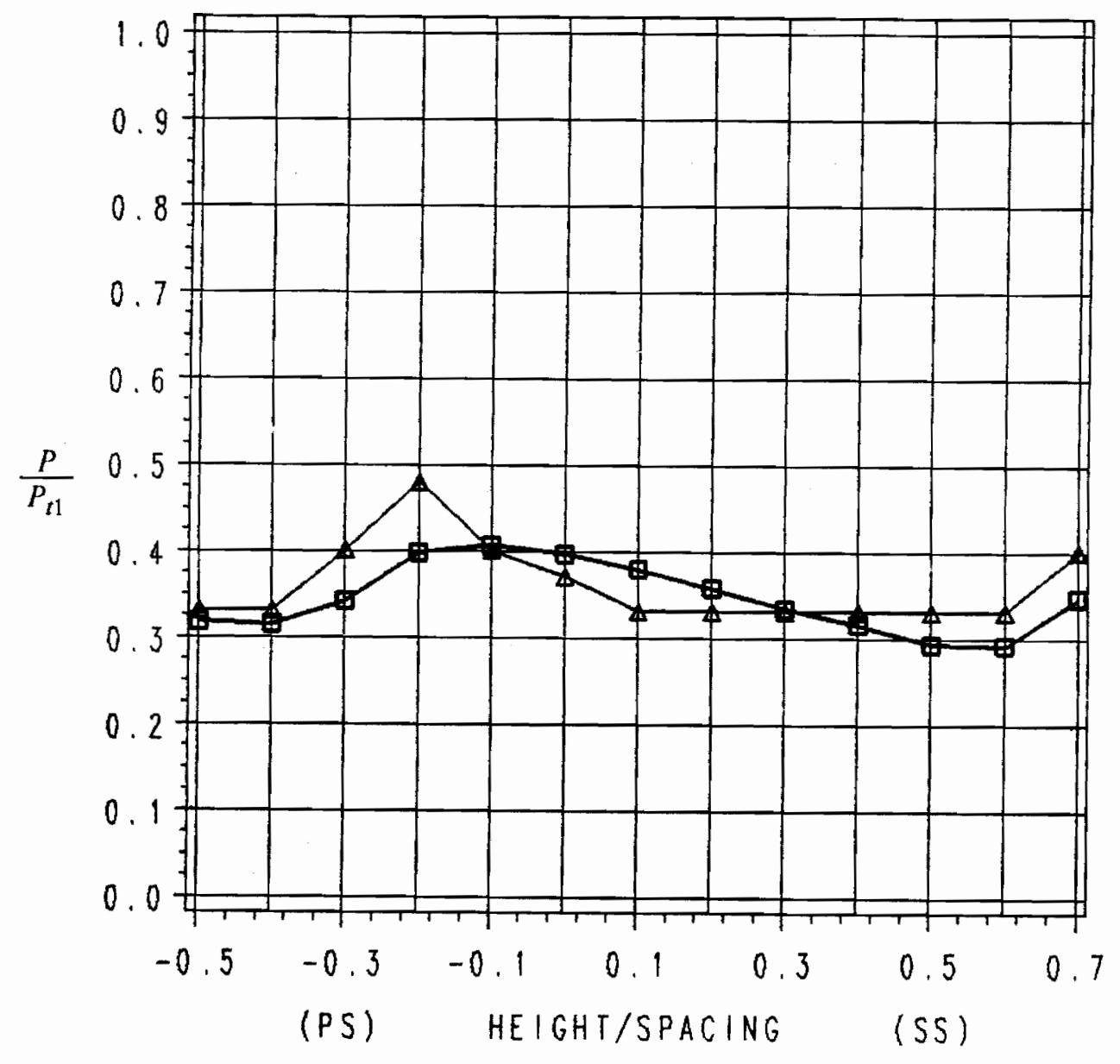

Figure 43. Wall static pressure, Baseline cascade, Station 2:

Squares: Experiment, $M_{2 i s}=1.30$

Triangler: Computed, $M_{2 i s}=1.30$ 


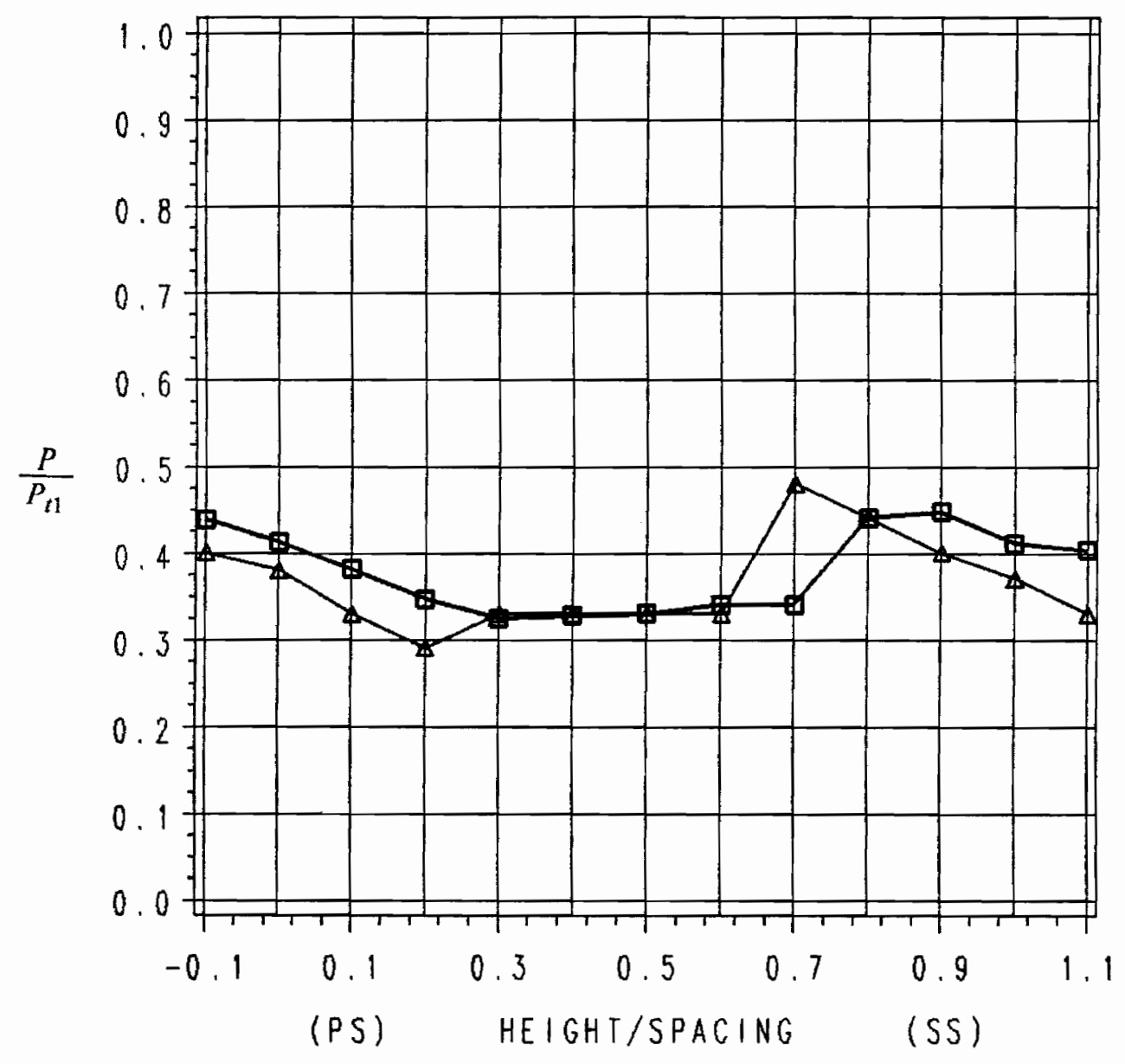

Figure 44. Wall static pressure, OV10 cascade, Station 1:

Squares: Experiment, $M_{2 i s}=1.28$

Triangles: Computed, $M_{2 i s}=1.29$ 


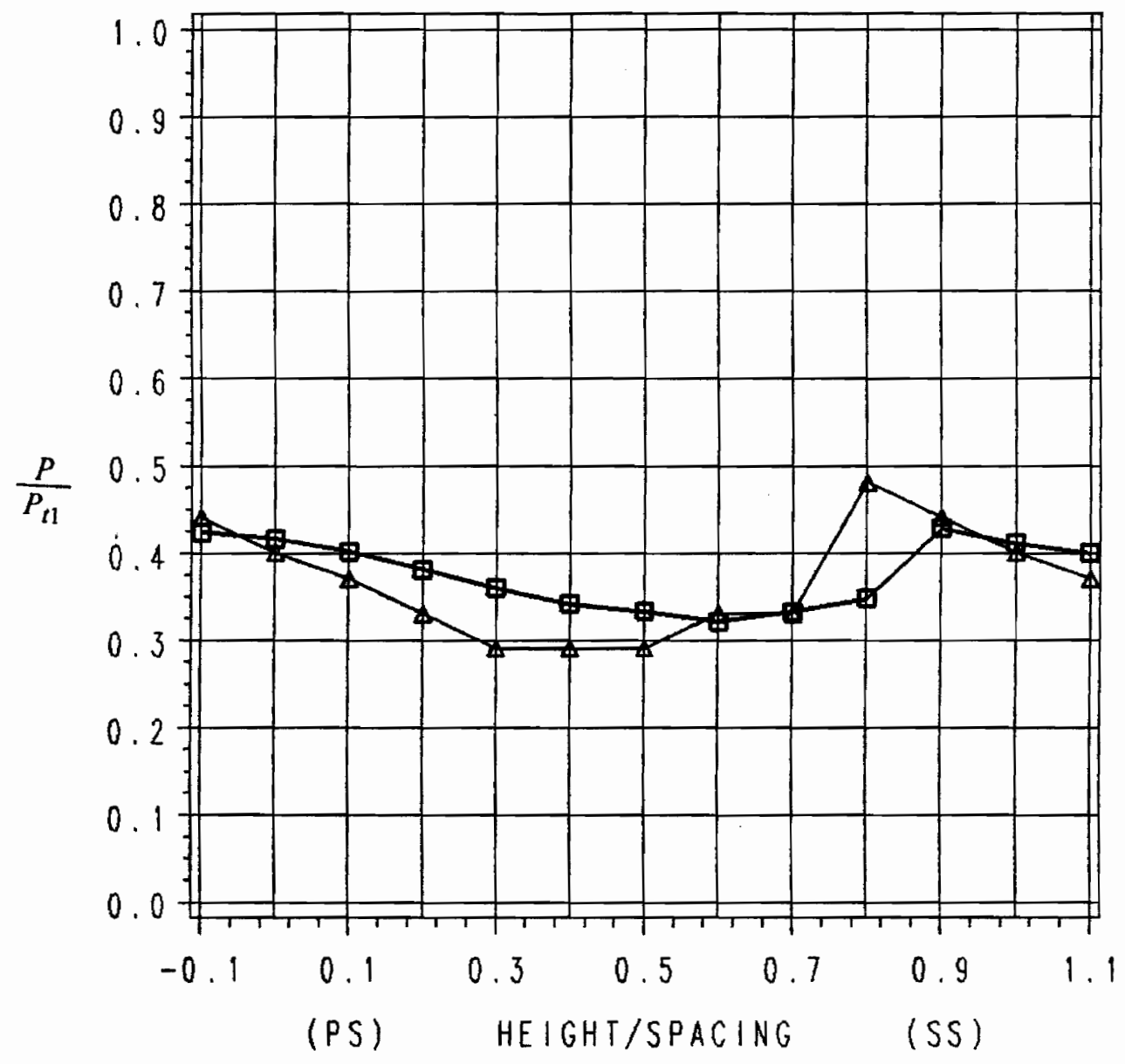

Figure 45. Wall static pressure, OV10 cascade, Station 2:

Squares: Experiment, $M_{2 i s}=1.28$

Triangles: Computed, $M_{2 i s}=1.29$ 


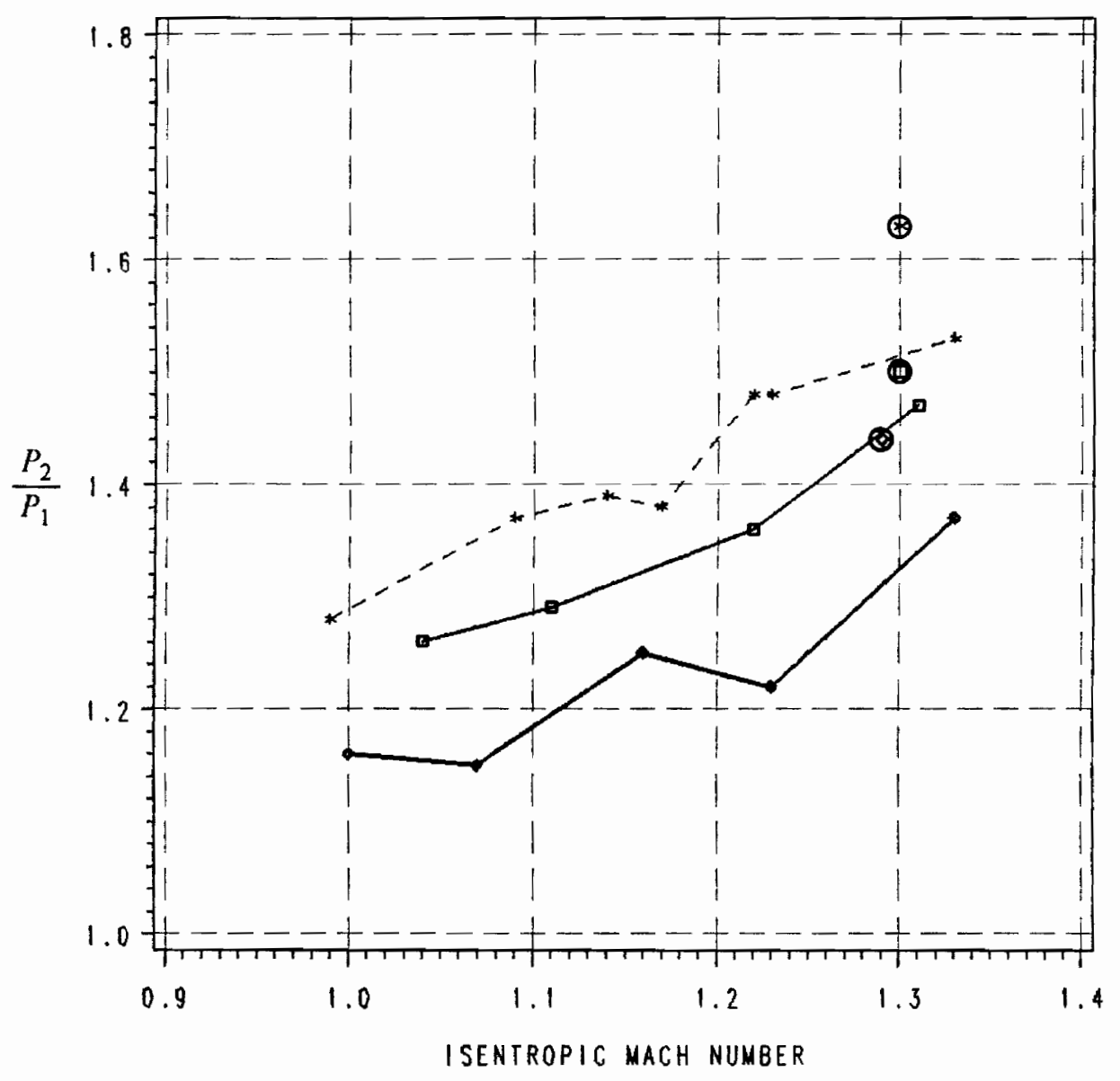

Figure 46. Static pressure rise across suction side shock, Station 1:

$P_{2}=$ Static pressure downstream of shock, $P_{1}=$ upstream of shock

Stars: UN10, $-10 \%$ solidity

Squares: Baseline solidity

Diamonds: OV10, $+10 \%$ solidity

Experiment $=$ connected points, Inviscid prediction $=$ circled points 


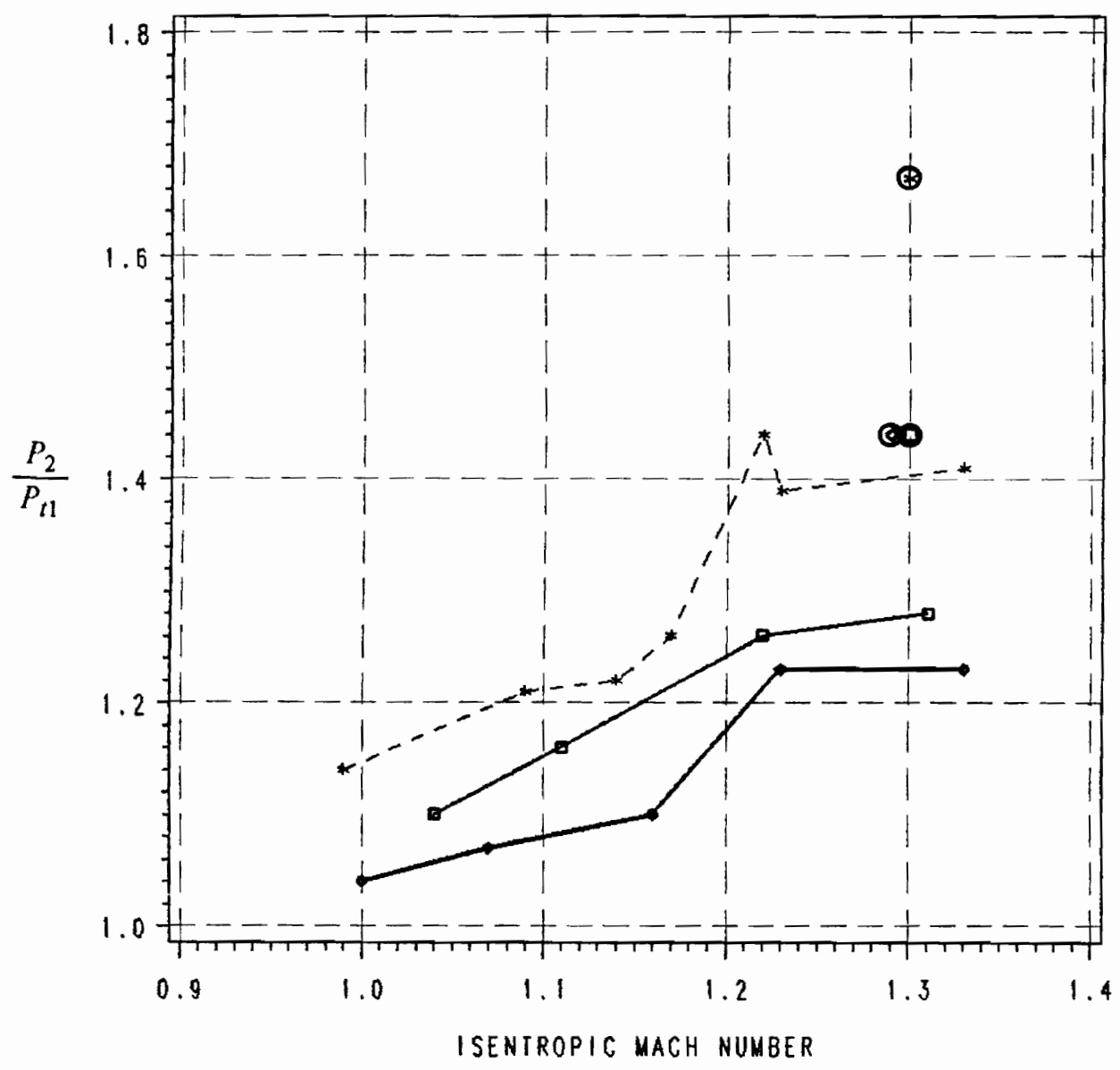

Figure 47. Static pressure rise across suction side shock, Station 2:

$P_{2}=$ Static pressure downstream of shock, $P_{1}=$ upstream of shock

Stars: UN10, $-10 \%$ solidity

Squares: Baseline solidity

Diamonds: OV10, $+10 \%$ solidity

Experiment $=$ connected points, Inviscid prediction $=$ circled points 


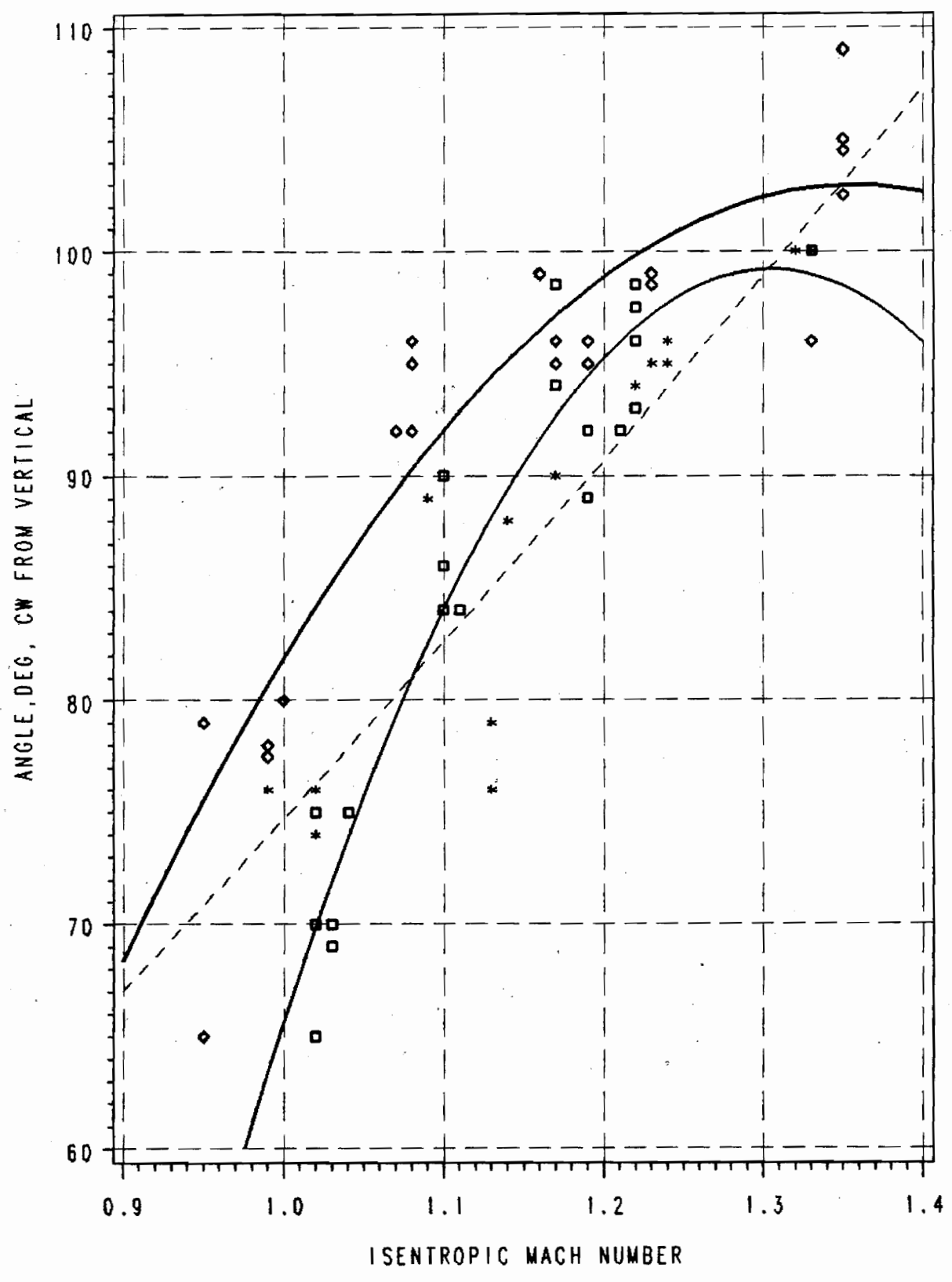

Figure 48. Suction side shock angle versus isentropic Mach number:

Stars $=$ UN10, Squares $=$ Baseline, Diamonds $=$ OV10.

Shock angle measured clockwise from vertical at blade trailing edge. 


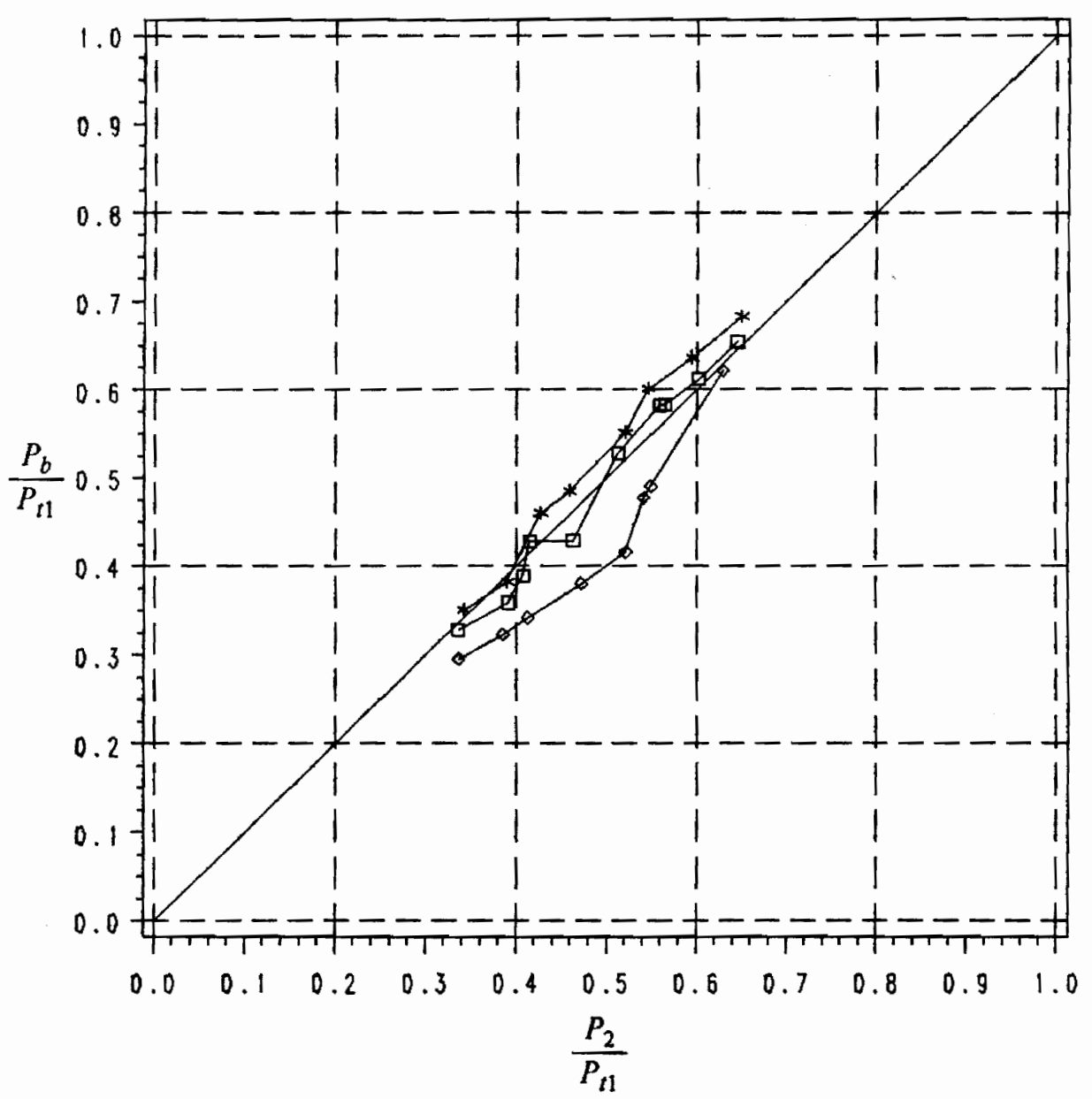

Figure 49. Blade base pressure ratio versus downstream pressure ratio: Stars $=$ UN10, Squares $=$ Baseline, Diamonds $=$ OV10. 


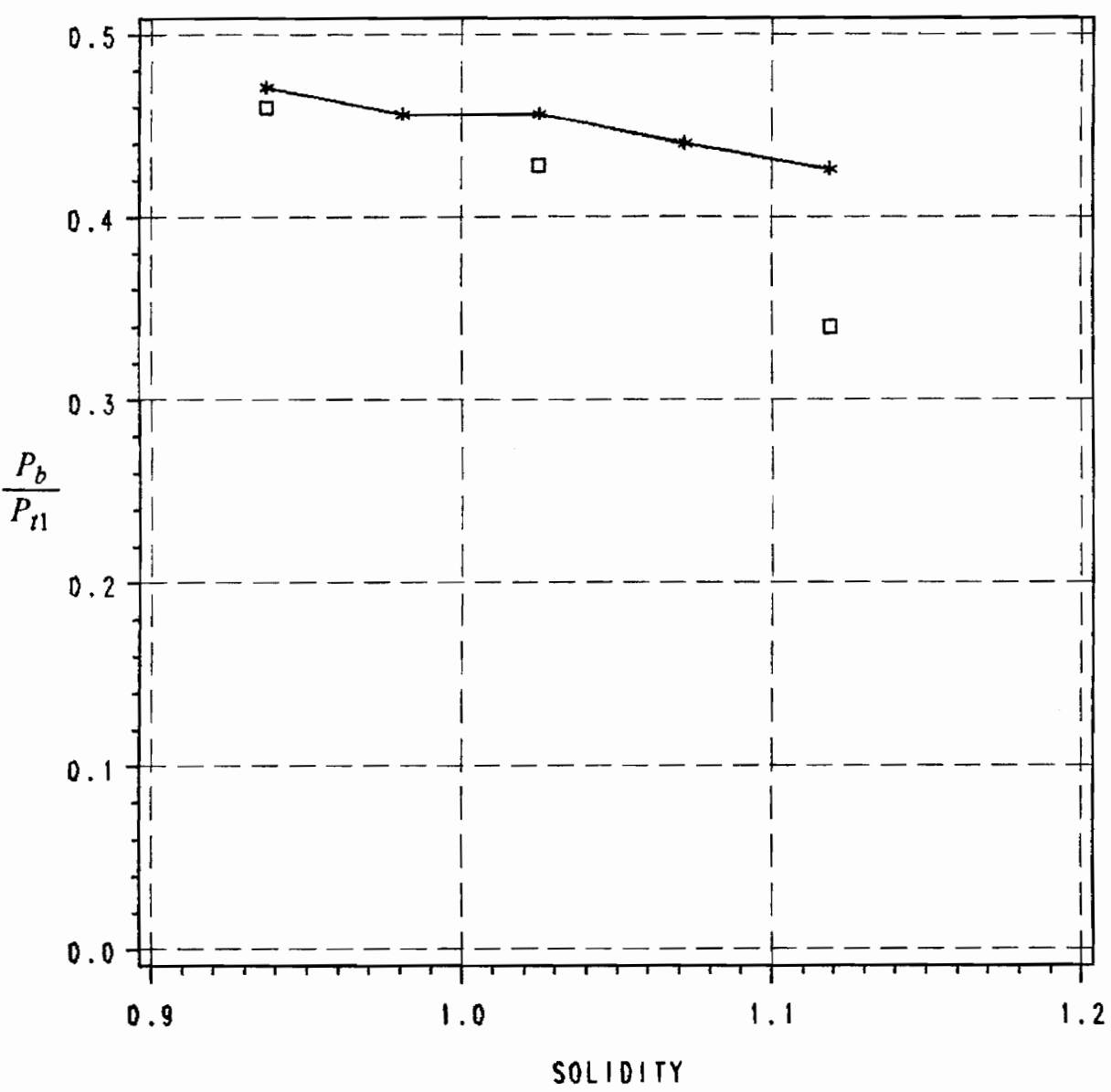

Figure 50. Comparison of experimental and predicted blade base pressures, $\mathbf{M}_{\mathbf{2}}=\mathbf{1 . 3 0}$ : Squares $=$ experimental, Stars $=$ predicted. 

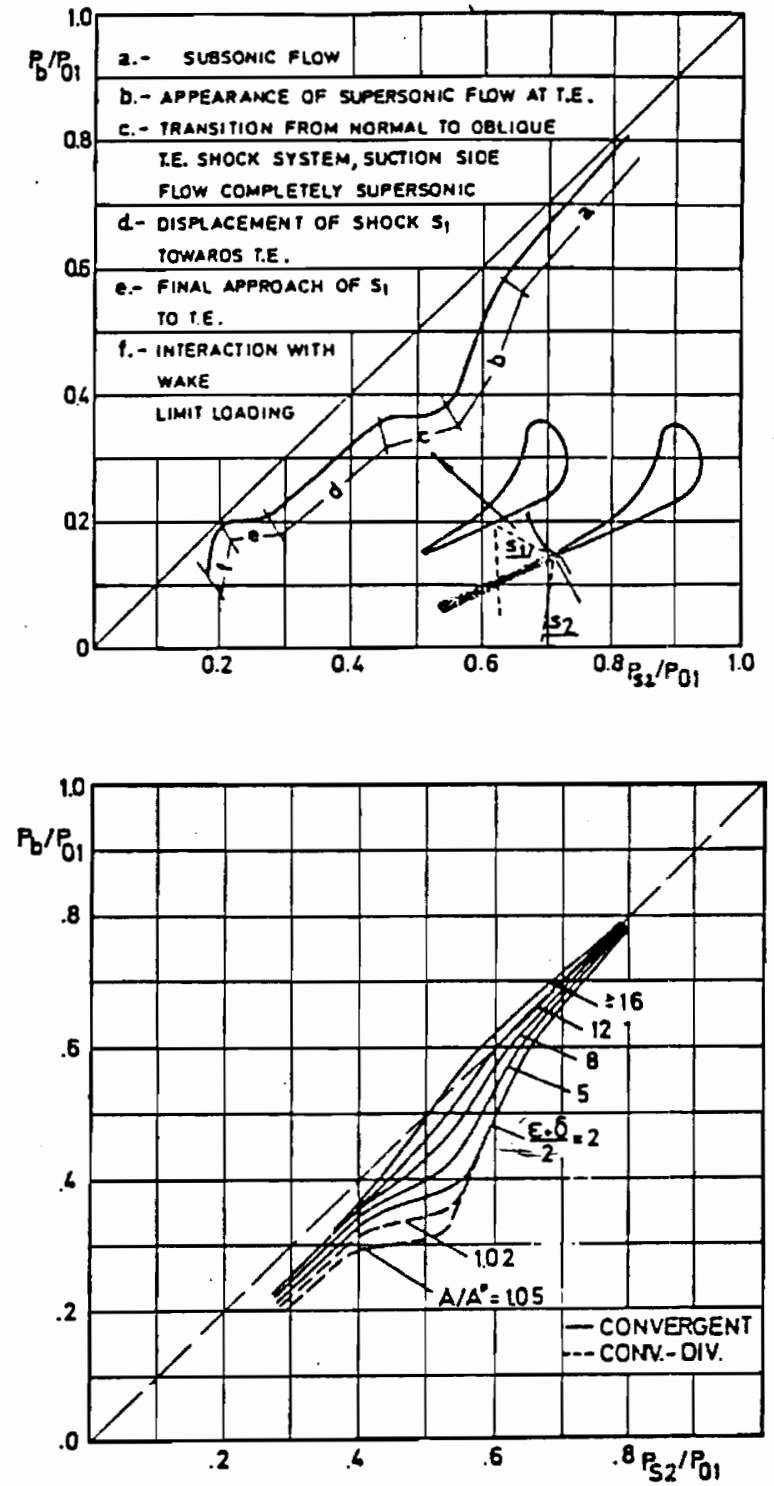

Figure 51. Sieverding's base pressure correlation:

$P_{b}=$ blade base pressure, $\varepsilon=$ unguided turning (UGT), $\delta=$ trailing edge wedge angle. From Reference 32. 


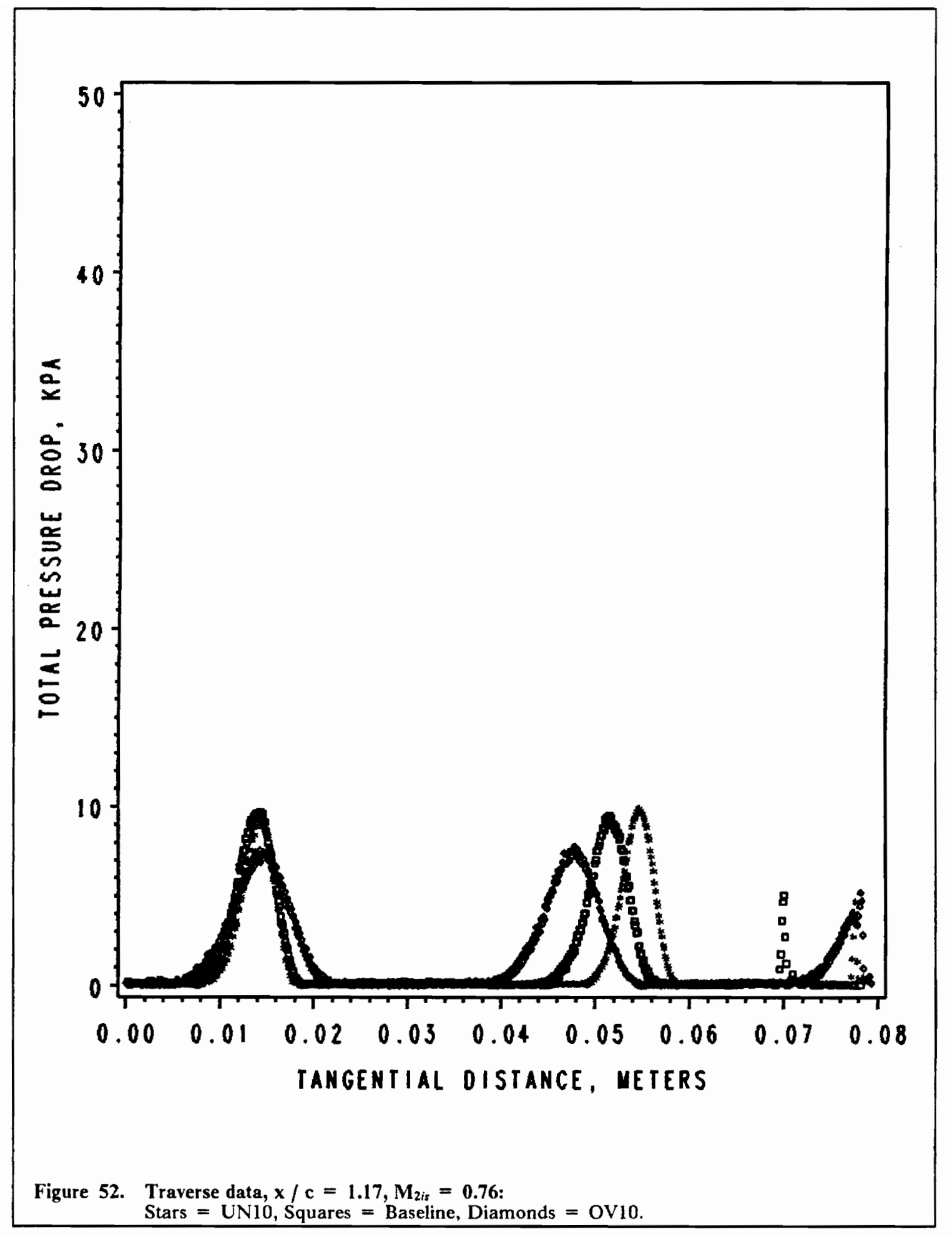




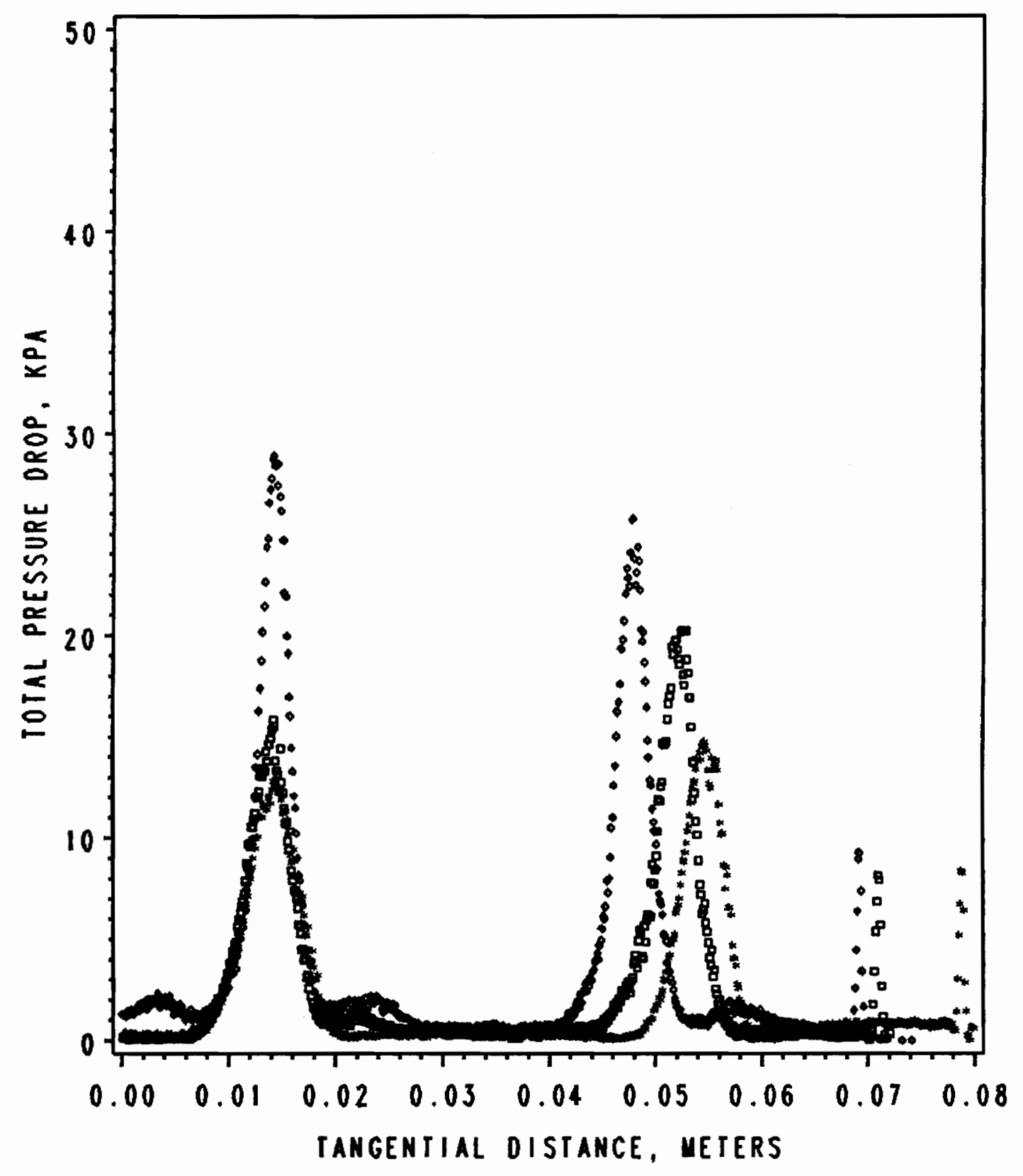

Figure 53. Traverse data, $\mathrm{x} / \mathrm{c}=1.17, \mathrm{M}_{2 i s}=1.00$ :

Stars $=$ UN10, Squares $=$ Baseline, Diamonds $=$ OV10. 


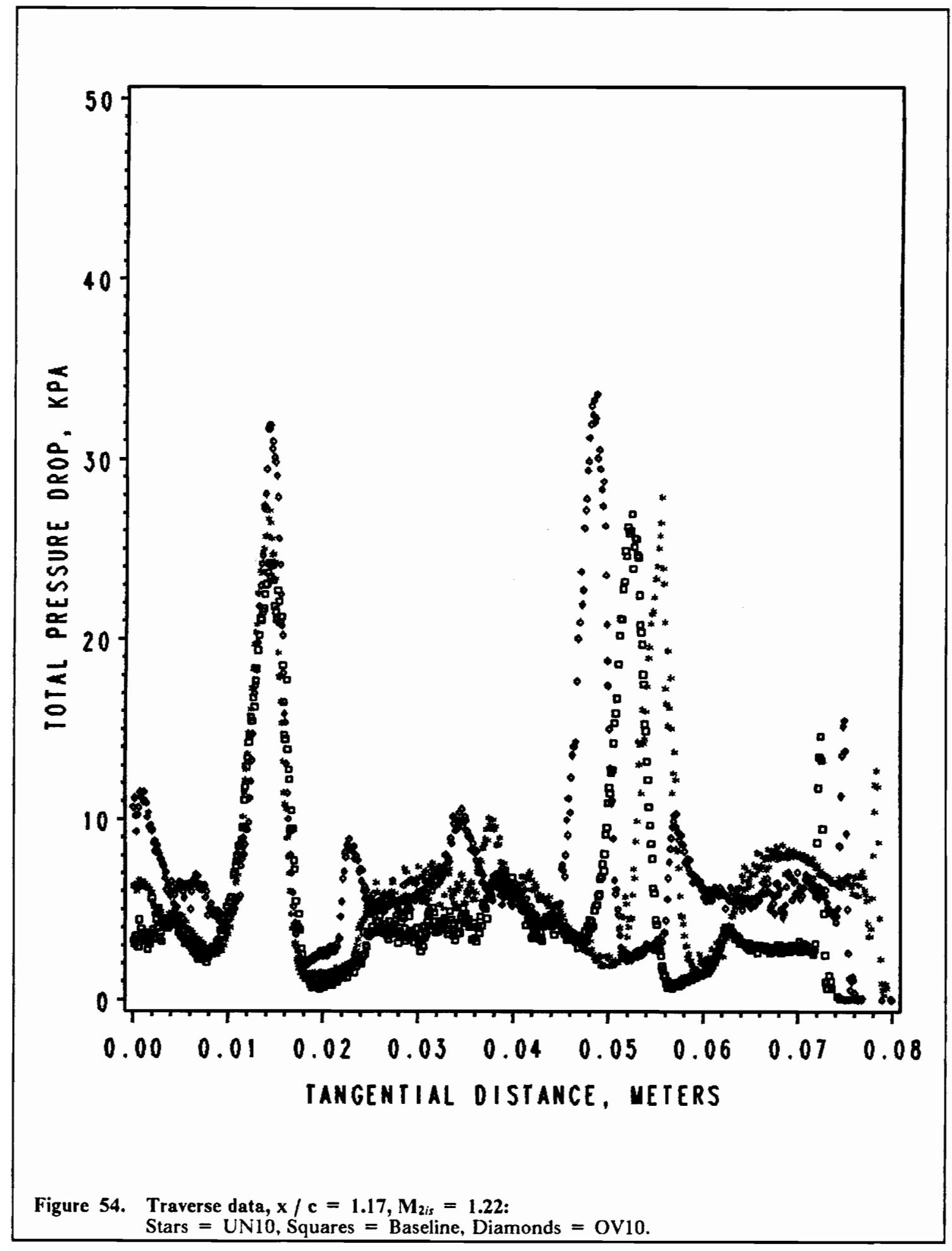




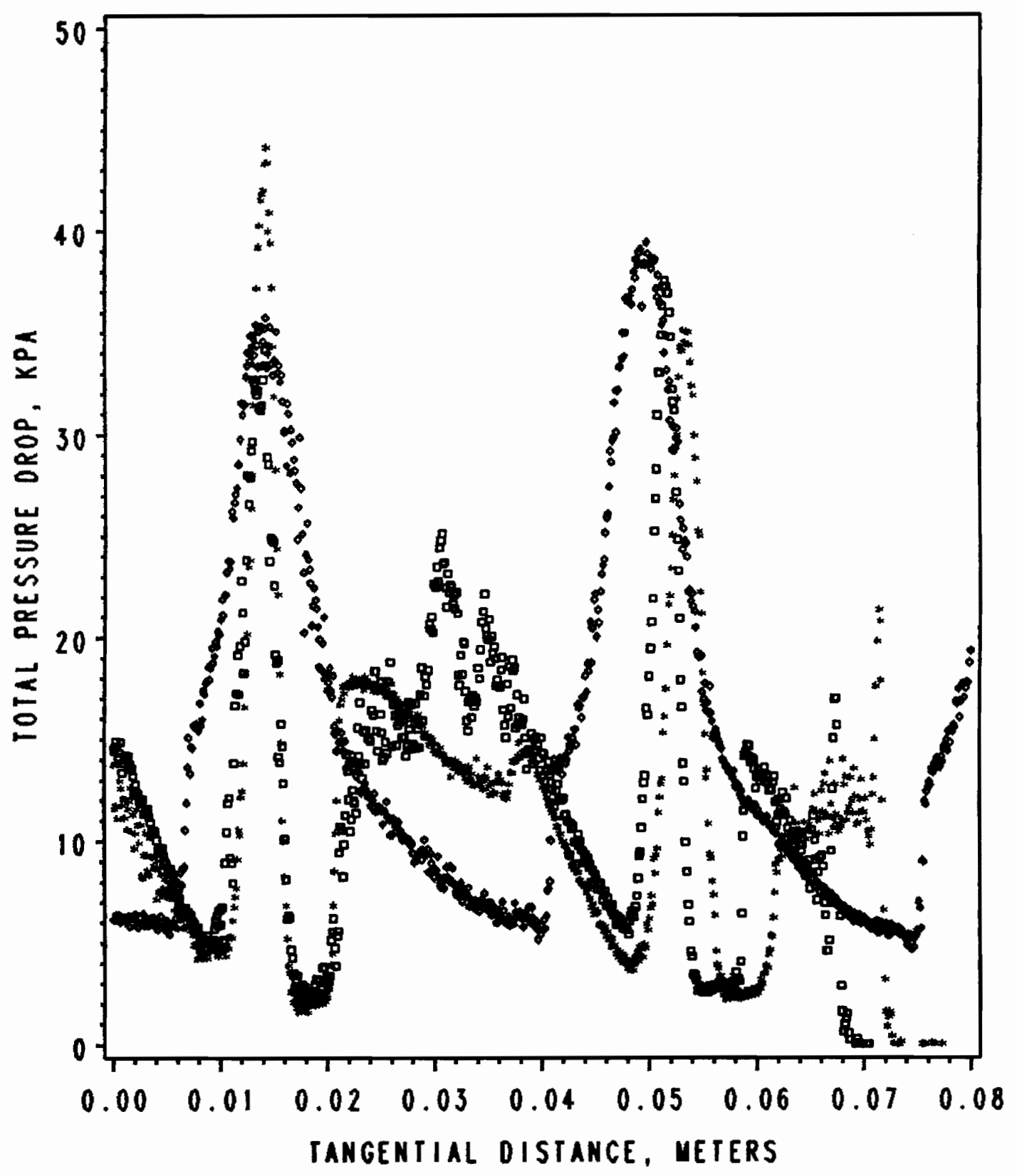

Figure 55. Traverse data, $\mathrm{x} / \mathrm{c}=1.17, \mathrm{M}_{2 \text { is }}=1.35$ :

Stars = UN10, Squares = Baseline, Diamonds $=$ OV10. 


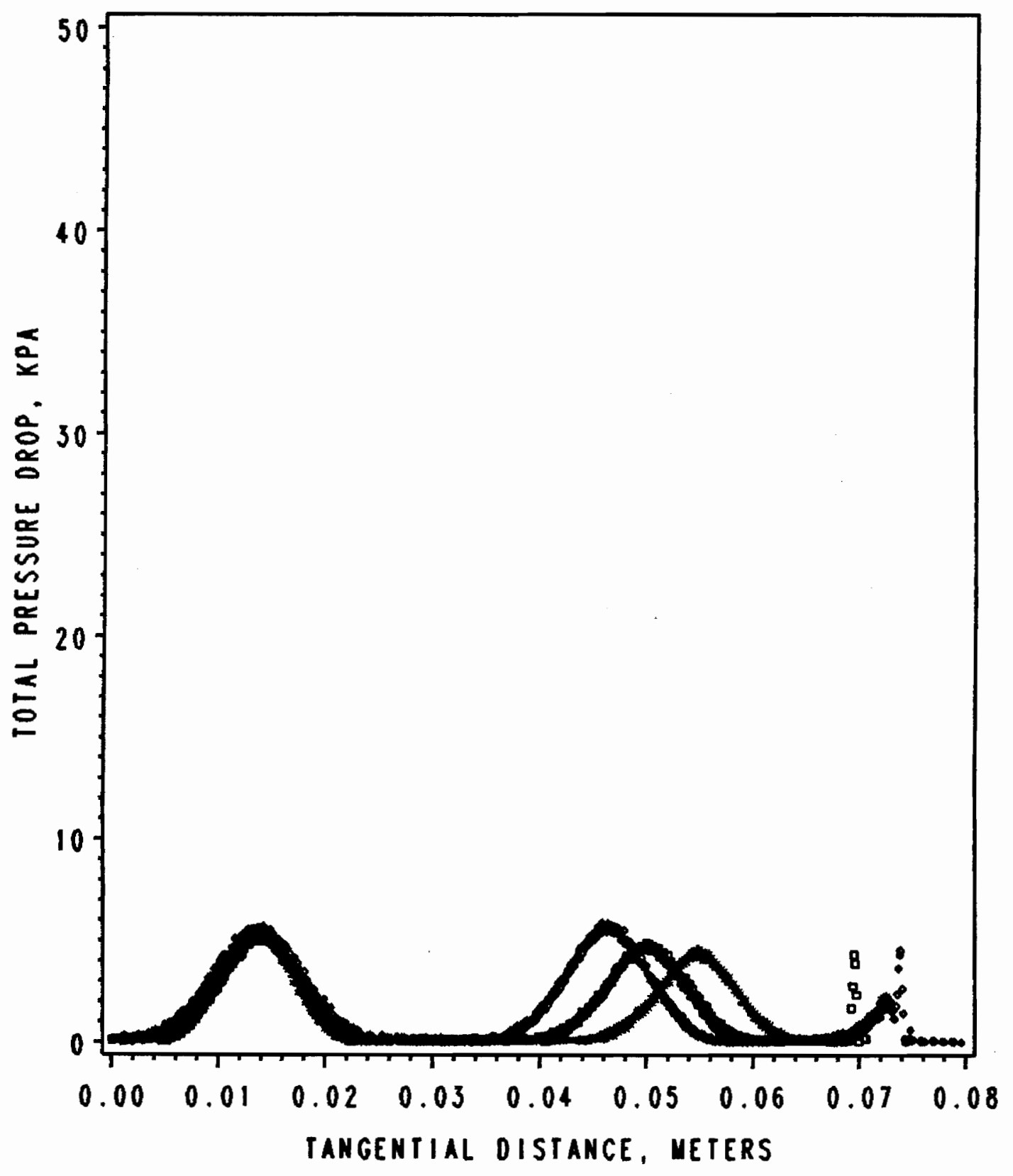

Figure 56. Traverse data, $x / c=1.67, M_{2 i s}=0.76$ :

Stars $=$ UN10, Squares $=$ Baseline, Diamonds $=$ OV10. 


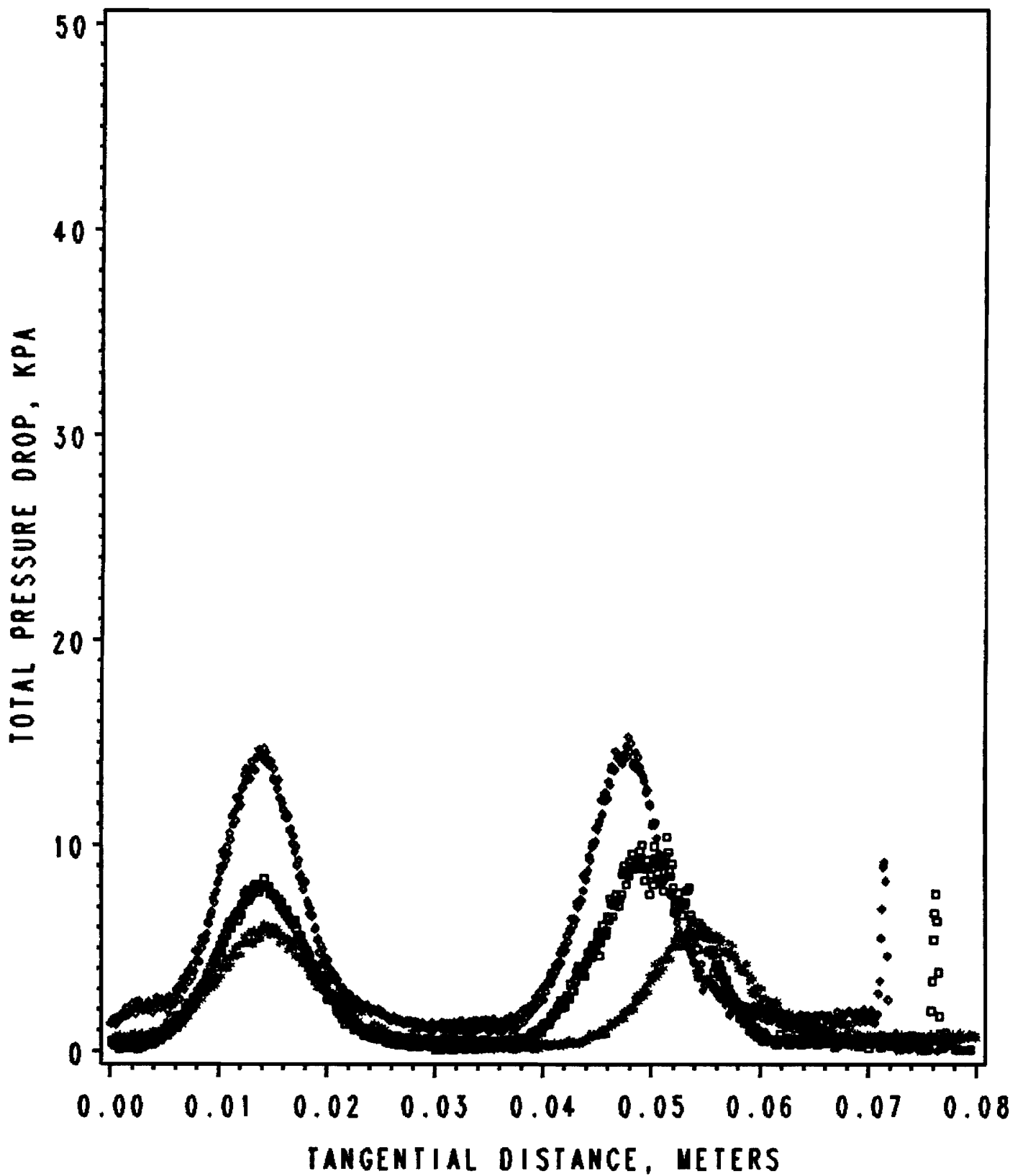

Figure 57. Traverse data, $\mathrm{x} / \mathrm{c}=1.67, \mathrm{M}_{2 \text { is }}=1.00$ :

Stars = UN10, Squares = Baseline, Diamonds = OV10. 


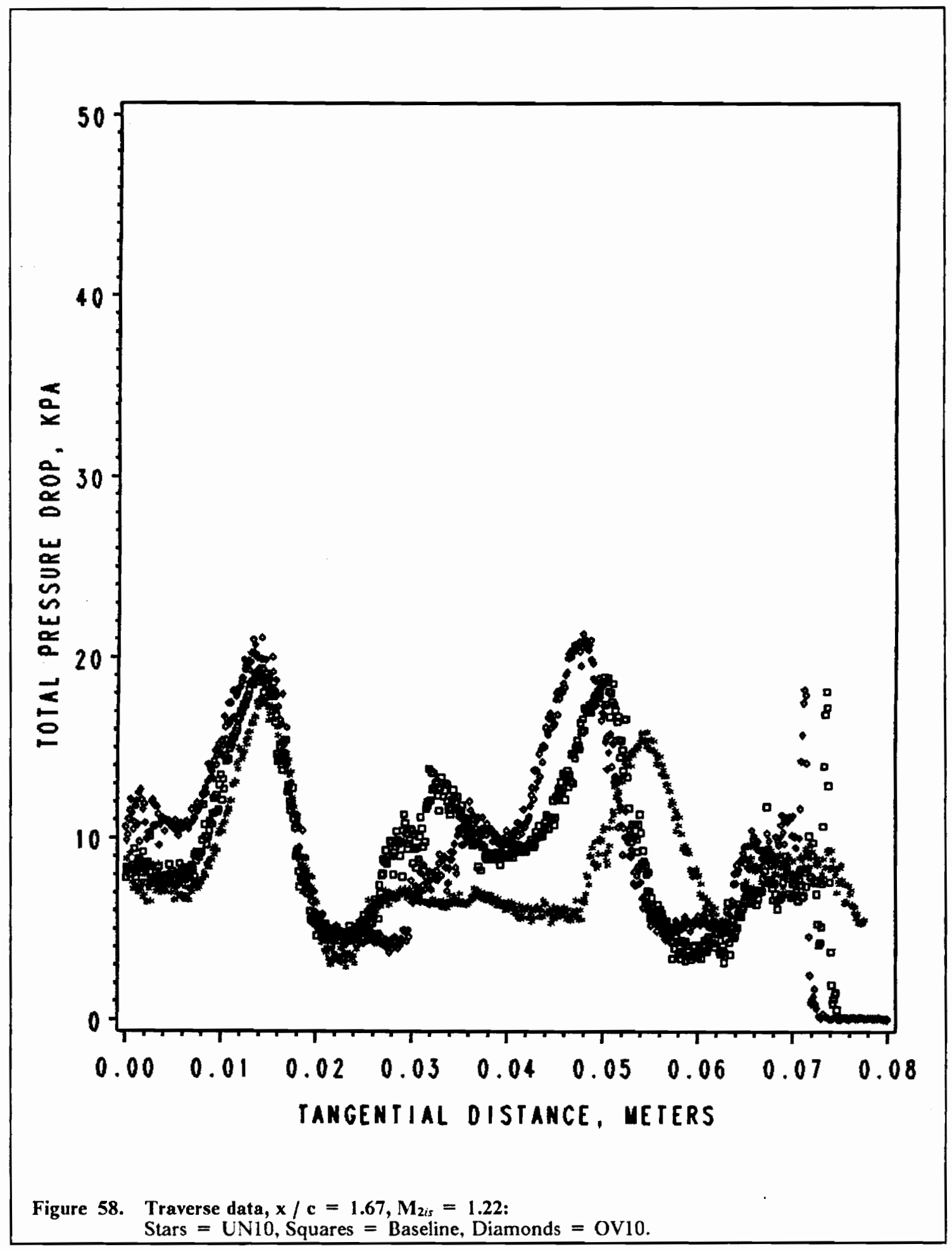




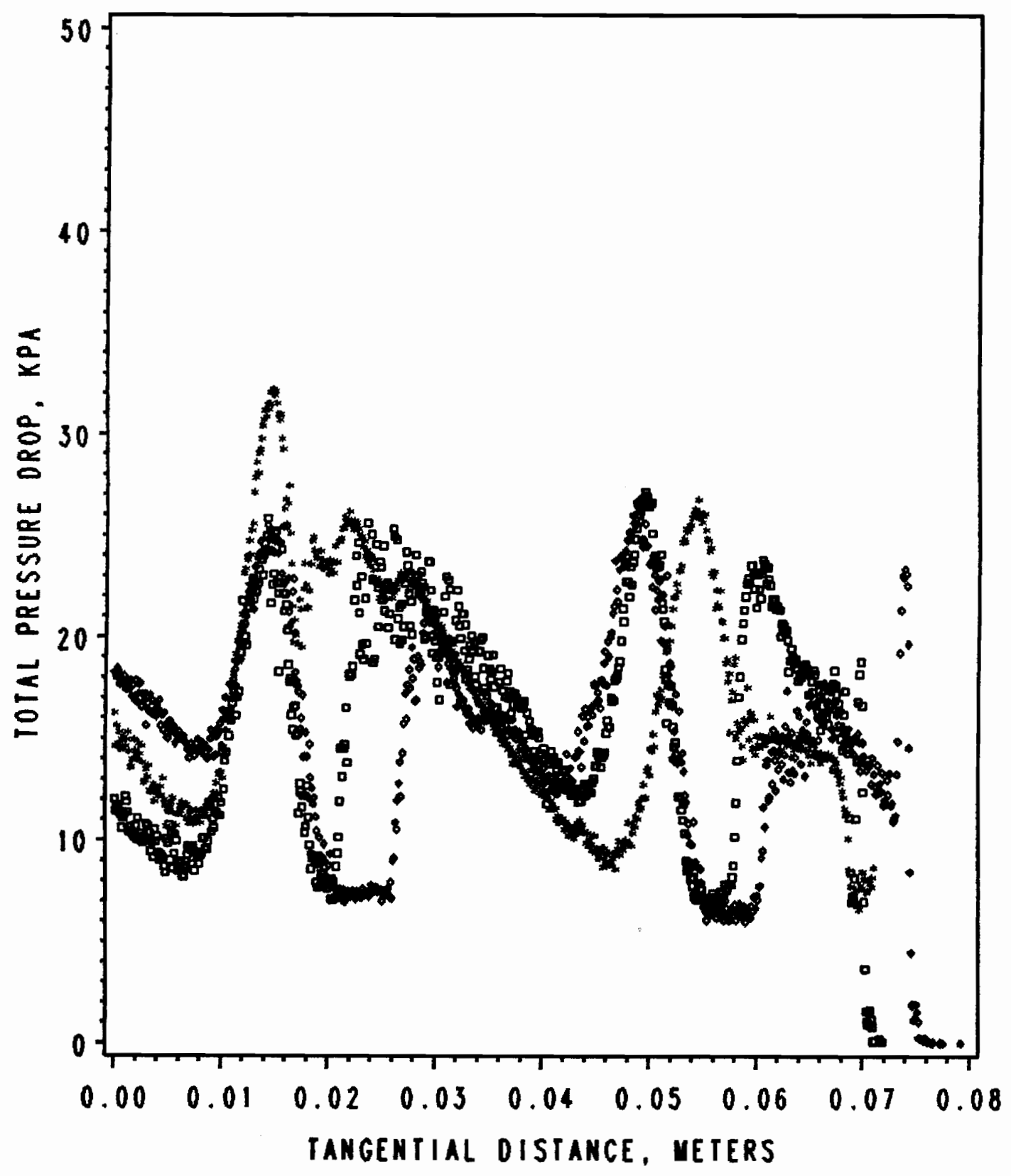

Figure 59. Traverse data, $x / c=1.67, M_{2 i s}=1.35$ :

Stars $=$ UN10, Squares $=$ Baseline, Diamonds $=$ OV10. 


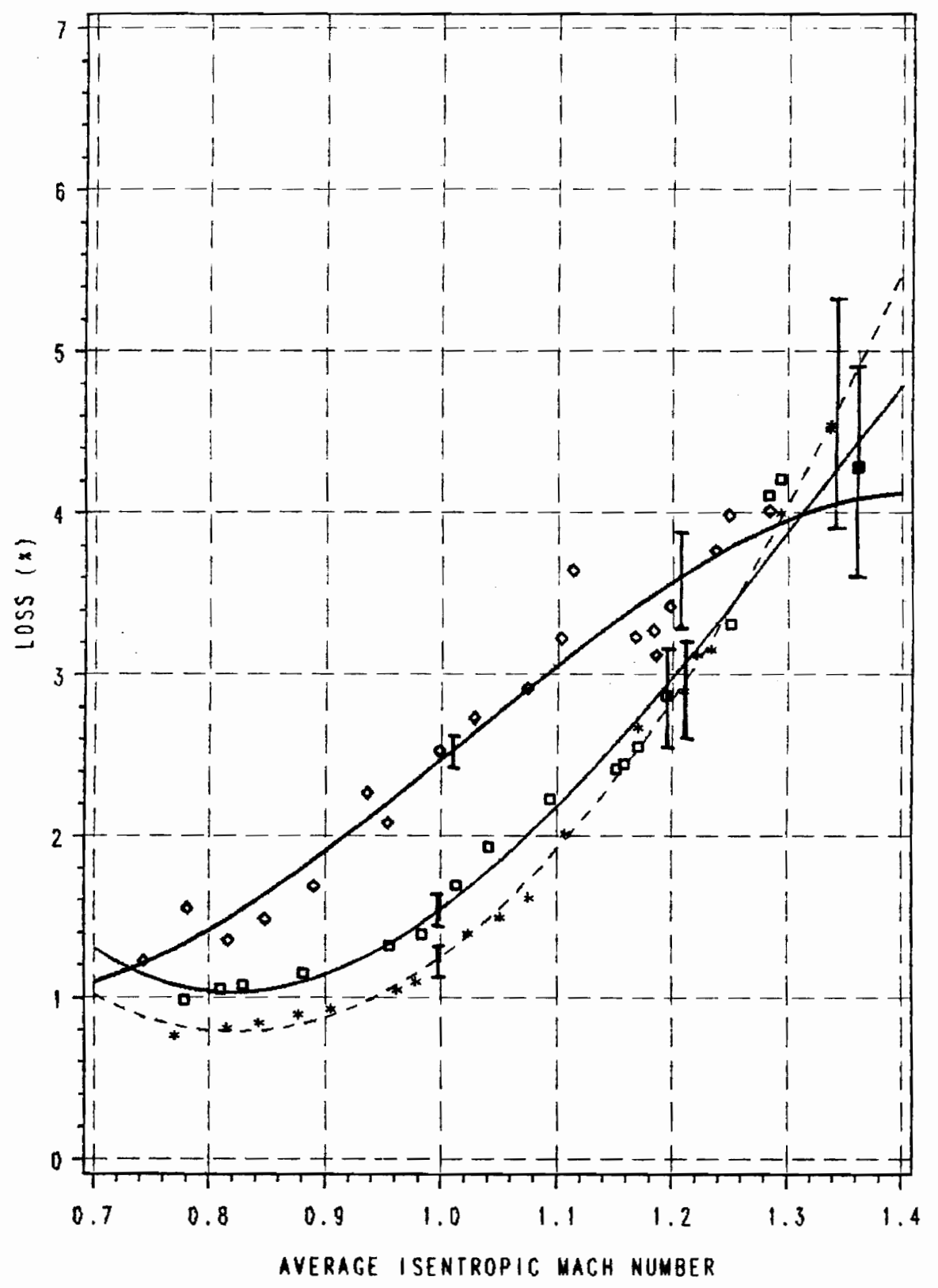

Figure 60. Corrected total pressure loss versus isentropic Mach number, all cascades, Station 1: Stars $=$ UN10, Squares $=$ Baseline, Diamonds $=$ OV10. 


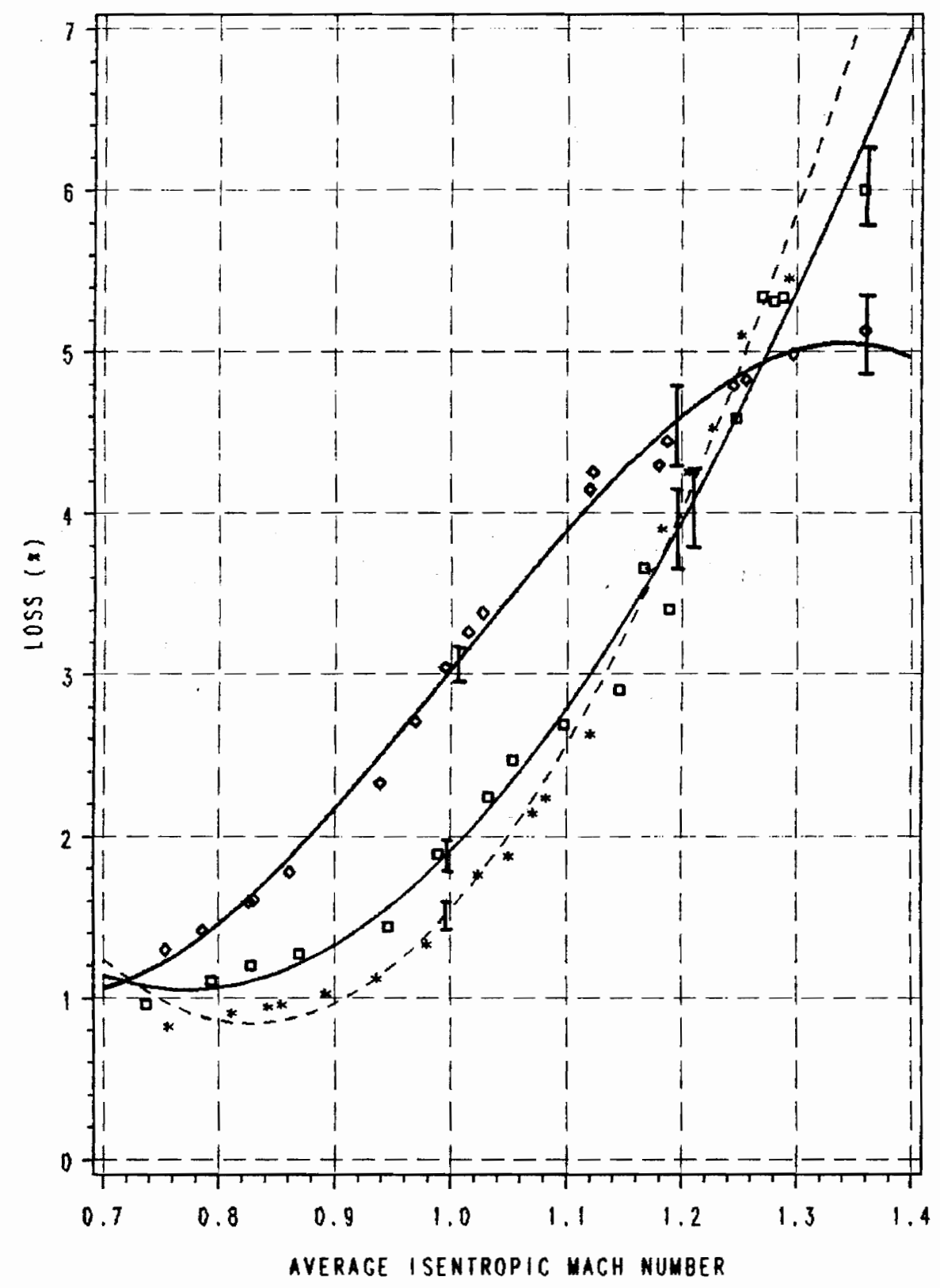

Figure 61. Corrected total pressure loss versus isentropic Mach number, all cascades, Station 2: Stars $=$ UN10, Squares $=$ Baseline, Diamonds $=$ OV10. 


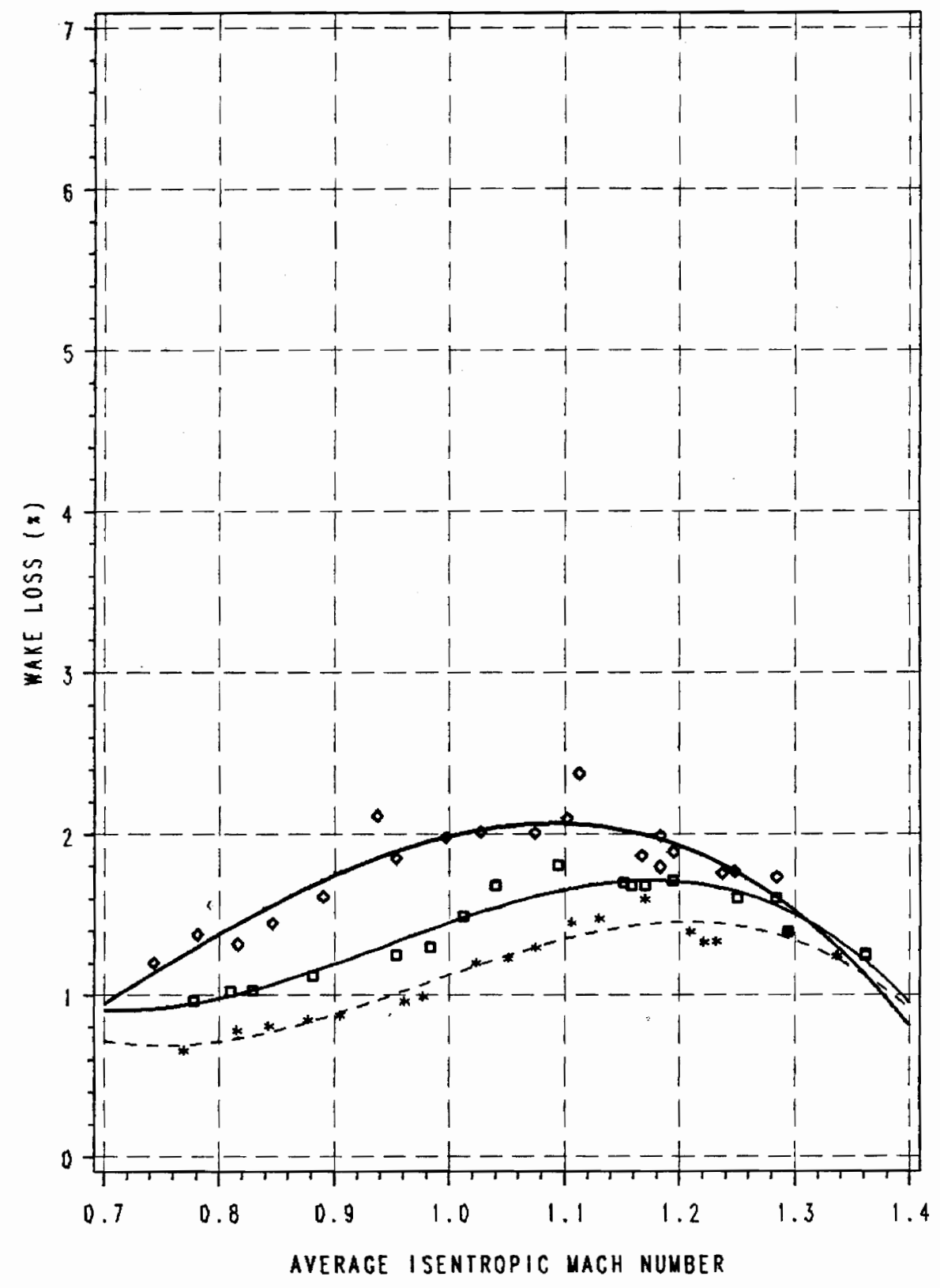

Figure 62. Corrected wake total pressure loss, all cascades, Station 1: Stars $=$ UN10, Squares $=$ Baseline, Diamonds $=$ OV10. 


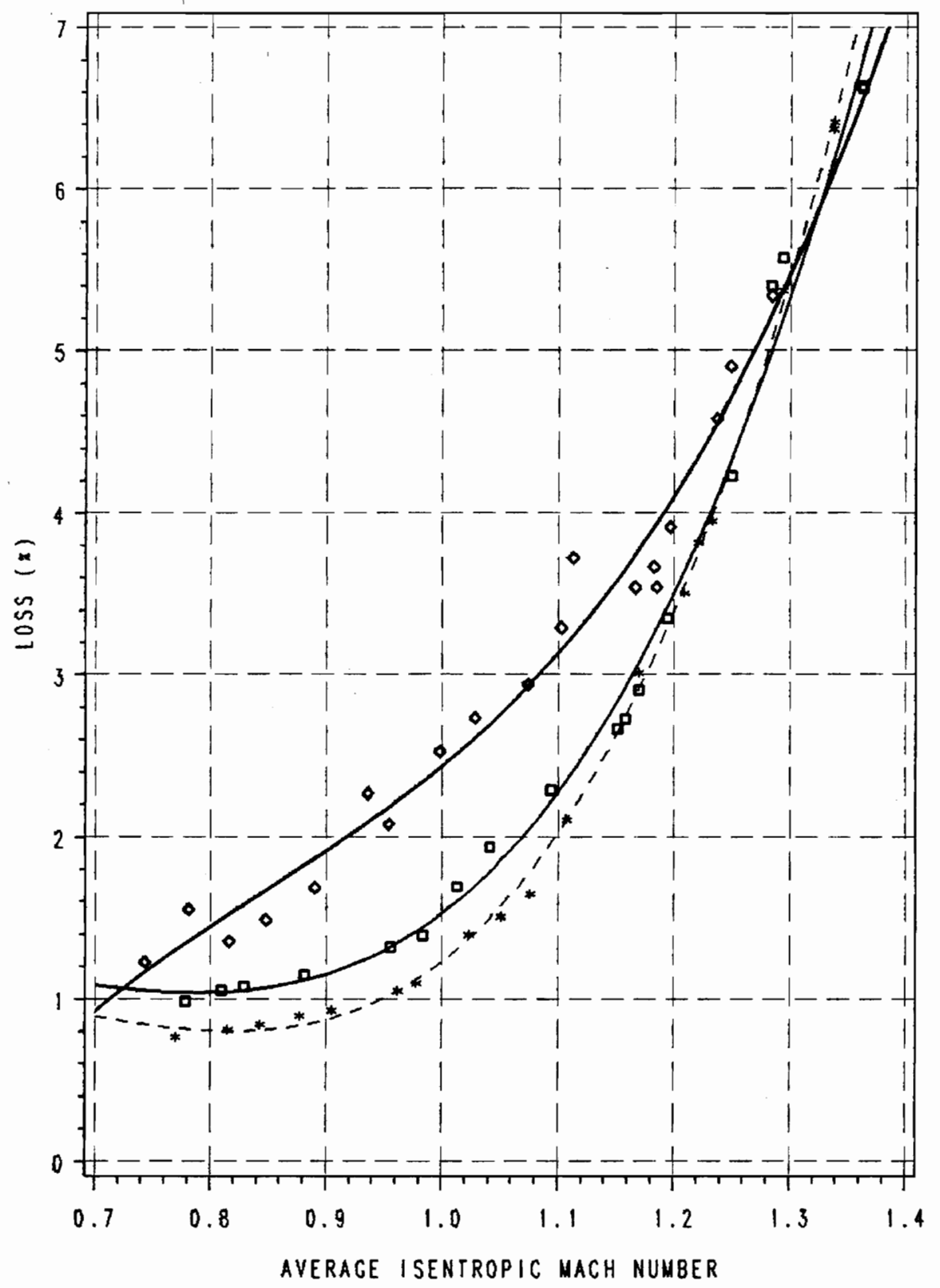

Figure 63. Uncorrected total pressure loss versus isentropic Mach number, all cascades, Station 1: Stars $=\mathrm{UN10}$, Squares $=$ Baseline, Diamonds $=$ OV10. 


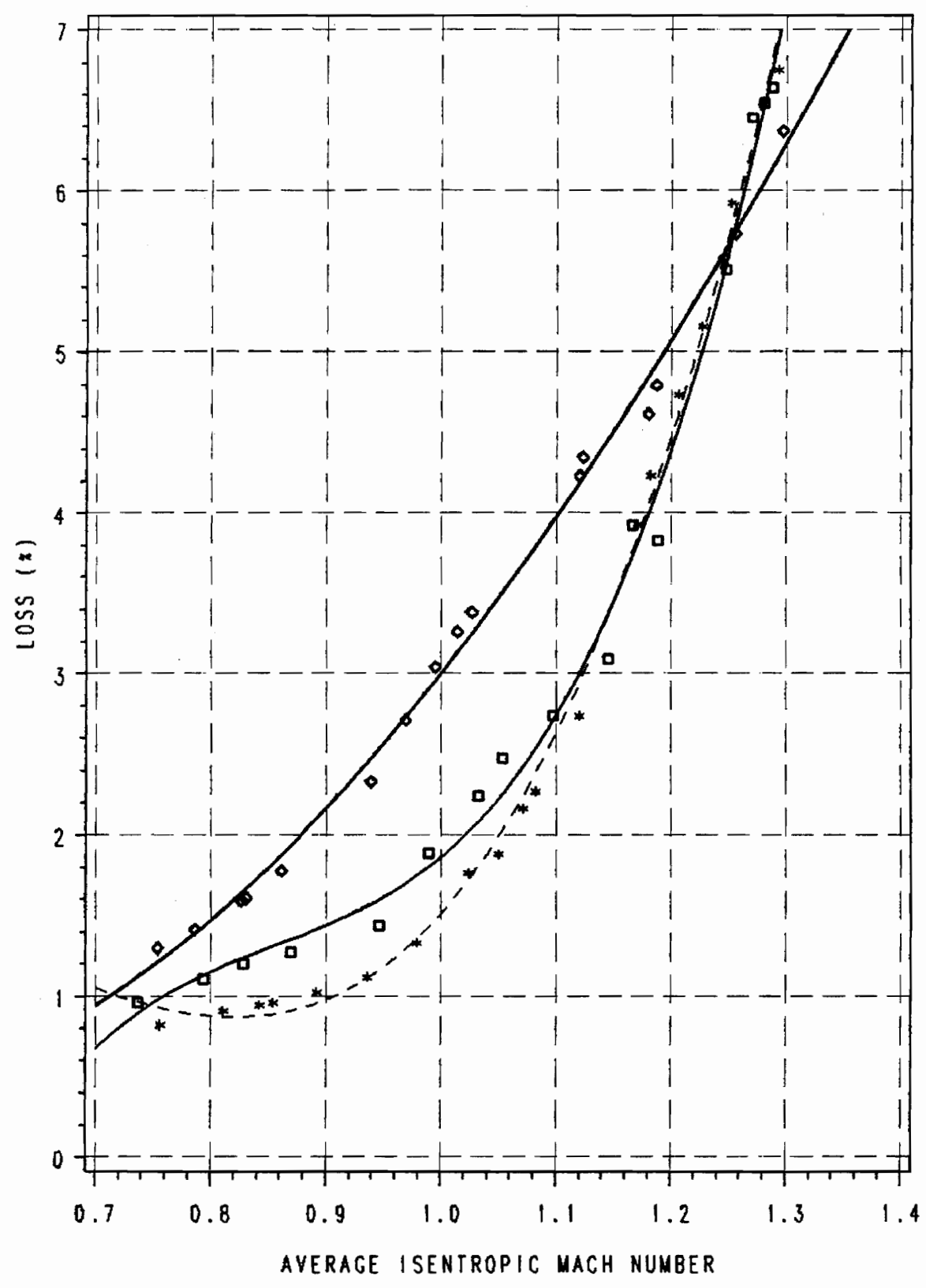

Figure 64. Uncorrected total pressure loss versus isentropic Mach number, all cascades, Station 2: Stars $=$ UN10, Squares $=$ Baseline, Diamonds $=$ OV10. 


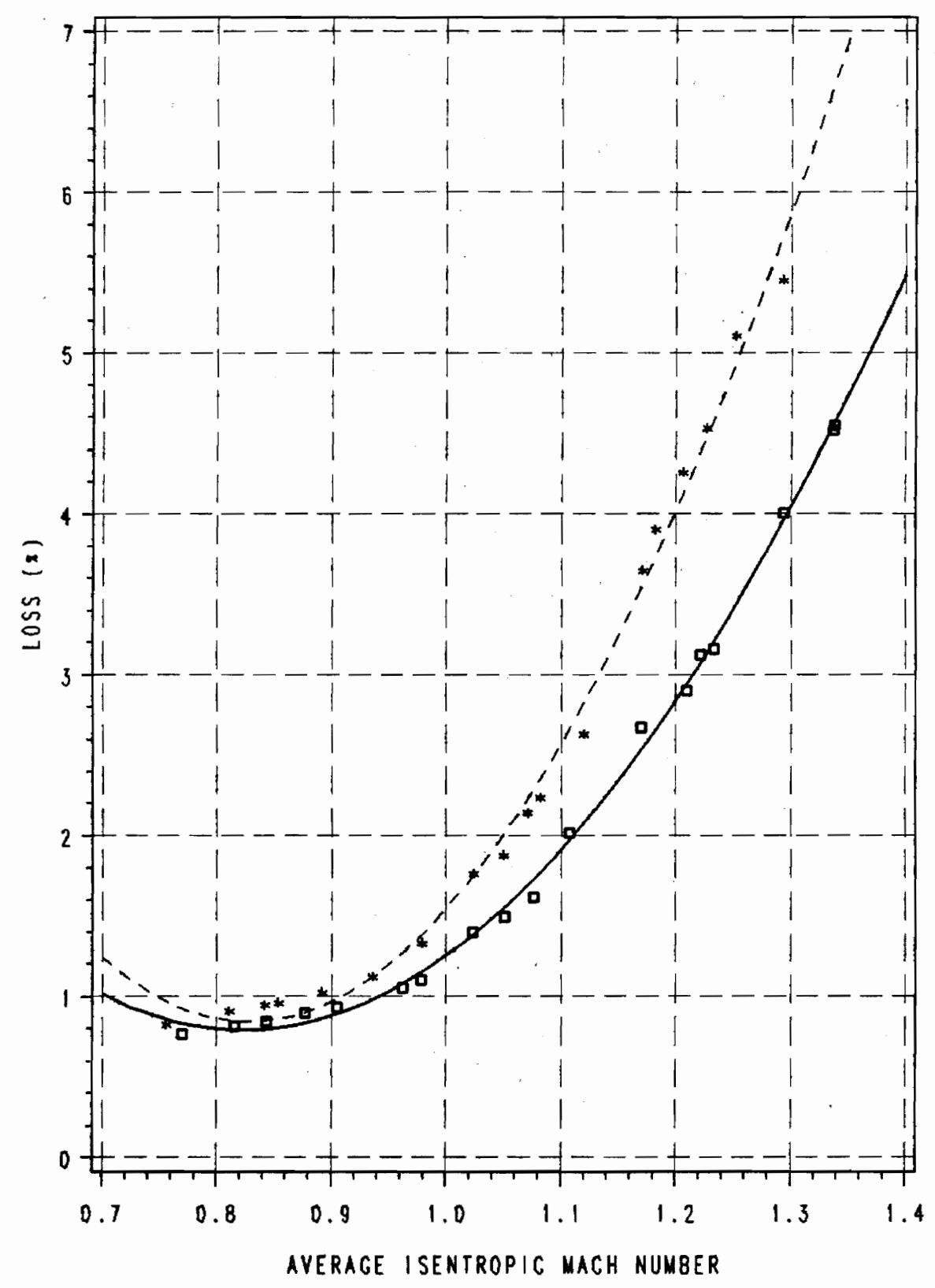

Figure 65. Corrected total pressure loss versus isentropic Mach number, UN10 cascade: Stars $=$ Station 1, Squares $=$ Station 2. 


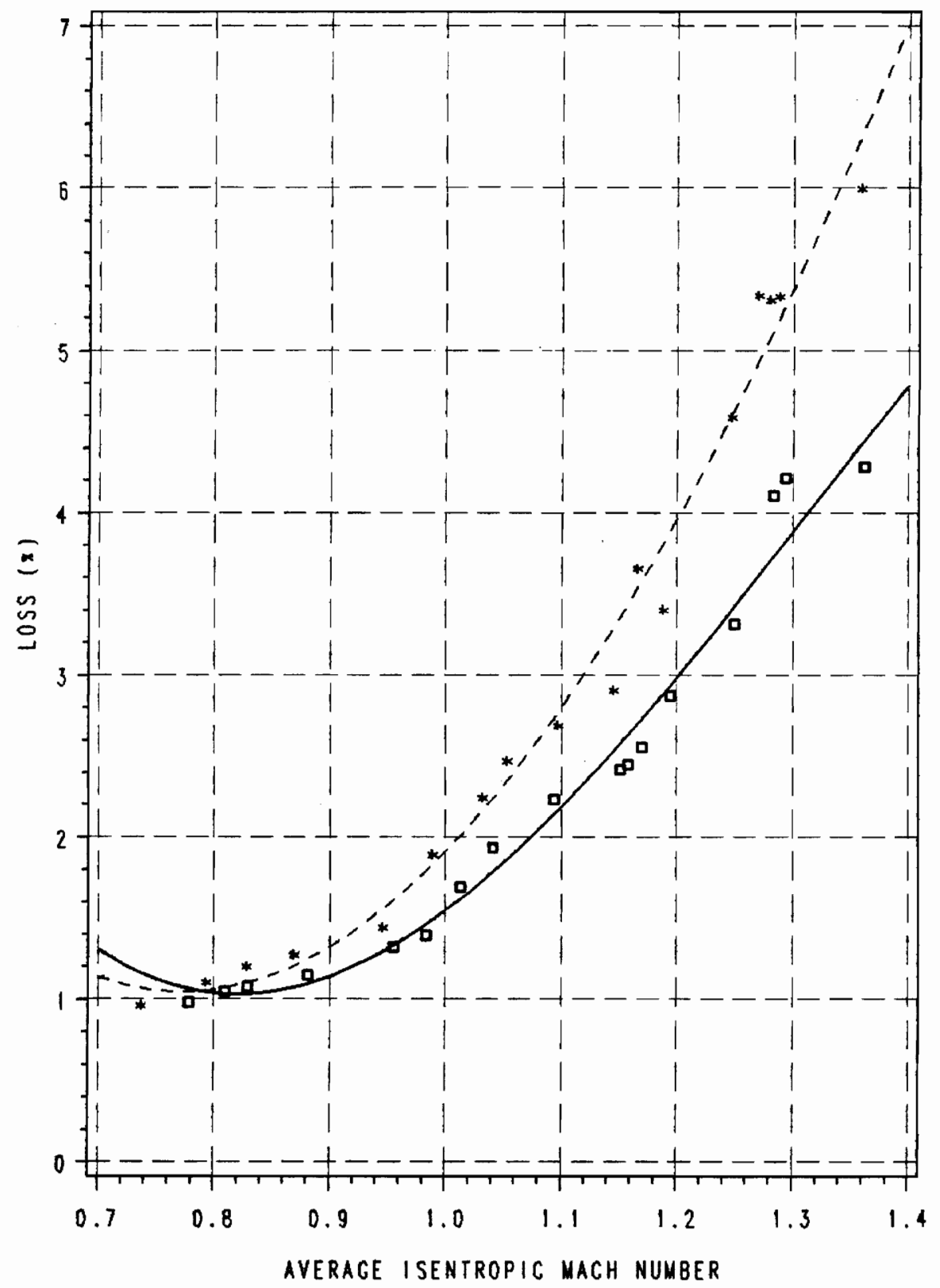

Figure 66. Corrected total pressure loss versus isentropic Mach number, Baseline cascade: Stars = Station 1, Squares = Station 2 . 


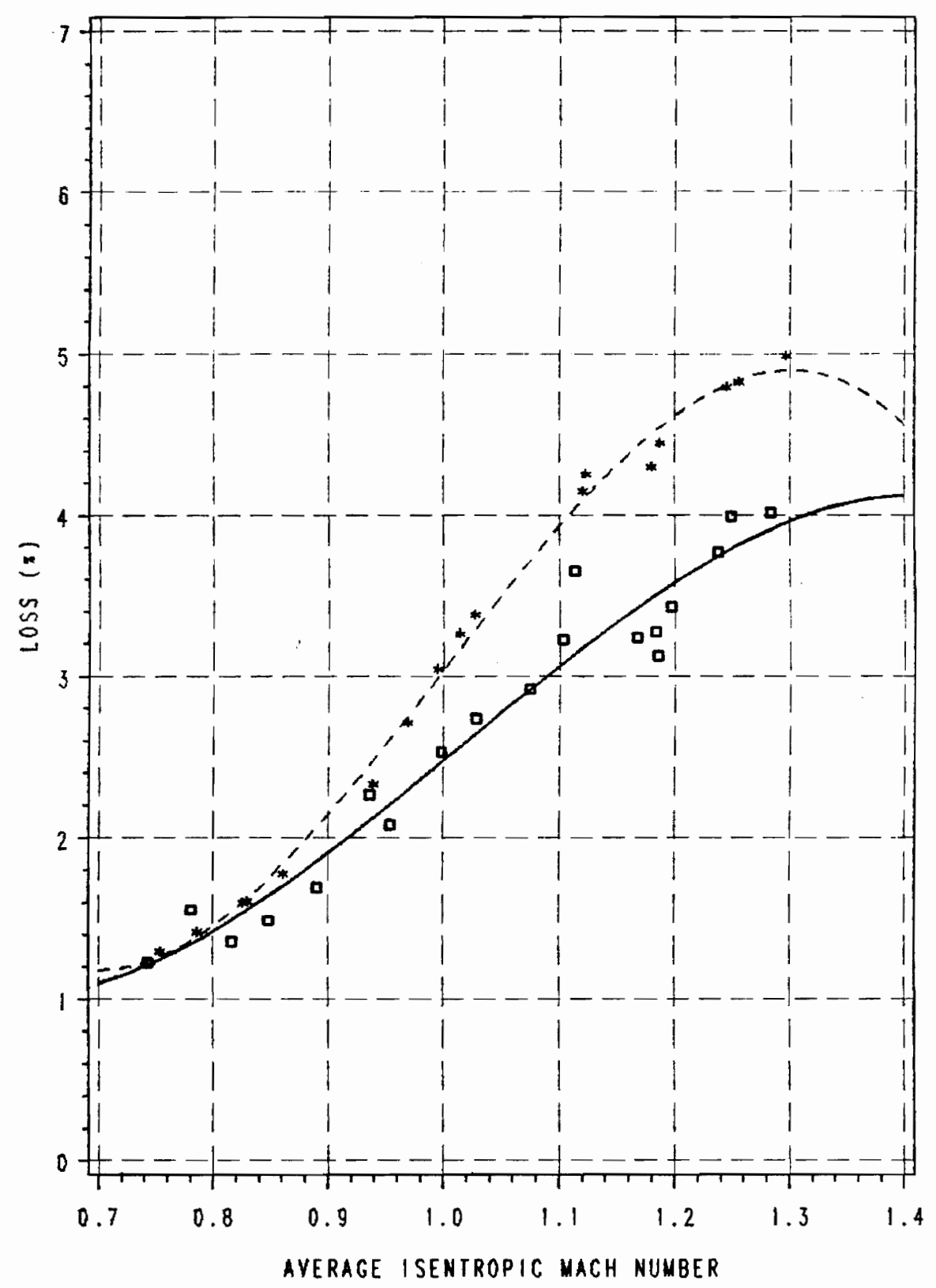

Figure 67. Corrected total pressure loss versus isentropic Mach number, OV10 cascade: Stars = Station 1, Squares = Station 2. 


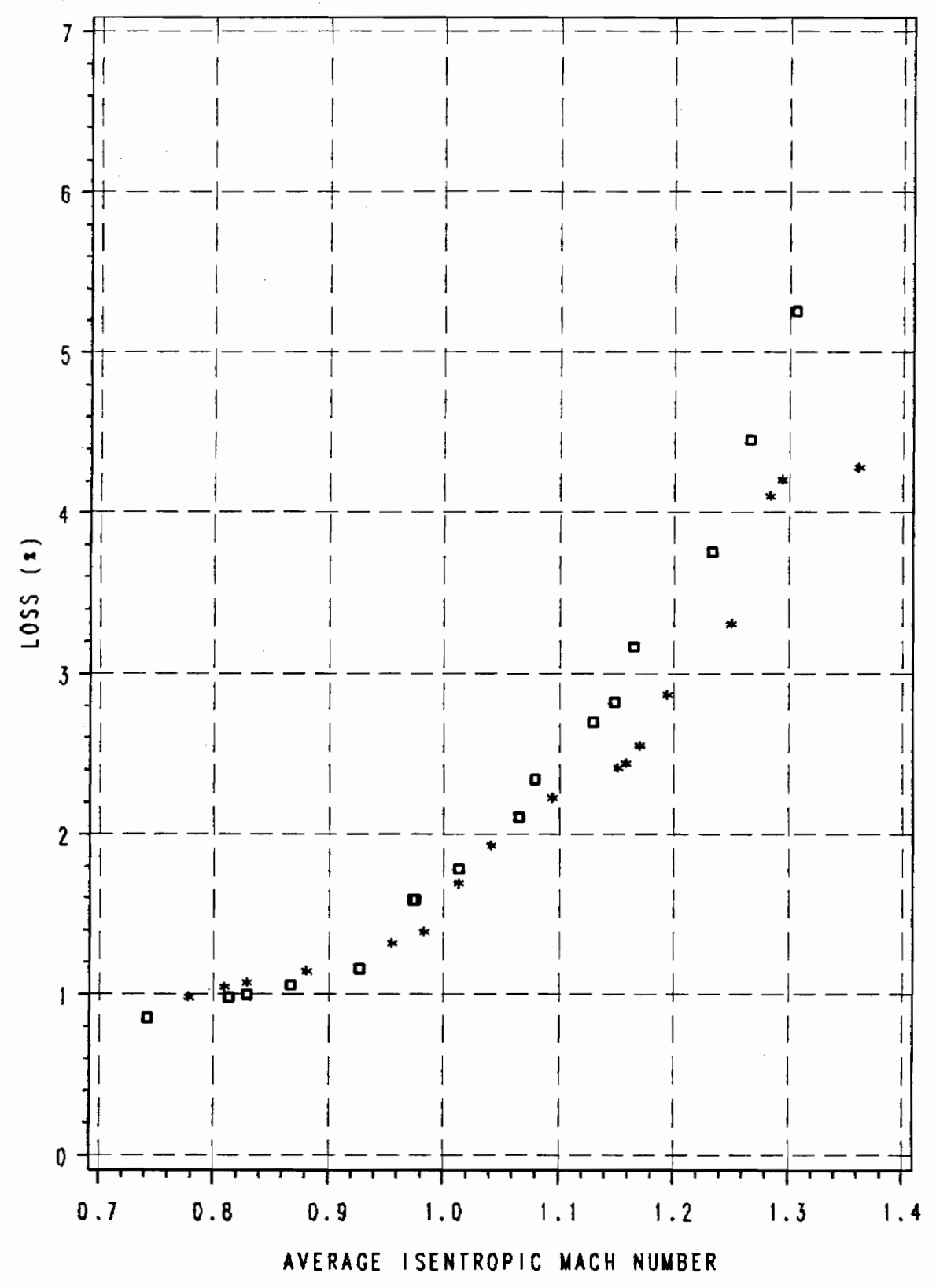

Figure 68. Corrected total pressure loss versus isentropic Mach number, Baseline cascade, Station 1:

Squares $=$ Apr90, Stars $=$ Dec 90 . 


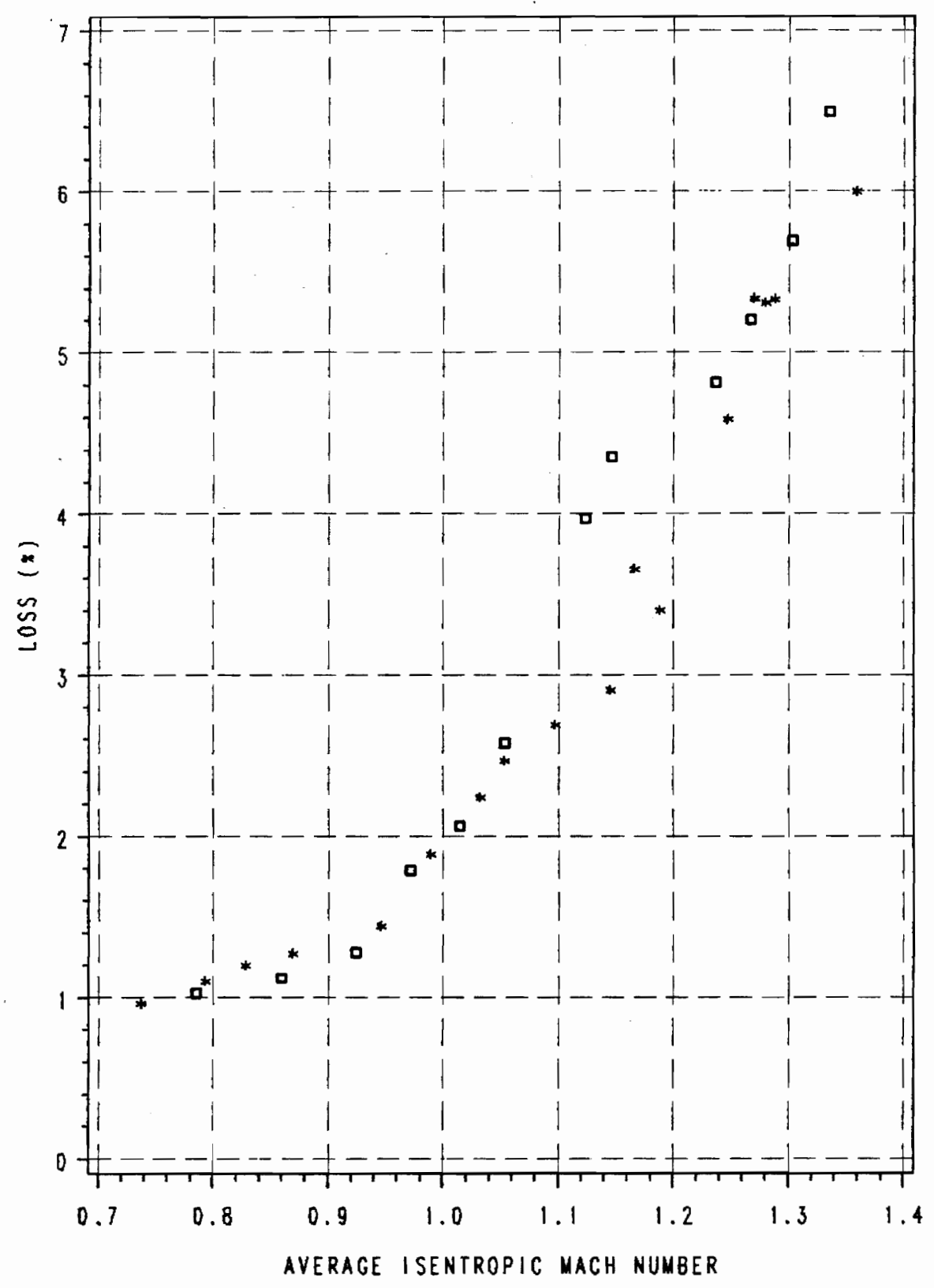

Figure 69. Corrected total pressure loss versus isentropic Mach number, Baseline cascade, Station 2:

Squares $=$ Apr90, Stars $=$ Dec90. 


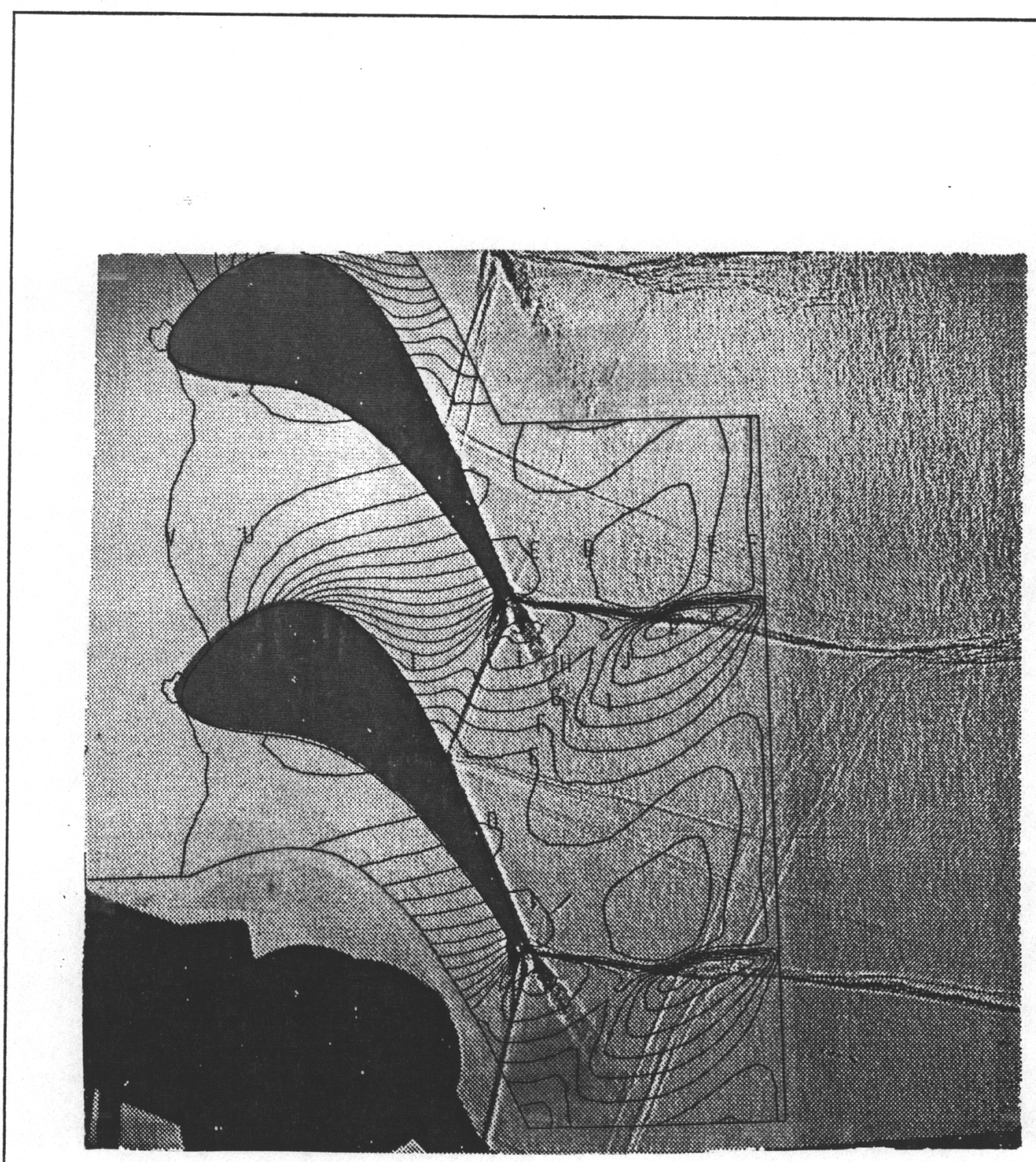

Figure 70. Overlay of calculated pressure contours and shadowgraph, UN10: Approximate isentropic Mach number: 1.30 


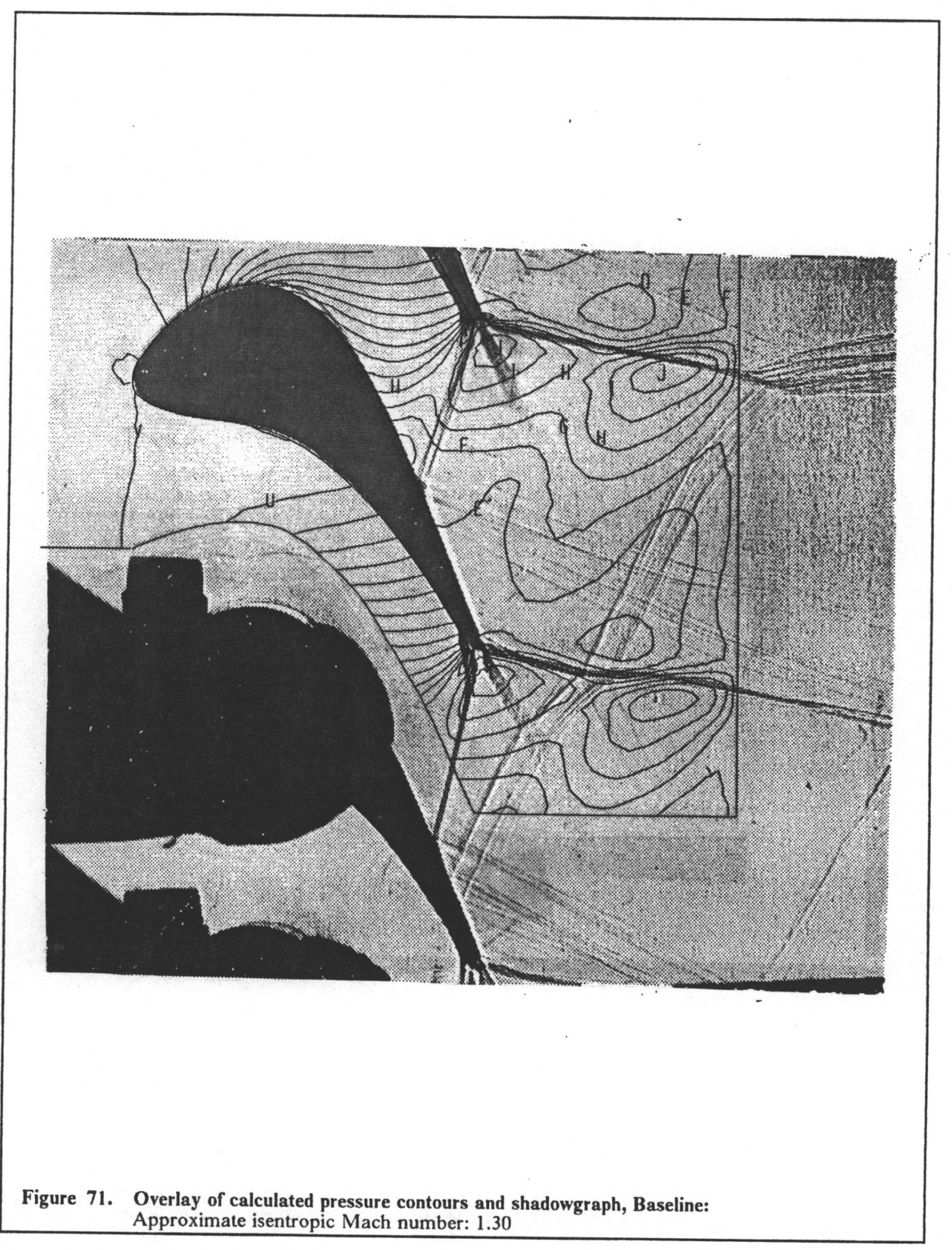




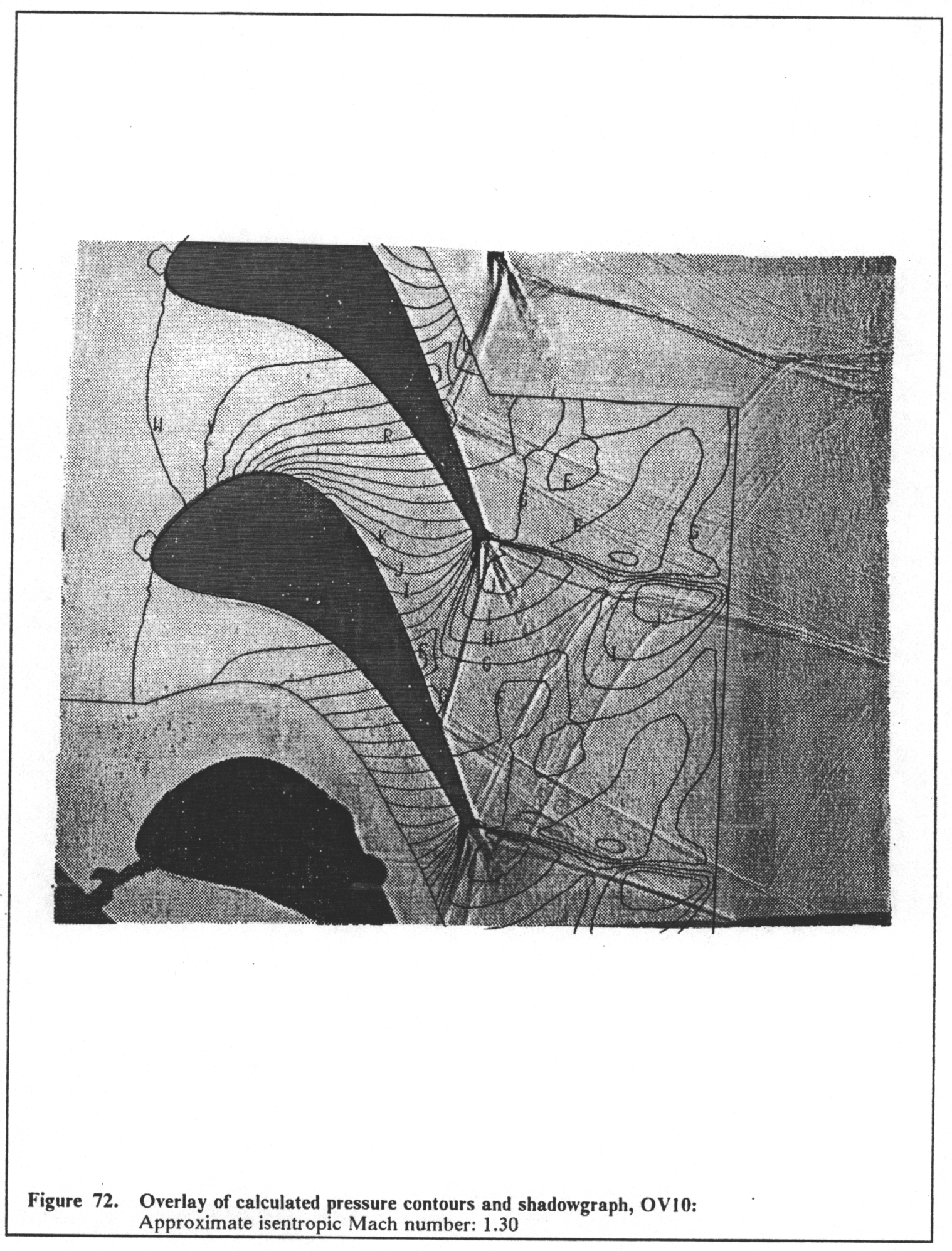




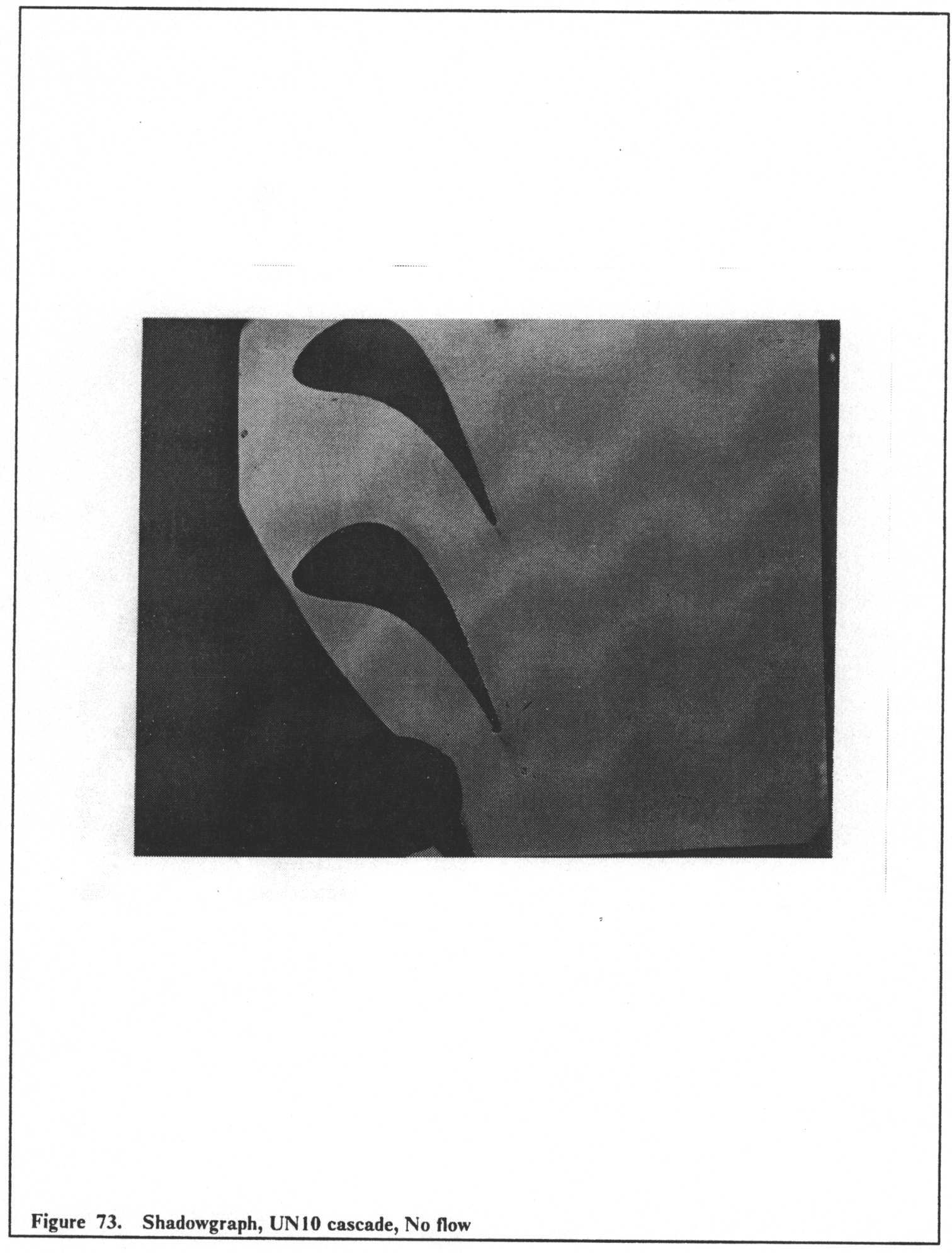





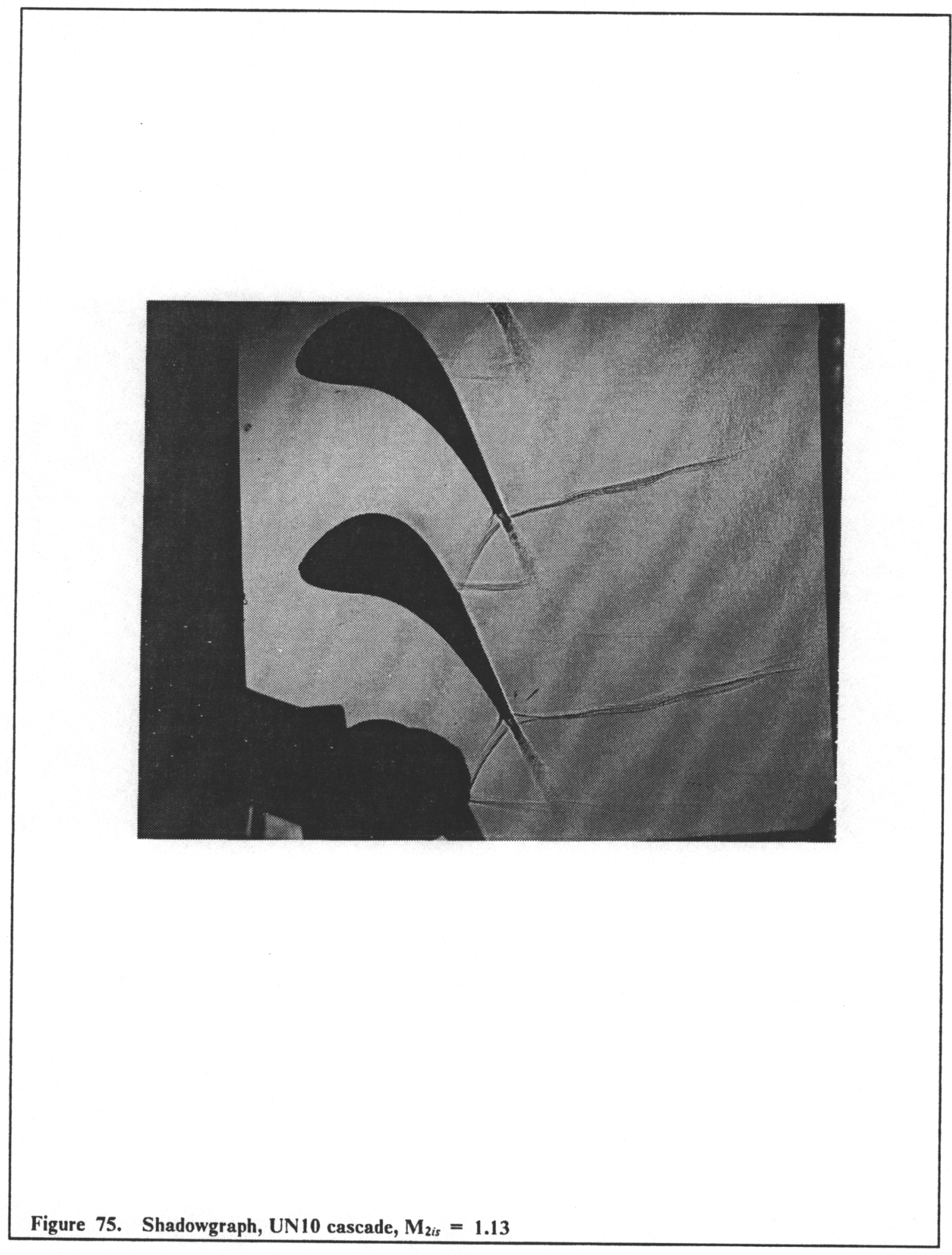




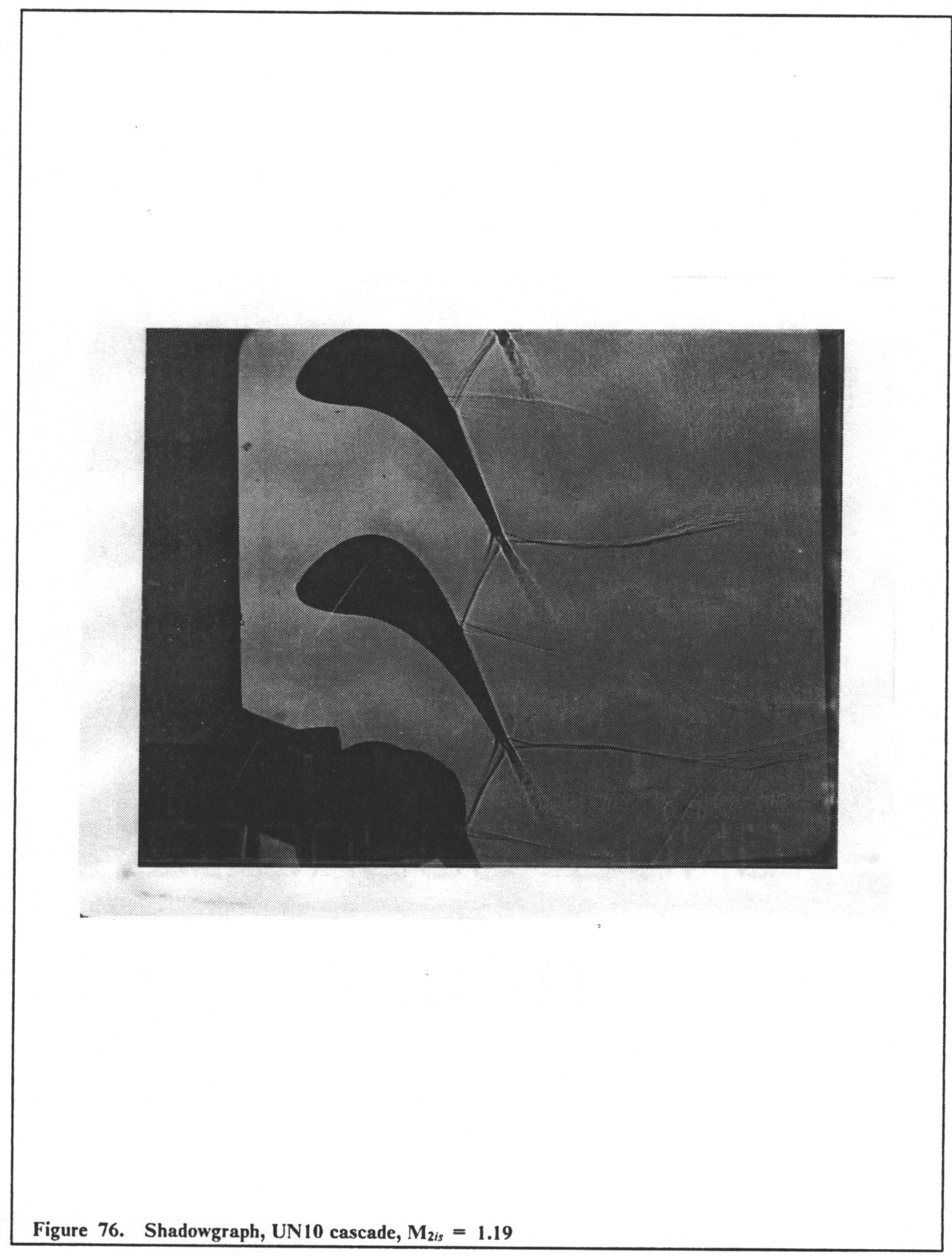




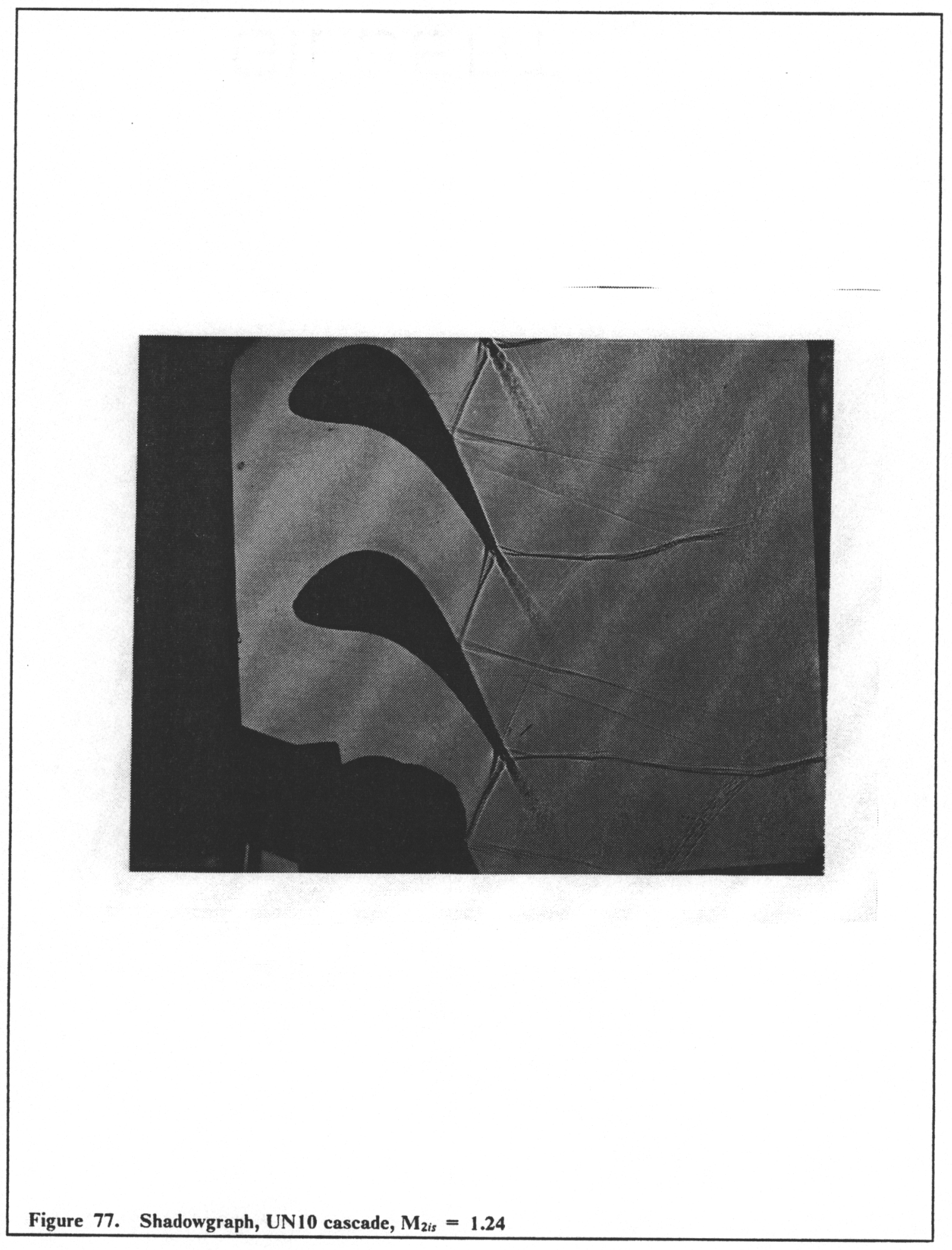




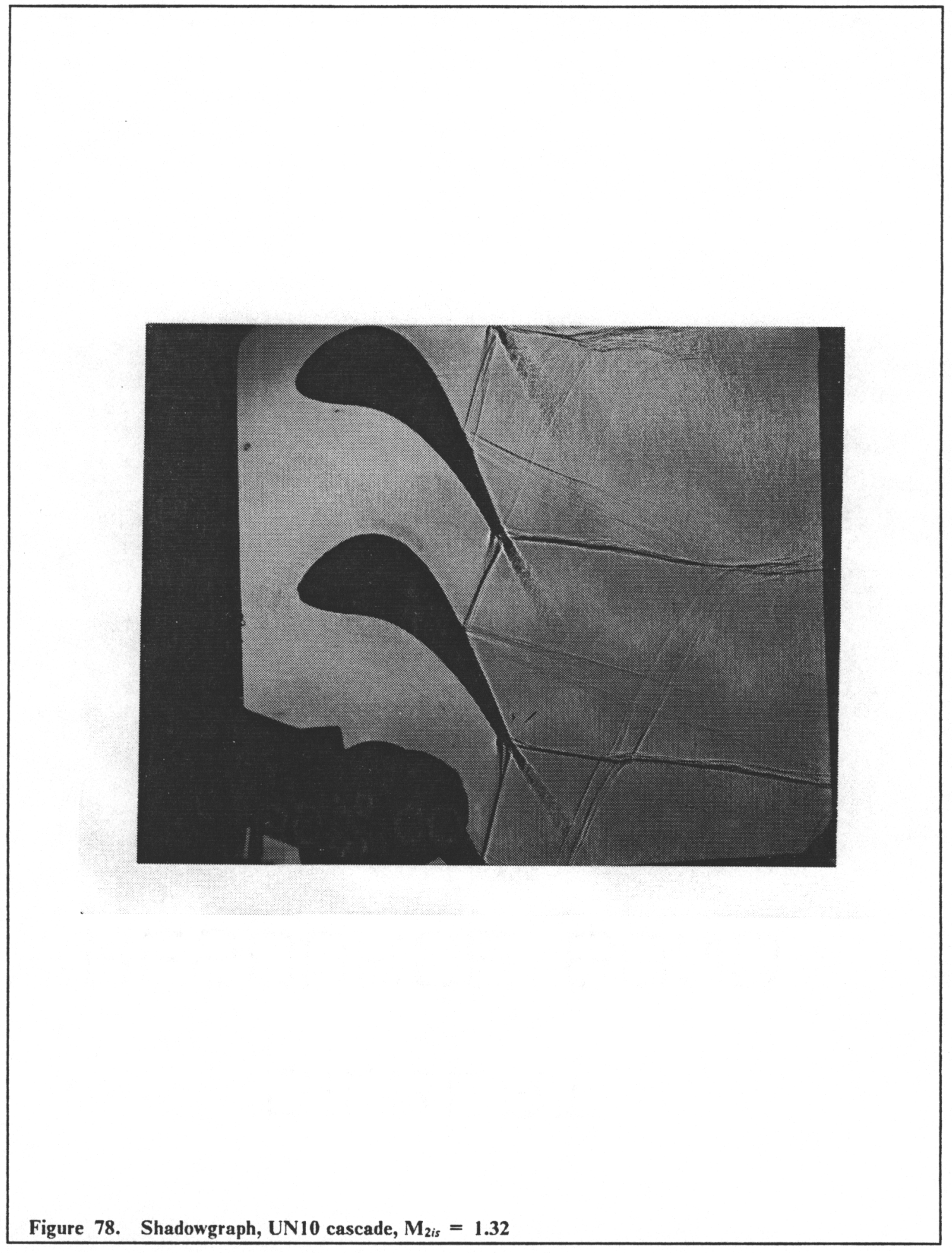




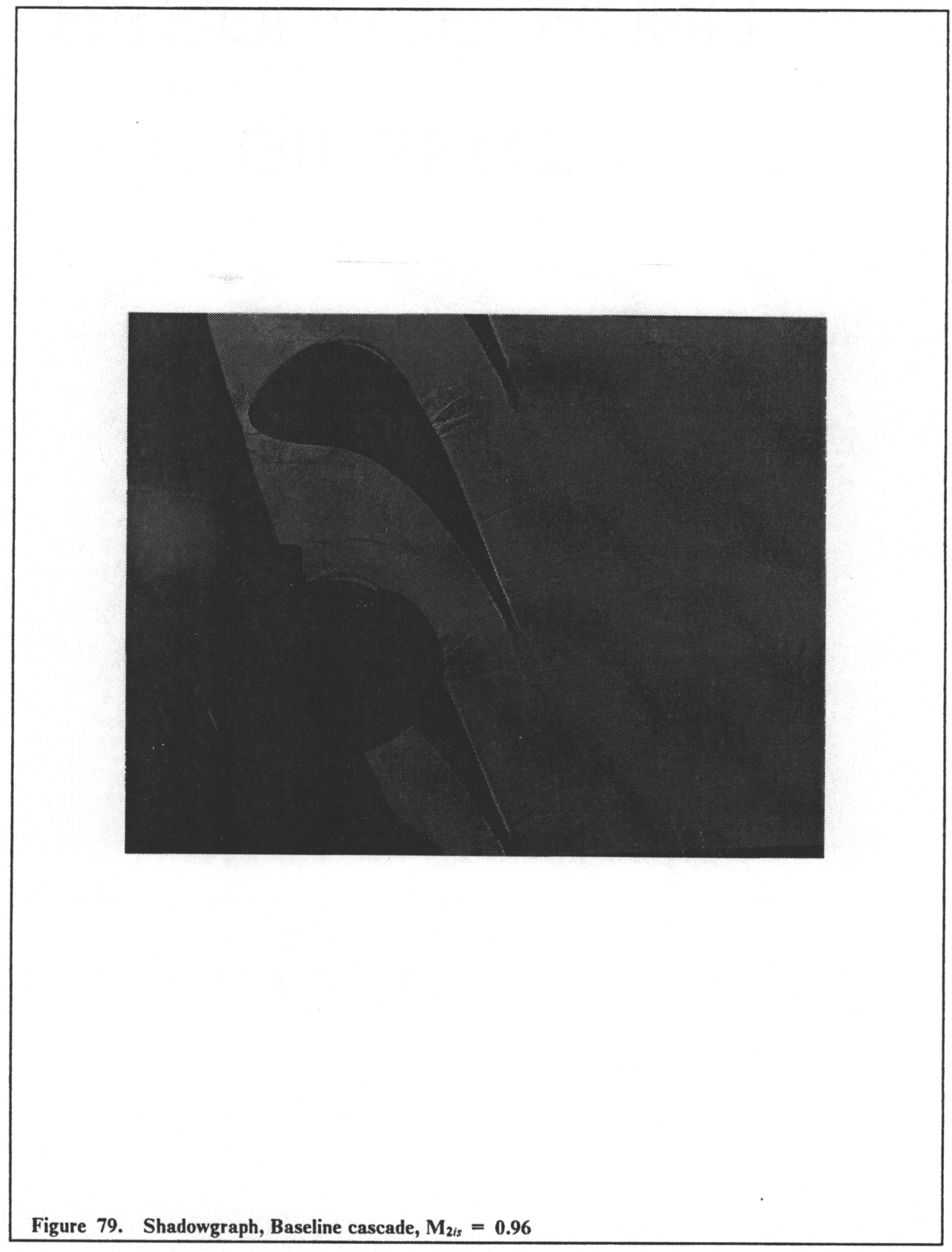




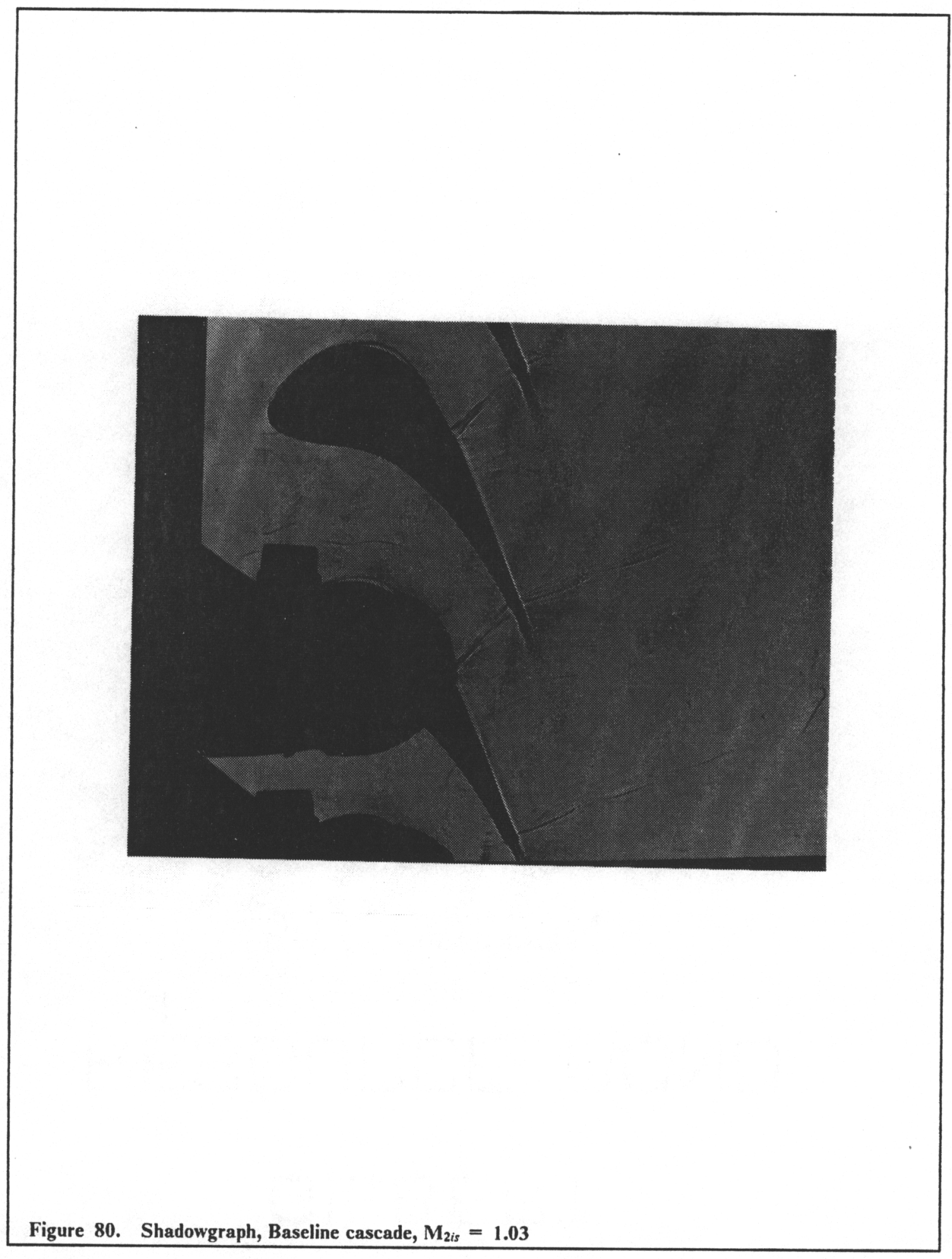




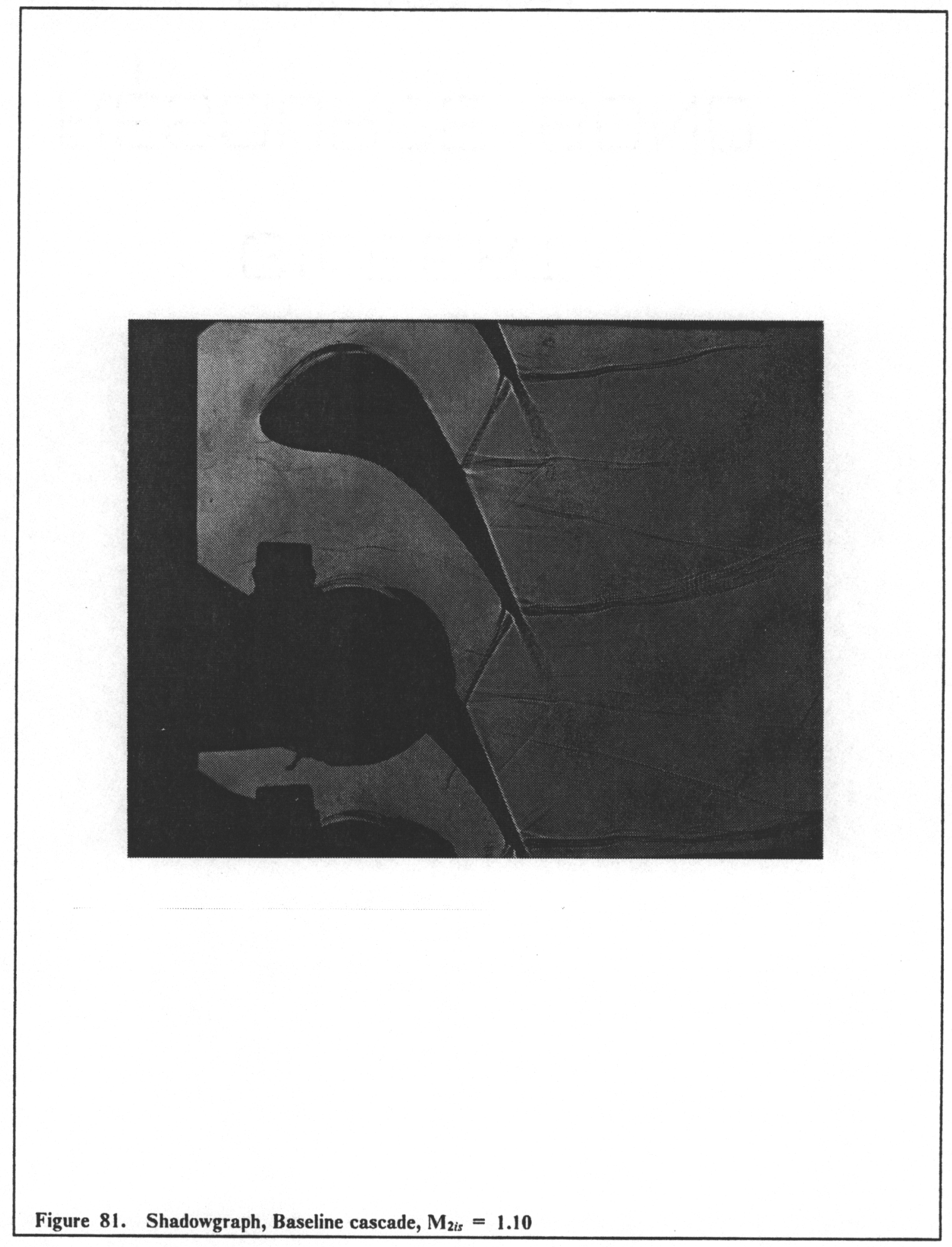




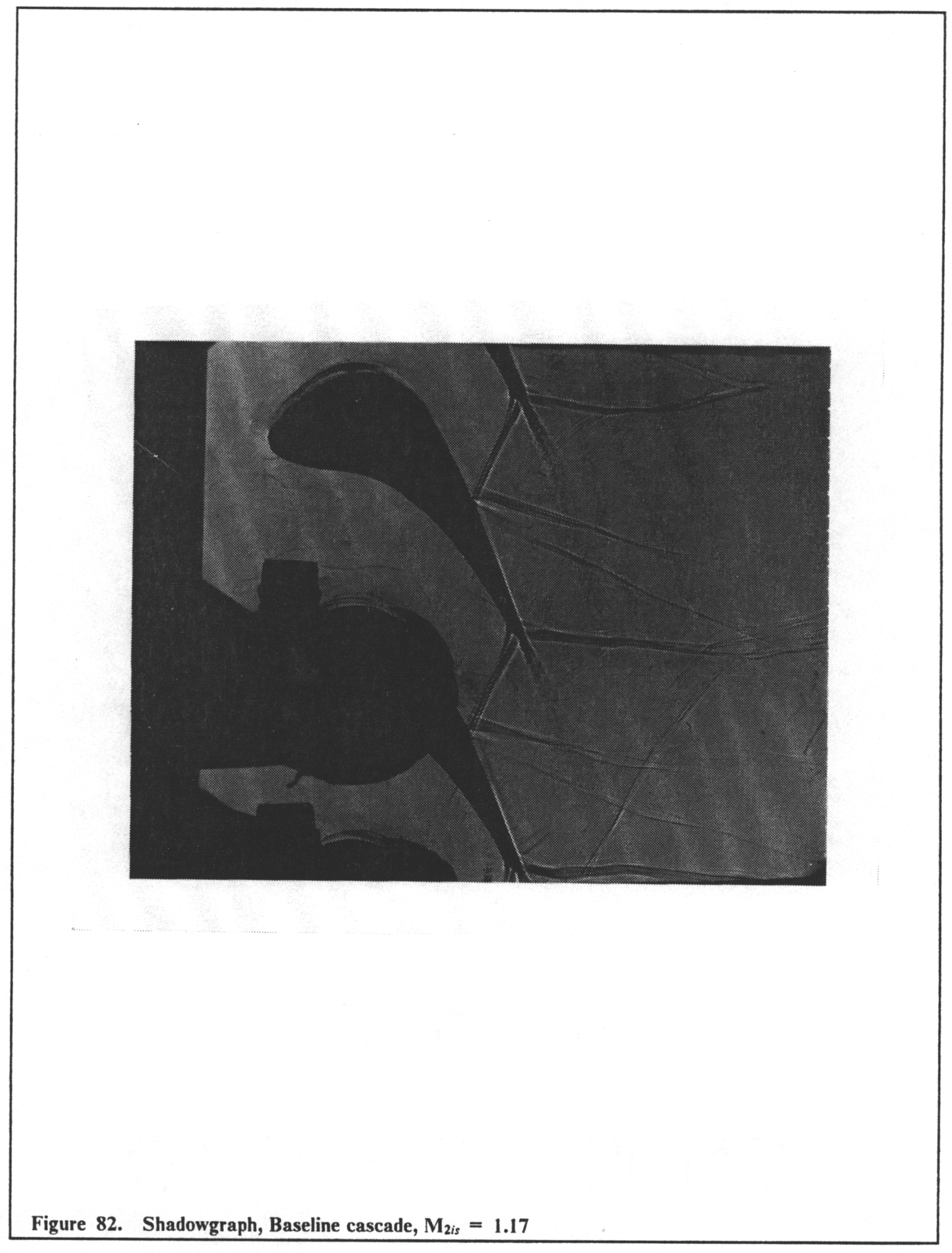




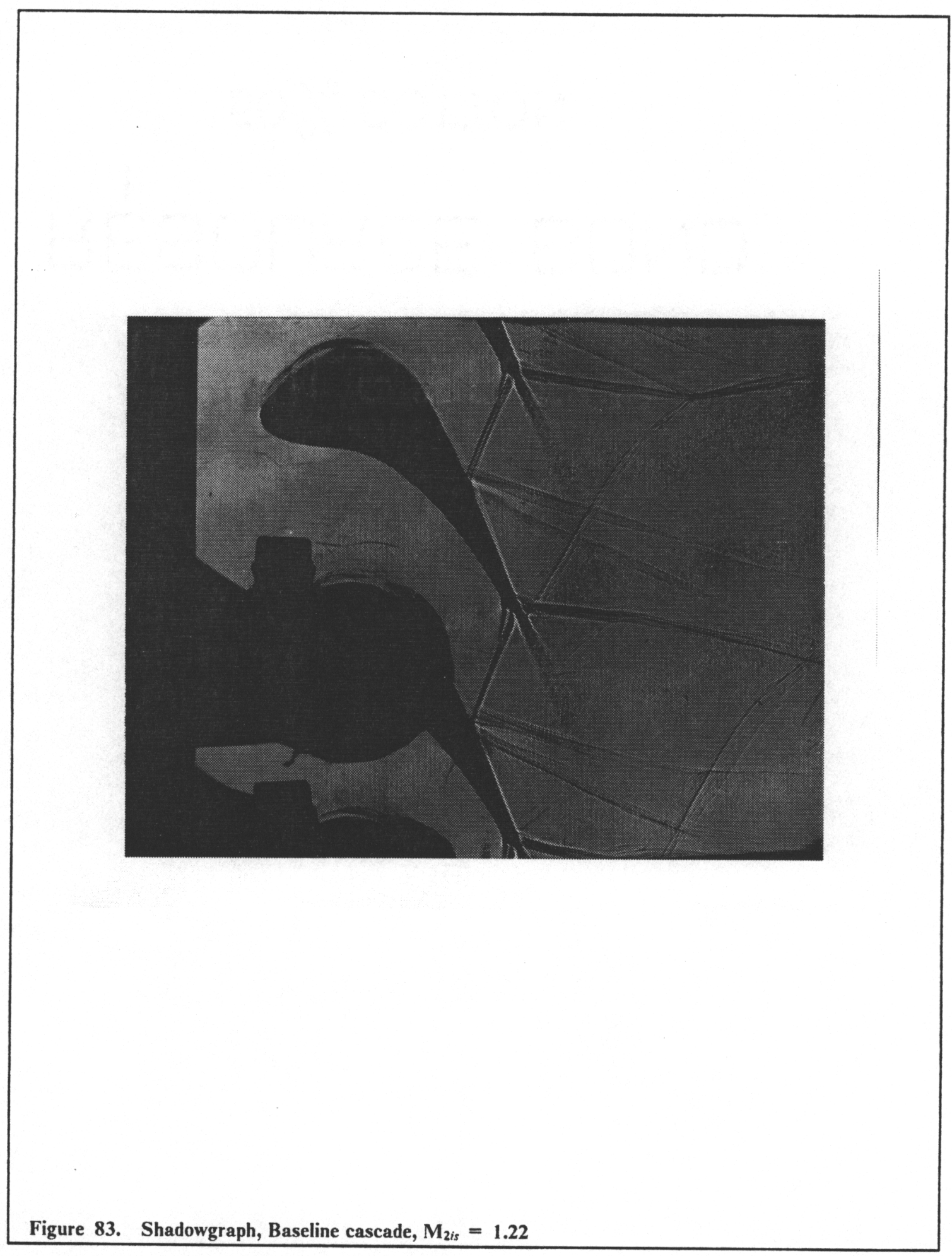




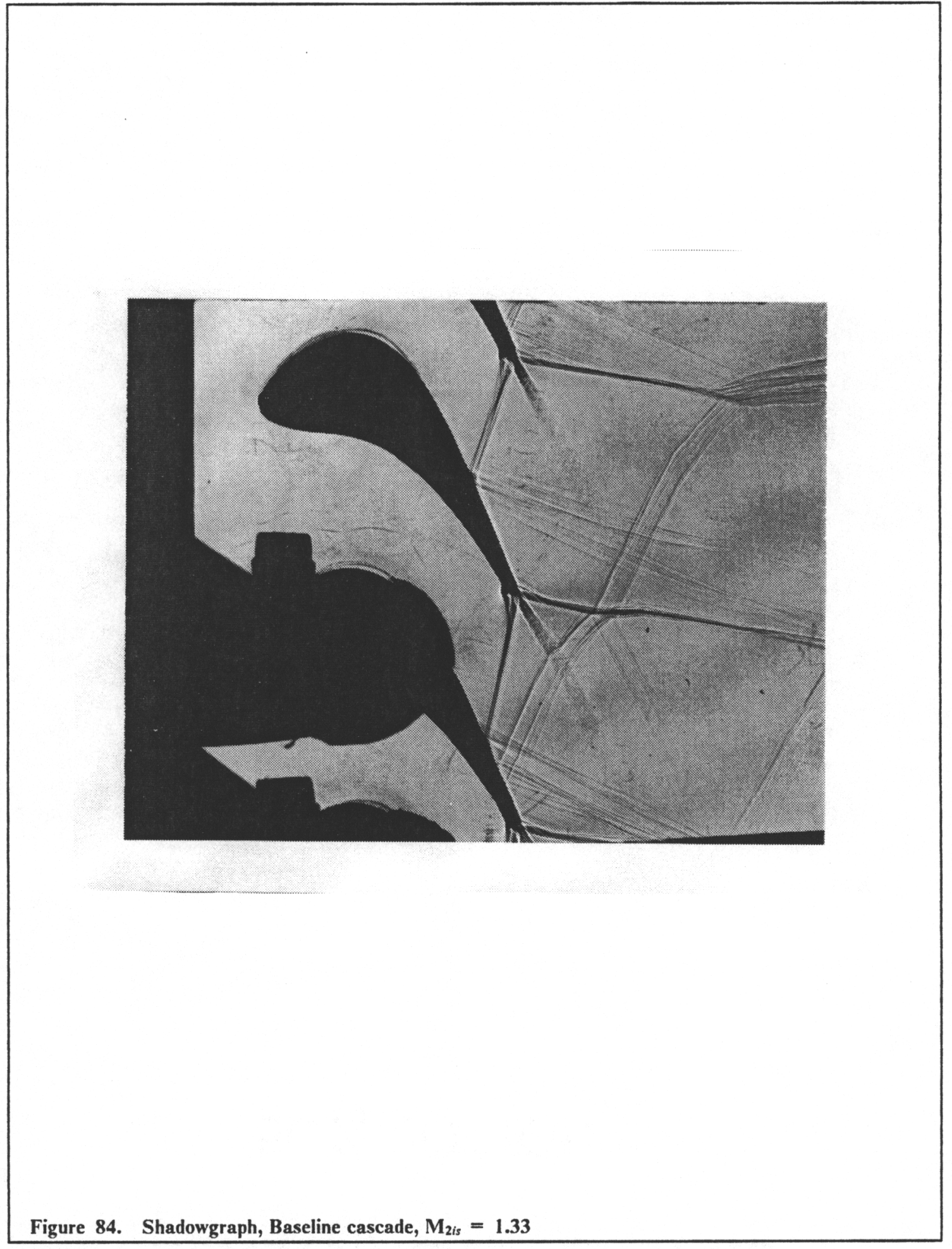




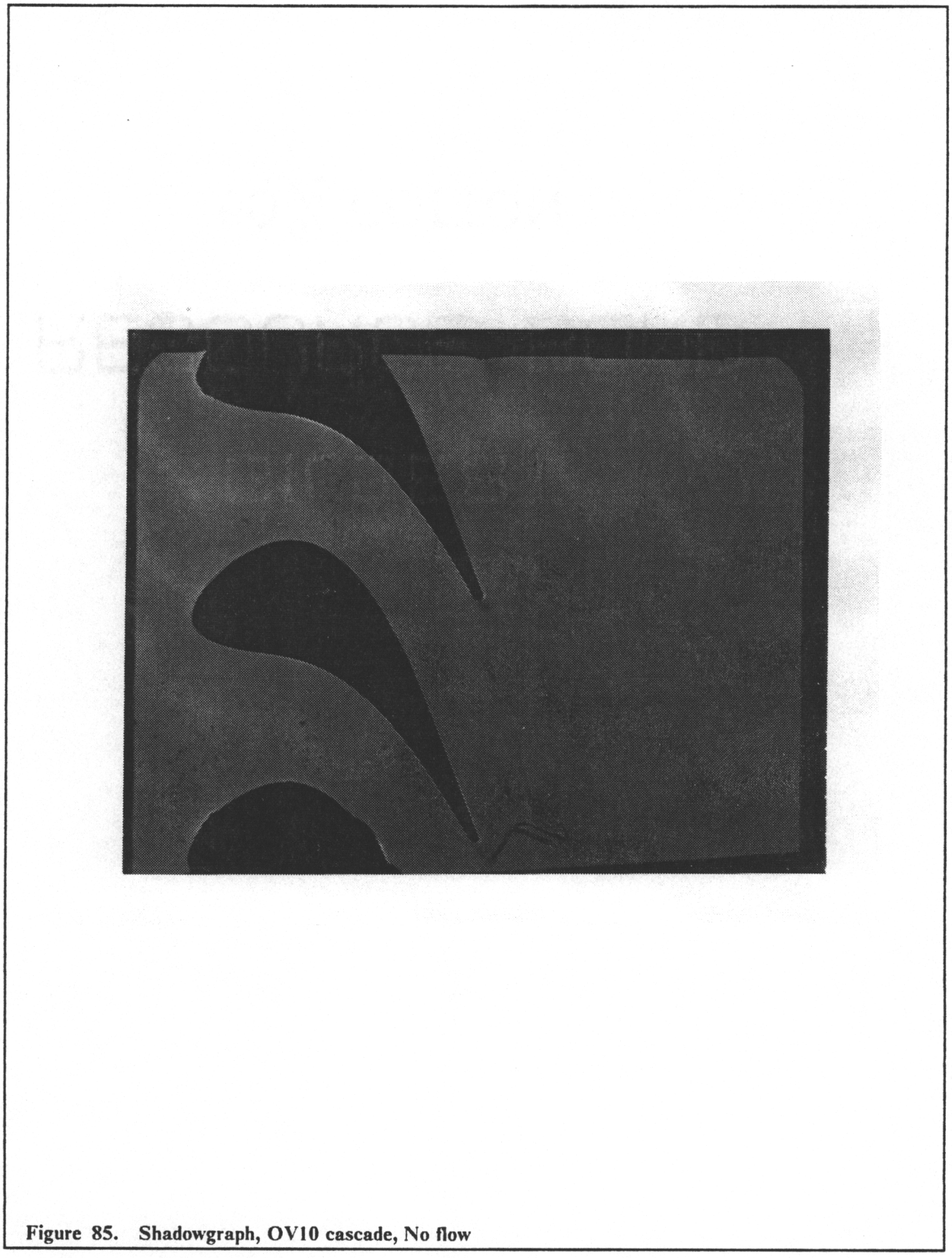




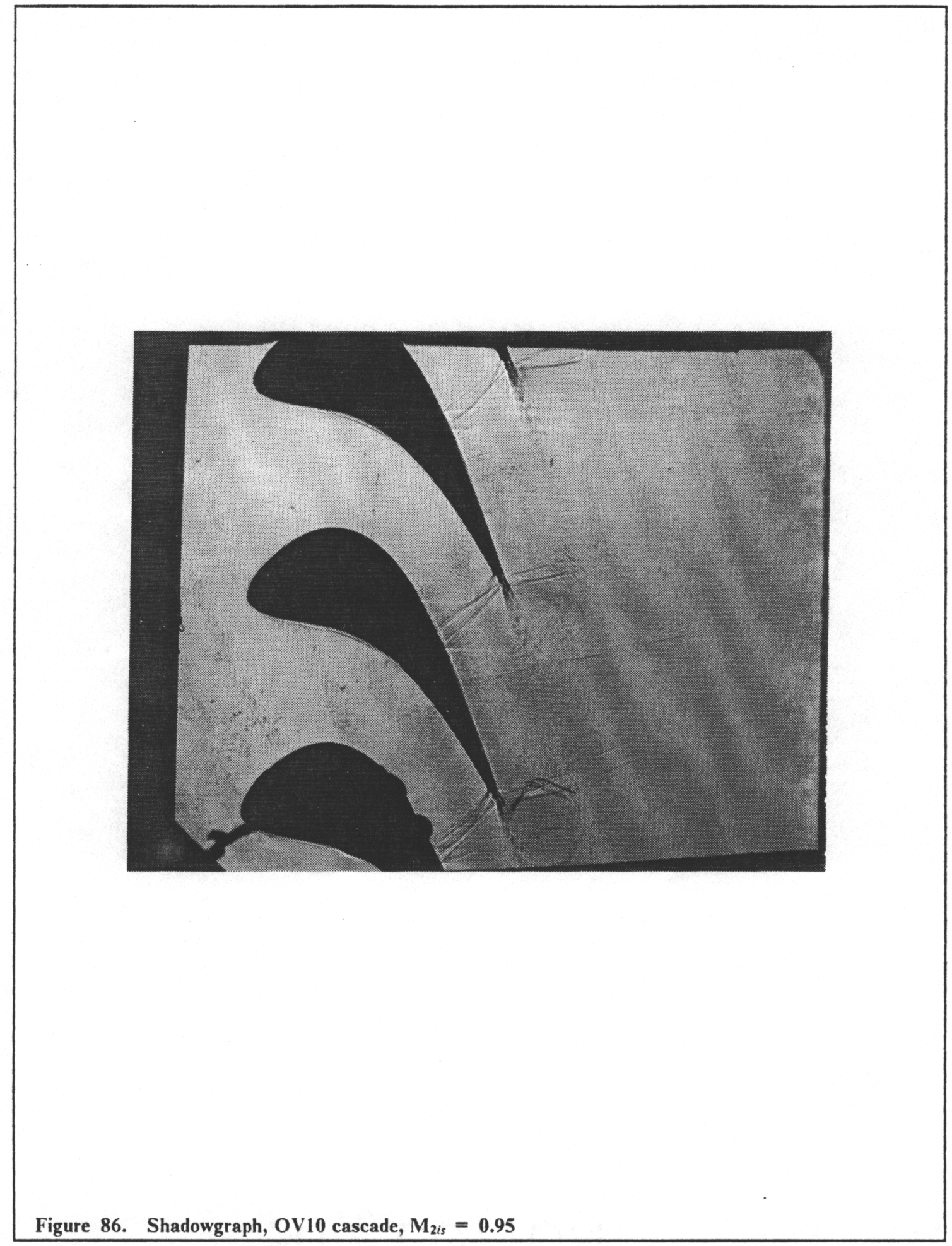




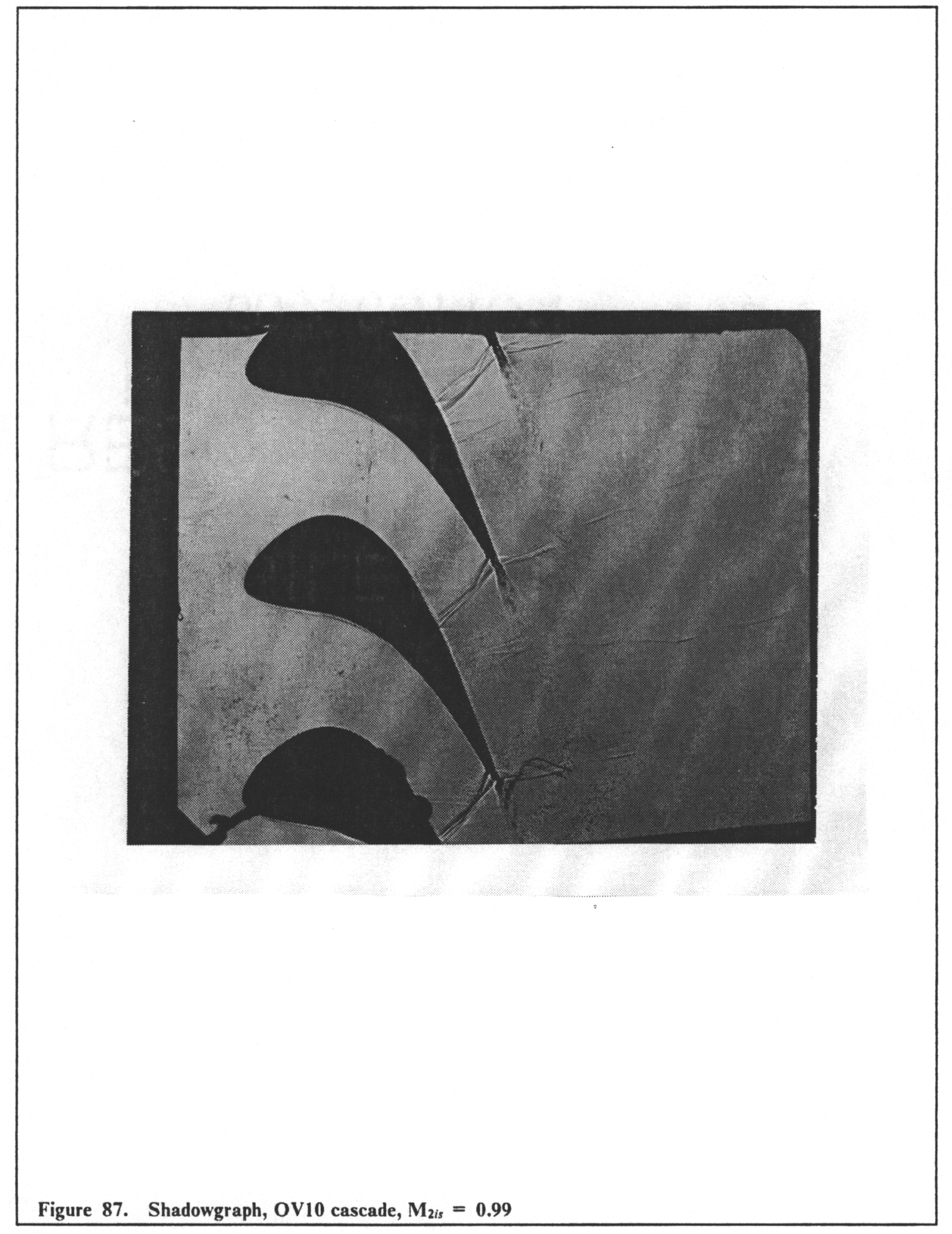




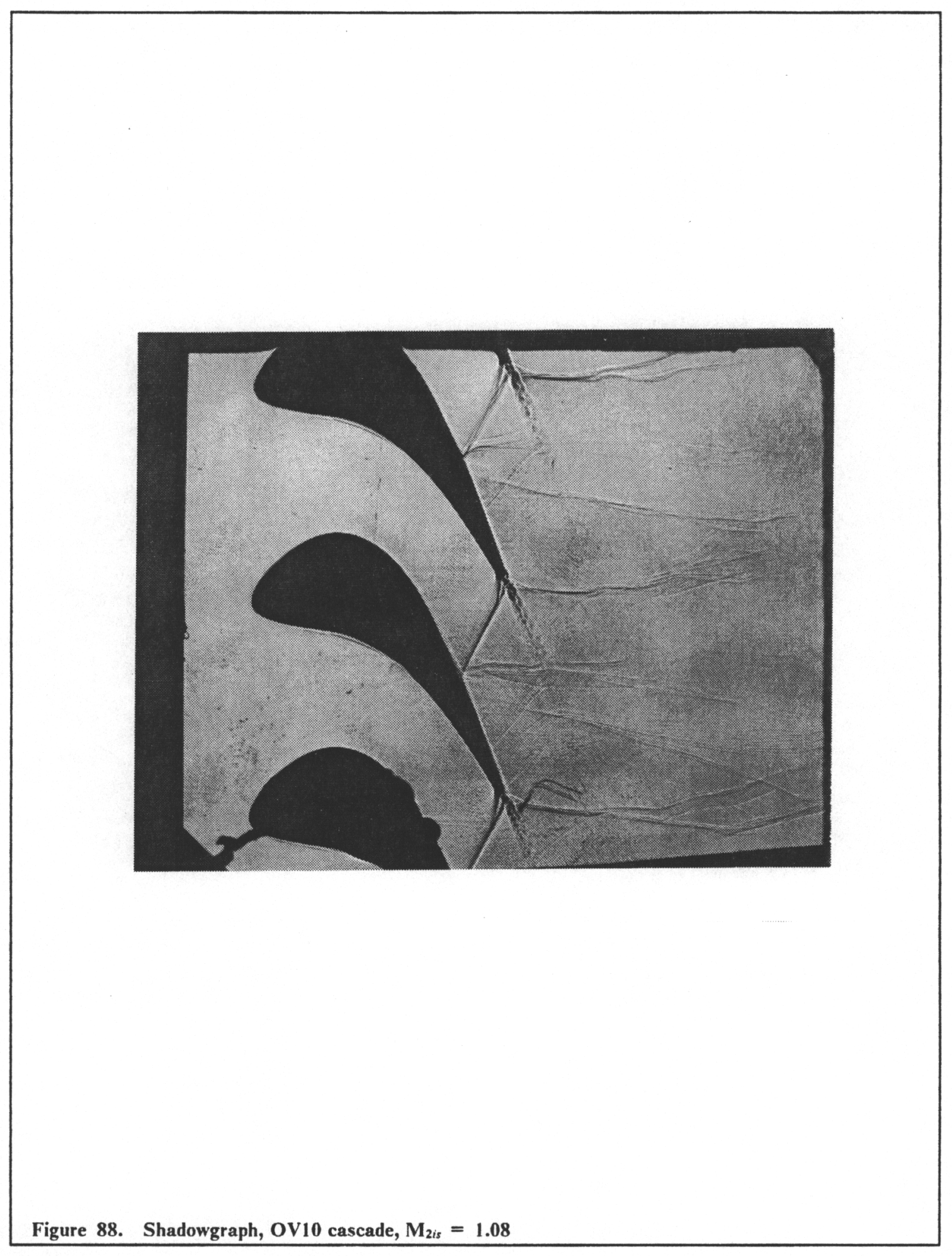




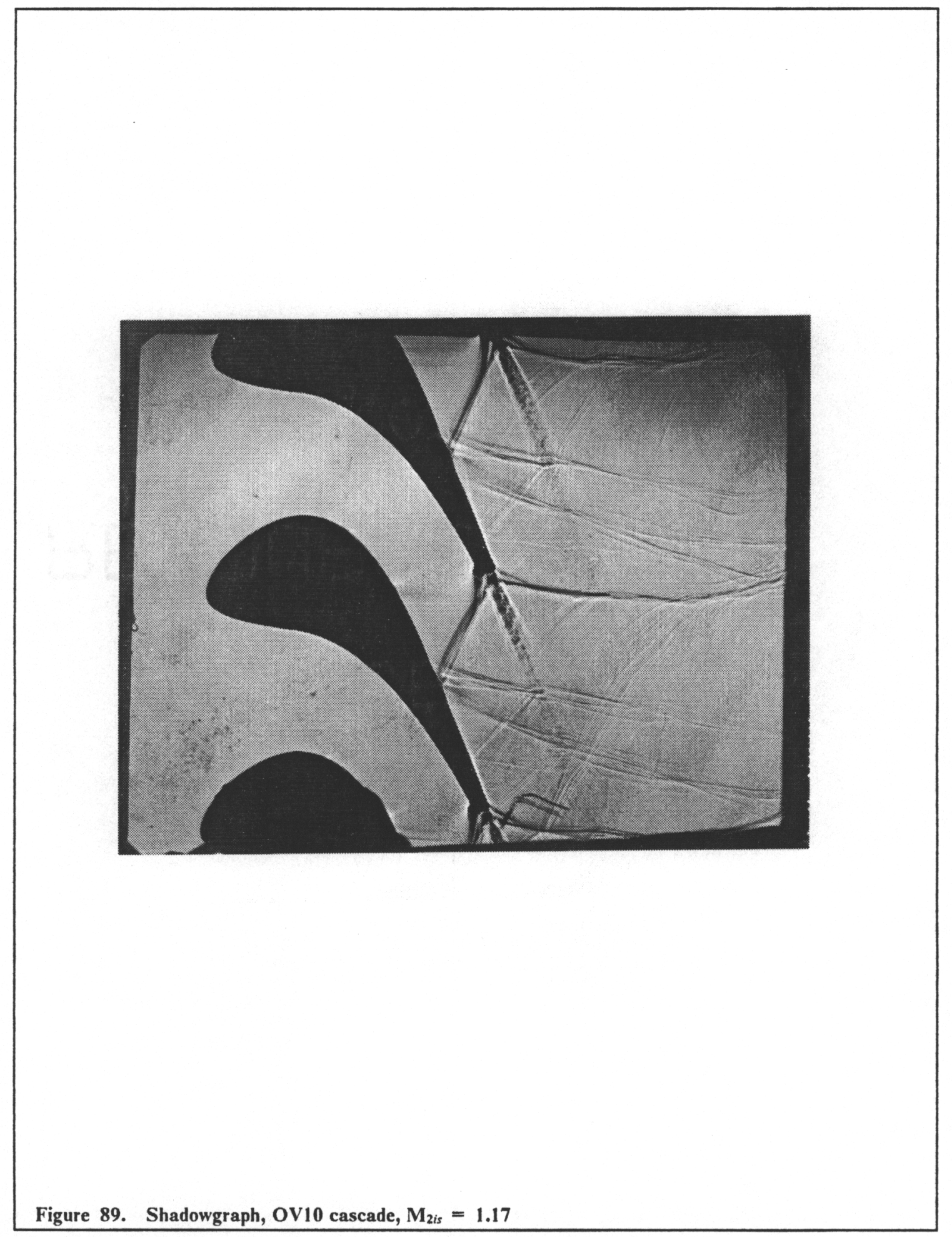




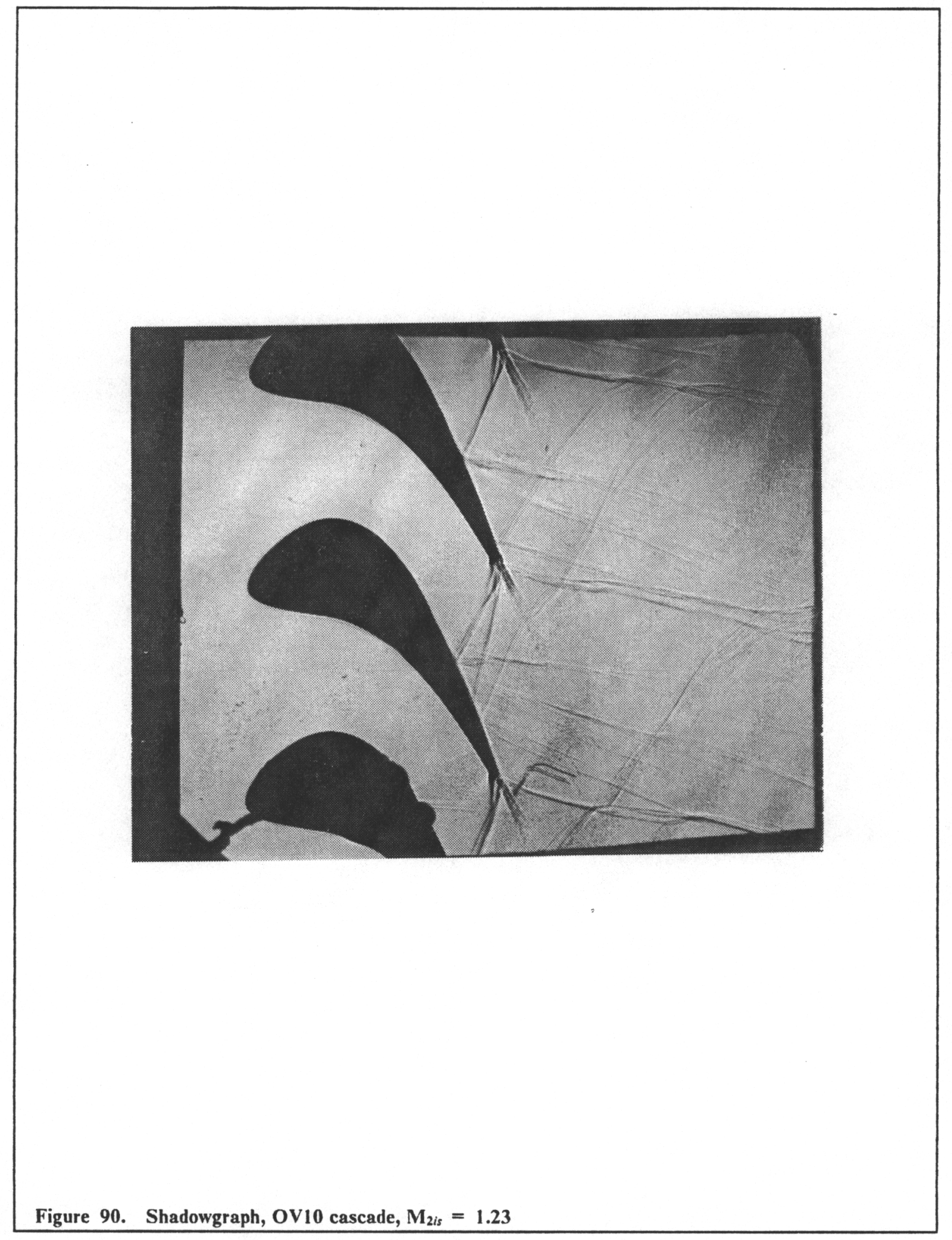




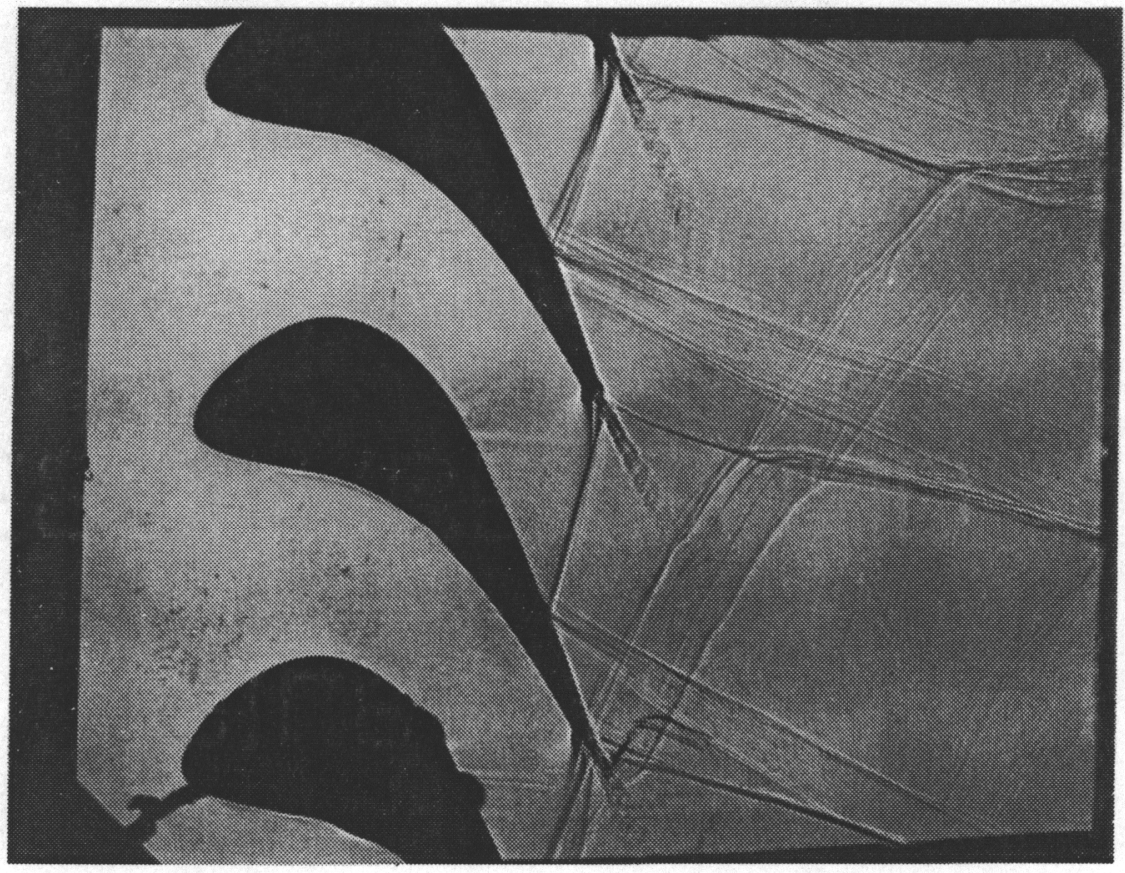

Figure 91. Shadowgraph, OV10 cascade, $M_{2 i s}=1.35$ 


\section{Vita}

The author was born on October 20, 1966 in Glassboro, NJ to Richard and Barbara Doughty. He graduated at the top of his class from Newark Senior High School in Newark, DE in May of 1985. He earned his Bachelor of Science Degree in Mechanical Engineering from Cornell University (Ithaca, NY) in May 1989, and then headed south to the warmer Virginia weather for graduate study. After one year at Virginia Tech he married Julie Ann Poole, culminating a four year long distance relationship. He is currently exploring possibilites for future graduate study or employment in the aircraft engine industry.

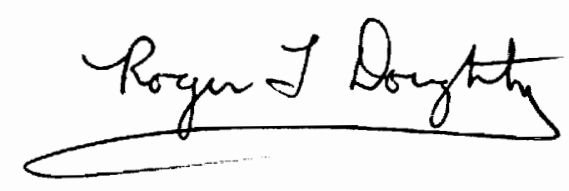

